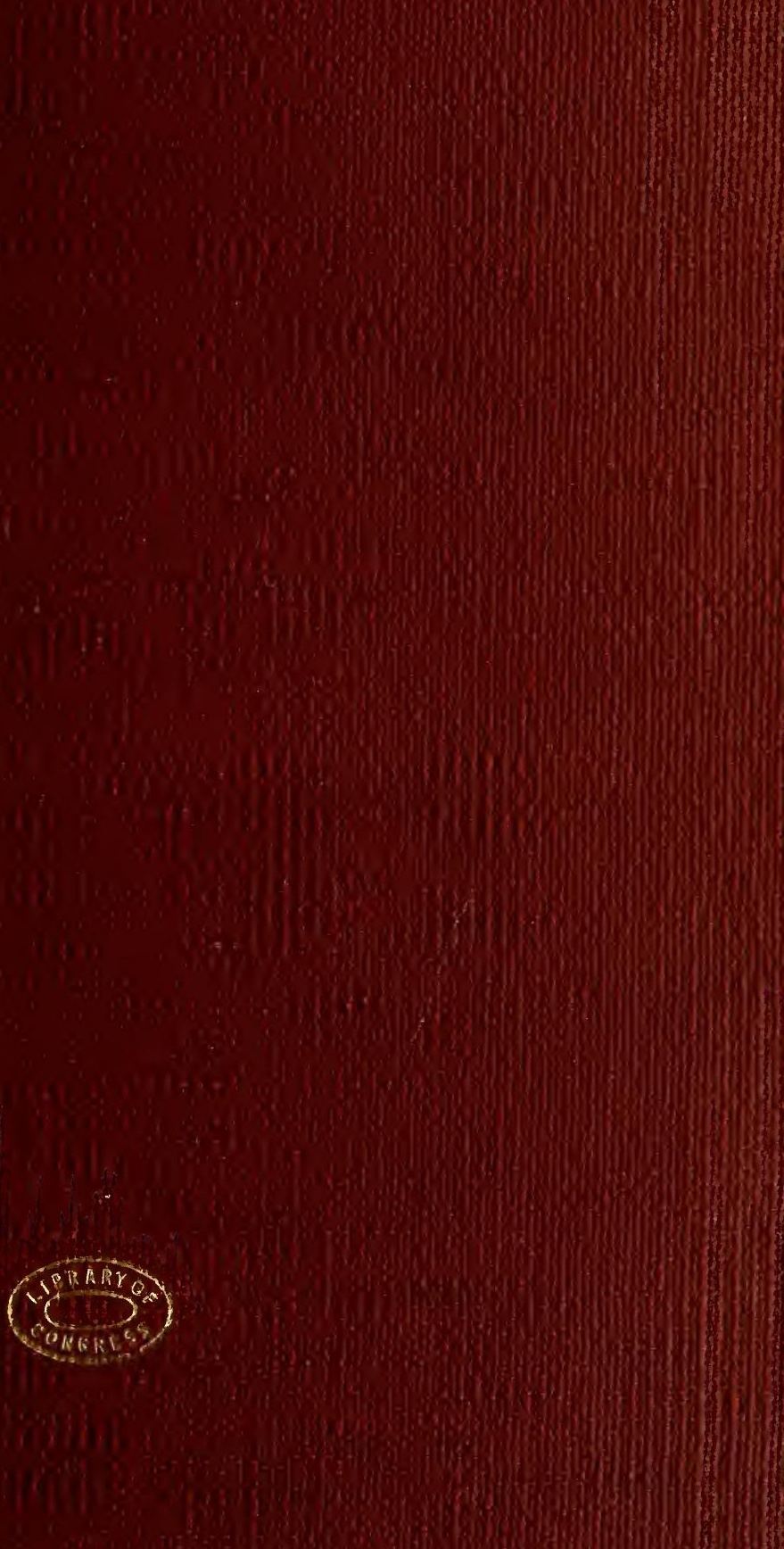




, 

THE

S I L K C UL'TURIST'S $\mathbb{M A N U A I :}$

OR

\section{A POPUIAR TREATISE}

ON THE PLANTING AND CULTIVATION OF MULBERRY TREES, THE REARING AND PROPAGATING OF SILK WORMS, AND THE PREPARATION OF THE RAW MATERIAL FOR EXPORTATION:

A D D RESE D T O THE

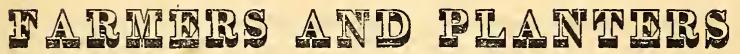
OF THE UNITED STATES:

\section{3 icter \\ B Y JOHN D'HOMERGUE.}

"A little worm-the source of countless riches, Take care of him, and he 'll take care of you."

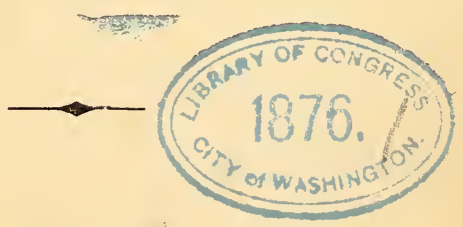

PHILADELPHIA.

PUBLISHED AND FOR SALE BT HOGAN \& THOMPSON, NO. 30 NORTH FOURTH STREET.

…....

1839

if 


\section{SFSH}

Entered according to Act of Congress, in the year 1839, by JOHN D'HOMERGUE,

in the Clerk's office of the District Court of the Eastern District of Pennsylvania.

J. THOMPSON, PRINTER. 


\section{DEDICATION.}

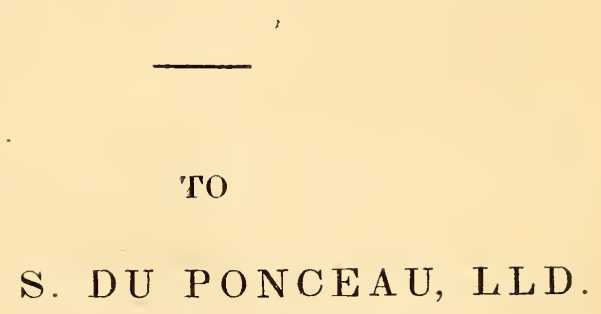

PETER S. DU PONCEAU, LLD.

PRESIDENT OF THE AMERICAN PHILOSOPHICAL SOCIETY

HELD AT PHILADELPHIA,

FOR PROMOTING USEFUL KNOWLEDGE, \&C. \&C.

\section{THIS WORK,}

IS RESPECTFULLY AND GRATEFULLY INSCRIBED BY

THE AUTHOR. 


\section{TABYE ON CONTENTS.}

The Author's Address to the Farmers and Planters of the United States, - - - - page ix.

Introduction,

$$
\begin{gathered}
\text { PAR T I. } \\
\text { Culture of the Mulbery Tree. }
\end{gathered}
$$

CHAPTER 1.

Of the different kinds of Mulberry trees, - - 7 White Mulberry, (Morus Alba, L.) - - $\quad 8$ Black Mulberry, (Morus Nigra,) - - $\quad$ - 10 Morus Broussonetica, (Mulberry of Constantinople,) 11 Morus Rubra, (Red Mulberry,) - - - $\quad$ - 11 Morus MTulicaulis, $\quad$ - $\quad$ - $\quad$ - $\quad$ - $\quad$ - 12 Dandolo or Moretti Mulberry, - $\quad$ - $\quad$ - 20 CHAPTER 2.

Peculiar substance in the Mulberry leaf, and the analogy of its constituent elements with silk, - 22

CHAPTER 3.

Of the Cultivation of Mulberry trees, $\quad-\quad-\quad-26$ This chapter embraces the following heads :-

1st. The manner of gathering and preserving the seed, - - - - - - - -

$2 \mathrm{~d}$. The soil proper for the raising and plantation of Mulberry trees, - $\quad$ - $\quad$ - $\quad$ - $\quad$ - 30 $3 \mathrm{~d}$. 'The various modes of propagating the Mulberry trees, - $\quad$ - $\quad$ - $\quad$ - $\quad$ - $31,35,38$ 4 th. The planting out of the young Mulberry trees, 42 5th. The grafting and budding Mulberry trees, 
6th. The culture, pruning, and management of the trees, - - - - - - - page 50

7th. The diseases of the Mulberry trees, - 53

P A R T I .

Of the Silk Worms.

CHAPTER 1.

Of the rearing of the Silk Worms; - - - 58

CHAPTER 2.

Method of Hatching the Silk Worms, - - -

CHAPTER 3.

Of the rearing of Silk Worms during their first ages, 66 CHAPTER 4.

Rearing of thể Silk. Worms in the fourth age, - $\quad 79$ CHAPTER 5.

Of the Fifth age-First period, - - - - $86^{\circ}$ CHA P'TER 6.

Second period of the Fifth age-the Spinning of the cocoons, - - - - - - - - 103 CHAPTER 7.

Of the gathering of the cocoons, and their management for sale or reproduction, - $\quad$ - 113 CHAPTER 8.

Of the changes of the Silk Worm, the coupling of the moth, and the gathering and preservation of the eggs,

$$
\text { CHAPTER } 9 .
$$

126

Remarks on different varieties of Silk Worms, Chapter 10.

Of the Diseases of Silk Worms,

CHA PTER 11.

Of Cocooneries, -

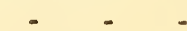

CHAPTER 12.

Extract from the work of Count Dandolo, on the subject of the preceding chapter, - 
Chapter 13.

Miscellaneous Observations, - - - - page 177

\section{P A R T I I I.}

Of the Preparation of Raw Silk for Exportation. CHAPTER 1.

Preliminary Observations, -

CHAPTER 2 .

Of the difficulty and importance of the art of Reeling, 214 CHAPTER 3.

Of the Filature system and its introduction into this country,

CHAPTER 4.

Of Filatures, their exterior form and internal arrange-

ments, and other matters relating to them, - 271 CHAPTER 5.

Of the Preparations for Reeling, - $\quad$ - $\quad$ - $\quad$ - 279

CHAPTER 6.

Of the different qualities of raw silk, _ - $\quad$ - 286

$$
\text { CHAPTER } 7 .
$$

Of the Reel and other apparatus, - - - - $\quad 294$

Explanation of the plate, figure 1, - $\quad$ - 302

Do do do $2, \quad$ - $\quad 307$

CHAPTER 8.

Of reeling and preparing the raw material for exportation,

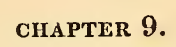

Of the progress of raw silk until it comes to the hands of the manufacturer, - $\quad-\quad-\quad-\quad-325$ CHAPTER 10.

Piedmontese Ordinance, concerning Filatures and silk reels, of the 8th of April, 1724, - $\quad$ - $\quad$ - 329

\section{A P P E N D I X.}

A. Mr. Boucher's Correspondence with Mr. Du Pon-

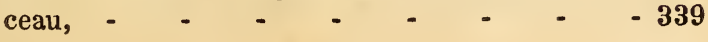


B. History of the Silk Bill, - - - - page 350

C. Additional Documents concerning the Silk Bill, - 365

D. Proceedings in the Legislature of Pennsylvania, on the presentation of a flag made of American Silk, bearing the colors of the United States, 401 
THE AUTHOR'S ADDRESS

TO THE

\section{FARMERS AND PLANTERS \\ OF 'THE UNITED STATES.}

\section{Gentlemen-}

Permit a stranger who has resided ten years in your country, who admires its liberal institutions and wholesome laws, and above all, the spirit of activity and enterprise by which your people is animated, which in the short period of fifty years, with the aid of intelligence and knowledge widely diffused, has raised these United States, from obscure colonies, to an eminent and highly dignified station among the nations of the earth, to address to you these fruits of his labor and experience in a branch of industry intimately connected with your agricultural pursuits; an industry peculiarly suited to your soil and to your climate, and which nature herself seems to point out to you as the source from which you are to derive immense riches.

Various attempts have been made from the 
first settlement of these colonies to introduce among you the culture of silk, all of which, for reasons which need not be stated here, have successively failed, and no trace of them remains, except a domestic manufacture of inferior sewing silk, which, during a period of near eighty years, has languished in one of your states, without producing the least benefit to the country at large, or even to the district of country in which it has been so long pursued, which has remained poor, in the midst of a rich produce, while at the foot of the Alps, that produce has been to the little Duchy of Piedmont, hardly equal in extent to some of your counties, and hardly visible on the map of Europe, the never failing source of immense wealth, so that the sovereign who rules over those mountains, by the title of king of Sardinia, from an insignificant island, which he also possesses, is numbered among the principal potentates of Europe.

Within the last ten or twelve years, this country has been awakened to this her most important interest. The national and state governments have done all in their power to give it their support. But their measures have been either vacillating, or lirected into wrong 
channels. They have hitherto had no success, and sometimes have produced effects different from those that were intended. At last the people have taken the matter into their own hands. Conventions have been called to discuss the best course to be pursued, and in expectation of something more effectual being done, mulberry trees have been imported and are now importing in large quantities, and command enormous prices; manuals and other works, for the instruction of the people in the cultivation of mulberry trees and the rearing of silk worms, are everywhere issuing from the press, so that, yielding to the general impulse, I venture to contribute my mite towards this important object.

The two first parts of this Treatise are exclusively devoted to the subjects above mentioned, to wit: the cultivation of mulberry trees and the rearing of silk worms. On these topics, you cannot expect much that is new. They have been exhausted by the European writers, and by your own; so that the multiplicity of books is become an inconvenience, from the difficulty of making a choice from among them. Some of them are filled with mi- 
first settlement of these colonies to introduce among you the culture of silk, all of which, for reasons which need not be stated here, have successively failed, and no trace of them remains, except a domestic manufacture of inferior sewing silk, which, during a period of near eighty years, has languished in one of your states, without producing the least benefit to the country at large, or even to the district of country in which it has been so long pursued, which has remained poor, in the midst of a rich produce, while at the foot of the Alps, that produce has been to the little Duchy of Piedmont, hardly equal in extent to some of your counties, and hardly visible on the map of Europe, the never failing source of immense wealth, so that the sovereign who rules over those mountains, by the title of king of Sardinia, from an insignificant island, which he also possesses, is numbered among the principal potentates of Europe.

Within the last ten or twelve years, this country has been awakened to this her most important interest. The national and state governments have done all in their power to give it their support. But their measures have been either vacillating, or directed into wrong 
channels. They have hitherto had no success, and sometimes have produced effects different from those that were intended. At last the people have taken the matter into their own hands. Conventions have been called to discuss the best course to be pursued, and in expectation of something more effectual being done, mulberry trees have been imported and are now importing in large quantities, and command enormous prices; manuals and other works, for the instruction of the people in the cultivation of mulberry trees and the rearing of silk worms, are everywhere issuing from the press, so that, yielding to the general impulse, I venture to contribute my mite towards this important object.

The two first parts of this Treatise are exclusively devoted to the subjects above mentioned, to wit: the cultivation of mulberry trees and the rearing of silk worms. On these topics, you cannot expect much that is new. They have been exhausted by the European writers, and by your own; so that the multiplicity of books is become an inconvenience, from the difficulty of making a choice from among them. Some of them are filled with mi- 
xii. THE AUTHOR'S ADDRESS.

nute details, of no real use in practice, others are too summary, and not a few are obscure and not easy to be understood. It is difficult for a writer to avoid every fault and to combine every advantage; I do not pretend to have done that. I have tried to say all that is necessary to be known and nothing more. I have aimed at a good method and arrangement of my subject, and above all, I have endeavored to express myself clearly, and to be understood by all. This is all the merit which a work of this kind can lay claim to, and I have done all in $\mathrm{my}$ power to entitle myself to it. I have made no display of useless learning; I have not wandered into the fields of history; my simple precepts are not accompanied by philosophical reflections; I have always kept in mind that I was speaking to practical farmers, and not to the members of an Historical or Philosophical Society. You will judge whether the end which I proposed to myself has been sufficiently attained.

The third part of this book relates to the art of reeling the silk from the cocoons, and pre. paring the rich material for exportation; for I must confess that I believe that this country is 
not yet ripe for the manufacture of silk. You have tried it in Connecticut in the humble manufacture of sewing silk. During eighty years you have made no progress in it, and your silk is yet unsaleable in our great markets. Eight years ago Congress laid a duty of 40 per cent. on foreign sewing silks, which if your domestic article had been merchantable, would have amounted to a prohibition; the consequence has been that foreign raw silk has been imported in large quantities from China and Bengal, and only twisted into sewing silk in your country. You purchase it every day as American silk; but you are deceived, it is not American. Congress, by passing this law, meant to encourage your agriculture, cousidering the making of sewing silk as connected with it, (which in fact it is not) and they have only encouraged the silk culturists of other countries, while your domestic industry has remained stationary; and has made no progress whatever. You will find all that fully explained in the third part of this work. In consequence of this duty of 40 per cent. on foreign sewing silk, interests have arisen in different parts of this country which may require to be attended to. It might perhaps be the cause 
xiv.

THE AUTHOR'S ADDRESS.

of much injury to those who have embarked in twisting establishments under it, if it were taken off too suddenly. This however is a matter which I have no doubt, will be properly considered.

The reason of this, gentlemen, (for I must speak candidly to you) is that you are not sufficiently skilled in the art of winding off the silk from the cocoons, and converting it into what is called raw sith. You have been told that it is a very easy thing, that every woman may do it with little or no instruction; some have said that a proper machine, (say the Piedmont reel) is all that is wanted, as if the machine could work without hands: but there are others, even men in high authority, who have gone farther and have told you that American ingenuity has improved on the Piedmont reel, and that a machine has been invented so perfect and so simple, that eren a child by means of it can without the least dificulty, reel silk admirably well. This, gentlemen, is an illusion greatly to be lamented. I shall be the last man to deny, or in the least to question the ingenuity of the countrymen of Franklin and of Fulton; but I have undertaken to prove, and I hope I have clearly 
proved, that what you are said to have performed, is beyond the power of man.

However extraordinary it may appear to you, it is not the less true, that the art of reeling silk from the cocoons is nice and difficult, that it lrequires instruction as well as experience, and that it is only to be learned by secing it well performed. It is not so with the rearing of silk worms; that is a science, which may be learned from books, but reeling is an art, which no books can teach. Franklin might become a natural philosopher, and Bowditch an astronomer, with only the aid of books and of native genius, but no bocks ever made a cabinet maker, a watch maker, or a jeweller, nor a skilful performer on a musical instrument. Arts are only to be taught by ocular and manual demonstration, and by practice for some time under the eye of a master. I have tried in the third part of this work to describe as well as I could, the whole process of the art of reeling; but, at the same time, I have acknowledged that all I have said is not sufficient to make you perfect in it, while, with the aid only of my two first books, you may learn to cultivate mulberry trees and to rear silk worms, in the most econo- 
xvi.

mical and most profitable, and consequently, in the most perfect manner.

I endeavor to prove in the third part of this book, and I hope I shall be able to convince you, that there is but one way of making the silk culture profitable to this country, and that is by adopting the European system of large filatures, which are establishments in which raw silk is prepared for exportation or manufacture, and that the making of sewing silk is no part of this business, but belongs to a separate profession, which is that of certain mechanics, called silk thronsters. Their business is to twist ran sith, as it comes from the reel into sewing silk, or into other forms, to be employed in different manufactures.

In those large filatures, young females are taught the art of reeling; some of them remain attached to the establishment, while others, after being sufficiently instructed, return to their homes, where they reel silk for themselves, and those are called domestic filatures.

By means of these establishments, the art of reeling silk in the perfection required for the European manufactures, is disseminated throughout the country, and raw silk, thus 
prepared, becomes a valuable article for sale at home, or exportation abroad.

But this is not all, these establishments afford regular and constant markets for cocoons, and encourage the farmers to produce them in great quantities, by attending to the rearing of silk worms; otherwise they will neglect this occupation, or their cocoons will lie useless upon their hands.

It may be said, and it has been said, that there is not yet a sufficiency of cocoons in this country, to begin with large filatures; but unless we so begin, there never will be, because there will not be sufficient encouragement to produce them. On the contrary, as soon as a filature is established, and a regular market opened, cocoons will appear in abundance, because it will be the interest of the farmers to produce them. When Genrgia was first settled, with only a handful of inhabitants, in the midst of a wilderness, the British began by establishing a filature at the town (then village, ) of Savannah; cocoons immediately appeared as it were by enchantment, and raw silk was sent to England in large quantities, considering the then situation of that infant colony, and so continued to 
be sent until the revolution, which put an end to that promising establishment. In the year 1769, (a few years before the Revolution,) your illustrious Franklin, who was then in England, but constantly watching over the interests of his beloved country, suggested to the American Philosophical Society, then lately instituted, and of which, though residing abroad, he had been elected the first president, the patriotic idea of introducing the culture of silk into Pennsylvania, and recommended beginning by establishing a filature. "That learned body took up the subject warmly, application was made to the Legislature, but, as it appears, without success; a private subscription was then recurred to, which had the desired effect.

Now mind, gentlemen, what the Society did on that occasion. They did not employ writers to compile manuals on the cultivation of mulberry trees, and the rearing of silk worms, much less on the various modes of manufacturing silk; they did not attempt to invent new machinery, nor offer premiums for improved reels; they did not give ear to the schemes of the quacks and pretenders of that day, but they determined at once upon the rational plan of a filature in this city. 
They knew that it would produce mulberry trees, and silk worms, and cocoons, and manuals in sufficient abundance.

The filature was established; the building was in Seventh street between Market and Arch streets, which was then out of the city: no trace of it now remains. I have been told that a skilful Frenchman was placed at the head of it; but the record from which these facts are taken, only says, that "the Society procured the necessary machine (no doubt the Piedmont reel,) to wind the cocoons." It adds that the silk reeled at that filature was "not inferior in goodness to the best from France or Italy." This sounds like exaggeration, even if Dandolo himself had been placed at the head of the filature. Be that as it may, the fact to which I wish to draw your attention is, that from the 25th of June to the 15th of August, 1771, (less than two months,) 2,300 pounds of cocoons were brought to the filature to be reeled, or were bought by the managers.*

Two thousand three hundred pounds of cocoons! where did these come from? Why, gentlemen, from Pennsylvania, New Jersey, and Delaware, not an ounce from any other place.

" Hazard's Register of Pennsylvania, vol. i., p. 64. 
This is almost miraculous; but it is not the less true, and shows what filatures can produce, and how superior this system is to any other that can be devised.

The revolution also put an end to this noble establishment.

I shall only add a recent example to complete this proof. When in 1830 and 1831, an experimental filature under my direction, was established at Philadelphia, cocoons were brought to us in abundance, from all parts of the Union, and they continued to come for a long time afterwards, (for the market had acquired a reputation) but then they could find no purchasers.

You see, gentlemen, that all that is required to insure the production of cocoons, is a good market, and that no such market can be established but by means of a filature.

I shall now proceed to speak to you of the last attempt which has been made to introduce the filature system into this country; but which has unfortunately failed. I shall be obliged to dwell at some length upon this subject.

In the year 1829, I published, in conjunction with Mr. Du Ponceau, a series of Essays, in which these principles were for the first 
time presented to the consideration of the American public. I had, then, just arrived from Marseilles, in France, where I made my residence, having abandoned the silk business, to which I had been brought up, and taken to other pursuits. I was induced to come to this country by the agent of a society established in this city for promoting the culture and manufacture of silk. I had no idea of these promoting societies. From the brilliant prospects held out to me by their agent, I saw the mines of Peru open before me; he showed me a papor written in an unknown congue, of which I could only make out the words " President," "Vice President," "Secretary," "Treasurer;" the word 'Treasurer struck me particularly. I expected no less than to be placed at the head of a large filature, to which, perhaps, a throwing establishment might be attached. On my arrival, instead of a company of capitalists, I found an association of about thirty excellent patriots, intent on promoting the culture, and even the manufacture of silk, but whose annual income did not exceed two or three hundred dollars. I was, therefore, completely disappointed. 
xxii. THE AUTHOR'S ADDRESS.

In this situation, it was my good fortune to become acquainted with John Vaughan, Esq., the stranger's friend, and one of the most active and efficient members of the American Philosophical Society. He introduced me to the President of that learned institution.

I had found great dificulty in making myself understood of my patriotic friends, (perhaps, because I did not speak their language,) I found none with that gentleman; he had never turned his attention before to the business of silk; but the explanations that I gave him snon made him familiar with it, at least, sufficiently to make him understand the difficulty and the importance of the art of reeling silk from the cocoons. He became convinced that the filature system was the only means by which the culture of silk could be made a source of profit to this country; he proposed to me to write a series of Essays to be published in the newspapers, in which the advantages of this system should be clearly explained to the American nation, and offered to give me his assistance.

The Essays were written, not without diffculty. Some errors crept in them, and it is 
astonishing there were not more, when it is considered that I did not know one word of the language, and that my kind assistant had not yet become sufficiently familiar with the details of this intricate subject. They were written in great haste, and successively appeared in the National Gazette, from which they were copied into almost all the newspapers of the union. This great popularity induced us to reprint them in a pamphlet, which went off rapidly. It attracted the attention of Congress, who referred it to their Committee on Agriculture, with power to enter fully upon the subject, and report on the best course to be pursued. The committee immediately perceived the importance, as well as the dificulty of the art of reeling, and how necessary it was that it should be brought to perfection in this country. They unanimously determined to establish a normal filature for the instruction of the people, and to place me at the head of it. By their Chairman, who was then the venerable Ambrose Spencer, of New York, they wrote to Mr. Du Ponceau, and desired him to ask of me on what terms I would accept that employment. They proposed a plan which Mr. Du Ponceau 
xxiv. THE AUTHOR'S ADDRESS.

thought could not becarried into execution without too much expense, and which was besides liable to many difficulties. He suggested another on a more moderate scale, to which I agreed, on the terms that he thought proper to dictate, and he was not disposed to throw away the money of the United States. This plan was adopted by the committee, and a bill, embracing all its details, was reported to Congress. It remained pending there during three sessions, and on the 22d of May, 1832, was finally rejected.

I have thought proper, gentlemen, to insert in the appendix to this work, a history of this bill, which has become generally known under the name of the silk bill, accompanied with authentic documents in confirmation of it. Several reasons have induced me to do this. In the first place, I believe that the silk bill will be considered as an important part of the history of silk in this country, and therefore, it seems necessary that it should be known with all the circumstances that attended it. But there is another reason which is personal to me, and which, in justice to myself, makes it necessary that it should be known in all its details. It has been reported in the 
newspapers and elsewhere, that I had applied to Congress for employment, and it has even been said in the debates upon the bill, that its only object was to set me up in my line of business. I owe to myself, not only to contradict these assertions, but to prove their falsity, in the clearest manner. I have a reputation to preserve as well as others, and I hope the time will come when it will be acknowledged that my feeble efforts to promote the true interest of this country, as far as they are connected with the silk culture, have not been entirely useless. It will appear from the documents that I publish, that I never made any application to Congress for aid or assistance of any kind, and that I only accepted the propositions that were made to me without any solicitation on my part.

I must own that the ill success of this bill astonished me greatly. I had every reason to believe that it would pass into a law, otherwise I would not have sacrificed three years of $\mathrm{my}$ life in the hope of its success. It was in fact offered to me. The unanimous report of the Committee on Agriculture and the favor with which it appeared to be received by Congress, completely deceived me. Other business had 
prevented its being acted upon during the two first sessions, but I had reason to believe that it was intended that it should pass. I would, however, have left this country, had it not been for the efforts that were made to induce me to remain; as you will see in the appendix, p. 362.

At the session of 1831 and 1832, a new Committee on Agriculture was appointed, of which the honorable Erastus Root of New York, was the chairman; that committee, I was informed, was opposed to the bill. Mr. Du Ponceau and I went to $W$ ashington, and appeared before

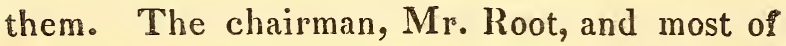
the members were decidedly hostile. We were examined, cross examined, and re-examined before that committee, and the result was that at least a majority of them changed their opinion entirely. The chairman, from an enemy, became a zealous friend to the bill, and supported it with eloquence in the debates upon it in Congress. The opinion of that body did not appear at that time to be so generally in favor of the measure as in the two preceding years. Some symptoms of hostility appeared, but still we expected that the friends to the bill would prevail. It had many powerful friends in and out of the House. 
The late President, General Jackson, was in favor of it. "I hope," said he to Mr. Du Ponceau, "that you will succeed." His name was made use of in Congress against the bill, on the last day of the discussion; but I am convinced that it was entirely without the knowledge or participation of the venerable chief.

The present Vice President, Richard $\mathbf{M}$. Johnson, was in favor of it, as may be seen by the paper in the appendix B, p. 362, to which his name is subscribed, with many others.

The then Secretary of State, Mr. Livingston, was in favor of it, and so was the honorable Andrew Stevenson, the Speaker of the House, although his situation obliged him to use more circumspection than he would otherwise have done.

Many others whom I might name were or expressed themselves of the same opinion. I do not speak of the eminent men who were amongst the respectable minority that voted for the bill, such as the venerable ex-President John Quincy Adams, Governor Everett of Massachusetts, Governor White of Louisiana, and other distinguished characters. In short, the bill had a host of friends at Washington, so that 
xxviii. THE AUTHOR'S ADDRESS.

I was truly astonished when I saw it rejected by a small majority the very day after its having reported to the House, by themselves sitting as a Committee of the Whole. To the last moment I thought that the result would be otherwise. Some of my too zealous friends have thought that Congress had done me wrong by coquetting with me during three sessions and at last suddenly letting me go. I am too well acquainted with the course of human affairs, to join in that opinion. The fact is, that Mr. Du Ponceau pressed Congress for a final decision at a time when the highest degree of agitation prevailed in it. There was the tariff, mullification, and the Bank of the United States, more than sufficient to distract the mind of many a wise politician. It is no wonder therefore, that in the midst of such a storm, my little bark was shipwrecked. I have no complaint to make of Congress, I ought to have known, that great political bodies are not always of the same mind; in future, I shall take care to follow the advice of the inspired writer: "Put not your trust in Princes!"

Some persons have thought that by endeavoring to introduce the filature system into this 
country, I was acting to the prejudice of France, which you know to be the greatest silk manufacturing country in the world. The late French Ambassador, Mr. Serurier, who resided at Washington while the silk bill was pending, was of that opinion, and expressed it publicly and without reserve. I am, gentlemen, a Frenchman, and nothing shall ever tempt me to betray the interest of my native Country. But I am of a different opinion, from that Minister. I was encouraged by the silk manufacturers of France, who are the most interested in the question. France wants raw silk, and I thought, and still think, that she may as well purchase it from the United States, as from Italy and other countries for her manufactures; as the time is far distant when America will rival her admirable tissues, which no other nation has yet succeeded to imitate. With the utmost respect for Mr. Serurier's opinion, I think he was, in this case, under a wrong impression, in which I cannot participate.

But whether he was right or wrong: I have reason to be astonished that his opposition, which was well known at Washington, in and out of Congress, did not ensure the passage of the silk bill, for it showed that he well un-

$$
\text { c* }
$$


derstood that the plan I proposed, if adopted by the nation, could not fail to succeed. I am, therefore, right in ascribing its failure to the agitated state of the public mind at that time. A bill, the mere proposal of which, excited the jealousy of foreign nations, would in other times, have passed unanimously. But fate had decided it otherwise.

After the lapse of six years, I have been much gratified to find that when the silk bill, as I thought, was almost forgotten, a third committee on agriculture, at the head of which is the honorable J.F. Randolph, of New Jersey, expressed their approbation of its principles, as suited to the time when it was proposed, though it was at present useless, for a reason which you will see explained in the following extracts from their report of the 20th of April, 1838. They express themselves in these words :

"Thus perished the first important measure proposed by the nation to promote the production of silk in this country; a measure which the committee believe, with the lights then in existence, was wise, prudent, and im. portant, but which the subsequent ingenuity and experience of our countrymen now render 
unnecessary; believing; as they do, that the recent improvements in reeling will do more in a few weeks than the establishment of many normal schools upon the old plan would do in many years."-(Report, p. 6.)

In another part of the same report it is said, that, "the dull, tedious method of reeling by hand, which required a regular apprenticeship to learn, and years to acquire facility in the use of, has given way to the new patent reel, by which a person (even a child) may learn in a few hours to reel, with great ease and expedition, a much more even thread than by the old process." - (p. 5.)

Then, gentlemen, the whole of this great question is reduced to a single point, which is a matter of fact, which any mechanician, with the light that this work throws upon the subject, may in a moment decide. If your country is really possessed of that wonderful machine, that Aladdin's lamp so loudly proclaimed, then all I have written must go for nothing, and I shall rejoice with you at the prospect of immense wealth that it opens to view; if on the contrary it should be found, as I have attempted to demonstrate, that no machinery whatever 
can produce the effects that are pretended; then the system which I recommend, that is the filature system, which was the basis of the silk bill, must be now as wise, as prudent and as important as when it was first proposed, and you have in its favour not only the opinion of the two committees of Congress on agriculture of 1830 and 1832, but also that of the committee of 1838, which, (saving the wonderful machine,) agrees entirely with the former ones.

Since this last report was made, I observe that the public opinion, on the subject of silk, has undergone a great change, and that it is now well understood that it is not a safe method to "improve first and learn afterwards." Conventions of the friends of the silk culture have already been held in different States. At those which were held at Baltimore, Philadelphia, and Harrisburg, I find that the Piedmontese reel has now obtained the general favor, and that improved reels are no longer talked of. I have read with great pleasure in the Farmers' Register, an excellent periodical, printed at Petersburg in Virginia, an eloquent speech, delivered by a gentleman who distinguished himself by his opposition to the filature system, and 
who was himself the inventor of an improved reel, * in which he passes a high and well me. rited eulogium upon that of Piedmont, which he says is superior to all others, and the general use of which he recommends to his fellow citizens. "All we want," says he, "to secure the precedence over the very factories of Italy herself, is the uniformity and excellence of reeling. By it we will kill every silk worm in Europe. The Italians themselves, to say nothing of France and England, will give us twenty or thirty per cent. more for our raw silk than they will for any produced in Europe." $†$

This seems rather exaggerated, besides that Italy exports and does not import raw silk, but it shows the opinion of the orator very different from what it was ten years ago, when he so gallantly attacked in a learned series of newspaper publications, the essays which I had then published, and in which I tried to inculcate precisely the same principles. He then proceeds to eulogise the Piedmont reel in warm energetic language, and in much stronger terms than any $I$ have used in the present work.

* Gideon B. Smith, Esq, of Baltimore.

† Farmers' Register, vol. 7, No. 2, February, 1839, p. 86. 
The learned gentleman, however, does not appear to have abandoned with his improved reel all his former prejudices; for he seems to think that the Piedmont reel works alone, independently of the skill of the reeler, and that the uniformity of the machine will, of course, produce uniformity of silk; in short, that it will produce of itself the excellent ran silk which he agrees with me in saying, that it is the only thing you want. I have no doubt that after reading this book, and particularly the Piedmontese Ordinance, he will sacrifice this last mistaken opinion, as he has generously done so many others.

The system of exportation, also, is gaining great favor; it has been recommended in the conventions of which I have just spoken; with what success I know not; but I have no doubt that its advantages will be ultimately appreciated.

I understand that other conventions, on the subject of the silk culture, are to be held. One is announced to take place at Hartford in Connecticut, some time in the course of next month. I am very glad to see that the intelligent silk culturists of that state are to take that subject 
into their serious consideration. Their country abounds in mulberry trees, and they produce annually an immense quantity of cocoons; it is in that neighborhood that it is particularly desirable to see a filature established. If their funds are not sufficient, they have at their door the great State of New York, so distinguished for its wealth and enterprise. But wherever the thing may be begun, I venture to predict that the first filature that will be established in the United States will, by quickening and encouraging the silk culture throughout the country, be able to supply itself with cocoons in sufficient quantity for its purpose, and in the end will prove to you an abundant source of riches, and lead you to that high degree of prosperity to which you are destined.

Although I have kept silence during the last eight years, I have not been inattentive to the progress of the silk culture in this country. I have remained faithful to the flature system, which I first recommended to you in the year 1829. The silk bill was only a development of that system, and a meass to carry it into execution. I did not ask for that legislative favor; it was offered to me, and I am thankful for it, 
though it ultimately failed. The renewed attention which has been lately paid to the subject of silk, and the great zeal which it has produced for the promotion of that object, has induced me, with the aid of the same kind friend who in 1829 assisted me in the composition of the essays, again to appear before you, and to communicate to you the knowledge that I possess of many important facts which I have acquired from practice and experience, and which you had not before the opportunity of knowing, because silk is yet, as it were, an exotic production in your country. I ask nothing of you but your good will, and if I have contributed in some degree to the further advancement of your hitherto unexampled prosperity, I shall have obtained the highest reward which I could ever have expected for these feeble efforts.

Accept, gentlemen, the assurance of my profound respect,

\section{J. D'HOMERGUE.}

Philadelphia, April 20th, 1839. 
T H E

SILK CULTURIST'S

\section{IMANUAI.}




\section{INTRODUCTION.}

To MAKE known the different species of the Mulberry tree, and point out those which are the most proper for the food of the Silk Worm; to exhibit, circumstantially, the best manner of raising these worms, of selecting the Cocoons, and of winding off the SiLK, especially at the time when there is a fair prospect of introducing the culture of this valuable article into the United States, are the principal motives of my engaging again on this subject.

To write upon any thing which is the immediate object of practice, is by no means so useless as the bulk of practitioners imagine. Agriculture and all parts of husbandry have long been arts of common practice, and yet will be subjects proper to be treated of, so long as they are capable of improvement; and even though they were not capable of being further im. 
proved, yet, it would be necessary to make public what was already known, that persons who have not the opportunity of being instructed, might be encouraged to begin their journey by having some directions of the road. In France and Italy, though the culture of silk has long been a practical art, yet, books are still written upon that subject, which have, without doubt, this good consequence, that they engage many in that business who would otherwise never have thought of it, because they had no rule to set out by.

The several publications which have been printed on this subject, have not explained even the present practice in such a manner as to extricate persons from the difficulties they would meet in their first trials, much less have they attempted to lay any foundation for future improvements; they were too concise to take in the various incidents that might embarrass a young practitioner, and, in many difficult parts, so obscure, that they seem to be only verbal translations, without any knowledge of the subject. As an instance of this, let any one read the de- 
scription given of the different reels invented; there is either a total neglect, or a total ignorance of its most essential movements. We need not mention the many material circumstances which are omitted in the hatching, feeding, and obtaining the breed of silk worms.

In the following treatise we have attempted to bring together the best information, and the most material facts which have been published by different authors, and, having ourselves many times gone through the practice of the different parts into which we have divided the subject, we have taken occasion to mention such improvements as we had actually tried, and also to submit such as we had reason to think would, on trial, be found useful: by these means attempting to impart what we already knew, and directing to such trials as might discover more than we knew, and striving to enter so far into the reasons of things, as might give some light to future discoverers.

If some should think we have treated the subject too minutely, we can only say, that this was owing to our observing that brevity in rules 
which were to be put in practice was often the cause of obscurity and error.

In short, it may happen that this treatise may be speedily censured, and slowly found useful; or it may, without either praise or cen. sure sleep in obscurity. But, while we are conscious of the intention with which it has been written, and are of opinion that it can, at least, do no injury to the cause which produces it, we shall not add solicitude about its credit, to the trouble it has already cost us. 


\section{PART.}

\section{CULTURE OF THE MULBERRY TREE.}

\section{CHAPTER I.}

OF THE DIFFERENT KINDS OF MULBERRY TREES.

The mulberry tree is the foundation of the silk culture, its leaves being the only food so peculiarly adapted to the worm which affords us its rich materials, that no other can be substituted for it, with any advantage, whatever authors may have stated to the contrary at various periods; experience has not only shown that the silk worm has no other aliment proper to its nature than the mulberry leaf, but it is also ascertained to be extremely hurtful to this delicate insect to change the quality of the leaf, as inexperienced persons in rearing them are too apt to do. The cocoons produced by worms uniformly fed with the same kind of leaf are always more beautiful, finer, richer in silk, and of a more delicate texture.

The first silk worms reared in Europe were fed on the black mulberry leaves, the only spe- 
cies then cultivated there, as would appear, although it was well known that the white mulberry was cultivated in Greece.

But the white mulberry, (Morus Alba, L.) was soon after introduced into all the temperate regions of Europe.

This mulberry tree offers three advantages: the leaf is earlier, and thus the care of the silk worm is not protracted too much into the hot season. It also gives a much greater abundance of leaves in a shorter period, and their quality produces the sort of silk most approved of by the manufacturers. But there is a considerable difference in the quality of the white mulberry trees. Count Dandolo, the great silk culturist of Italy, considers those as the best which grow in Lombardy, under the name of Fogtia Giazzola, of a middle sized leaf, dark green, and Foglia Doppia, of small leaf, of a dark colour, rather thick, more difficult to pick, and well calculated for the nutriment of silk worms, the leaf of which contains five different substances: 1 st, the solid, or fibrous substance; $2 \mathrm{~d}$, the coloring matter; $3 \mathrm{~d}$, water; 4 th, the saccharine substance ; 5 th, the resinous substance.

The solid or fibrous substance, the coloring matter, and the water, excepting what in part composes the body of the silk worm, cannot be said to be nutritive to that insect; the 
saccharine matter is, therefore, the only part which nourishes it, promotes its growth, and forms its animal substance; the more the leaves contain of it, in comparison with the other elements which compose it, the better is the tree adapted to the purposes of the silk culture.

The resinous substance is that, which, separating itself gradually from the leaf, and, attracted by the animal organization, accumulates, clears itself, and insensibly fills the two reservoirs, or silk vessels, which form the integral parts of the silk worm. According to the different proportion of the elements which compose the leaf, it follows that cases may occur in which a greater weight of leaf may yield less that is useful to the silk worm, as well for its nourishment, as with respect to the quantity of silk obtained from the animal.

Thus the leaf of the black mulberry, hard, harsh, and tough, with which the worm is fed in some parts of Spain, Sicily, Calabria and Greece, produces abundant silk, the thread of which is very strong, but coarse.

The white mulberry leaf of the tree planted in high lands, exposed to cold dry winds, and in light soil, produces, generally, a large quantity of strong silk, of the purest and finest quality.

The leaf of the same tree, planted in damp situations, in low grounds, and in a stiff soil, pro- 
duces less silk, and of a quality less pure an fine. The less of the nutritive substance the leaf contains, the more leaves must the silk worm consume to complete their development.

The result must therefore be, that the silk worm which consumes a large quantity of leaves less nutritive, must be more fatigued, and more liable to disease, than the silk worm which eats a smaller proportion of more nutritive leaves.

The same may be said of those leaves, which, containing a sufficiency of nutritive matter, contain little resinous substance, in that case, the insects would thrive and grow, but probably would not produce either a thick or strong cocoon, proportionate to the weight of the worm, as sometimes occurs in unfavorable seasons.

The black mulberry is the least advantageous for silk worms, because it produces a coarse silk, and much litter and moisture, which proceeds from its fruit; for these reasons, therefore, it ought not be used as a food for silk worms. The Spanish mulberry would have the same effect, if the silk worm were fed a long while with its thick and fat leaf. Persons skilled in the care of silk worms, do not touch this leaf before the moment of the greatest consumption, and at the time when they need to be filled. with substantial nourishment. 
The mulberry of Constantinople, (Morus Broussonetica, from the name of Mr Broussonet, a distinguished professor,) with a low and stunted trunk, very short branches, and leaves much clustered together; this species is difficult to be got, and yet more so to be propagated.

The red mulberry, (Morus Rubra,) which is indigenous to North America, and bears very large, numerous, wrinkled leaves, would also afford an excellent food for silk worms, if care were taken to engraft, or better yet, to inoculate it with rings of bark taken from the best white mulberry; it is small, not very succulent, of a dark green, shining, and contains little water, which may be easily ascertained by drying some of its leaves; otherwise we cannot recommend it as food for the worms.

There is another species of white mulberry which bears a middle-sized leaf, thick, and of a dark green color. When the trees are not exposed to a dry atmosphere, and are not in light soils, they bear a large quantity of leaves, but they are not then of such a nature as to afford much silk. It appears that nature more easily produces leaves abounding in nutritive matter, than those which produce resinous or silky substances.

Besides the varieties of the mulberry tree above mentioned, there is one, which, if we may 
believe the high commendations of it, is superior to all others for the silk worm. We mean the mulberry of the Philippine Islands, (Morus Multicaulis,) thus called by M. Perottet, and often confounded by amateurs in general with the ordinary mulberry of China, (Morus Alba Sinensis,) which, though resembling it a little in exterior appearance, differs materially from it in essential qualities.

This is a new and most valuable species of mulberry, which has been of late, the object of very great observation and experiment among cultivators in Europe, as well as in the United States. It is represented as possessing such decided superiority over all others, as to be speedily substituted for them in every part of the globe.

This tree was brought from Manilla, the capital of the Philippine Islands, to France in 1820, by M. Samuel Perottet, a celebrated naturalist employed by the French government. The Chinese inhabitants assured him that, to this tree, the disciples of Confucius are indebted for the prosperity and solidity of their empire, and that it is the only species used by them in the production of the finest silks, such as they weave into stuffs exclusively for their own use.

The Morus Multicautis is already propagating in many parts of Europe and the United 
States, and probably will be substituted for, and preferred to all other varieties. It is generally known in Europe, by a name derived from its origin, that is, the "Mulberry of the Phitippine Islands. In botanical language M. Perottet has called it Morus Multicaulis, on account of its roots having the property of putting forth many branches. Another eminent agriculturist, (Matthew Bonafous,) has thought it a better designation to call it Morus Cucullata, because the leaves have a concave form, inasmuch as the botanic description marks it by the following characteristics, Morus foliis cordatis, basi iniqualibus, vix lobatis, dentatis, amplissimis, cucullatis; mulberry with leaves heart-shaped, unequal at the base, scarcely divided into lobes, indented, very large and concave. Among the other qualities of this tree, it is affirmed that a less quantity of its foliage is required for the precious insect than of other species.

The lower branches of the Morus Multicaulis, are ordinarily straight and small, so that they bend easily under the weight of the leaves in the manner of a weeping willow, (Salyx Babyloniensis,) but those which grow from the crown of the roots attain often a height of six feet perpendicular. The fruit, which was unknown in Europe till 1830, consists of a 
small number of black pulpy grains, of which only a few arrive at maturity. It is said that these grains used as seed, seldom give a tree: resembling the one upon which they grew. To obtain one precisely similar, it is usual to resort either to inoculation or to cuttings; this last method is the best for the rapid propagation of this tree, because the cuttings take root: in a very short time and grow quickly. 'This species having its origin in the most northern part of the Philippine Islands, where the climate is much cooler than farther south, it would seem easy to naturalize it in all those countries where the white mulberry is cultivated. In fact, the experiment has already justified this hope. In the dreadful winter of 1829-30, in the coldest districts of Italy, where this mulberry had been introduced, only the smaller extremities of the limbs perished. It also withstood the hard winter of 1828, in the field of M. A. Eyries, at Havre. We are also informed that it has resisted the rigors of several winters uninjured and unprotected on the plantation of Madame Parmentier, of Brooklyn, L. I. Prussia, Bavaria, and Sweden are in possession of this very useful tree, and we are informed that it grows in those countries perfectly well. The experiments made in France, by Messrs. Audibert, of Tarascon, Barthere, of Toulouse, and Deslong-champs, of Paris, and those made by Messrs. Bonafous and others in 
Italy, on this interesting question, have confirmed all that has been previously asserted respecting the quality of silk produced by that plant. They have further proved, that the cocoons made by the worms fed with this quality of leaves, are rather heavier and produce silks comparatively finer, more elastic, and in greater quantity than those fed with the leaf of the common tree.

It has been ascertained,

1st. That the Morus Multicautis, does not require any particular soil, as exclusively suitable to its growth, but prospers even in a wet soil, which it seems to prefer.

2d. That it yields very little fruit, so that the leaves are more easily cleaned, and less matter of a fermenting nature is introduced into the body of the silk worm.

3d. That it does not rise too high, and yields a greater quantity of leaves which can easily be gathered by women or children.

4th. That it puts forth its thin, tender and soft leaves earlier than other mulberries, which permits the period of hatching of the silk worms to be anticipated some days.

5 th. That the roots possess the remarkable property of throwing up numerous small flexible stalks, without forming properly a principal trunk. 
6 th. That these stalks assume in a very short time a great length.

rth. That the leaves speedily acquire a remarkable development, and are promptly renewed.

Sth. That these stalks or branches strike root as cuttings, with extraordinary facility, without particular care, even before they have acquired a ligneous or woody consistence.

It may be proper here to give place to some remarks on the culture and uses of the Morus Multicaulis, by M. Perottet himself, which will be found very interesting.

"The Morus Multicaulis, which we noticed for the first time in the Annals of the Linnean Society of Paris, appears to have originated in the elevated regions of China; from whence it has been disseminated throughout the low plains near the sea shore. It is believed that it is cultivated in all parts of that vast empire where the raising of silk worms is an object of commercial importance. From Canton it was introduced into Manilla and all the islands of the Asiatic Archipelago, where it was only cultivated for ornamenting gardens. The Chinese are entitled to the credit of this introduction, who, in emigrating from their country, have from motives of industry, endeavored to multiply it, that they might render it useful to themselves in the new country of their adoption.

"6 The fortunate discovery of this precious 
plant, occurred in the garden of a Chinese at Manilla, who, after having informed us of its properties, and the important purpose for which it was used in his own country, yielded to our solicitations and sold us two bushes for ten Spanish dollars, assuring us that he had introduced it into Manilla, where it has been considerably extended.

"In August we brought it from Manilla, and first introduced it into the Isle of Bourbon, from thence into Cayenne and France. At a later period, it was sent from Cayenne to Martinique, and from France to Guadeloupe, and also to Senegal, where it has considerably multiplied. The numerous plants which are already disseminated in the various climates of Africa, America and Europe, have been all produced from the two bushes we procured at $\mathrm{Ma}$ nilla.

"Amongst the number of mulberry trees now cultivated by the Chinese, for the rearing of silk worms, the Morus Multicaulis appears to be the most esteemed of all, not only for the facility with which it is propagated and grows, but still more for the essentially nutritive property which the leaves possess. We have been enabled to verify this important fact during the five years which we passed in Senegal.

"6 Besides the advantages which we have already named, we may still add, that these trees are admirably calculated for forming regular plantations. They do not naturilly grow tall, or form any trunk properly so called; they can be $3 *$ 
placed very near each other without any injurious effect, and, by heading down the stalks annually, near the ground, a rich vegetation is produced, with a complete development of vigorous branches and leaves. And, finally, it is easy to multiply them by thousands from the roots in the course of the first year, and to form vast and regular plantations of them the second. A few years, then, are sufficient to obtain considerable fields of them in full vigor, and to support an immense quantity of silk worms with the greatest facility, as they are reproduced in a manner almost indefinite. Regular plantations of it can be formed without difficulty by planting the scions at a distance of six or eight feet from each other, a space sufficient for the extension of the branches, to facilitate the culture and for collecting the leaves; this last operation is so much facilitated by the flexibility of the stalks, that a child is sufficient for gathering leaves to feed a large establishment of silk worms.

${ }^{6}$ This species will be readily acclimated in Europe; because it originated in an analogous region as to climate, to that which we inhabit. It appears not to suffer from the excessive cold of the northern, nor the intense heat of the intertropical regions; for the plants deposited in the gardens of the government at Cayenne, acquired in the space of eight months a truly remarkable development, and at the time of our departure from that colony, in June, 1821 , they were clothed with leaves of an ex- 
traordinary size. Those, also, which we cultivated at Senegal, although situated under a dry and scorching sky, and planted in an arid soil, afford an appearance suficiently satisfactory, but they had acquired less development in all respects than those which had vegetated under the damp climate of Guiana. It appears expedient then, that plantations of this inulberry should be made upon a humid rather than a dry soil, to obtain in all respects, a satisfactory result.

"Besides, this mulberry stands the most rigorous winters. We saw on our arrival at Havre, in July last, in the field of $\mathrm{Mr}$. A. Eyries, plants, which had withstood in the open ground, the winter of 1828 , and which appeared vigorous and beautiful."

Thus much M. Perottet. Let us now hear the testimony of Mr. Poiteau, on the same tree, in the Annales d'horticulture, 1830.

"By the information which we receive from all quarters, it appears that this mulberry is destined to replace the common white mulberry every where for feeding silk worms; its property of continuing low and bushy, so that the leaves can always be gathered without a ladder; and the large size, abundance, and tenderness of the leaves, cannot fail to give it a decided preference. It has been sufficiently ascertained, that they are eaten with avidity by the silk worms, and that the silk which they form is of the first quality. This mulberry has not suffer- 
ed in the least, from the rigors of the last severe winter.

"The zealous traveller, who has given to France, America, and A frica this precious plant, has acquired a just claim to public gratitude, and it is not only easy, but proper, to give him at this time a proof of $i t$, by affixing his name to the tree which has given him celebrity, and which will contribute so much to the prosperity of French industry."

Dandolo, or Moretti Mulberry.-This is a new and most valuable species of mulberry tree. It was first discovered by M. Moretti, Professor in the University of Pavia, and from a single scion he had, in 1826, multiplied them to one hundred and twenty thousand. The tree is presumed to be strong. The fruit which is at first purple, becomes at maturity, perfectly black. The leaf is oval, sharp pointed, entire, cordate at the base. It is thin, smooth on the under, and especially on the upper surface, which is of a beautiful and rather deep shining green; it is not near so thick as that of the large white mulberry called in France "The Admirable," in consequence of its remarkable beauty and qualities, and is thinner than those of the Spanish mulberry. It is neither wrinkled nor plaited. It is in general nearly eight inches wide, and ten inches long. This species will be most profitably cultivated in 
the form of a hedge, and from the great size of the leaves, they are gathered with the greatest facility. Its superior quality has been proved by the experiments of Messrs. Gera and Count Dandolo, who assert that they produce silk of a more beautiful gloss and of a finer quality. This last species of mulberry tree is, we believe, not much known in this country. We should like to see its introduction, but it is probable that the Morus Multicaulis will prevent it. 
PECULIAR SUBSTANCE IN THE MULBERRY LEAF, AND THE ANALOGY OF ITS CONSTITUENT ELEMENTS WITH SILK.

There is no doubt, some peculiar substance in the mulberry leaf, which is not to be found in any other vegetable production. Experience has shown that the leaves of all the different kinds of mulberry which nourish the silk worm, have a granulous surface covered with a great number of minute tubercles resembling glands, which seem to contain the odour which the leaves exhale. This observation very early formed the subject of some examinations by several chemists, and they have been enabled to ascertain the existence of a matter altogether peculiar to the leaves of the mulberry, having no relation to the general principles of other vegetable productions.

Thus, then, if merely by the substance of this neutral matter, the leaves of the mulberry are distinguished from all other vegetables, if it be a fact that the silk worm feeds exclusively on these leaves, and that no other food enables it to make cocoons of good quality, there 
seems, then, reason to believe that silk is only a metamorphosis of the matter in question.

In our last voyage to France, in the summer of 1837 , a friend of ours communicated to us the observations on the species of mulberry, re. cently discovered by Professor Bafrau Delille of Montpellier, which he calls Morus Nervosa. It is said, in fact, that the transparent edges are formed by the extravasated gummy and resinous matter of which the silk is constituted, and that the worm fed with this sort of leaf gives a silk of finer quality and greater quantity. No doubt that an immense service could be rendered to the culture of silk, if it could be ascertained by an exact and thorough analysis, what is its actual composition. Unfortunately, this precise knowledge is yet wanting. Messrs. Berthollet, Fourcroy and Chaptal, have declared that silk has much analogy with the tissue of horn, and that by distillation they have obtained from it carbonate of ammonia, and a large portion of oil. Roard, in his excellent menoir addressed on the subject, to the National Institute of France, (see vol. 22, Decreusage des soies-ungumming silk, gives an analysis conformable to the observations of the three celebrated chemists above named. He demonstrates that the yellow silk contains of gum, from twenty-four to twenty-five per cent.; of coloring matter, which is 
a resinous substance, nearly solid at twelve degrees of the centigrade thermometer, and entirely liquid at thirty degrees, its proportion is fifty-five to sixty per cent.; of wax, which melts at between seventy-five and eighty degrees, it is insoluble in water, and dissolved easily in the alkali or in soap; this part is no doubt what forms the exterior brilliancy of the silk; this matter is found in the yellow as well as the white, and forms from 1.200 to 1.250 of their weight; and a volatile oil having a strong and disagreeable odour, and which, by itself, resembles the essential oil of anise, or of any other vegetable matter.

By these characteristics, we may conclude that silk is of an intermediate nature, between vegetable and animal, which will correspond with the qualities discovered by the above chemists.

Another proof that the quality of the leaf has an important influence on the quality of the silk, is found in the observations lately made on the leaf of the Morus Multicaulis. Any one can satisfy himself that these leaves are more abundantly and entirely covered with those little glands than any other quality of mulberry, and consequently they contain a larger quantity of that nutritive substance spoken of.

After having treated of the different species 
of mulberry bearing leaves, proper as food for the silk worm, we will treat of the culture of that plant. Here we must acquaint the reader that a great part of the method which follows for raising mulberry trees is drawn from some of the best authors who have written on that subject, for the climate of Europe, to which every thing found in the writers of this country, which seemed useful, has been added, and some things which were deduced from our own experience; nor have we omitted giving hints of such improvements as, though we have not had time and opportunity to try them all, seem to us reasonable, and will, perhaps, upon experiment, be found useful. 


\section{CHAP'TER III.}

OF THE CULTIVATION OF MULBERRY TREES.

This Chapter embraces the following heads:-

1st. The manner of gathering and preserving the seed. $2 \mathrm{~d}$. The soil proper for the raising and plantation of Mulberry trees.

3d. The various modes of propagating the Mulberry trees.

4th. The planting out of the young Mulberry trees.

5th. The grafting and budding Mulberry trees.

6th. The culture, pruning, and management of the trees. 7th. The diseases of the Mulberry trees.

Oun principal objects in adopting the above distribution and arrangement has been to show, first, the manner of obtaining the greatest possible quantity of leaves during the proper season for raising silk worms; second, of securing the vigor and the health of that insect, on which depends the richness of the crop; both tasks to be accomplished with the least expenditure of time and money.

We have adopted the above plan as the most eligible for condensing the directions which appertain to this part of our subject, being convinced that one part of them only is necessary 
and unchangeable in any climate or latitude where the plant can grow. But the task must ultimately be left to the discernment of the agriculturist himself to judge, what circumstances and practical means are necessary, by the nature of their own soil, in the order of seasons, and with their adopted modes of tillage. Therefore we could not select a better model than the method of the Count de Verry, in his treatise on the mulberry, and that of the celebrated Doctor Antoine Pitaro on the same subject. We have borrowed at least, those precepts, which we thought must be strictly observed. On the other hand, not mistrusting our own knowledge and experience, acquired in one of the richest silk districts in France, we have attended to such selections and comments as we believe to be interesting, and may prove useful.

1st. Of the manner of gathering and preserving the Seed.

The mulberries whose seed you intend to keep, should be gathered perfectly ripe, this you may know by their beginning to fall from the trees, by their softuess, and, if you examine nicely, by the kernel being completely ripened in the small shell which encloses it. You should not choose the fruit of those trees which have been stripped of their leaves that year, or even 
the year before, if you can avoid it; the berries of the white mulberry which incline most to a dark color are the best. It is a good method to shake the tree moderately every day from the time that the fruit begins to ripen, for mulberries do not all ripen together.

These mulberries should be laid thin on a clean table, in some airy place, for four or five days, in order that they may attain their full ripeness, and should be removed and stirred every day for fear of heating or rotting; especially if they lie thick upon one another; after this let them be soaked in water until they can be easily bruised with your hand, and thus dissolve and separate the grain from the pulp; separate the seeds afterwards by washing the mixture with several waters; stir it briskly, the seed will sink, in three or four hours, to the bottom, and the pulp swimming at the top, may be poured off with the water. The seed should then be taken out, spread on cloths, and dried in the shade or in some airy place, stirring it often for fear of the heat injuring it. When perfectly dry it should be put into a tight vessel and kept in a dry place.

This and other processes in relation to a provision of seed, should never be neglected by good culturists, to guard against the chance of employing old or poor seeds : which danger 
is obvious, unless they can trust to horticulturists and gardeners who follow that business with diligence and reputation.

In the more temperate climates of Europe, it has been found necessary to select good trees, well manured, and hardy, in order to have sound and well-ripened seed; and in any climate, it would be useful to have a few trees of the best growth peculiarly set apart for seed. But, if, for want of better, you are obliged to make use of the seed from trees whose leaves have been pulled for the worms, let it be from those trees which were last used, viz: when the worms are in their last age, at that time they have their fruit pretty well grown before having been stripped, for the mulberries do not fill when the leares are pulled too early. If the berries are ripe on any tree whose leaves you have occasion for, it will be convenient to shake the tree moderately every time before you pull the leaves; thus you will both save the fruit for the seed, and prevent it from mixing with the food of the silk worms; and on this last account you should preserve for seed those trees which are so greatly loaded with fruit as to have but few leaves, since it would be almost lost time to pull the leaves; and they would be so mixed with the mulberries as to occasion a great deal of litter among the worms; and if 
they were so ripe as to shed their juice on the leaves, it would make them unwholesome food.

\section{2d. Of the Soil proper for raising Mulberry trees.}

The ground for raising mulberry trees either from seed, layers, or cuttings, should be a rich, loose soil, mixed with much mould, and inclining to sandy, in order that the tender new formed roots may be the better enabled to extend in it. A small depth of soil will be sufficient for these, especially those raised from seed, but it ought rather to have gravel under it than any sort of stiff clay, which might preserve humidity, and chill the young plants.

Though a small depth of soil is sufficient for raising trees which are to be transplanted, yet, when they are raised where they are to stand, (as will be shown under the head of raising mulberry hedges, or when they are transplanted where they are to remain, a deep soil is best, because mulberry trees shoot downward, with very long tap-roots; it is true, indeed, that when they have taken root they will live, and throw out abundance of leaves, even in a bad soil, but then those leaves are not so good to feed the worms, especially, when the trees are planted in low watery grounds, they then afford but bad leaves, though they may have a greater quantity; for, 
as they abound too much with moisture, their leaves approach to the nature of those which grow on the suckers of the mulberry trees, and endanger the silk worms which feed on them, especially if they had been used to better leaves before.

\section{3d. Of the various modes of propagating the Mulberry trees.}

There are three principal methods of raising mulberry trees: 1 st, by sowing the seed of the berry; 2d, by layers depending upon the mother trees, till they have taken root; 3d, by branches separated from the mother tree, and therefore called cuttings. We shall begin with the method of raising them from seed, as it is both the surest and most effectual way to procure great numbers; and numbers of small trees will answer the end of a few large ones.

1st. To raise Mulberry trees from the seed; choose some part of an inclosed garden which has a good exposure to the sun, and is protected from the north wind; the ground should be ploughed the preceding fall, and again ploughed two or three times in the spring and made light and friable, two or three dressings of dung, not too much decomposed, and well ploughed in, 
would be of essential service; then lay out the ground in beds, a little raised above the surface of the earth, of what length you may think proper, but not above four feet broad, that they may be conveniently weeded.

The best time for sowing the seed is in February, March, April, May, even in June, for it depends upon the climate. The sooner it is done in spring, when frosts of any continuance are no longer to be feared, it will be so much the better, as the plants by coming up early, will have time to get strength, and be the better able to bear the ensuing winter. As the plants require, according to the difference of climate, twelve to fifteen days, with sound seed and good weather before they begin to rise, we think it is a good rule to sow the seed about twelve or fifteen days before the time the mulberry bud begins to open, (which may be a sort of general direction for any climate, ) although no alarm need be felt if it should be later, as was the case in France, in 1825, when three weeks elapsed before the plants appeared.

Take, therefore, the seed which you have kept, and if it is too dry, or if the season is too warm, steep it for one night in water, in order to induce the germination, then place two seeds at most in each drill made in the beds prepared as above, one inch and a half deep in 
a heavy soil, and two inches deep in a light one, and five or six inches asunder, and covered with earth; should the weather be dry during, or shortly after the sowing, the beds should be watered immediately after the seed has been sown, and at least once every two days in dry weather, particularly towards the time when you expect the seed to spring up.

While the earth appears moist you need not water them, since it is very apt to thicken the surface of the ground, and make a crust gather on it, which prevents the springing of the young plants, and therefore when you water them, it should be with a watering-pan whose rose has very fine holes, or else you should have two or three light bundles of osier, straw, or rushes, which you should lay upon the several parts of the beds as you water them. If any frost happens, or the weather be very hot when you sow the seed, it is proper to cover the beds with some straw for four or five days; this will protect it from either cold or drought, according as the season is, and also, from being scratched up, and picked by birds; above all, it is requisite, often and with the greatest care and gentleness, to stir up the earth, clearing it from all weeds, which would impede the growth of the young trees. For this and other reasons, we would advise to make the drills run 
across the bed, and not lengthyays, because the: weeds can be more easily seen and destroyed.

If the seed has been preserved with some of the pulp about it, you may sow it in a drill in the following manner: first steep it in water till it becomes a soft pulp, then make drills across your beds as before directed, only somewhat deeper; then, having some small ropes of hay, about as thick as bulrush, fix them with two pegs directly over each drill; take now the moist pulp, and rub it along the rope, so that the seed mily stick here and there upon it, then thrust the pegs down, so that the rope may lie in the bottom of the drill, and eover it with earth, after which, you may draw out the pegs. This, although a rery easy method, is not so good as that above described, because if the seeds are too close, they will not germinate well, or oblige you to pull up some of the trees if they rise too thick. You may also sow the seed at its maturity, well cleaned, free from any animal or regetable substance, and mixed with so much fine sand as may serve to separate the grains: thus you will gain almost a year in the growth of the trees; but, as this is done in the summer, the beds should be duly watered, and the plants, when winter comes on, should be protected against frost with straw or some other covering, which should not lie too close 
for fear of suffocating them, and in mild weather should be taken off; dry leaves are best for such purpose.

If the nursery ground has been well attended to, during the first year, the little mulberry trees may be taken up the next spring, to be re-planted either in another nursery, in order to become large trees, or in the field, to make hedges of low stature. But if it is preferred to leave them longer than the spring, in order that they may gain greater strength, it is useful to cut those stems close off to the ground, by the means of such a sharp and well-edged nipper, as will not shake nor disturb the roots. The new growth will acquire additional vigor from this operation, and there will be obtained the second year, a mulberry tree four or five feet high, and sometimes even more.

2d. By Layers. - Though the mode of raising mulberry trees from seed, is the most advantageous, yet, where large plantations are to be suddenly raised, all other methods may be at the same time used. Layers may be made from low trees, and from shoots, which are sometimes produced plentifully from the roots; but the method of making layers being generally known, needs not be particularly insisted on; the branches now are bent and pinned down in the earth, 
with crotchets of wood, leaving only their extremities above ground, and in this situation they subsist on the mother tree, until the parts which are under the earth have formed roots sufficient to maintain themselves, and then they are to be separated from the trees and planted. out.

These layers are more certain of growth than cuttings, and do not require such watering while they depend on the old tree; but then, this dependance makes them lazy in putting forth roots, with which they often appear but moderately furnished, when you come to separate them from the old tree; therefore some agriculturists recommend to twist a piece of brass wire about the parts laid under ground. The theory upon which they built their improvement, is, that as the branches of trees are produced generally at acute angles from the stem, and tend upwards, so that the roots are produced at acute angles, but in an opposite direction, downward. The ligature, therefore, checks the motion of the sap, and turns it into that direction which is proper to produce roots, and experience seems to have convinced them of the truth of this theory. We should think that a ligature of lead or of cord, may be found fitter for that purpose than brass wire, as they will give way to the growth of the branch, 
and not cut through the bark, as the other appears to us apt to do. The spring is the most suitable season for making layers, and in two years they will be fit to transplant. It might, however, be of use, if, at the end of the first year, you should begin to wean them from the mother tree, by cutting them off four inches from it; and putting tar upon the cut, this might force them to put forth roots for their subsistence.

Shoots often spring from the ground, about the roots of trees, which have their heads torn by winds, or many of their principal branches lopped off, this may be promoted by digging and opening the ground about the roots of such trees, but, though such suckers spring out of the ground, it is needful to their well rooting, that they should be bent down, and have good mould laid on their lower parts, in the same manner as has been described for branches laid down.

Besides the foregoing methods, we think proper to mention, that mulberry trees may be propagated from parts of the root taken up, the more length they have will be the better; these may be laid horizontally, about two or three inches under ground, in a loose fine mould, and placed in lines to produce hedges, as is generally done with cuttings; they may be planted early in the spring, and will soon shoot forth 
plants, which, being at first sufficiently rooted, are less liable to failure. The ground, however, should be watered in dry weather, both before and after they have sprung, because the roots lie near the surface, being so placed in order that they may put forth plants above ground earlier.

3d. By Cuttings. - The next most easy and quick method of raising mulberry trees, is from cuttings; and in this method, though you cannot so readily raise the same number as from seed, yet you have the advantage in the quickness and strength of their growth. The mulberry is more easily propagated from cuttings in moist and temperate climates, (particularly. the species called Morus Multicaulis, as we have already said,) than in those which are extremely. hot and dry; for the branches which are separated from the mother trees having no roots to subsist upon, require to be plentifully supplied with moisture, in order to keep them alive till the roots are formed, and where the clid mate denies this, the defect must be made up with watering.

The soil chosen to receive the slips or cuttings: of the mulberry tree, should be prepared much in the same way already described for the seed. A two year old branch of a mulberry tree: 
which has wood of four or five years at one end; must be selected, and the extremity of the old wood must be interred to the depth of about ten inches. The branches must be taken off from sound and well grown trees; and in order to facilitate the shooting of the roots, two or three incisions must be made in the joints or knots of the old wood, from which the roots always put forth.

The best time for planting cuttings, is as soon as the leaves have fallen in autumn, so that the parts which lie under ground may be prepared to put forth roots the spring following; for, if they are planted in the spring, they will mostly fail for want of roots to supply the large quantity of nourishment which the leaves will soon require. The soil in which the cuttings are to be set,-should have been dug, broken and weeded some weeks before; and if there was a necessity, enriched with some good loam, dung, sand, or other manure, so as to make a fine loose mould proper for the young fibres of the roots to shoot into. In this make furrows of about one foot in depth and two in breadth, sloping from the edge to the bottom of the furrow, and of the length which you design for your hedges; the distance between each furrow need not be above four feet, if you design future plantations, by taking away and transplanting one or 
more of the intermediate hedges; but if no more hedges are planted than you intend to stand in the same places, then the trenches should be four or five yards distant. The ground between the trenches may be cultivated with any low vegetable that will not overshade the young mulberry plants. The trenches should run from north to south, for reasons given in speaking of the form of a mulberry plantation.

When you would plant the cuttings, first fill the trenches about one third with some of the fine mould taken out of them; then begin to lay in the cuttings, by placing their main stem as flat as you can on the bottom of the trench, and turning up the small branches on each side so that their ends may stand above ground from three inches to a foot, as they can be conveniently bent and turned up; for which purpose place that side of the main branch undermost which has less twigs growing out of it; you may bend the small branches, and make them yield as much as you please, they will not grow the worse.

The cuttings must then be covered with a well manured and friable earth, and the end of the branch which rises from the soil must be cut off at the third bud from the surface. If rains should not frequently occur after the plantation is finished, it would be necessary to water the plants often. 
These cuttings, thus managed, will in the first summer put forth shoots of about eight or ten inches in length, more or less, according as they have thriven; and at the same time they will strengthen themselves with roots, so that if they have made shoots, and preserved their leaves till fall you need not doubt of their growth.

In the ensuing spring and summer, although they will then be more out of danger, yet you should continue to water them in very dry weather, and they will this year put out shoots, often a yard in length, and furnish themselves completely with roots, so as to be fit for transplanting in the fall. This method therefore, of raising mulberry trees, we take to be as advantageous and expeditious as any that can be pursued, and if cuttings thus managed fail to grow, at least in a temperate climate, it will be owing either to want of watering, weeding, or a good soil.

We would recommend, before the cuttings are put into the ground, as above directed, to dip the part that is cut in tar or melted pitch; because when moisture insinuates itself where a branch is cut, especially in the mulberry tree, it generally makes the bark part from the wood and grow rotten; besides, the tarring over of the cut will, as we think, prevent it from taking in a large quantity of crude and improper 
juices, which will now be forced to strain through the bark, and prepare the shoot to put forth roots.

'The method here given for raising mulberry trees from cuttings, may perhaps be advantageously applied to the producing of nurseries from many other trees, as well as from the mulberry. All that seems necessary for producing trees from cuttings, is, that the part which is under ground should, as soon as may be, acquire the nature of a root, so as to feed the branches. Wherefore the less any particular tree is apt to strike root from cuttings, the more of the branch must lie under ground to make up the defect, and the more should such other artifices be used as will make it put out roots; for otherwise, though a branch may bud and keep alive during the beginning of the summer, yet when the leaves are expanded by the advance of the season, they require a greater supply of sap. and the branch will often, for want of due nourishment, decline by degrees, and at last die.

4th. Of planting out young Mulberry trees.

Mulberry trees raised from seed, or from parts of the roots laid in the ground, may be transplanted after having acquired the thickness of an inch and a half in diameter. Though 
some authors mention that mulberry trees may be transplanted in the spring, yet the surest season for doing it, is just after the leaves have fallen in the autumn; for the dry weather, which often happens in spring, before the roots have settled and begin to draw nourishment, will much endanger and often kill the plants; but, when planted in the fall, this is avoided, and there is then nothing to be feared, except a severe frost soon after planting, but this may be prevented from doing any harm by laying some fresh litter, straw, or the like on the ground, over the roots.

The pits for setting out should be prepared and always left open beforehand, in order to fertilize the soil by the airing or contact of the ambient atmosphere, which, as is very properly supposed, has its share of influence upon the vegetative property of such layers or strata of earth as have thus been mellowed. It is in this view, also, that a judicious horticulturist will take care to set apart the upper and best vegetable earth, and lay it on the roots of the sapling.

There are two methods of planting out the young mulberry trees, or of forming plantations, viz: 1st, in hedges, or 2d, as standard trees.

1st, The planting in hedges affords three important objects: supplying food for silk worms; keeping the trees low, so that the leaves may be gathered from the ground by children; and 
furnishing a good and almost never ending fence for gardens, meadows, \&c. In transplanting young trees for hedges, they should not be pruned till the second year, or at least the third, the tops should be cut off and the side branches trained laterally with the hedge, by interweaving them. 'The white mulberry forms an excellent live fence, and, when once established, is probably the most permanent of any other.

2d, As standard trees. This operation, as well as the one already spoken of, requires considerable diligence, both in taking up the trees, so as not to spoil or damage the stem, and also in re-planting them. It is necessary to cut carefully, with a sharp and well-edged nipper, all the branches of the young tree close to the stem, without, in so doing, inflicting any serious wound upon the tree; all the injured roots must be cut off. The trenches to receive these trees vary from four to five feet in diameter, according as the soil is more or less strong. In stiff and argillaceous ground the trench ought to be larger. The depth should not exceed two feet, in order that the roots, not being too deeply buried, may feel the influence of the sun's rays; horse dung, not too dry, and sheep dung make the best manure and may be put in the bottom of the holes, or some fresh mould. It would be 
desirable to ascertain the exposition that the plants had in the nursery, which may be easily discovered by the size and the number of its roots, which are generally more considerable on the south side of the tree, as well as by the size and the distance of the rings or circles which form at the forks of the branches. Directed by these signs, one may give to the tree the same exposition which it had before its removal, by attentively turning to the south the largest roots and circles.

It will be a good precantion to put a stake, firmly driven into the ground, by the side of each tree recently planted, and tying the tree to it. The wind will thus have less power to disturb the tree and draw the roots out of their proper position. Three or four dressings a year with a hoe, might be of essential benefit. Freshness and strength are communicated to the tree and to its roots by this attentive tillage, and the benefit is so visible, that even an unpractised eye will at once distinguish the plantation of the industrious from that of the negligent proprietor.

5th. Of grafting and budding Mulberry trees.

Bonafous observes that there is a difference of opinion in regard to this method, as some think it advisable to graft the young trees, 
whilst others prefer to leave them in their natural state. Count Dandolo appears to be of the latter opinion; let us hear him on this last point.

"I have fed, though with difficulty, a number of silk worms on the leaf of the wild mul. berry tree alone. This leaf is scarce, because even the hedge mulberry trees are grafted.

${ }^{66}$ The cultivator, finding that the grafted mulberry yields more leaves, is always anxious to graft as soon as possible, which has prevented my making the experiment on a large scale. I have however, ascertained the following facts.

${ }^{66} 1$. That $14 \frac{1}{2} \mathrm{lbs}$. of wild mulberry leaves, weighed when just gathered, without sorting, will produce a pound and a half of cocoons, while it requires $20 \frac{3}{4} \mathrm{lbs}$. of the leaves of the grafted mulberry to make the same quantity.

66 2. That $7 \frac{1}{2} \mathrm{lbs}$. of cocoons, proceeding from worms fed on the leaves of the wild mulberry gives about fourteen ounces of exceedingly fine silk; whilst generally, the same weight of cocoons, managed exactly in a similar manner, but of which the worms have been fed with leaves of the grafted mulberry, yields only eleven or twelve ounces of silk.

663. That the silk worms fed on the wild leaves are always brisker and have better appetites."

The celebrated Doctor Antoine Pitaro is of 
the same opinion on this subject. "The black or white mulberry transplanted from the nursery," says he, " is the true and natural mulberry, and it is for that reason that it is called wild mulberry; yet it is in our opinion preferable to the grafted mulberry, because its leaf, compared to the grafted mulberry, yields, at equal weight, a greater portion of nutritious and resinous substance, and less of the fibrous substance."

It would appear from what we have stated, that we prefer the culture of the wild mulberry; however, before coming to a conclusion the agriculturist should weigh the following observations attentively.

1. In the vast class of the wild mulberry plant, there are varieties of an indifferent quality, which yield few leaves, those leaves much indented, and the branches thorny.

2. Some give a great quantity of fine sound leaves, scarcely distinguishable from the grafted mulberry leaf.

3. The wild mulberry tree with sound leaves and of a good quality, may be grafted on the wild mulberry tree of the inferior sort, with indented leaves.

4. As it is the nature of the wild mulberry to have a great quantity of small branches, and grow bushy, it should be well pruned and thinned, which will also strengthen the tree. 
5. The hedge rows of wild mulberry trees should all be grafted with the best sorts of the wild mulberry, and they should be planted in every spot where they are not likely to injure any other production.

We may have enlarged too much on this subject, and we must conclude that before the culture of the wild mulberry tree shall supersede the grafted tree, experiments must be made for many years, from which alone exact calculations and unerring practical comparisons can be deduced; therefore we will proceed to give the best method of grafting mulberry trees.

We are sorry to be in opposition with one of the most illustrious writers of Lombardy, the Count Verri, who recommends very highly the engrafting by the shoot as the surest and most advartageous mode. Experience has, however, proved that the branches proceeding from such a graft will never acquire much strength, and moreover, the smallest storm of north wind will suffice to break them. The engrafting by rings is better adapted to the tree, and gives to its branches more strength to support the force of the wind. Scions of the preceding year are the best adapted either to inoculate or to graft the trees, and the time most suitable is the month of May, on a dry and calm day, for rains and high winds are injurious. 
The branches from which the rings are to be taken should be cut from the tree in the first fortnight of April, when the sap has ascended to the limbs, to the very extremities of the branches. After having cut them off, it is the custom to bury them in the sand, a little moistened, for fifteen days, in order to render the bark more flexible and easier to be removed. The operation of inoculation is very easy, and may be learned in three hours' exercise, after seeing it done by an able inoculator.

During the whole year in which the inoculation has been performed, care must be taken not to suffer any other shoots to grow than those which are inoculated, and which are intended to form the crown of the tree. In the spring of the following year the engrafted branches should be trimmed, leaving three eyes between the place of inoculation and the extremity. This operation surprisingly concentrates the strength of the tree, which, the same spring, puts forth very flourishing branches.

Generally, only three branches are left, which form, with their shoots, a fine crown or top to the tree. It is always useful to cut off the branches that have taken an ill direction or become thorny, or when they weaken the plant too much. This ought however, to be done only before the sap has recommenced 
its circulation; at the same time all the little branches or extremities should be lopped off that have perished by the cold or any other accident.

6th. Of the culture, pruning and management of the trees.

Few persons bestow much trouble on the mulberry trees designed for silk worms; and yet it is certain, that the leaves will be more wholesome and the trees thrive better by culture.

In some countries of Europe which produce silk, the grown up mulberry trees are left to themselves; in others they are more or less pruned. Both these methods are more founded upon custom than upon sound principles of rural economy. Three things ought to be kept in view :

1st. The quality and the quantity of the leaves. 2d. The duration of the tree.

$3 \mathrm{~d}$. The facility and the certainty of the gathering of the leaves.

Any method adopted, without regard to these primary conditions, is dangerous, or useless. The clipping of the trees contributes undoubtedly to render the leaves more numerous, and larger; but if practised without judgment and necessity, or done at an improper time, or imperfectly, the leaf not only becomes 
less nutritious, but diminishes in quantity; it fills the tree with wounds and scars, and weakens it; much caution is therefore recommended in pruning, in the first year that the leaves are plucked; and the pruning must be so executed, that by an equal distribution of the sap, there may be a perfect equilibrium in all parts of the tree. Consequently, ten days at least after the fall of the leaves, or after they have been pulled off, all the branches damaged in that operation, and all the dead ones, ought to be lopped off, as well as those the vegetation of which seems too low ; and those which, on the contrary, are too luxuriant, ought to be restrained in that propensity, or turned up in a different direction, to diminish the sap; the tree ought not to be left to grow to an excessive height, nor to spread too far; the branches which obstruct the development of the head, or hang too much down, should be shortened; and lastly, those that have been thrust out of their natural direction, during the gathering of the leaves, ought to be set right again; and with regard to the rest, the good sense of every cultivator will direct him how to form trees the most beautiful as well as the most productive.

In regard to the preservation of mulberry trees and mulberry hedges, much depends on the method of gathering their leaves. It should 
be done with the greatest care, with the view of causing as little injury as possible to the plant, of preserving it in good condition and proper vigor, so as to satisfy the voracity of our precious insect. It is essential that all the leaves should be pulled off; for, if any remain on some branches, they attract the sap, whilst the naked. branches are incompletely nourished. Begin by pulling the leaves of the hedges, if you have any, then proceed to the young trees, whereby the latter gain time to come into fresh leaf, and the sooner, in proportion as the leaves of the older trees are more fully grown, more nutritious, and more suitable to the worms in their progress and advanced ages. The stripping of the leaves should not be begun before the disappearance of the dew, and ought to be ended before the setting of the sun. The hand should move from below upwards, in order to avoid pulling off the buds, and an inverted force and direction that might pull off the eyes and break and mutilate many parts.

It is never proper to climb upon a tree which is not strong enough by age or by nature; for the motion is more injurious than the additional weight; the best way is to use the double wheelbarrow, or step ladder.

The bags employed to contain the leaves should have a hoop at the mouth to keep them 
open, and be furnished with a hook, that they might be hung on the branches; when filled another should be at hand, and care taken to keep them sheltered from the sun.

Mulberry leavès are frequently much soiled in summer with what is called honey-dew, a viscous matter. This is a phenomenon not yet accounted for; it results from insects, from the plant or from the clouds. Sauvage, Bonafous, Count Verri, Dandolo, Pitaro, and other eminent agriculturists, have said much about it; whatever it may be, the honey-dew is poisonous to the silk worms, and leaves, thus stained, should be thrown away. Rusty or partly dried leaves have not this inconvenience, because the worms either refuse them or they eat only the healthy portion; if wet by rain, they induce disease, and you have to dry them by spreading them in parcels on a clean linen cloth, in a dry and airy room, stir them often with a wooden fork, shake the cloth, and the leaves will soon dry; dusty leaves must be also cleansed before feeding the worms.

\section{7th. Of the diseases of the mulberry trees.}

Every species of tree is liable to some peculiar malady; and when the interest of the proprietor is awakened by the value of the object, he applies the means of cure already known, or 
has recourse to some remedy of his own, which he thinks the nature of the case requires. It would be too long to relate here every thing which has been published on the diseases of mulberry trees, we will only expose those most fatal to them, and particularly one which has been observed epidemically ruinous to plantations. The following literal translation of a case and cure of that kind, by Count Verri, will at once indicate the nature, the danger, and the treatment; observing, however, at the same time, that the same disease may originate from carious or gangrenous spots at the extremities of branches instead of being only confined to the root, as in the following instance.

${ }^{6}$ A mulberry which $\mathbb{I}$ had planted and taken care of during twenty years, offered all at once, alarming symptoms; the leaves grew small, and the greater part of them withered; others were yellow, and dropped off long before the autumnal period. There not appearing any local defect, I ordered the ground to be opened, and the roots to be searched. I found that a few had been gnawed by moles, and others were diseased. I had them all taken off, I renewed the earth, mixed it with manure, and curtailed a little the sound ones. But this was not all, I had all the branches pruned off except the principal ones, which were shortened only, and I made, 
with a very sharp knife, several deep, longitudinal incisions, to the places from whence a thick fluid oozed out, taking care to detach all the black parts. This operation, by facilitating the flowing of that humor, saved the tree from gangrenous ulcers, which would have been subsequently formed. All this was done in the month of September, the next year the tree still kept in a hopeful condition, although very weak; but in the next it perfectly recovered its vigor and luxuriant vegetation. I had occasion to repeat the same method with several other trees, and always very successfully, although this last was done in the month of May. My neighbors, being advised by me, have experienced the same success. It is unfortunately but too true, that if, in a row of fine trees, one alone be attacked, the next one will soon follow, show the same disease, and die; in which case, having dug up the dead tree, it will be necessary to open a large and deep ditch between that and the next trees, (to intercept contagion,) and all the rest will be preserved." -Verri, pp. 81-2.

The malady that attacks the trunk of the tree is soon perceived, and agriculturists have been often alarmed at its progress and its destructive effects. It is a sort of ulcer which breaks forth almost always in the middle of the trunk; rare- 
ly on the north side, still more uncommonly on the east, but usually on the south, or the west side. When the moisture flowing from the ulcer increases sufficiently to reach, and actually attains the roots, the mulberry tree soon perishes. This disease is attributed to the setting of plants more than four years old ; for the stem becomes hard and dry in proportion to its age, and, taken from its native soil, at a more advanced age, is more affected by drought and heat, and thus losing part of its original moisture, receives the seed of future disease. This evil may be obviated by applying the knife, and taking out a circular piece of the bark, sufficiently distant from the diseased part, and cleaning the wood by scraping it where it is found necessary. A coat of bees wax and turpentine must immediately be spread over the incision, extending it a little beyond the edge of the wound, and, in two growing seasons, the cut will be closed, and the tree will recover its vigor and hardy vegetation.

Of the vermin which occasionally infest the mulberry trees, much might be said, but their enumeration would not be here of any use. Bonafous speaks of only two sorts, the Lamia Curculio, and the Lamia Lugubris, the grubs of which settle themselves beneath the bark and the ligneous or woody substance, and which 
ought to be carefully removed or destroyed. Pitaro speaks of the Scarabceus Melolontha of Linnæus, or the Melolontha of Fabricius. These insects, it appears, live several years under the earth, and the roots of the trees are so carefully gnawed by them, that although you take all the precaution to destroy these larvæ by all and every necessary means in your power, the mulberry trees are so damaged that they cannot live any length of time, and must die.

These attentions are of vital consequence; for a mulberry plantation contributes largely to the comforts of its proprietor, and when well managed, becomes to him a source of wealth. He will soon feel the all important difference between a healthy and vigorous tree, producing abundantly, and one that is sickly and yielding

a poor crop. 


\section{PA T I I.}

\section{CHAPTER I.}

OF THE REARING OF SILK WORMS.

Without going into the natural history of caterpillars generally, amongst which is comprised the silk worm; and as the rearing of silk worms must be in this country a source of industry to a class of people who more particularly need instruction on this subject, it has been thought proper to present at once, to that class, a condensed view of that mode of raising silk worms, which would best secure the reward of their labor.

The publications on this subject, in this country, appear to be either too voluminous, and too full of details, for the most part of minor importance, or too much condensed to contain the principal directions necessary to be attended to. This by no means pretends to ba e perfect or complete work. To endeavour to convey, in plain and perspicuous language, the practical knowledge which the author has acquired by experience, so as to enable the American farmers to undertake the 
rearing of silk worms, and the production of good saleable cocoons, with a reasonable hope of success, is his only aim.

The way to be certain of having good eggs of your own breed, will be hereafter shown, but if you are only beginning to raise silk worms, and are obliged to buy your eggs, you must, in a great measure, depend on the honesty of the seller, especially if you send for them to a distant country; the principal rule to be depended on in this case is, for the buyer to choose the small and bright seed, of a grey colour, more or less dark ; those that are white or yellow have been laid by the female without coupling with the male, at least have not been fecundated, and are of course good for nothing; and those that are brown, shrunken, and very flat are decayed. If you are near the place where the eggs were bred, you can best discover their size and goodness, before they are taken from the materials on which they were laid by the moth. Besides the choice of good eggs, some regard is to be had to the climate in which they were produced, so as to buy your eggs from a country which enjoys nearly the same degree of heat, or if any difference is to be made, they ought to be bought in a country a small degree colder than the one to which they are brought. 
Being provided with eggs, the next thing to be considered is the time of hatching them, and the best and surest rule for this is, to do it when you observe that the mulberry buds begin to open and spread into small leaves: and it will not be sufficient to observe this in one or two trees, which may chance to stand in a warm and sheltered situation, but it must appear through the bulk of all your trees, otherwise, though you may have a great number of them, you may be distressed for want of food.

Haring fixed upon your time of hatching, observe that all the directions which follow, concerning the manner of doing it should be attentively pursued; experience has demonstrated that the harvest of cocoons depends entirely upon well a managed hatching. 


\section{CHAPTER II。}

\section{METHOD OF HATCHING THE SILK WORM.}

ThE nature of silk worm's eggs is such, that, like those of other insects, they will, as the weather advances in warmth, hatch of themselves; but as, in this manner, there would be a great distance of time between those that hatch first, and those that hatch last, insomuch that, as some would every minute be hatching, it would be impossible to attend to collect them together, or afterwards to feed them, on account of their being sick at different times; therefore it is necessary to make use of a heat properly graduated, by means of stoves, in order that numbers may be hatched at one time, and that your whole quantity of eggs may come out at least in two or three days. You will keep separate the worms of each days' hatching, and if you can, you will collect them two or three times a day, and feed them by themselves; this is a thing so absolutely necessary, that, without it, there would be no possibility of feeding any quantity of silk worms to advantage, as will be shown hereafter. 
Various methods of making the eggs hatch, may be used according to the convenience of the breeders; but whatever method is used should aim principally at this end, viz: of making them hatch, as nearly as may be, together, on account of their moulting or casting off skin, and, as the only thing that will most effectually do this, is an equal degree of warmth, as near as can be attained, and constantly applied till they are hatched, a very simple and easy process to obtain such result, will be given.

The eggs are spread upon white paper, placed on a clean table, separating each ounce of eggs, and leaving a space of six or eight inches all round each parcel, for the reception of small leaves of the mulberry, which the silk worm eagerly seizes on as soon as it is hatched. This table, of a size proportioned to the quantity of eggs intended to be spread upon it, ought to be placed in a room seven or eight feet square, and seven or eight in height, closely wainscotted or plastered on all sides, having a glazed window on the east side, with a fire place, or (still better,) a stove, for the maintenance of a proper temperature. Three tiers of shelves, two feet long and eighteen inches wide, with a distance of two feet between the lower and the middle, and between the middle and upper tiers, must be fixed along the extent of two of the side walls. A fire must 
be made in the little room early in the morning, at noon, and at ten in the evening, for three days before the eggs be placed in it, in order that the air and the walls should be made dry and warm. The temperature of the air, during the first twenty-four hours after spreading the eggs, must be maintained at from 77 to $78^{\circ}$ of Fahrenheit, and each succeeding day the heat must be increased two degrees, till it shall arrive on the seventh dav, at 92 or 93 degrees, and never higher, when the worms will probably begin to make their appearance. This room should be furnished with two large dishes in which water may be poured, so as to offer a surface of four inches in diameter, at least; the moisture, which rises very slowly, moderates the dryness which might occur in the stove room. Very dry air is not favorable to the development of silk worms. Exposed to this graduated heat, the shell of the eggs on the sixth day appears much whiter. It is then that we must be unremitting in our care, turning the eggs every four or five hours, and preserving an equal temperature. Those which put forth before the seventh, or after the tenth day, are delicate and sickly, they should be set aside, and raised by themselves. The heat therefore, ought to be graduated with due reference to the climate of the region where the worms are bred, and the temperature in which they have 
been preserved during the winter. This is so true, and so worthy of remark, that if, in the winter the eggs have been kept in an atmosphere of 55 or $59^{\circ}$, or heaped together, they come forth without the aid of the stove, spontaneously, when the room is but slightly warmed, and before the mulberry tree has given any sign of vegetation. This circumstance is, therefore, of essential consequence, and should be noted to prevent its occurrence; to preserve the eggs in an even and gentle temperature, is therefore recommended.

The following are the signs of the speedy vivification of the silk worms:

The dull brownish slate color of the eggs grows bluish, then purplish, and then gray, with a cast of yellow, and finally of a dingy white, then the worm is already formed, and with a glass may be seen within the shell. To collect the worms, small twigs of mulberry, with a few leaves on them should be laid on the paper, and they should be increased as fast as the worms come out upon them. Care must be taken not to injure the worms with the small twigs by pressing too much upon them.

The worms which may have been managed according to the method here stated, will always be healthy and strong. They will neither be red nor black, but of a dark hazel or chesnut color, which is the proper color they should have. 
It is impossible to express the practical advantage of this method, thus ensuring the constant production of well constituted animals.

The appearance of the newly hatched worm is that of a woolly substance, of a dark chesnut hue, in which is perceptible a general stirring of minute animals, raising up their heads and presenting a black and shining speck or head. Their bodies are covered with regular lines of hair, or down, of various lengths. Their brown color is caused by this hair, the skin being whitish, which appears as they grow larger and the hair thinner. The whiteness of the skin is perceptible the moment the worm casts its shell and stretches its head. When seen through a glass, it appears to have a white collar; the tail is also bristled with hair.

When carefully following the precepts here given, there will invariably result the certainty of obtaining silk worms, healthy and strongly constituted.

More particular attention has been bestowed on this circumstance of hatching the eggs, as it is one of the most necessary points in rearing silk worms, yet, notwithstanding the many and minute things which are set down, the practice will be found sufficiently easy. The management of the worms when they begin to hatch, will next be considered. 


\section{CHAPTER III.}

\section{OF THE REARING OF SILK WORMS DURING}

THEIR FIRST AGES.

The worms, as they hatch, seek and cling to the mulberry leaves; they should be removed as soon as possible, and laid, with the twigs on which they are feeding, on wicker hurdles, covered with paper. In laying the twigs on the paper, care must be had to allow space enough for mulberry leaves to be put over the twigs, and between them, that the insects may have room to stretch and properly distribute themselves. It should be noted here, that the silk worms produced from one ounce of eggs, thus disposed, should occupy a space of nine and a half square feet, taking care to lay the worms in small squares of ten inches, which will exactly allow them the space they need until after their first moulting, but those who have the means of extending these allotted spaces may do so, because it is certain that the more room silk worms are allowed, the better they eat, digest, breathe and rest.

The first day after coming forth, and the distribution of the silk worms proceeding from one ounce of eggs, they should be given, in four 
meals, thirteen ounces of single, tender young leaves, chopped very small; dividing the time, so as to allow six hours between each meal, giving the smallest quantity for the first feeding, and gradually increasing that of each meal. Be careful that the leaves be always perfectly dry before you give them to the worms, which may be easily obtained by putting them under cover on a brick pavement, or gravelled floor, turning them over and placing them further where it is not damp, (for they always leave a dampness where they lie,) three or four times a day; you may thus keep leaves three or four days, provided there is no draught of air. Leaves cleansed and chopped very small are more suitable at this age. In this state they bite the leaf quickly, and it is consumed before it can be withered.

If care be not taken to chop the leaves small, and to give the young worms space when they are little, a great number must perish, as they will contract diseases, and lose their equality. 'The worm that cannot eat, dwindles, becomes extenuated, weak, sickly, and perishes under the leaf. This object, which appears trifling in itself, is however, of great importance, and deserves unceasing attention.

In feeding the worms regularly four times a day, you must manage so as never to give the 
whole quantity at once, as we stated above, because after the distribution of each meal, it is better to observe if some food should not be added in different spots. As they advance in this early age of their existence, if they consume their food readily and appear eager, it is well to give them a little food at intermediate times.

Thus, by much care, and all these appropriate and indispensable attentions, we lead this profitable little animal, at the end of five days, exclusive of the two days employed in its coming forth, and being removed and properly distributed, to its first dormant state, which results in the change of its skin. The signs by which it is known that worms are sick and about to change their skins, are these: they hold their heads up, are motionless, and appear to sleep; this should be noticed.

As it is from this age that depends the harvest of cocoons, it is then useful to wait the revivification of the greatest number of the silk worms before they are fed, particularly as these insects, when they cast their skins, need free air and gentle heat more than food.

In this first stage, the silk worms proceeding from one ounce of eggs, have consumed six pounds of picked leaves, well sorted and chopped very small, viz: 
lbs. ounces.

First day,

Second day,

Third day,

Fourth day,

Fifth day,
13

18

3

4

In this first age the temperature of the room should be kept at $75^{\circ}$ and the air be renewed only by opening the door; nothing further is necessary for the thriving of the worms, and their healthy continuance.

\section{Second age of the sitk worm.}

Fourteen feet eight inches square of table or wicker hurdles are needed for the accommodation of the worms, proceeding from one ounce of eggs, until the accomplishment of their second casting or moulting.

These tables or wicker hurdles, as before mentioned, should always be covered with strong paper. The temperature in this age should be about $73^{\circ}$. You must take care not to lift them from their litter until they are nearly all revived. There is no harm in waiting till they are all well awake and stirring, even should it be for twenty or thirty hours from the time when the few first began to revive.

When a great number of worms issue from the sheets of paper where they were placed, it 
is a sign that they should be removed from their litter, and removing them a little sooner, the others will soon revive also. There are always good opportunities of taking them up by means of twigs, to which they attach themselves. If the worms are laid on a newspaper, it is easy to take out the newspaper, lay it on a table, and transport the worms, which generally adhere to the leaves and branches, to another newspaper, which is put on the shelf after sweeping it. The litter on the former may then be thrown away. Instead of forming small squares, as was done for disposing of the newly hatched worms, long strips should be laid down the middle of the wicker hurdles, so prepared, that by widening them on each side when arrived at the consummation of the second age, the whole space of fourteen feet eight inches of the hurdle should be entirely covered by the silk worms. These means for removing the silk worms are the best, and suited to all the different ages, and the worms thus removed upon clean shelves with fresh boughs, get strong and revived.

An hour or two after the worms have been placed upon hurdles, they should be given a meal of one pound of leaves chopped small. In the remainder of this day you should give them $2 \mathrm{lb}$. $8 \mathrm{oz}$. of chopped leaves, in two meals, with an 
interval of six hours between each, or according to the hours of the day which remain. The second day they will require about $8 \mathrm{lb}$. of chopped leaves, in four meals, with intervals of six hours, the two first meals less plentiful than the remaining. It is very necessary gradually to widen on both sides the strips in which the worms are distributed, that at the close of this day two-thirds of the allotted space should be covered.

The body of the worm now acquires a clear hue; the head enlarges and becomes whiter. Should some places be thinly covered with worms, by placing small boughs where the worms lie thick, they will fasten on them, and may then be removed to fill up the places which were not sufficiently covered: the equality of the worms being very desirable, it should be carefully attended to, and those means above stated practiced through all the moultings, and whenever circumstances seem to require them.

The third day of this age they will require $8 \mathrm{lb}$. and $11 \mathrm{oz}$. of chopped leaves well picked, observing that the two first meals should be the largest. Now the leaves should be distributed in proportion as they are wanted, distributing them with attention, because the voracity of the silk worm abates towards evening; and many worms show, by raising up their 
heads, and not eating, that they are approaching the period of torpor, and some already are become torpid.

The strips should now be widened, so that at least four-fifths of the hurdle should be covered.

The fourth day they will require only $2 \mathrm{lb}$. $5 \mathrm{oz}$. of picked leaves distributed in the same manner as before, as may be wanted, lightly and carefully scattered over the worms.

This day the silk worms sink into torpor, so that the next day they will have cast their skins, and will be roused, and thus will the second age be accomplished.

The color of the worm is become of a light grey, the hair is hardly to be perceived by the naked eye, and is become shorter; the muzzle, which in the first age was very black, hard, and scaly, becomes immediately, upon moulting, white and soft, but afterwards again grows black, shining, and shelly as before, the length of its body is now rather more than six lines, and in four days its weight has increased fourfold.

As the insect grows, it breathes more freely, its excrements are more plentiful, which, as the number of hurdles also increases in the room, makes it necessary that the room should be better aired.

Should there be no wind or cold in the at- 
mosphere, the door and window may be left open for a little while. In this second age they have consumed $22 \mathrm{lb} .8 \mathrm{oz}$. of leaves, viz : first day, $3 \mathrm{lb} .8 \mathrm{oz}$.; second, $8 \mathrm{lb}$; third, $8 \mathrm{lb} .11 \mathrm{oz}$; fourth day, $2 \mathrm{lb} .5 \mathrm{oz}$.

\section{Third age of the Silk Worm.}

On the first day of this age, $3 \mathrm{lb}$. of the small shoots and $3 \frac{1}{2} \mathrm{lb}$. of picked leaves chopped rather coarser than formerly, will be necessary.

At this age the worms of one ounce of eggs should occupy nearly forty-six square feet of space, on the hurdles or tables which should always be covered with paper. The temperature may be now lowered and is not to be raised above $73^{\circ}$, but should it be higher externally, it may be checked by creating a current of air.

Particular care must be taken not to remove the worms from the wicker hurdles, until they have been all nearly roused from their torpid state; there would be no harm if those first revived should wait twenty-four hours, till the rest are all roused.

The following are the most certain signs that the worms are roused: They issue from their old skin with so different an aspect, that any one may distinguish them without the aid of description; they make an undulating motion 
with their heads when horizontally blown upon, which is a never-failing sign that they are roused, then a gentle motion of the air through the room does them good, provided the renewed air is not colder than their usual atmosphere.

You should allow to the worms in this third age forty-six fuet square of space, as has already been observed, disposed in a strip down the centre of the wicker hurdle, so as to leave rather more than a quarter's width down each side of the strip. The space to be occupied by the silk worms, in their different ages, being well ascertained, there will be nothing more easy, more useful, and more economical than to remove, cleanse, and place them in the manner above described.

Until their casting is accomplished, you need not touch the worms. Once plased upon the wicker hurdles, they feed well without interfering with one another, and without requiring to cleanse the intervals on the sheet of paper. 'Their litter does not become mouldy, unless there should be a very unusual and continued dampness of weather, but is of a fresh green color, thinly scattered, nearly dry, and composed of the fibres of the leaf, and little portions of the leaf fallen from the mouth of the animal. 
The $3 \mathrm{lb}$. of young shoots afford the silk worms their first meal, as in the preceding age; but when they have eaten the leaves upon the shoots, they should have a second meal of about $1 \frac{3}{4} \mathrm{lb}$. of leaves, carefully filling with them the space between the shoots, in order to equalize the distribution of the worms upon strips; the last meal to be given this day will be of $1 \frac{3}{4} \mathrm{lb}$. again of leaves.

At the end of the second or the third meals, there will be a very sensible change in the silk worms. They are much larger, their muzzle is grown longer, and their color clearer.

On the second day they will require $17 \mathrm{lb}$. of picked and chopped leaves, the smallest quantity given during the two first meals, because, near the end of the day, they grow voraciously hungry. Care must be taken to widen the strips when they are fed, in order to allow them room.

The third day they will eat $19 \frac{1}{2} \mathrm{lb}$. of picked leaves, chopped, and divided in four meals, the two first being the most plentiful. Towards evening their hunger decreases, consequently, the last should be the least meal.

This day the silk worms grow fast, their skins whiten, the bodies are nearly transparent, and the heads are longer.

The contortions they begin to make with their 
heads, show that their change approaches.

The fourth day they will eat $10 \frac{\mathrm{I}}{2} \mathrm{lb}$. only, of chopped leaves. The decrease of food is consequent upon the diminution of appetite already mentioned; many of the worms are already torpid.

They should be given four meals, the largest first, and the last the least, those only that seem to require it, should be fed. Should a great number of silk worms on one table be torpid, whilst others continue to require food, you must not wait for the regular hours of their feeding, but give them a slight meal one or two hours after, in order to satisfy them, and that they may sink quickly into torpor; this care is of consequence, and these intermediate meals are very beneficial.

On the fifth day of this age the worms will require $5 \frac{\mathrm{I}}{2} \mathrm{lb}$. of picked leaves, chopped, and distributed as they are needed, this will be about the quantity, if it is not enough, more may be added, if too much, less can be given.

These two last days the silk worms begin to cast about some silk down. The insect seeks free space to slumber in, dry and solitary spots, rearing its head upwards, which is known by finding it on the edges of the paper, where any stalks stick up, upon which it retires. All of them not being able thus to separate from each 
other, are obliged to remain upon their litter, but testify uneasiness by rearing up their heads.

When on the point of sinking into torpor, they completely void all excremental matter, and there remains in their intestinal tube, a yellowish lymph alone; rather transparent, and which supplies almost all the animal fluid in them. It is that which, before the surface of the skin they are going to cast, becomes wrinkled and dry, causes them to appear of a yellowish white color like amber, and semi-transparent.

When the worms prepare for the third, and even fourth moulting, the air of the laboratory should be gently agitated, but the temperature should not be much varied.

On the sixth day the silk worms begin to rouse, and thus accomplish their third age.

The general view of this age presents the following results.

1st. They have consumed nearly sixty pounds of leaves and young shoots.

2d. Their average length, which was six lines after the second moulting, has become, in less than seven days, above twelve lines.

3d. Their weight has increased fourfold in the same period, and their bodies are more wrinkled, particularly about the head, having no appearance of hairiness. 
It has been sufficient during this age to open the door and the windows, when the weather was still and fine, so as to lower the temperature by a degree only. 


\section{CHAPTER IV。}

\section{REARING OF THE SILK WORMS IN THE}

FOURTH AGE.

Your worms being recovered from their third moulting, will be pretty large, as we have already said, they should, therefore, occupy a space of 109 square feet, and having now arrived to a state of vigor, the temperature of the room should be diminished, by keeping constantly a draught of air, and if the temperature should be rising from $z 1$ to $80^{\circ}$, and the air stagnant, it is recommended to burn some faggot wood in the fire-place, which should be, if possible, situated in an angle of the room, to create a complete current of air, and thus change the air of all the room thoroughly. If, instead of thus acting, when the heat of the season rises suddenly, (which augments the fermentation of the litter,) we should exclude the exterior air from the room, we may expose ourselves to lose the whole brood, because, as the worms grow, the mass of leaves and litter increasing, the dampness proceeding from it will more quickly produce fermentation, the heat 
would also increase, and the air would soon be not only damp, but pestilential.

As has already been said, you should not remove the silk worms, from the hurdles on which they have accomplished their third age, until they are nearly all well roused, because, though the first roused should remain a day or a day and a half before being removed, it will not hurt them; you will put the early roused in the coolest part of the room, and the hurdles of the late roused in the warmest part. If you do not wish to take so much trouble, it may suffice to give the latest roused worms more space, by keeping them farther asunder; by proceeding thus, they will soon come up to the others.

It will be easy to ascertain by the thermometer, which parts of the room are constantly the hottest; this knowledge will serve to render all the silk worms even sized, particularly if those who attend them have any practical skill. Those precautions are indispensable if you wish the worms to begin to spin at the same period, inasmuch as there arises great evils, when some of the silk worms begin too long before the others.

We must here repeat how advantageous to the art of rearing silk worms is the practice of distributing them in regular strips and squares which are filled with these insects gradually, 
and only when they have accomplished their various ages.

1st. Because you need not clean the hurdles in the fourth age, the litter which spreads by degrees, not heating or contracting any effluvia, and not rising much.

2d. Because the leaf, distributed upon evenly portioned spaces is entirely eaten before it is withered and spoilt.

3d. Because, by this practice the worms can feed with facility, move with ease, perspire and breathe more freely; all decisive advantages for these insects.

These advantages cannot be obtained when the worms lie too thick; in that condition they cover the surface too closely, the leaves on which they lie are wasted, as they cannot possibly eat them; while, on the contrary, when they have plenty of room, they seek, in moving, every atom of the leaf and eat it up. Besides, when straightened, the action of their breathing and perspiring tubes is hindered and confined by the pressure, either superior or lateral, of one worm against the other; whereas, if they have full space, the action of these organs, which is so necessary to their health, is very free.

The first day of this age they will require about $7 \mathrm{lb}$. of shoots with leaves, and $12 \mathrm{lb}$. of picked leaves coarsely chopped. 
When the moment for removing the worms from the hurdles comes, one or two hurdles only at a time should be covered over with young shoots, which, when loaded with worms are removed, as in the first moultings; but, as in this age the worms are heavier, it is recommended to put the shoots loaded with them upon some portable trays; thus the worms will be better removed, and carried with less inconvenience.

The strips on which the worms are laid upon hurdles, should occupy about half the space allowed them, or $54 \frac{1}{2}$ square feet. After having removed all the early roused, if they have eaten all the leaves on the shoots, and should remain without food upon the hurdles, you will give them $6 \mathrm{lb}$. of leaves chopped a little; with these leaves the intervals between the young shoots should be filled, and form the strips into regular order, by sweeping into their place any boughs or leaves that are scattered irregularly, with a broom made for that purpose; after this second meal, the worms formerly heaped up together, will be seen stretching out evenly.

The other $6 \mathrm{lb}$. of leaves should not be given until the second meal has been entirely consumed.

Although it is not the general custom to chop 
or cut the leaves for silk worms in this fourth age, it has been, however, found very beneficial to give them, coarsely cut up, not only the first day, but also the second and third. The reason is, that when the silk worms are just issuing from the cast skins, they are weak, and not very hungry; therefore fresh leaves, slightly cut up, by exhaling a stronger smell, stimulate their hunger, and the cut edges are more easy to bite.

The late roused silk worms should be placed on hurdles distinct from the earliest.

At the end of this day, the worms begin to show some vigor; they move quickly to the leaves, they grow perceptibly, tiney lose their ugly colors, become slightly white, unless they are of that -spotted species which the French call tigrés, and assume more decided animal action.

The second day they will consume $33 \mathrm{lb}$. of sorted leaves, slightly cut up. The two first meals should be the lightest, and the last the most copious. In giving the meals, the space occupied by the worms should be widened.

The third day there will be needed $45 \mathrm{lb}$. of sorted leaves, a little cut. The two first meals ought to be the most plentiful, the last meal the lightest.

The fourth day you should give them $51 \mathrm{lb}$. 
of cut leaves, the three first meals should be the most copious, and the fourth the lightest. The worms become still whiter, and at this period are more than an inch and a half long.

No more than $26 \mathrm{lb}$. of picked leaves will be needed the fifth day, because the hunger of the insect diminishes much; the first meal should be the most copious. A great number of the worms become torpid on this day. You will distribute the leaves as they will be wanted, and only on the hurdles where the worms are perceived not to be torpid; they will be this day an inch and three quarters long.

The sixth day seven pounds of picked leaves will be enough. It is easy to see where and in what quantities the worms need food.

Since the preceding day the silk worms begin to decrease in size, as they have cleansed and cleared themselves of all nutritive substances, before they sink into their torpor. The greenish color of the rings has disappeared, and their skin seems quite wrinkled.

On the seventh day of this age, they rouse, cast their skins, and accomplish their fourth age, having consumed, in that period, $173 \mathrm{lb}$. of sorted leaves, and $z \mathrm{lb}$. of shoots with leaves, and occupied 109 square feet of space, gradually allowed to them.

On that day the worms, which were before 
about one inch long, have grown half an inch in length, and their weight has augmented four fold.

In order to renew and lighten the air of the room, without particularly heating it, dry straw or shavings of wood should be burnt in the fireplaces, three or four times a day, having, at the same time, the door and windows opened for the circulation of air; and if the exterior temperature be not cold, and without wind, they may remain so, 'till the interior temperature is lowered half a degree; thus the silk worms will breathe continually a pure and dry air, which makes them most healthy. 


\section{CHAPTER V.}

\section{OF THE FIFTH AGE-FIRST PERIOD.}

Tius period embraces the management of the silk worms from their recovery out of their fourth moulting, until they are ready to spin their cocoons.

As the fifth age of the silk worms is the 'longest, and most decisive, a few practical observations will be given, before resuming the description of their daily progress in this age. It is not intended here to give scientific lectures, but to endeavor to make some truths obvious, which the intelligent cultivator may easily put into practice, to protect himself in all cases from those losses to which even a man of experience may be exposed, not knowing those truths.

Should the worms die in the first age, the loss is trifling, because the expense is not prolonged, while, on the contrary, should they perish in the fifth age, the loss is considerable. It is, therefore, very important to know the condition of the worms in this age, to learn how to manage them, so as to insure their health and strength against the effects of the atmosphere or other evils that may assail them. 
It is mostly in this age that you will find the advantage of keeping the worms on hurdles; for as they will now eat during nine or ten days before they are ready to spin, and will grow very large, making a prodigious deal of litter, they would, if they were kept upon shelves made of boards, require to be cleaned every day, which would be a considerable trouble; but on hurdles made of small reeds which are very dry and light materials, and have vacant spaces between them, the air will have free access to the litter which sticks and remains among the fibres and shreds of the leaves, and it will not so suddenly become putrid, moist, and mouldy, as it would do if the whole quantity of litter and fibres should lie on boards or shelves, which did not admit the air underneath.

Any one will appreciate the above recommendation, particularly when he knows that as the silk worms grow in the fifth age, they are liable to three evils, which injure them according to their strength, and to their distribution in the room, and may weaken them so as to cause their speedy destruction.

Those enemies are:-

1st. The incredible quantity of fluid disengaged every day from their bodies by the transpiration and evaporation of the leaves given to them. 2d. The deadly and mephitic emanations 
emitted every day from the insects, from their excrements, from the leaves and their remains.

$3 \mathrm{~d}$. The damp hot atmospheric air, as well as the smothering heat of the cocoonery during the fifth age.

These evils injure the silk worms in three ways:

1st. The moist exhalations produced by the leaves and the perspiration of the insect, accumulate in the room, and tend to relax the skin of the worms; this organ thus loses its elasticity, puts the animal into a state of languor, decreases its appetite, alters its secretions, and makes it liable to various diseases, and even to death.

2d. The mephitic emanations issuing from the body of the insect, and from the leaves, render the silk worm's breathing difficult, destroy its excitability, and produce disease and death.

3d. The dampness and stagnation of atmospheric air, increased by the moisture of the room, create a great fermentation in the dung, and consequently, disengagement of heat, which, by destroying the elasticity of the air, renders it so deadly, as, in the course of a few hours, to destroy the silk worms entirely.

"To these causes of sudden disorders we often have to add another, which proceeds from the silk worms being too closely distributed on the hurdles, particularly in this age. This in- 
sect, as people generally seem to think, does not breathe by the mouth, but by small apertures, which are placed near its legs, and which are called stigmata. These breathing vessels are almost all stopped up and covered when the worms are heaped together, which makes their breathing difficult, and the perspiration also ceases, much to the detriment of the insect.

It will be easy to avoid the above diseases, 1st, by evenly distributing the worms in spaces proportioned to their numbers, as has been frequently repeated; $2 \mathrm{~d}$, by renewing the air, and keeping the cocooneries dry and lightsome by the means already indicated; $3 \mathrm{~d}$, by taking care to gather the leaves some time before they are wanted, and when they are wet, to have them thoroughly dried before they are given to the worms; 4 th, by keeping the hurdles on which the worms are placed free of all excremental matter; 5th, and lastly, by avoiding or preventing smoke, which may occasion the instant suffocation and death of the silk worms of a cocoonery, particularly should there be any dampness in it, which is unluckily too often the case.

These few observations containing all the instructions needed to put any one on his guard, it is now time to bring the worms to the period when they prepare to rise, and when they re. 9* 
ject the food they had lately so voraciously devoured.

The silk worm comes out from its last moulting with a larger head and body, the first wrinkled and the other rather shrunken; the room should have uniformly 68 or $69 \frac{1}{2}^{\circ}$ of heat, and at the termination of their fifth age, the silk worms should occupy 239 square feet of hurdles. They will consume in this age $1098 \mathrm{lb}$. of sorted picked leaves.

On the first day of this age the worms should fill a space of about $99 \frac{1}{2}$ square feet of hurdles, they will eat a prodigious quantity of leaves, which should now be of the best sort you can procure for them, because it is now that they collect and digest the matter out of which they afterwards form their silk. If you were to open a worm before the fourth moulting, you would find nothing but a watery humour mixed with the green mucilage of the leaves which they feed on ; but when they are somewhat advanced in this last age, you will find in their silk vessels a pale or yellow gum, out of which they form their cocoons.

The goodness, therefore, - of their silk depending in a great measure upon their being properly fed at this time, care must be taken that their food be, in all respects, as good as can be got; not the soft, tender leaves of shoots 
furnished them with an abundant meal; and you should now divide eighteen pounds of leaves more into two meals, which should be given them at the interval of six hours. In giving the first meal, care must be taken to regulate the strips on the hurdles, by sweeping any straggling leaves or worms into regular order with the little broom.

At the third meal, that is to say, when you distribute to the worms the remaining nine pounds, you may widen again the strings a little more, and should there be too many worms in some parts, they should be removed instantly, and placed on the least crowded parts of the hurdles.

This day the worms appear tolerably strong.

Attend to the temperature, which, if too high might exhaust their vigor, and diminish the quantity of silk. With rare exceptions the season now calls often for a pure, cool, and refreshing air to relieve and comfort all kinds of animated beings. Silk culturists agree, that if the dryness of the atmosphere should be such as to affect our own feelings, the floor of the cocoonery should be sprinkled with water, and buckets of water be kept there, which will afford a salutary moisture by exhalation. Let us not, however, expose the worms to an opposite extreme, which would, no doubt, retard their 
last urgent task, or prevent them from filling well their cocoons.

The second day the worms will consume fifty-four pounds of sorted leaves, divided into four meals; the first should be the least, and the last the most plentiful.

In distributing the food the strips should be widened gradually; at the close of this day the worms are much whiter, and considerably developed.

The third day they will require about eightyfour pounds of sorted leaves. The first feed should be about fifteen pounds, the second and third of twenty each, and the last of about thirty pounds. They continue to whiten and many appear upwards of two inches long; they could eat, on this day a larger quantity than here specified; but it is most beneficial not to add to this quantity, that they may thoroughly digest it; besides which, it strengthens their constitution, and makes them livelier. The strips they occupy should be widened whenever they are fed.

The fourth day they will want one hundred and eight pounds of sorted leaves, the first feed should be the smallest and the last the largest; their voraciousness seems to increase; they grow larger and stronger; some are two inches and a half long.

The fifth day they will consume one hundred 
and sixty-two pounds of picked leaves, you will distribute to them in the same proportions as the preceeding day, the last meal always the most copious, and if necessary you must not confine them to four meals, but must take care that they get some intermediate food; when the regular distribution of leaves is devoured in less than an hour and a half, the worms must not be suffered to fast four hours, but have some leaves in the interim, particularly if there should be any hurdles on which the worms had not been at first as well fed as the others; for although the quantity of food has been fixed for this day, it is always necessary to be regulated by experience.

In the course of this day the hurdles should be cleaned. If the litter is dry and fresh, the worms should not be shifted till the evening, or the beginning of the next day. The following manner is employed to clean the worms at this period.

You will place a line of fresh leaves the whole length of the hurdles near the worms, to which they will immediately attach themselves; and as soon as they get on the new leaves, the litter they have left can be swept away, and removed from the room. During this operation, if the exterior air be damp, which would indicate that of the laboratory being still more so, there should be burnt light blazing fires, and if this 
should raise the temperature too much, it may easily be lowered by openning the doors and windows.

On the sixth day the silk worms should have $195 \mathrm{Ib}$. of picked leaves, which you will divide in five meals, the last of which should be the most plentiful. The worms now eat most voraciously, and some even attack the mulberries which are amongst the leaves.

If, after having distributed the leaves, the quantity appears insufficient upon some hurdles, or if it has all been devoured in an hour, you should add an intermediate meal.

Knowing the quantity of leaves to be given in this day, it is easy to distribute them either into four or five meals, as may best appear to suit the worms.

The shelly black shining proboscis placed at the extremity of the muzzle is become stronger; it is in this proboscis that are placed the small saws which tear and separate the hard leaves, and even the fibrous parts of the leaf.

Some of the worms are now three inches long, they are whiter, they present to the touch a soft velvety surface, and are strong and healthy. Take care to give more food to those which have been last removed, and allow them more space, and they will soon equal the earliest in size.

They will require on the seventh day, one 
hundred and eighty pounds of well sorted leaves. The first meal should be the largest, and the others should diminish; should there be any intermediate meals wanted, they must be given as before.

- Some worms will now be seen upwards of three inches long, the extremity of the insect begins to grow shining and yellowish, which shows they are approaching to maturity. Some of them begin to eat with less voracity. They have now attained their utmost growth, being upwards of three inches long, and an inch and a half round, nearly.

Towards the close of this day they begin to lose size and weight, because from this time they take less food in proportion to the quantity of excrement and steamy vapor which their bodies discharge.

We shall continue to observe them as they decrease, as we have done while they were increasing. They are at present in their highest condition.

They must have on the eighth day, one hundred and thirty-two pounds of well sorted leaves. The proportion of leaves must diminish, as the appetite of the worms decreases much. The leaves, as usual, should be divided into four meals, the first being the largest, and gradually diminishing.

In order to bring on backward worms to ma- 
turity, some intermediate food should be given, according to their wants.

They now advance towards maturity, which may be perceived by their yellow color, which increases from ring to ring. Their backs begin to shine, and the rings lose the dark green color that marked them. The advance to maturity is also denoted, in some of them, by the diminution of their bulk in the course of this day; and by their trying to fix themselves to the edges of the hurdles, to void the substance with which they are filled.

This day, and as speedily as possible, according as the signs of maturity increase, and as the litter gets moist, the hurdles should be cleaned in the manner before described, being very careful not to bruise the worms. It is now that light fires, and the purification of air are more essential than on any former change, particularly in a large laboratory.

On the ninth day distribute to the worms ninety-nine pounds of leaves, as may be wanted. On this day their yellow hue grows deeper, their bo dies will be of an ivory color and polish, somewhat transparent towards their necks with a little of a golden appearance. "The muzzle is become of a brighter red than it was in the beginning. From time to time a gentle fire should be lighted, particularly in the night, and the air entirely renewed, 
Most of the worms will leave off eating, and before they are ready to spin, they will somewhat lessen in size; but their bodies will feel more firm and consistent than before.

They will begin to desist from eating, and to wander about, stretching out their heads in quest of a place proper to fix in and spin their cocoons. Now although, on account of preserving some little order and method, the instructions for accommodating them at their spinning time, are not given till the next chapter, yet it is necessary that you should have looked into that part, in order to have made the suitable preparations for them some time beforehand; for you would find yourself greatly embarrassed to do it when numbers of the worms were to be accommodated, if you had not made some provision before.

It may be proper, before we end this chapter, to insert here a table of the rearing of silk worms from one ounce of eggs, taken from the work of M. Bonafous, a distinguished pupil of Count Dandolo. The only difference between the pupil and his great master is in the spaces prescribed by Monsieur Bonafous, which are greater than those marked by Count Dandolo.

We must here inform our readers that the measures and weights in the following table, as well as those which we have made use of in the 
progress of our own work, are French, the first, when reduced to American measure, are as follow, fractions omitted:

French Feet. Inches. Am. Feet.

\begin{tabular}{|c|c|}
\hline 9 & 6 \\
\hline 19 & \\
\hline 46 & \\
\hline 109 & \\
\hline 239 & \\
\hline
\end{tabular}

And the quantity of leaves when reduced to American weights, are as follow, omitting fractions :

$\begin{array}{rrr}\text { French lbs. } & \text { Am. lbs. } & \text { oz. } \\ 7 & 7 & 12 \\ 21 & 23 & 4 \\ 69 & 76 & 9 \\ 210 & 229 & 8 \\ 1,281 & 1,400 & \end{array}$

The French foot is divided into twelve inches, and the inch into twelve lines. It is longer by nine lines than the American foot, or one foot 7.100 American, nearly. Those who intend to regulate the spaces by the rules of either Dandolo, (which we have followed, ) or by those of Bonafous, may easily do so, by noting the dimensions of each hurdle or feeding frame. The dimensions of Count Dandolo may be considered as a minimum, or the least that can be allowed, to secure the health of silk worms. It cannot be too often repeated, that the constant rule to be attended to, is to give the worms ample space, and never to permit them to be crowded. 
Table of the rearing of silk worms to

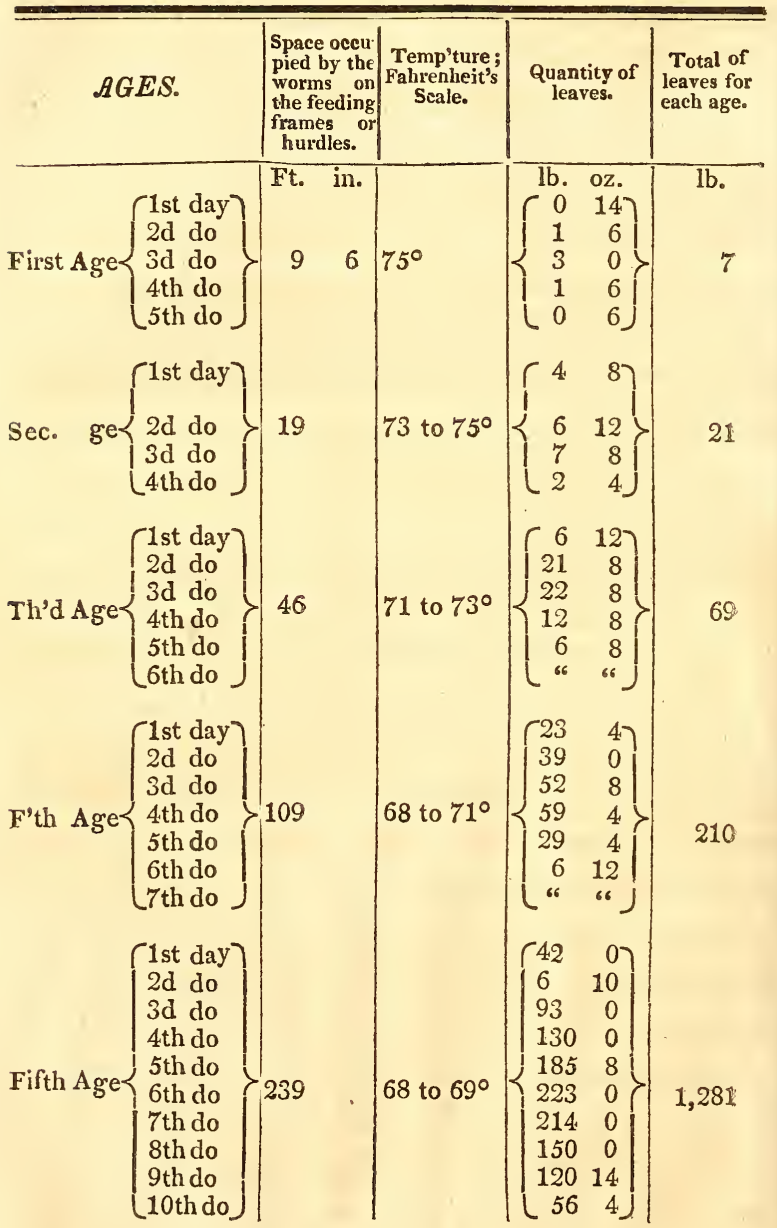




\section{the sixth age, from one ounce of eggs.}

\section{OBSERVATIONS.}

Tender young leaves chopped fine; four meals, progressively increased.
do
do do
the first the largest, the last the smallest.
do
do do
four meals.

The first meal of $9 \mathrm{oz}$. , the others less, if the leaves have not been eaten.

Tender leaves, chopped fine. Worms casting their first skins.

Half tender branehes and half leaves, cut fine; the first meal twelve ounces, the other leaves in two meals.

Tender leaves, four meals; the two first less than the two last. Enlarge the spaces. do do the two first the largest.

do do distributed as wanted. Worms casting their second skins.

Half tender branches, half leaves a little chopped; second meal $1 \mathrm{lb} .14 \mathrm{oz}$.

Chopped leaves four meals; the two first less than the two last. Enlarge the spaces.

$\begin{array}{llll}\text { do } & \text { do the two first the largest. } \\ \text { do } & \text { do } & \text { do } & \text { do the last, least. } \\ \text { do } & \text { do distributed as required. The third casting of their skins. } \\ \text { do } & \text { do } & \text { do } & \text { do Worms are roused. }\end{array}$

$9 \mathrm{lb}$. of branches, $14 \mathrm{lb}$. of leaves, cut coarsely; give the branches first.

Leaves coarsely cut, four meals; the twe first the smallest. Enlarge the spaces.

do

do three meals; the three first the least, the last $17 \mathrm{lb} .4 \mathrm{or}$.

Whole leaves, four meals; the three first $16 \mathrm{lb} .4 \mathrm{oz}$. the last $10 \mathrm{l-2} \mathrm{lb}$.

Picked leaves distributed as wanted; the first meal the largest.

do do do do Worms prepared to cast their skins.

Leares as wanted. Worms are roused.

Half branches half leaves. Enlarge the spaces.

Picked leaves, four meals ; the first the smallest. $12 \mathrm{lb}$. the last $22 \mathrm{lb}$.

do the first meal $22 \mathrm{lb}$. the last $27 \mathrm{lb} .12 \mathrm{oz}$.

do do $27 \mathrm{lb}, 12 \mathrm{oz}$. the last $371-2 \mathrm{lb}$.

do do $371-2 \mathrm{lb}$. the second $46 \mathrm{lb} .14 \mathrm{oz}$.

do fuur meals, the last the most abundant.

do do the first meal the largest, the rest to lessen gradualiy.

do four or five meals, the first the largest, $46 \mathrm{lb} .14 \mathrm{oz}$.

do distributed as wanted. Worms approaching maturity.

do do do if not enough give more. 
The general result of what we have stated is that in the course of about thirty days, in which the silk worms have attained their greatest development and heaviest weight, we may observe the following facts:-

1st. That in their growth they have become forty times larger than they were when first hatched, being then about the size of one line.

2d. That in thirty days their weight has become nine thousand times greater, since it required 54,525 young hatched silk worms to form an ounce, (vide Dandolo, chap. v, §3,) whereas six silk worms, when full grown, are now sufficient.

From the ninth day of the fifth age, and the thirty-first of the life of the silk worm, until the completion of its maturity, we shall see, that although they need little food, they will require great care, of which we shall speak in the following chapter. 


\section{CHA P TER VI.}

SECOND PERIOD OF THE FIFTH AGE-THE SPINNING OF THE COCOONS.

Let us for a while leave the silk worms on the hurdles, to mention some things relative to them, and to show the preparations we should make for the accomplishment of their fifth, and in the shape of worms, their last age.

This age can only be looked on as terminated when the cocoon is perfect. When the worm has poured out all its silk, and formed its cocoon, it casts its envelope, and becomes a chrysalis. But to form the cocoon, it must attain to this point, viz: of becoming a compound of only two remaining substances, the silky substance and the animal substance. It must then have discharged all the excremental matter contained in its intestinal tube.

It is not only requisite to know the last degree of perfection of the worm in order to facilitate the means of forming its cocoons, but also to know all the other operations necessary to insure the cocoons being of a very good quality.

The cleanliness of the hurdles in these last 
days of the fifth age, requires great attention to preserve the health of the silk worms.

It is with these insects as with all other animals, some are quick in all their operations, others more slow; and it is important to form just observations on these facts.

We have seen, in the foregoing chapters, how the worms begin and continue to show signs of maturity. This last day they attain perfection, which may be ascertained by the following indications: They crawl on the leaves without eating them, they rear their heads as if in search of something to climb on; their rings draw in; the skin of their necks becomes wrinkled, and their bodies have more softness to the touch than heretofore, and feel like soft dough. Their color also changes, and the whole body assumes the transparency of a ripe yellow plum. When these signs appear in any of the insects, every thing should lie prepared for their rising, that those worms that are ready to rise may not lose their strength and silk, in seeking for the support they require.

We have now brought the worms to that period of their lives, at which your labor of feeding them ends, for, from the time they are quite ready to spin, they take no more food of any kind, though their lives in different forms last near a month longer, and in cold climates a good deal 
more. Yet, though you will be eased of the trouble of feeding those which are come to their spinning time, you must, for a few days, till they are all settled in their work, give them a diligent attention.

To avoid the loss that might accrue from delay, there should be bunches ready made of small twigs, or common broom, or of clean bean stalks, or, in short, of any bush, or brush wood, free from leaves, dirt, moisture, mouldiness, or offensive smell. With these twigs you are to form several long arbours, arched at top, quite across each shelf; the distance between the sides of these arbours, should leave the arch open quite through, so that there may be room to put in your arm, and feed those worms which are not yet quite ready to spin.

You should form these arbours so as to have a sort of bushy appearance without being too thick or too thin. But if the making of them be thought too troublesome, it may sufficiently answer the same purpose to place between the shelves branches of broom a little aslant, so that the worms that climb up may run no chance of dropping off, taking care to spread out the branches like fans, that the air may penetrate through all parts, and the worms may work with ease. When the worms are too near each 
other they do not work so well, and form double cocoons, whose silk is of an inferior quality.

It would be endless to give all the methods which might be contrived for this purpose, but whatever mode may be adopted, it is always desirable that the bushes should be well placed, well arched, clean and light, and not too thick; that as we have already said, the air may circulate freely, and that the worms may work with ease.

While the silk worms are seeking places to spin their cocoons in, some of them will often wander about the middle of the hurdles, wasting their silk in useless floss. Indeed, if the hurdles are furnished with arched arbours, as recommended, the silk worms will scarcely miss a place where they may readily spin their cocoons; but in large hurdles, that have the twigs only placed round them, you must often look, and whatever worms you find wandering under the hurdles, or far from the arched arbours, you must take and place them near the twigs, provided they are ready to spin, and their wandering from their food is a sign that they are so; you need not fear hurting them by taking them in your hands; but observe to take them up with whatever sticks to their claws, without tearing it away, for fear of blunting the claws by which they are now to climb. 
As many of the worms will continue to eat for some time after others have begun to spin, you must constantly supply them with the best kind of leaves, sprinkling them very thinly over them, and feeding them often, and this even at night just before you go to bed, and as early as you can in the morning; for their quantity of silk, and their strength and activity in spinning it, depends now upon their being properly and fully supplied as long as they continue to eat; for which reason, you must give them leaves often, but few at a time, because if they lie under the arbours it is not easy to clear away their litter, which, however, must be done.

This operation, although tedious, is easy enough, with the aid of the portable trays; and by carefully putting the silk worms upon them. This done, the litter should be cleansed and the worm gently slided down upon it by slanting the tray. They should, strictly, only be given the quantity of food they may want, and that very sparingly.

In sliding the silk worms upon hurdles, they should be placed in squares of about two feet, beginning on the side upon which the arched arbours are already placed, and forming the squares close to them, so that the silk worms 
may find no difficulty in rising upon the bushes; a distance of eight or ten inches must be left between the squares. In the centre of these squares should be fixed bushes of broom, or small dry boughs, to afford the worms room to spin, and not leave the vacuities amongst the branches too large, because they would waste a great deal of their silk, before they can fix on a proper place; the size of their cocoons, which is that of pigeons' eggs, will direct you in this matter.

If you find that as many worms have got amongst the branches as can conveniently spin there, then take away those which have not yet mounted, and place them upon another hurdle, with worms which will spin nearly at the same time; for if the worms are too much crowded in the arbours, they will be more apt to spin double cocoons, which having two worms in one, cannot be easily reeled off: therefore, as you look over the arbours, wherever you see two worms begin one common cocoon, take one of them away, and place it in some other part of the twigs.

A little before the worms on any stand are ready to spin, you must clean away their litter from all the hurdles, that the stands may be sweet and airy at the time of spinning, and that 
you may not at that time disturb or shake the arbours where they are spinning; for this would stop and interrupt their work, so that some would desist from making any more silk and the cocoons of others would be ill-formed, and difficult to reel off; therefore you must take care to shake or molest them as little as you can.

As you were directed, in feeding the worms, to keep those of the same age on the same hurdle, so you should also in distributing them as they grow large, always keep them on the same stands, that they may spin at the same time. It is supposed that you have many stands of hurdles.

When most of the worms on any hurdles have climbed the branches in order to spin their cocoons, you will generally find some on each hurdle, which are weak and lazy, which do not eat, and do not seem of the disposition of those that have risen, but remain motionless on the leaves, without giving any sign of rising; these should be collected from all the several hurdles where they lie, and should be put in a dry clean room of at least $73^{\circ}$ of heat, where there are twigs or branches prepared for them.

As soon as they are thus placed, some will rise directly, others will eat, and then rise, and so on until all shall have risen. These worms 
will soon acquire the vigour and stimulus they want, by being put in a warmer and much drier a partment.

Cold and damp weather during the time of their spinning is extremely hurtful; in very cold weather the worms desist from their work; you may see them, while their cocoons are yet thin, either moving very slowly, or quite inactive; if you remove one of the cocoons to a warm place, the worm immediately begins to work with activity, and desists again when put in the cold, where, if it continues, the worm at length totally leaves off its spinning, and is changed into a beetle or chrysalis, which is its state in the cocoon before it becomes a butterfly. This interruption, though the worm should resume its spinning, makes the cocoon difficult to wind off, the thread often breaking; therefore, if this kind of weather should happen, you must make fire in the room, and if the temperature becomes too high, admit fresh air for a little while.

This inconvenience of cold and moisture will happen chiefly in cold and changeable climates; but in hot climates you will oftener have occasion to guard against sultry and suffocating heat, which can easily be done by leaving the current of air free on the side where it blows coolest. 
In large laboratories the great mass of silk worms in evacuating themselves, often soil one another with excremental matter. Moisture, as we have frequently observed, is very pernicious to these insects, and, if once wet, it checks their transpiration: that alone will destroy their vigour, and indispose them to rise. As fast as the hedges and clumps are formed, the worms that rise on them shed a liquid matter upon the litter and paper, where lie the later silk worms, which increases their languor and listlessness, even supposing the air to be pure; if it were damp, they would be slower and lazier still, so the best is to remove them at once to a tolerably warm spot, provided with branches and a few fresh leaves.

With this assistance, the lazy worms will distribute themselves in the branches, evacuate, and begin to spin their cocoons.

As soon as all the worms are off the hurdles, or have either risen, or been carried away, no time should be lost in cleaning the hurdles, which will be the last time of performing this operation. It is beneficial to carry off, as quickly as possible, every thing that tends to corrupt the air, or make it damp.

The fifth age is accomplished when the silk worm pours out its silk, and forms the cocoon. 
The cocoon is perfected when it has attained a firm consistence, which is known by pressing it gently with the fingers, the silk worm then casts its envelope and has become a chrysalis; here ends its fifth and last age. The foreign writers reckon as many as seven ages ending with the dropping of the eggs by the butterfly; as naturalists, they may be right; as agriculturists we think this division unnecessary, and reckon only the ages of the worm while it continues such. 


\section{CHAPTERVII。}

OF THE GATHERING OF THE COCOONS, AND THEIR MANAGEMENT FOR SALE OR REPRODUCTION

There is scarcely any thing, amongst the various wonders which the animal creation affords, more admirable than the variety of changes which the silk worm undergoes ; but the curious texture of that silken covering with which it surrounds itself before it arrives at the perfection of its animal life, vastly surpasses what is made by other animals of this class. All the caterpillar kind do indeed undergo changes like those of the silk worm, and the beauty of many of them in their butterfly state greatly exceeds theirs; but the covering which they put on, before this change into a fly, is poor and mean, when compared to that golden tissue in which the silk worm wraps itself. They indeed come forth in a variety of colours, their wings glittering with scarlet and gold, yet they are but the beings of a summer's day, both their life and beauty quickly vanish, and they leave no remembrance after them; but the silk worm leaves behind it such beautiful, such beneficial 
monuments, as at once record both the wisdom of the Creator, and his bounty to man.

The matter out of which the silk is formed is, while in the silk worm, only a fine yellow transparent gum, contained in two vessels as thick as a large knitting needle; these vessels lie close to one another, and communicate with two small orifices below its mouth; on which account it is, that though the silk thread as it is, spun seems only single, yet it is in reality two threads sticking slightly together by their sides from beginning to end, and they may be easily seen and drawn asunder by the help of a microscope, or even without one. This gum is of a particular species, neither dissolvable in water nor spirit of wine, though they will a little soften it, and it receives its firmness and tenacity immediately upon the silk worm's drawing it out in a thread, from the air which dries its moisture.

We take this gum to be of the nature of horn melted into the state of a jelly; for the silk vessel, being taken out of the worm and hung up, will, in one day, become quite dry and hard, not differing in appearance from a piece of tough yellow horn, and having the same smell when burned: thus silk is only an excessively fine hair, with some small portion of gum on its surface, such as water can dissolve, and which 
causes several of these hairs to adhere together when reeled out of warm water, and it is this part of the gum which, by its dissolution, occasions the waste suffered by the silk in boiling; but there is much greater waste than this would occasion when the silk is artificially gummed, as is sometimes fraudulently done, to increase its weight, or to make it lie smooth, in order to conceal what has been ill reeled.

The silk worm can fix and form its cocoon in any angle, or hollow place that is nearly of the same size with the cocoon; it generally roams about for some time amongst the branches, till, having got a fit place, it begins to work by first spinning thin and irregular threads which are to support its future structure; upon these he forms on the first day, a sort of oval of a loose texture, which is called the floss or tow silk; within this, on the subsequent three days, it will complete the cocoon, remaining always on the inside of the sphere which it is forming; during its work it rests on its hind part, and with its mouth and fore legs fastens and directs the thread. This thread is not directed in continued rounds on the inside of the ball, but is spun in spots forward and backward, in a sort of wavy figure; and this is the cause why a cocoon, in winding off its silk, will perhaps not turn once round while ten or twelve yards of 
silk are drawn out. This movement has been admirably imitated in the Piedmont reel, as we shall show in its place.

At the end of four days at farthest, reckon. ing from the moment when they first begin casting the floss, the worms have finished their cocoons, whose inside is generally besmeared with a sort of gum, of the same nature with that out of which the silk is formed, and which seems designed, where they work in open air, to protect them from the rain, for the coccon resists the wet so well, assisted by the silk which is around it, that those balls, when first put into hot water to be reeled off, swim on the top like small bladders, not admitting the water inside unless they are imperfectly formed, or the silk almost reeled off. When the cocoon is finished, the silk worm being now much shortened and wrinkled, so that the rings of its body appear very deep, it rests a while, and then throws off its skin; this may be called the fifth moulting, though not mentioned amongst its others, because it does not interfere with your management: and now, upon opening a cocoon, you would see the worm in the form of a beetle or chrysalis, in shape somewhat like a kidney bean, but pointed at one end, having a brown smooth skin, with rings, and its skin which it throws off lies in the cocoon with it. 
In this form it continues according to the different heat of the climate. It then throws off the chrysalis skin, which may be called the sixth moulting, and has now the complete form of a white butterfly, with four wings, two black eyes, and two horns or antlers branching sideways, like two very small black feathers. It then pours from its mouth a tasteless liquid, nearly like that which moistens and softens the envelope which wraps it, and also the strong web of the cocoon in which it is enclosed. You will know the formation of the moth, and its disposition to come out, by seeing one of the extremities of the cocoon being wet; some hours after these signs have appeared, and sometimes even in one hour after, the moth will pierce through the cocoon, and come out; therefore, lest you should lose your cocoons in this way, it is necessary that you gather them and that the chrysalis should be killed in those which you have not leisure to reel off before the time of the moth's piercing them, after having first made choice of a sufficient number to breed from.

The time in which the silk worms finish their cocoons varies according to the weather; experience teaches that strong, healthy and well managed silk worms, will complete their work in three and a half or four days at farthest, 
reckoning from the moment when they first begin casting floss, as we have already said. You may know whether they have finished by shaking gently the cocoons in your hands, for if the chrysalis is loose and rattles in it, it is a sign that they have done spinning, that is to say, all that began to spin at or near the same time with the one on which you make the trial; but in order to be perfectly sure, you may not gather them until the seventh or eighth days are elapsed; then begin with those shelves where they spun first, taking the branches and twigs down regularly, and pulling the cocoons from amongst them successively.

In gathering the cocoons, you should make four different assortments of them, for which purpose you must have four baskets : in one of these, place gently those which are designed for breed; in another put all those that are double, having two worms in them; in the third put the firmest and hardest of those which are to be reeled; and in the fourth those which are of a looser texture. Those which are very thin, unfinished, or spotted, may make a fifth sort.

Let your choice of those which are for breed, be always from those shelves or arbours where they begin to spin earliest. Choose from these the hardest, particularly when the two extremities are hard, and the web fine; those that are 
a little depressed in the middle, as if tightened by a ring or circle, and not the largest, that vour breed may be strong and healthy. For if you make use of soft and imperfect cocoons, which are the produce, generally, of weak worms, your future broods will degenerate, both in size and vigor, and give you vastly more trouble, and less profit than strong and healthy ones.

In order that the farmer may judge of the quantity of cocoons that it will be proper or advisable for him to put aside and preserve for eggs, it is right that he should be told that sixteen ounces of cocoons will produce about one ounce of eggs, and one ounce of eggs, well managed, will produce from 112 to 127 pounds of cocoons of the very best quality.-Dandolo.

Whether the laburatory be large or small, the produce will always be exactly in the above proportion, and will not diminish, let the season be ever so unfavorable, if the rules we have prescribed, are strictly adhered to.

To return to the cocoons destined for breed, it may be said with full confidence, that if they are taken from a well managed laboratory, it is needless to give one's self much trouble in choosing and sorting them. Several experiments have proved the above fact.

If this choice is to be made, it is recommend- 
ed to select the white ones in preference, and keep the colored ones either for sale or to reel, because the white silk commands always a higher price than the yellow; if you have no white cocoons, then choose the straw-colored ones. Attention should be paid to having an equal uumber of males and females, though there are no certain signs to distinguish the cocoons which are to produce the male moth from those that contain the female; the following are the least erroneous and best known.

The male cocoons, that is to say those which generally contain the male insects, are smaller, sharper at one or both ends, and depressed in the middle, as it were with a ring; the round full cocoons, without ring or depression in the middle, usually contain the female.

After the cocoons have been taken down from the bush, those which are intended for eggs should be stripped of any down or floss that may still hang about them, so that the moth may find no difficulty in coming out. Then, as far as you can distinguish them, put the males on one table, and the females on another, that they may not copulate too soon, and before they have discharged a viscid humor of a yellow reddish color, which prevents their fecundity; they generally discharge this humor in one hour after coming out of the cocoon. Of this opera- 
tion, we will speak more at length in the directions about breeding, which will form the subject of the next chapter.

The rest of your cocoons which are to be reeled, must either be reeled off directly, or, must be proceeded with so as to prevent the moth's piercing them, as you will see presently ; for though the silk of those cocoons which are reeled off immediately is said to appear somewhat more bright and glossy, yet there will be no difference in the goodness of their silk from those in which the chrysalis is killed in order to preserve them, provided you kill them in a proper manner; besides, where you have a great quantity of cocoons, it cannot be expected that you should be able to reel off many before the time of the moth's piercing; therefore you must be careful in preventing this, which, if it should happen, would be an irreparable loss; and thus, by having all the cocoons in a condition to wind them off at your leisure, you will be able to go the more regularly about it, and to reel off their silk to greater advantage and perfection.

Most persons in Europe, where silk worms are bred, do not reel off their cocoons, but sell them to those who make this their business; and there is no doubt but that all manufactures are the more expeditiously carried on, the more 
branches they are divided into; yet where persons who raise silk worms have leisure and conveniency, they will considerably increase the profit if they know how to reel their silk themselves.

The sorting and separating of the strong, hard, and thick cocoons, from the thin and soft ones, as above directed, will be of some service when you intend to kill the chrysalis within them, in order to prevent their piercing, for as this is done by the means of heat, the thick and hard cocoons will require to be placed where the degree of heat is greatest, that it may penetrate more effectually through their thick and hard substance. There are three methods of suffocating the chrysalis in the cocoon : first, by the ardent heat of the mid-day sun; second, by the heat of ovens, such as they have after the bread has been drawn; and lastly, by the steam proceeding from boiling water; in the first and third of these methods, there is no danger of the heat injuring the silk, fand therefore they are to be preferred when convenience permits.

In order to kill the chrysalis by the heat of the sun, lay the cocoons on a linen or cotton sheet, but not too close, or one upon another, and expose them thus to the heat of the sun in open air, when it is perfectly dry, from 11, A. 
M. to 4, P. M., wrap them up carefully in the linen in order that the heat may the better produce the intended effect, and put them for the night in a very warm place; renew the same operation during four days, in that time there is no doubt that the moths will be killed, particularly in warm climates.

The heat of an oven, such as it is after the bread has been drawn, will generally kill them; but this requires nicety and attendance, for if it is too hot, or if they remain there too long, it will scorch and injure the silk, and if too cool the chrysalis will recover; the cocoons should be put in long flat baskets, lined with coarse paper, in order that they may not be burned by touching the sides of the oven; the oven's mouth should be well stopped, and in about an hour after they have been there, it will be time to take them out, and sooner, if upon listening you hear a small crackling noise come from them; after taking them out cover them up in blankets made hot, and when they are entirely cooled, expose them to the air or to the sun, because the heat of the oven will make some moisture ooze out of the chrysalides which are killed, which should thus be dried up, lest it rot or injure the silk; it is necessary to pick out the cocoons spotted by the bursting of the chrysalis, which would communicate 
their infection to all those that may be near them.

The third method of killing the chrysalis, which is by the steam of boiling water, is preferable to either of the others, as it performs the work without any injury to the silk, and also with certainty and expedition; it may be done in the following manner.

Have a broad but shallow wicker basket not made too tight, and of such a size as will fit and go into a kettle or boiler; it should be of such a depth as you find by experience that the steam can penetrate through the heap of cocoons; it should also have a wicker cover, to shut over the cocoons which are put into it. Having filled it with cocoons, shut the cover and let it down to within an inch of the hot water, then cover it with a blanket in order to concentrate more of the vapour, and by thus increasing the heat bring the operation sooner and more effectually to a close. After it has remained in this situation five or six minutes, which is sufficient to kill the chrysalides at the bottom of the basket, take it out, and turning it upside down, fix it in the same manner as before, over the steam; five or six minutes more will be sufficient to kill the chrysalides of those cocoons which were before uppermost, but now next to the water. Thus, when they are suffi- 
ciently steamed, pour them out in a heap on a blanket, made very hot, and wrap them up closely (but not so as to crush or flatten them, which is of the greatest importance, ) that by this continued heat you may be more certain of having killed the chrysalides; then fill the basket again and proceed in the same manner until all your cocoons are steamed.

When the cocoons are cold in the blankets in which they are wrapped, they should be spread in the sun, or in an airy place to dry any moisture which they may have gotten from the steam of the boiling water. If the weather is not favourable for drying them out of doors, you may do it by spreading them on the wicker hurdles, where the worms were fed, at the same time opening the windows, that the air may have a free passage; when thoroughly dried, they may be conveyed in baskets to send them to market, or deposited in a place protected from dust, and filth of flies, or rats and mice, which will gnaw and destroy a great many for the sake of the chrysalis.

Having thus brought your eocoons into such a state that you need not be anxious about any mischief happening to them, it is time to give some attention to those which were separated for breed, the method of managing which we shall now proceed to explain. 


\section{CHAPTER VIII.}

OF THE CHANGES OF THE SILK WORM, THE COUPLING OF THE MO'TH AND THE GATHERING AND PRESERVATION OF THE EGGS.

Enclosed in the new habitation which nature has taught him to provide for himself, the silk worm undergoes various changes. His first metamorphosis is into a chafer or beetle, a kind of scarabæus, to which naturalists have given the Greek name of chrysalis, and in the plural number chrysalides. His next change is into a little white butterfly which we call the moth, and is sometimes called by silk culturists the miller, from its color. In this last shape, after a longer or a shorter time, he breaks through his silken prison, again breathes the external air, couples with his female and dies; the female also dies soon after laying her eggs, which may be preserved for a long time without perishing, and at last being hatched by natural or artificial heat, the little animal comes out, not in the brilliant form of its parents, but in their original shape of a silk worm, destined to go through the same labor and the same changes. This is one of the most wonderful works of the Almighty; the 
same creature is successively a worm, a beetle, and a butterfly, in appearance different species and yet the same animated being. We shall not follow here the learned philosophers, who have attempted to explain these operations of the divine will, by chemical affinities and other scientific notions. This work is merely practical, and we leave it to others to penetrate into secrets which we believe inaccessible to the limited powers of the human mind.

Having thus briefly noticed the various changes of the silk worm, we shall now follow the dear little butterflies through the short period of their lives, and show the manner in which they ought to be managed, and their valuable eggs gathered and preserved for reproduction.

There is nothing of greater importance in the management of silk worms than that of obtaining a strong healthy breed, whether you regard the trouble which it will save you in feeding, or the advantage of their silk. Every method therefore, which improves the breed is carefully to be attended to; for when you have once got a good kind, you may easily keep it such, but if you let your worms degenerate and breed promiscuously, the weak with the strong, in a short time you will have none fit to raise a good breed from. 
We have already given directions how to make choice of such cocoons as are proper to breed from, the next thing you must expect to see is the coming forth of the moth. The time of its coming out is various, according to the warmth of the season and climate. If the cocoons selected to produce eggs are kept in a temperature of $66^{\circ}$, the moths begin to be hatched after fifteen days; if they are kept in a heat between 71 and $73^{\circ}$ degrees they begin to come forth after eleven or twelve days.

This law is for the most part general, though there are some exceptions ; it is however a sign that the moths are about to come forth when the cocoons are humid or wet at one end, which is that where the head of the moth is situated.

The room in which the moths are produced should be dark, or at least there ought to be only sufficient light to enable one to distinguish objects. This is an important rule, and must be carefully attended to.

The moths do not come forth in a great number the 1st nor the 2d day; they are hatched chiefly on the $4 \mathrm{th}, 5 \mathrm{th}$, 6 th and 7 th days, according to the degree of heat of the place in which the cocoons are kept.

The moths generally come out in the morning, and cling with their feet to the outside of the cocoons. 
When you choose the cocoons for breed, we advise putting the males and females on separate tables, because if they were on the same, they would begin to flutter about and couple as soon as they came out, and would hinder that regularity which you should observe in putting them together. You may chance indeed to mistake some males for females, and the contrary, while they are in the cocoons; but this is of no consequence, for when the moths come out they are easily distinguished, the female having its body nearly double the size of that of the male, its color somewhat whiter, and horns not so black, nor so large as those of the male; besides the difference of shape, which in the body of the male is more slender and sharp at the end, you will observe him fluttering his wings with great quickness, and moving about with activity; whereas the female remains very quiet, and moves her wings very little.

When the moths have had leisure to get rid of the humid and earthy matters that load them, take them gently from off the cocoons and place them by pairs, a male and female together, on a piece of smooth woollen stuff, no matter how old or ordinary, about a couple of yards broad every way, or in proportion to your number of moths, and take care to darken the room. 
You may, in thus pairing them, put the strongest together, and you may quite reject those that are weak, or some way injured, by which means you will further meliorate the breed.

Separation of the moths, and laying of the Eggs.

In what we have said above, we have supposed, when speaking of the coupling of the moths, that the number of males was equal to that of the females; if that were the case, it would only be necessary at the time of their separation, to keep the females, and throw away the males; that, however, is never the case, as there is alwaysan excess either of males or females; in the first instance, you must preserve the females in a spare box kept in darkness, and males which you have separated from their females, must be put to them; in the second instance, if there are more males than females, the surplus must be thrown away.

When the moths have remained thus coupled about six hours at most, you take them by the wings and the body, and separate them gently, which is easily done; the males may be thrown away as of no further use, unless you happen to have a superfluity of females, in which case you may keep a few of the males, which remain most vigorous to pair with them, having had the precaution to have allowed 
them to remain with the females, the first time, five hours only instead of six.

To preserve the males in a state of vigour till the moment of coupling comes, they must not be allowed to flutter with their wings, but be put in a small box. Before separating the two sexes you must prepare, in a cool, dry, and airy chamber, the cloth on which the female is to deposit her eggss. Twenty-two square inches of cloth are sufficient to contain on its surface six or seven ounces of eggss.

The reason for separating the males and females, and not waiting till they uncouple of themselves, is because the vigor and the life of the females being now of short duration, they should have time to lay their eggss while they remainin strength, whereas, if you have left them to uncouple of themselves, they would freqnently not do it in less than two days, especially in the more temperate climates.

You should visit the moths at tirnes, while they are paired, and should bring together those that wander out of the way, and those which uncouple too soon, that is to say, in an hour or two, that they may pair a second time.

Before the moths are unpaired, whether they do it of themselves, or by your means, you should have in readiness proper materials for the females to lay their eggs on. The fittest 
things for this purpose as has been said before, are pieces of the smoothest sort of woollen stuff, this is better than paper or linen; for to these the eggs will stick so fast, that it will give you vast trouble to get them off, which cannot be done without bruising many of them.

When every thing has been thus arranged, recollecting that the room should be dry, and all light excluded from it, except what is necessary to see what you are about; the moths that have been paired six hours are to be gently separated, the females to be placed on the pieces of the described stuff, one after the other, beginning at the top, and going downwards. This operation must be continued without intermission, as long as you find females that have been paired during the necessary time. The time must be noted at which the moths are placed on the cloth to couple, taking care to keep those which are placed afterwards separate, to avoid confusion.

As we have already said, the time when the greatest number of moths comes out, begins about six or seven o'clock in the morning. Consequently the coupling takes place about nine o'clock, and at three in the afternoon the males ought to be detached, and the females deposited in the place above described, and left on the pieces of cloth thirty-six or forty hours, without being touched. 
By attending to the above directions, the three following qualities of eggs may be obtained on separate pieces of cloth.

1st. The eggs of the females that have coupled with virgin males.

2d. The eggs of females that have coupled with males who had already coupled with other females.

3d. Those of females, which after thirty-six or forty hours, continue to drop their eggs.

On these smoothest pieces of woollen cloth, the moths will lay their eggs, but there is a great difference in the time employed in so doing; and according to their strength and fecundity, the number of eggs is more or less. The eggs stick, to whatever the moth lays them on, by a natural gum with which they are smeared; they are first of a pale yellow color, then greenish, afterwards they grow somewhat red, and in about twelve or fifteen days after being laid, they attain a blueishgrey color sooner or later, as the weather is favourable; and of this color they always remain, unless they afterwards happen to be damaged by too much cold, heat, or moisture in keeping them. Those which do not get this blueish-grey color, but remain reddish or yellow are either unimpregnated or imperfectly 
so, therefore are good for nothing, as generally they do not produce worms.

When the season, or the temperature of the room is too hot, that is to say, when it rises to $78^{\circ}$ or $80^{\circ}$, or when it is too cold, as for instance, $64^{\circ}$ or $66^{\circ}$, you will find more or less of yellow unimpregnated eg'gs, or of a reddish color imperfectly impregnated, the temperature of the room must therefore be kept between these extremes.

Whether the eggs be or be not impregnated, or only imperfectly so, they are always of a lenticular form. A little time after their production, there takes place, in the centre of both surfices, a depression, which proves that a portion of the aqueous part of the egg has been disengaged, and that a lind of drying process has been effected.

In twelve or fifteen days, according to the different degrees of temperature of the rooms, the eggs undergo almost all the gradations of color mentioned abore, and then possess the character of impregnated eggs. All these changes of color come from the fluid of the esgs, and not from the shell, which is almost transparent.-(Dandolo, chap. v.)

When all these operations are finished, there is nothing else to attend to, except the pre- 
servation of the eggs. We conclude this subject by observing that at this time, the impregnated female, which weighed about thirty grains, in three or four days after having deposited its eggs, weighs only about twelve grains. When dead and dried up, its weight is only three grains and a half.

\section{Preservation of the Eggs.}

When the eggs have acquired the color proper to them in the perfect state, and the cloths are quite dry, it is time to think of the means of preserving them. From the pieces of cloth you can with great ease separate the eggs, in order to preserve them until they are to be hatched in the ensuing spring, or the cloths may be folded and placed on a net work and attached to the vault or ceiling of a cool and dry place. In this manner the cloths have air on all sides, the mice cannot get at the eggs, and they are well preserved. They must be inspected nearly every month in the winter, and every ten or fifteen days in warm weather.

In all cases the eggs are to be placed in cool, and perfectly dry places, the temperature of which, should not much exceed $65^{\circ}$, nor descend below $32^{\circ}$; for moisture rots many of the eggs, and sometimes destroys entire broods; on this account, therefore, those who let them 
be laid on paper or linen, and keep them so all the winter, will have many decayed ones, for paper and linen are both apt to attract moisture. Heat is to be avoided, because it might bring the eggs to hatch before their time; and great cold would be as hurtful to them as moisture. A bedchamber therefore, is a proper place to keep them in, but not near the fire; and if the weather grows warm before the mulberry leaves open in the spring, they should be removed into a cooler place, to retard them until there is food for the worms. Observe also, that if the weather is cold or moist when the moths are put to couple or to lay their eggs, it should be done in a place with a fire in it, otherwise they will not lay so many nor so good.

If you are afraid that it should freeze where the eggs are stored, a thermometer must be put there, or a little water in a bowl. If the water does not freeze, the cloths may be safely left there. If it is feared that the place is not dry, it may be ascertained by using a barometer, or by putting common salt in a dish. 


\section{CHAPTER IX.}

\section{REMARKS ON DIFFEREN'T VARIE'TIES OF SILK WORMS.}

As there exist several varieties of silk worms, and as the term of life of these various species is not the same, and their silk is of different value, it is necessary to explain those differences, and to examine the advantage or perhaps the loss that might arise from rearing any particular sort of silk worms, the result of which may be of some importance.

To state this clearly, we shall speak,

$1 \mathrm{st}$, Of the small silk worm of three casts, or moultings.

$2 \mathrm{~d}$, Of the large silk worm of four casts.

$3 \mathrm{~d}$, Of the common white silk worm of four casts.

4th, Of the common yellowish silk worm of four casts.

1st, Of the small sille worm of three casts.

The eggs of this species weigh one-eleventh less than those of the common silk worm, for 39,168 of the latter make an ounce, while 42,620 of the former are required to make that weight; 
the silk worms and cocoons of this cast are twofifths smaller than those of the common sort. Experience has shown that these worms consume, for each pound of cocoons, nearly as great a quantity of leaves as that eaten by the common sort, and, although smaller when they have reached their highest growth, they devour more fragments of leaves, than the latter, so that in cultivating the common sort, we lose fewer leaves.

The cocoons of the small silk worm furnish finer and more beautiful silk than the common cocoon, as 506 feet of the single thread or fibre of silk from worms of this species, weigh one grain, while 458 feet only of silk from a cocoon of a worm of four casts, make the same weight. It would appear, therefore, that in these silk worms the silk-drawing tubes are finer. The cocoons of this variety are better formed, and to this is owing the greater quantity of silk which, at equal weight, is drawn from these cocoons, in proportion to that afforded by the common cocoons.

It is evident therefore, that what has been said, tends to show that this variety of the silk worm ought to be cultivated, because those who buy silk, knowing its superior quality, will give a higher price for it. Thus will trade be benefited, and the industry of the cultivator 
encouraged. Besides these advantages there are some others equally important.

1 st. These silk worms require four days less of care than the common silk worm.

2 d. Therefore, the mulberry trees may be stripped sooner; they shoot faster and resist the cold weather better.

3d. They afford a saving in time, labor and money.

4th. They are not so long exposed to accidents or contingencies, their life being shorter.

Some imagine that as 600 cocoons of this species weigh a pound and a half, and 360 of the common cocoons make the same weight, they eat more than the latter, but experience shows this to be erroneous.

The eggs of this species may be found in several parts of France, particularly in the south.

2. Of the large silk worm of four casts.

I reared, says Dandolo, many of these silk worms of a very large quality ; these eggs produce larger worms and larger cocoons than the common species; they are only one-fiftieth more in weight ; 37,440 eggs weigh an ounce, whilst 39,168 eggs of the common sort make the same weight.

The worms proceeding from those eggs, 
weigh, when they have attained their utmost size, nearly twice and a half as much as the common worm. The cocoons are in the same proportion; 150 of the largest sort weigh a pound and a half, while it requires 360 of the common cocoon to weigh as much.

The only advantage they may offer, is that $18 \frac{3}{4} \mathrm{lb}$. of leaves will produce $1 \frac{\mathrm{I}}{2} \mathrm{lb}$. of cocoons, whilst $20 \frac{3}{4} \mathrm{lb}$. of leaves are required, to produce $1 \frac{1}{2} \mathrm{lb}$. of the common cocoon. But this advantage is greatly diminished because:

1. The silk of these cocoons is coarser and not so pure; this explains the reason why the worms consume fewer leaves; 421 feet eight inches of the silk from the great cocoon, of large worms of four casts, weigh a grain, whilst we have seen that 506 feet of silk from worms of three casts weigh as much, and that it requires 458 feet of silk from a common cocoon of four casts, to give the same weight.

2. These worms are five or six days later, in attaining their utmost growth, and in rising, than the common silk worm.

3. The cultivator runs the chance of stripping the mulberry trees later, and injuring them.

4. The labourers must be kept on longer, which incurs expense.

5. The insects are exposed to more danger and risk, as their life is longer. 
Consequently, this variety and species does not suit climates and regions similar to those of the Eastern States; but they may possibly answer better in warmer States, and in different circumstances.

3. Of the common white Silk Worm of four casts.

'This species of silk worms may be reared with advantage; they are in all respects equal to the common silk worms of four casts, but their silk is more valuable than the yellow silk. The whitest cocoons should be carefully selected for the production of eggs.

The silk worms that spin white silk deserve the observation and attention of the cultivator. We would particularly recommend to those who rear silk worms for the purpose of reeling the silk themselves, to cultivate only the small silk worms of three casts, and the silk worms that spin white silk, as preferable to all others, and consequently to choose every year the very whitest and finest cocoons for eggs, to prevent the degeneration of the species.

Of the common yellowish Silk Worm of four casts or moultings.

This species, though not the best, is the most generally cultivated. Of this kind, however, 
the best are those that form a pale straw-colored cocoon, which is preferable to the deep yellow. Although it requires $20 \frac{3}{4} \mathrm{lbs}$. of leaves of the mulberry tree to obtain a pound and a half of these cocoons, the cultivator prefers them, for the convenience of always having his own eggs, more from habit, than any thing else. 


\section{HAPTER X.}

OF 'THE DISE $\triangle$ SES OF SHLK WORMS.

Without reckoning those accidents, already mentioned, by which the eggs become decayed before they are put to hatch, the silk worm during the time of its formation in the egg, that is, during the time of hatching, is subject to accidents and mortality: these have been partly guarded against in the directions given for making the eggs hatch.

The silk worm being a robust animal by nature, although its life lasts but a few days, it would appear impossible that there should have been written hundreds of works upon the subject of its diseases. If we would cxplain why so much has been said upon this matter, we should find strong evidence that it is because the disorders have been looked upon as constitutional, and that it has not been considered that most of them arose from the ill management in rearing they have undergone.

This, however, is the real case, want of attention and sufficient care is the cause of most of those diseases on which so much has been said and written. If we only manage them 
properly, and do not neglect them, we may be almost certain of never seeing them attacked by disease during the short time they require before they attain the period when they pour out their silk. This precious produce, may become one of the most abundant sources of wealth to the United States.

The directions we have given in the preceding pages should suffice to preserve the insects from almost all the disorders incident to them. This chapter, is particularly devoted to demonstrate the truth and usefulness of the system we recommend. We must here repeat, that whenever our rules are strictly observed, the worms will hardly ever, we would almost venture to say never, experience disease; and that on the contrary, whenever those are departed from, they will be exposed to the attacks of those disorders we are going to describe.

Too much precaution cannot be taken in order to prevent the ill consequence which may arise from an unskilful method of hatching eggs ; for moisture may hurt them as much as an improper degree of heat or cold.

Some few worms are liable also to die in hatching by not readily getting out of the shell, which, as it is not fixed to any thing, is apt to be dragged after them in their efforts to get out, till growing tired they are not able to ex- 
tricate themselves; this however, happens to but a few, and those, perhaps, weak worms. But the method in which the eggs must be hatched makes this unavoidable, neither is it of great consequence.

It is likely that eggs often are hurt by not being washed, for then many of them will remain smeared over with that moist substance which the moths cast out a little before they lay their eggs, and this would stop their pores as has been said. It might be well to wash them as soon as they have got their grey color, before they are laid up.

The diseases incident to silk worms after they are hatched, proceed generally, either from their food or the temperature of the air in which they live. An error may be made either in the quantity or the quality of their food. We have formerly mentioned the manner of regulating the quantity to be used; to avoid giving them too much, it will be a good and sure rule if after they have eaten all their leaves, they are left without any food for ten or twenty minutes. But if by any accident they have fasted too long, it is safest to give them sparingly and often for sometime after, and then of the wholesomest and best leaves. And if they are surfeited by over-eating, let them fast 
a longer time, and afterwards feed them also sparingly.

Silk worms are much more liable to suffer from the bad quality of leaves, than the quantity. The leaves of trees which grow in moist grounds, or in places shaded from the sun, are unwholesome; and those which grow on suckers produced from the trunk, or root, being full of sap and moisture, crude and immature, will produce a most dangerous and fatal disease in silk worms: even by only giving them one feeding, they grow immediately surfeited, and throw out of their mouths a greenish liquor, and a clear humour out of the pores of their skin, particularly out of the little point which grows near their tail. This viscous moisture by their rubbing against one another closes up the vents by which they breathe, which are those black specks that appear down cach side; and they seldom recover from this disorder, so as to make cocoons worth any thing. It is therefore much better to avoid this disease than to attempt curing it ; however, as all may not be alike infected, pick out those which are in the worst conditions, and if you have a mind to make experiments on them you may. Then make the hurdles of those that remain, clean and dry, and letting them fast two or three hours, give them afterwards some of the 
best leaves you can select, but few at a time, and gathered a good while before. The mois- tened leaves, would also be hurtful.

Mulberry trees may also have peculiar diseases in themselves, which may make their leaves unwholesome for silk worms, but this will be best discerned in the growth of the tree and of its leaves, and the diseases incident to the silk worms, more easily avoided by not using them. Air is the next thing to be considered, and a number of cases have shown how air affects animals, and how, by a variety of incomparable contrivances, it may best be turned and tempered to the use of life.

Silk worms are more liable to be affected by the air, which is perpetually necessary to animals, than by any other circumstance of their lives; sudden changes from heat to cold, and from cold to heat, are very prejudicial to them, and such climates as are much subject to such variations, dangerous to them. These changes, however, can be tempered by art and contrivances, because silk worms are kept within doors; but putrified air occasioned by the worms being either kept too close, or not having their hurdles cleaned, is apt to destroy great numbers; yet the cause of such distemper is easily seen; for the abundance of their litter, and the smell which it spreads over the room 
are sufficient indications that the hurdles want cleaning, and that the room wants a supply of more wholesome air. Indeed, very little contrivance would keep the room constantly supplied with fresh air, which being a thing of the utmost importance, we shall here mention an easy method of effecting it, founded upon that remarkable property of the air, that when purest, it is always most heavy; and when mixed with vapour, either putrid, aromatic, or watery, or indeed with almost any thing that affects the sense of smelling, it is lighest.

Now, in any room where silk worms are fed, if an aperture, about nine inches square, be made in that angle of the ceiling which is farthest from the door, then will the putrid vapours, occasioned by the litter and the multitude of worms, constantly ascend through this aperture, and the pure air will succeed by the door in its place; or rather, if an aperture is made towards the bottom of the door, of the same size with that in the ceiling with a small shutter to open and close as occasion may require; then will the fresh air come into the room with greater advantage, by raising up, and carrying off the foul air above it.

It would be better if several of these apertures were made in the opposite walls of a room, near to the floor and in the ceiling open- 
ing into the external air, they would have this convenience, that you can take the advantage of opening the apertures, which are on the windward side, when the room wants to be suddenly and thoroughly cleared of the foul air.

Thus, if the proper cleaning of the worms, and the due admission of fresh air be practised, the diseases arising from dirt and putrefaction will be prevented, so far as they arise from the manner in which people are obliged to keep silk worms in houses; and, unless such cleanliness and airing of the rooms be observed, there will be always complaints, (as there are at present, in the countries which breed silk worms,) of an unaccountable mortality amongst the worms of some persons, while those of others remain healthy. But the smell of the room will give warning of the danger, and point out the best cure, which is prevention.

It is true, indeed, that diseases of the same kind may arise from some peculiar putrified state, or malignancy in the atmosphere, as in hot and moist summers, or in places abounding with mineral exhalations. But then the disease will appear more universal, and will more or less affect all the silk worms in adjacent places; in this case, often clearing the litter away will contribute to preserve the worms, as also not keep- 
ing them too much thronged and crowded together. The malignancy of the air may be purified in the following manner.

Of all the methods employed for purifying the internal air of the room, and of neutralizing and destroying, in some degree its deleterious qualities, the following is the surest and the cheapest.

Take six ounces of common salt, mix it well with three ounces of powder of black oxide of manganese; put this mixture in a strong bottle, with two ounces of water, cork it well with a common cork. Keep this bottle in any part of the room farthest from the stove or fire place. In a vial, put a pound and a half of sulphuric acid, (oil of vitriol) and keep this vial near the other bottle, with a small cordial glass, and an iron spoon; this is the manner of using it: Put into the small liquor glass, two thirds of a spoonful of oil of vitrol, pour it into the large bottle, and there will issue a white vapour. The bottle should be moved about through the room, holding it high up, that the vapour may be well spread in the air.

When the vapour ceases the bottle may be corked, and replaced; even should there be no perceptible difference between the interior and exterior air, during the fifth age of the worms it is good to repeat this fumigation, three or 
four times a day, in the manner just explained. When repeating the fumigation, the quantity of oil of vitriol poured into the large bottle may be diminished. The bottle may be left open an hour or two in the last days of the fifth age of the worms, and placed here and there in the laboratory, and even on the corners of the wicker hurdles, to diffuse the vapour thoroughly.

This easy method may be employed whenever on going into the laboratory the air appears to have unpleasant effluvia, and there is any closeness or difficulty of breathing; it is more powerful than all the perfumes commonly used, and produces five advantages in the laboratory.

1st. The vapour, in spreading immediately, destroys all unpleasant effluvia.

2d. It diminishes the fermentation of the litter, and dries it up.

$3 d$. It neutralizes the effect of all the miasmata, and deleterious emanations that might affect the health of the silk worms.

4 th. It revives the silk worms, gently stimulating them, because it is composed in a great measure, of pure vital air.

5th. This vapour is not alone favourable to the health of silk worms, but has some effect on the goodness of the cocoon.

Care should be taken not to drop any of the 
vitriol either on the skin or the clothes, as it burns, and to hold the bottle above the height of the eyes and nose, when it is open, because the vapour is very searching, and would be dangerous and unpleasant.-(Dandolo, chap. 7.)

If you cannot get manganese, nor procure it well pounded, then put ten ounces of nitrate of potash, (common nitre of the shops, ) into a bottle, instead of the common salt and manganese, used in the former composition, and follow up the other parts of the recipe. The nitre should be quite damp, and a smaller quantity of the vitriol than has been indicated may be poured into the bottle to make the fumigation.

The gas proceeding from this last composition, is very similar to the other; it is less subtile, and not so dangerous. It is composed of vital air and nitrous vapour ; it quickly destroys all animal exhalations which may exist in the atmospheric air.

When silk worms become sick and languish by a continuance of moist weather, it is difficult to relieve them, it being as yet a desideratum amongst inventions, to supply quantities of dry air from a moist atmosphere. Fires may indeed warm the air, ard so hinder the bad effect of its dampness ; but still the air which is constantly drawn into a room where there is a fire must be supplied by the atmosphere, and thus bring 
its moisture along with it. It is best in this case to admit no more external air than is necessary to keep that within fresh. It may also. be of use before you feed the worms, to scatter over them some very dry straw or hay, so thin that they can pass through it, and upon this to strew their leaves, this may imbibe part of the moisture which is in their litter, or on the surface of their bodies, and at least it will keep them from lying upon their litter when there is a good deal on the hurdles, and you have not leisure to cleanse them immediately.

Silk worms sometimes die during their spinning, or after they have finished their cocoons, before they change into chrysalides. The proper regulation of heat, cold and fresh air in this case, is all that is in your power by way of prevention, and this has been mentioned in its proper place. When the worm dies before it has finished its cocoon, it never rattles in it, upon shaking the cocoon; therefore such cocoons must never be chosen for breed.

Such diseases of the moths as are within your reach, are only languor in coupling, or in laying their eggs, occasioned by the coldness or moisture of the weather, which indicate warmth and a dry place as the proper cure.

Thus a great number of diseases will never appear, 
1st. If the silk worms are kept thinly spread on the wickers, that they may breathe and perspire freely.

2d. If the interior air of the room is constantly and evenly maintained at the temperature we have recommended.

$3 \mathrm{~d}$. When the air is never allowed to stagnate in the room, and it is kept in a gentle, slow motion.

4th. By constantly burning blazes when the exterior air is damp and stagnant, and the interior evaporation superabundant.

5 th. When the room is kept light; light being the most powerful excitement to living nature.

6th. By never having the litter liable to fermentation longer on the wickers than has been prescribed.

7th. By being careful never to distribute leaves that have not been thoroughly dried.

8 th. By using the fumigating bottle when needful, the vapor of which destroys the most noxious animal emanations.

These things will be sufficient to prevent the occurrence oî any of those diseases. 


\section{CHAPTER XI.}

OF COCOONERIES.

As it may happen that some persons will be disposed to pursue the silk culture upon a large scale, we will give, hereafter, the description of a building on the model of Count Dandolo. But, as we have already said, we mean principally, to speak of the popular pursuit of it, as the incidental occupation of females of all ages, of children, and of old and poor people; and which, nevertheless, produces great results, as well in regard to the number of worms intended to be reared, as to the raw silk to be produced.

This popular pursuit requires no special buildings or establishment, which never fail to be expensive. We speak of the culture of silk as an incidental occupation, which can be followed in any convenient rooms; and we have raised many thousands of excellent cocoons in an upper room in the city of Philadelphia.

The rooms ought to be dry, and sheltered from the extremes of cold and heat; as the worms dislike dark rooms, they ought to be sufficiently lofty and light. The temperature of 
the climate is therefore to be regarded, so as in hot climates to avoid violent heats, and in temperate ones severe colds, and dampness in all. The windows of the rooms in which the worms are kept should be left open, when the weather is fine, (but never when it is windy or when it rains,) so that the air may freely circulate, in order to refresh and clear the place of bad air or smell. These windows, provided they shut close, may be made either of thin canvass, which is best in warm climates, or of glass, oiled paper, transparent membranes, or any other material which will exclude the cold and admit light. No bad smells, such as those of smoke, sewers, or the like, should approach their habitation. Air holes, of twelve inches square, with moveable slides, will be very serviceable, and they ought to be made near the ceilings, or under the windows, or at the door, near the floor. 'The exterior air penetrates through the lower holes, and carries off through the upper opening, the air which before filled the rooms; the number of these holes must be proportioned to the size of the rooms. Experience shows that this is the best means of renovating the air of an enclosed place; and in order to keep it in general as pure as possible, there ought not to be too many worms in a single room; their perspiration being very great, 
and consequently, the medium in which they live, is soon affected by this cause.

While silk worms are very young, they take up but very little room, and during the first week at least, may be kept in shallow broad drawers, boxes and such like, and that in great number; for a foot square may contain above five thousand of those newly hatched, but as they grow larger, so must also the space that . contains them. Those therefore who have large nurseries of silk worms, should provide a place large enough to hold them when at their full size; a foot square will not contain above one hundred worms conveniently, when they are full grown.

With the foregoing conditions, it is no matter what sort of buildings the silk worms are kept in; the barns and other out houses of farmers may be used for this purpose ; or in hot countries, occasional sheds may be made, which, in such climates as some of the southern states enjoy, will sufficiently answer the end ; for the reader must here take notice, that several circumstances which have been mentioned in the course of these instructions, are rather conveniences for those that would do things in the neatest manner, than absolutely necessary, and that many of the precautions against cold and wet, will be useless in some climates. Those 
circumstances which are absolutely necessary to be observed, the practitioner will, from his own experience, soon distinguish from those which are not so material, or which the nature and conveniences of the country determine him to make choice of ; and the course of his practice will also make him supply what may happen to have been forgotten amongst a number of minute and various details. 


\section{CHAPTER XII.}

\section{EXTRAC'T FROM THE WORK OF COUNT}

DANDOLO, ON THE SUBJECT OF THE PRECEDING CHAPTER.

"It is difficult to imagine how, in the lapse of some centuries, the practice of the useful and precious art of rearing silk worms should have remained in the hands of the ignorant and illiterate.

6" While it is evident that the abundance and certainty of the annual produce of the cocoons rests entirely upon the perfect cultivation of the silk worm during the various periods of its existence, and as it is generally known that these insects are not natives of our climate, and only exist by the care bestowed on them in their domestic state, it can scarcely be credited that there should not yet be a code of sure rules to form habitations suited to their wants, and favorable to their progress, and that silk worms are to be exposed to every circumstance most injurious to their health and well being.

"It seems never to have been imagined that four or five ounces of eggs would produce 150,000 , or even 200,000 silk worms, that would 
all require room to breathe freely the pure air, and to secrete the substances necessary to life.

"A building wisely constructed, upon the fixed principles of art, where the air may circulate at all times, and in all cases, and preserve its dryness, would alone powerfully contribute to the constant prosperity of the animal, and, in course of time, to the abundant production of cocoons of the finest quality.

${ }^{66}$ When the habitation of the silk worm has been well prepared, an unspeakable advantage has been obtained, and all will probably advance favorably.

${ }^{66}$ As we must suppose that many proprietors will have laboratories constructed, so as to insure an abundance of cocoons, I will here give a short detail of the construction of a laboratory, and point out a few indispensable alterations and reforms that may be made in the laboratories of tenants and cultivators, which are already built; and these reforms are infinitely to the advantage of the proprietors and cultivators.

"6In speaking of these two classes of laboratories, I must also mention one of the most necessary appendages to such establishments, the places required for preserving the mulberry leaves fresh and good during two or three days, by which means the loss which might occur from being obliged to feed the worms upon wet, 
withered, or fermented leaves, will be entirely avoided.

"If the construction of the building which is to be occupied by the silk worms during their lives is of importance, it may not be useless to give a description of the utensils and tools most likely to facilitate all necessary operations which are to be executed.

"We shall, therefore, in this chapter, speak:

1st. Of the laboratory of the proprietor.

2d. Of the laboratory of the cultivator, or tenant.

3d. Of the proper place for the preservation of the leaves.

4th. Of the utensils.

1st. Of the laboratory of the proprietor. .

"It is a certain fact, that men scarcely ever. employ capital excepting in the manner most likely to bring in rent, or interest in money, pointed out by habit and local circumstances, with the further exception of the expenses of luxury, \&c.

"For instance, if a proprietor purchase fifteen or twenty acres of land, which cost him three or four thousand francs, (\$600, or 800 ,) it is with the view of drawing one hundred and fifty or two hundred francs from it, (\$30 or 40 .) Admitting this position, it is clearly evident, 
that if we can prove to the proprietor that in employing capital to the amount of three thousand francs in constructing a laboratory of silk worms, he would derive more than five per centum, we cannot doubt that he would endeavor to do so.

${ }^{6}{ }^{\prime} \mathrm{I}$ 'o be able to say with assurance, that it is profitable to construct a laboratory for silk worms, I shall make only one observation, which is, that to draw 150 francs from 3000 francs capital employed in erecting a laboratory, it would be sufficient, that the proprietor received 90 pounds of cocoons more than he annually obtains in the common manner of managing silk worms.

"Are there not, besides, many proprietors, who might arrange, with very trifling expense, such places as garrets, warehouses, $\mathcal{E}$ c., so as to be on a good plan for the rearing of silk worms?

"Can any liberal proprietor, who has informed himself upon the improvements I have suggested in the art of rearing silk worms, state whether he does not think that from ten ounces of good, well-preserved eggs we may easily draw 150 and even 300 pounds of cocoons beyond what may be procured in the bad and ordinary manner of managing silk worms?

6. 'This interest, derived from such an amount 
of capital, is still very trifling, when compared with the other manifold advantages which the proprietor will derive from the erection of a laboratory. To be convinced of this, it is necessary to observe:

61st. That the erection of laboratories tends to increase the value of the lands in which they are erected, and from which the leaves are procured, because they afford a greater produce in cocoons, as land which, when well worked and dressed, gives eight for one, is more valuable than land which will only yield six.

${ }^{66} 2 \mathrm{~d}$. 'That in uniting in the same establishment all the operations relating to silk worms, there is a vast saving in leaves, in fuel, and in labor.

663d. That the common cultivators very soon get into the proper manner of managing a laboratory, when they see the favorable results produced by the prescribed rules, and because they find themselves compelled to act in obedience to the directions of the enlightened persons at the head of the establishment.

"64th That as some of the inequalities in the advantages obtained among the common cultivators disappear sooner than others, they will soon adopt the same means that are invariably secure.

${ }_{66} 5$ th. That the proprietor and cultivator as- 
sured of success, will not neglect the culture of the mulberry tree, nor destroy them, as is often done in those parts where the proprietor strips his trees to sell the leaf, in the persuasion that this is more profitable than employing it himself.

$\because 6$. In short, that the space allotted to the worms is sooner clear and free of them, and may be made use of for other domestic uses.

"What I have said here will demonstrate the profit resulting from proprietors erecting large laboratories.

"My laboratory is constructed on principles which I shall describe, and can contain the produce of twenty ounces of eggs of silk worms, that is to say, might yield $20 \mathrm{cwt}$. of cocoons. It is about 30 feet wide; $\tau \tau$ feet lons; about 12 feet high, and, to the top of the roof, 21 feet. Six rows of tables, or wicker trays, of about two feet six inches in width, may be placed in the breadth of the room, two and two, leaving four passages or avenues between these three rows, two next the two side walls, and two between the wickers. These passages are useful in giving free access to the wickers, and for placing the steps in feeding the worms.

"There should be posts driven in between the wicker trays, which, as I said before, form 
an arenue. On these posts should be fastened little bars of wood, horizontally placed, which support the wicker hurdles: there is, between the two wicker hurdles a space of about five inches and a half, to allow the air to pass ireely, and this space corresponds to the size of the vertical posts, which form a line at equal distances down the laboratory.

"There are in the building thirteen unglazed windows; with Venetian shotters outside, and with paper window-frames inside; under each window near the floor, ventilators, or square apertures of about 13 inches, which may be closed by a neatly-fitted sliding pannel, so as to enable you, at will, to make the air circulate, which, entering and issuing; will blow over the whole floor.

$\because$ When the air of the windows is not wanted, the paper frames may be kept closed, the Tenetian shotters, may be opened or shut, according to circumstances. When the air is still, and the temperature of the interior and exterior are vearly equal, all the window frames may be opened, and the Tenetian shutters must be closed, at least the greatest part of them.

"I made eight rentilators on two lines in the floor, and in the ceiling; placed perpendicu. larly opposite each other, in the ceritre of the pesseger between the wicker hurdles. These 
ventilators had sliding pannels, made of thick glass, to close them and admit light from above, and may also on some occasions be covered with white linen; they may be opened or closed, according to circumstances.

${ }^{6}$ As the air of the floor ventilators ascends, and that of the ceiling ventilators descends, according to the variations of temperature, it must necessarily pass through the three rows of wicker trays.

"I have also had six ventilators made in the floor, besides those under the windows, to communicate with the rooms beneath.

"All these ventilators should open easily when wanted, as they can alone maintain a constant renewal of the exterior air, without the necessity of ever opening the paper window frames, which are within the Venetian shutters.

"I here use thirteen windows, three of which are placed at one end of the laboratory, while at the opposite end there are three doors constructed so as to admit more or less air as may be required. By these doors we are admitted into another hall about thirty-six feet long, and thirty feet wide, which forms a continuation of the large laboratory, and which also contains wicker tray stands sufficiently raised above the floor to facilitate the necessary care of the worms in the laboratory. There are in this 
hall six windows, and six ventilators under the windows, nearly on a level with the floor, as well as four ventilators in the ceiling. There are six fire-places in the great laboratory, one in each angle, and one on each side of the centre.

"I had a large round stove of about three feet eight inches in diameter, and nine feet two inches high, placed in the middle of the laboratory; it divides the large row of the wicker hurdle stands.

"I use small glass oil burners or lamps that yield no smoke, to light the laboratory in the night.

"The floor of the Iaboratory is the only one that is covered with Italian cement (ghiarone,) or stucco; that in the hall is made of bricks, that in case of necessity the leaves may be dried on it when they are wet with rain, and the eggs after they have been washed in the first process.

"Between the hall and the great laboratory, there is a small room having two large doors; the one communicating with the great laboratory, the other with the hall. In the centre of the floor there is a large square opening, which communicates with the lower part of the building. This is closed with a wooden folding door, which may be removed at pleasure; 
this aperture is used for throwing down the litter and rubbish of the laboratory, and is also useful for admitting the leaves of the mulberry which can easily be drawn up with a hand pulley; this same aperture keeps up the circulation of air, when the three window frames at the end of the laboratory are open.

"Such is the construction of my great laboratory, in which I place the silk worms after the fourth casting or moulting.

"It is impossible that the air should remain stagnant in it, or that it should ever be damp; as this building stands alone, the ventilators must by their different exposure keep the air in the utmost equilibrium, and maintain a mild temperature. Should it, notwithstanding all this, have a tendency to stagnation, the air may be instantly put in motion by establishing great currents and burning blazing light fires in the six fire-places; when fires are not needed, nor the chimueys wanted as ventilators, they may be closed with chimney-boards.

"Should there be too much draught, the sliding panels on the ventilators that are under the windows, may be closed, so as to regulate the air as it is required. The same is applicable to the ceiling ventilators, with this advantage, that having a glazed window panel they may be closed and yet give light. 
"The stove is only used when the air of the laboratory wants warming, and the temperature raising; in which ease, when the stove warms the laboratory, a column of external air enters continually into a portion of the stove, which is separated from the part in which the fires burn, and from that through which the smoke issues; in this receptacle, when the air is heated, it escapes, through holes perforated on purpose, into the laboratory, and augments both the quantity of heat and fresh air.

"In different parts of the laboratory I have placed four barometers, six thermometers, and two thermometrograpples, to show what is to be done in case of any accumulation of moisture, and of augmentation or diminution of temperature in the laboratory."

2. Of the Laboratory of cultivators.

In speaking of the establishments of the tenants, Count Dandolo gives the following distressing picture:

"In general the rooms appropriated to rearing silk worms among the tenants, farmers, and common cultivators, have the appearance of catacombs; I say in general, for there are some few who, although they may not have all the requisites for rearing worms in perfection, yet 
have sufficient care to preserve them from any very severe disease.

"I have found, on entering the rooms in which these insects were reared, that they were damp, ill lighted by lamps fed with rancid oil, the air corrupt and stagnant to a degree that impeded respiration; disagreeable eflluvia, disguised with aromatics; the wickers too close together, covered with fermenting litter upon which the silk worms were pining. The air was never renewed except by the breaches which time had worn in the doors and windows."

\section{Of the proper place to keep the leaves of the Mulberry tree fresh and good.}

It is certain that in this conntry writers generally have not suficiently calculated the advantage and damage, which may arise from the leaf of the mulberry, by its proper preservation or neglect, previous to the distribution of it to the worms. Let us hear the Count Tandolo on that important subject.

6" The leaves of the mulberry trees, should be laid on the ground floor, or in cellars, slightly damp, and which may be closed, so as only to admit light suficient to see where to put it, to stir it about, sort, and pick it thoroughly. These circumstances are indispen. sable: 
61 st. Because the lower rooms are always cooler than the upper floors.

$62 \mathrm{~d}$. Because in damp places the leaf is not exposed to evaporation, which alters and withers it. I have kept it three days in such places as I have described : it diminished but little in weight and was not faded. When it is still very succulent, it should be laid in layers of two or three inches, that it may not alter or ferment. When quite ripe it will keep several days, although the layers be above a foot deep, provided it has been gathered when thoroughly dry. It should, however, be carefully moved and stirred about every day, that it may receive the contact of the air, and not get pressed down.

"If in the place allotted for placing the worms, the air ought to be invariably dry, in that destined to keep the leaves, it should be cool, damp, and still. It would be a perceptible loss if the air robbed the leaf of its natural moisture; not so much because it would wither it, as that I consider that natural moisture as a necessary vehicle for the various separations and secretions required for the health of the silk worms, and for the perfect deposition of the silk in the reservoirs. Besides, nature has bestowed much less liquid or watery substance on the mulberry leaf when ripe, than on any other leaf of any tree. 
66 If the allotted space for the preservation of the leaf is very damp, it will not alter it, provided it be cool and well closed; it is heat and accumulation that spoil the leaf. It should be so managed that these places should be under or very near the laboratories; there will be found a great advantage in having a good provision of leaves at hand, particularly at the voracious period, called in France ( $l a$ grande freze ou brife, ) in the fifth age of the worm; and this advantage will be felt still more, should there occur any continued rains at that season."

\section{Of the utensils required in the art of rear- ing Sitk Worms.}

"6 To execute the various operations which form this art, better and with the least possible expense, ought to be the principal object which those who practise that art should aim at; upon this principle I have thought it might not be without usefulness, to give the names of a small collection of utensils which are not expensive, and yet are indispensable in the execution of the operations which the cultivation of silk worms requires.

"This art, has hitherto had no appropriate utensils; each employed whatever came under the hand indifferently. I here give an cxplanation of these utensils. 


\section{Utensils.}

"The Scraper.-A sort of long blunt knife. It is used to detach, or scrape the eggs from the wet cloths by introducing it between the eggs and the cloth, aslant;

"The Thermometer.-It is used to fix the temperature requisite for hatching and rearing silk worms.

"The Stove.-It is intended to warm the laboratory. It heats much better when constructed on the principle of receiving external air, heating it, and then dispersing it in the laboratory. The rarefied air when it comes in hot, is a purifier, as it expels the interior air. The heating the rooms by furnace would be preferable we think, as it would be more easily regulated-besides the apertures through which the rarefied air passes, may be used as ventilators, to admit cold air, when there is no fire in the furnace.

"Small boxes or trays for hatching the eggs of the Silt: Worms.-There should be some of all sizes, that each ounce of eggs may have a space of seven inches and four lines square. If small they should be made of thick pasteboard; and if large, and intended to hold ten or eleven ounces of eggs, of thin board. They should be numbered with very visible figures marked on the sides. 
"Hurdles or trays, or table-stands. - They are used covered with paper-those made with cane, branches, wicker, wood, or basket work, so that it be not close, but so interweaved as to admit of the air being in contact with the paper underneath, which keeps it dry, are preferable; their breadth should be from twenty-nine to thirty-seven inches, their length from eighteen to twenty-four feet, and they should be of equal sizes, that when put above one another, they may not interfere, or extend out, so as to be inconvenient.

"Spoon.-It is made of wood, so as to stir the eggs with care when hatching them.

"Hook.-Small instrument made of bent iron, is very useful to take up neatly and quickly the small twigs covered with silk worms from the boxes, to put them on the hurdles covered with sheets of paper.

Knife-Constructed so as to cut the leaf easily and small; when the leaf is cut with the knife, it should be chopped fine, to multiply the particles and edges of the leaf. This is only requisite in the first and second age.

Large Chopper.-Is made something like straw-cutter; it is useful in chopping the leaf coarsely, and in great quantity. It is used for the three first days after the third moulting.

Barometer.-This instrument is used to measure the humidity of the air in the laboratory." 
We agree with Count Dandolo; we cannot sufficiently urge the use of this instrument to those who rear silk worms, which indicates with so much ease one of the most powerful enemies of the silk worm in the laboratory. It would be desirable to have two of them placed within a certain distance from each other, to ascertain the various degrees of moisture, in different parts of the laboratory. We have omitted, in the above extract, several of the instruments which the Count recommends-we have mentioned only those which we think most necessary.

The above descriptions of a large laboratory, will be valuable to those who may hereafter engage in the business upon an extensive scale. It is easy to perceive that the great objects aimed at are convenience, the preservation of a proper temperature, and the free circulation of air in the laboratory. The American farmer must consider these as cardinal points, whatever may be the dimensions of the building or apartment in which the worms are reared.

We repeat it; all buildings are good for rearing silk worms provided there be, in proportion to their size, one or more fire places, two or more ventilators in the ceiling, and on a level with the floor, and one or several windows or apertures, by which light may be admitted, and sunshine excluded; whichever of 
these methods may be adopted, the rearing of silk worms will be surely practicable; and by the aid of the barometer and thermometer, it will be easy to neutralize or destroy the influence of cold, heat, wind, stagnant air, a damp and corrupt atmosphere, and the fermentation of the litter even may be prevented or arrested. 


\section{CHAPTER XIII .}

\section{MISCELLANEOUS OBSERVATIONS.}

Before we pass to the reeling of silk from the cocoons, which will constitute the third part of this treatise-we think proper to give here some general view and application of the facts stated in this work, which are immediately connected with the art of rearing silk worms.

When an art may become so eminently allied to individual and national prosperity, an author should spare no trouble or labor in order to make the knowledge and practice of every branch of the art clear, plain, and familiar; besides that the facts, when presented together, will make a deeper impression upon the mind, than when they are offered singly. If some of our readers think that many of the things which we are going to state are useless, because they offer no pecuniary results for individual interest, we will answer, that when they reflect upon them they will find that they have more or less connection with those very results which are the 
first aim that the cultirator of silk worms pro. poses to attain-a pecuniary reward.

In Italy, aceording to Count Dandolo, it requires. 37,410 eggs of the largest breed of silk worms of four casts or moultings, to make an ounce, and if all these eggs produced worms. and each made a cocoon, they would yeld three hundred and seventy-three pounds, beeause one hundred and fifty cocoons of this breed weigh ahout one pound and a half: on the contrary; to form an ounce of eggs of common sized worms of four casts or moultings, 3,168 will be required, which all producing worms, and each worm a cocoon, would yield one hundred and sixty-two pounds of cocoons. because about 360 coeoons of this breed weigh a pound and a half: while to form an ounce of eggs of silk worms of three casts or moultings, it will require 42.200 eggs, and if all these eggs produed each one worm, and all the worms lived, the ounee of eggs would yield one hundred and five pounds of eocoons, because 600 cocoons of this breed weigh about a pound and a half. We have already demonstrated the diflerence which exists in the quality of silk drawn from the above different speeies of silk worms; we leave to the culturist to choose between them.

It may be easily aseertained from the above 
facts, and by the quantity of cocoors obtained, how many worms have died in the various ages, or how many eggs have failed to produce worms; and then this will serve to form an opinion on the various methods of rearing the worms, and to adopt the most favorable to their preservation.

There are some other facts not uscless to be recalled in this chapter; they are the following: According to the author above named, it appears that from the time of the moths laying the eggs until they are taken off the cloths, that is to say, in a period of nire months, they lose only alsout $1-100$ of their weight, and that from the days that the eggs of the common silk worms are put into the stove room until they beggin to hatch, they lose, on an average, 47 srains per ource, which is equivalent to 1-12 of their total weight.

The weight of the shells of the egss, after the hatching of the worms, amount to 116 grains per ource, which is about $1-5$ th of the total weight. Therefore, after deducting the loss of weight of the eggs in the stove room, and the weight of the shells, 51,625 silk worms, just hatched, make an ounce, while to make the same weight, 39,168 ergrs were sufficierit.

Following the same author, we will now give some facts relative to the space which should be occupied by the silk worms, in their differ- 
ent ages, and the quantity of leaves consumed by them, with some observations on this last subject. We will show afterwards, the increase and decrease of silk worms in weight and size, and make some remarks relative to the cocoons containing the healthy, the diseased, or dead chrysalis. Next, we will give some facts relative to the production of the eggs, and lastly, some relative to the buildings and utensils.

Facts relative to the extent of space which should be occupied by the silk worms in their different ages, and the quantity of leaves consumed by them, with some observations on this last subject.

As we have already said, the worms proceeding from one ounce of eggs should have space as follows, viz:

Square Feet. In.

In the first age, of second age, third age, fourth age, fifth age,
$9 \frac{1}{2}$

14

109

239

$\mathrm{W}^{\top}$ e would here willingly state the weight of the materials required to form the hedges or cabins sufficient for receiving the worms of one ounce of eggs, that is to say, one hundred and 
twenty pounds of cocoons, but the varieties of brush wood, straw, heath or vegetable substances used for the purpose, differ so much in weight, that the calculation would scarcely be correct, therefore, we will leave this to the sagacity and experience of the cultivator.

It results from a calculation made with the greatest exactness, that the quantity of leaves gathered from the tree, which is really consumed for each ounce of eggs, amounts to 1614 lb., as we shall presently show, viz:

Ibs. oz.

First age, sorted leaves,

6

Second age, do. do.

Third age, do. do.

Fourth age, do. do. 180

Fifth age, do. do. 1098

Per oz. of eggs, sorted leaves, $\overline{1366} \quad \overline{8}$

We must here give some explanation, not having done so in the course of this work, of what we mean by sorted leaves, that is to say well sorted.

Great care must be taken in picking and sorting the leaves for the feeding of the worms in their first ages, such as picking off all the twigs, stalks of the leaves, spots, \&c. and to clear them as much as possible from all useless parts. 
This operation is most essential in the two first ages, when the leaves are to be chopped very small.

In the third age, the sorting and picking the leaves is not of so much consequence, and still less so in the fourth and fifth ages.

The sorting and picking is of importance, inasmuch as it enables you to put 15 or 20 per cent. less substance upon the wickers than would otherwise be done, and which the worms do not eat. This substance increases the litter and the moisture, without necessity or motive.

In the fifth age, and even in the fourth, when the season is favourable, leaves mixed with a quantity of mulberries, boughs and stalks, may be put on the hurdles, although it is known that the worms do not eat them, because at that period it would be too troublesome to sort so large a quantity perfectly, nor is there the same motive to do so. These substances being by this time grown large, hard, and woody, are less liable to fermentation, although they may accumulate as litter. If the laboratories are constantly dry, and well aired, these substances will do no mischief, but keep the litter light, and allow the air to circulate more freely through it.

Let us resume our subject.

The actual quantity of mulberry leaves gath- 
ered from the tree, and consumed for each ounce of eggs, is, as we have already shown,

lb. oz.

But the above quantity of 13668 leaves has lost by sorting so much weight in the following proportion, viz: refuse picked from the leaves,

First age, Second age,

Third age, Fourth age, Fifth age, .

Refuse picked off, per oz. of eggs.

\section{Total of leaves,}

During the whole period of rearing the silk worms, the $1509 \mathrm{lbs}$. of the leaves taken from the tree, will lose by evaporation, and other causes, besides sorting and picking as above stated,

\section{Great total,}

1614

Observations on the above facts.

From the above facts it follows:

1. That it requires about $13 \mathrm{lb} .7 \mathrm{oz}$. of leaves to obtain a pound of cocoons, and that 
it requires $1614 \mathrm{lb}$. to obtain $120 \mathrm{lb}$. which an ounce of eggs should yield.

2. That this quantity of leaves gathered from the tree, deducting $142 \mathrm{lb} .8 \mathrm{oz}$. of refuse and sorting, and 105 pounds of decrease, by means of evaporation, it only requires $11 \mathrm{lb}$. $6 \mathrm{oz}$. of pure leaf, per pound of cocoons, or $1366 \mathrm{lb}$. for 120 of cocoons.

3. That if we deduct from the $1366 \mathrm{lb}$. the residue, such as little branches, stalks, fruit, and fragments of leaves not eaten by the silk worms, and taken off the hurdles with the litter, the quantity of pure leaf necessary to obtain one pound of cocoons will be still smaller ; and consequently that there is a great loss escaped in gas and vapour, in the laboratory invisible to the eye, which present strong evidence of how formidable the enemies are which assail the silk worms.

Although the cultivators in Europe know not the force of the material cause that produces death of their silk worms, they, however, know that in the last period or age, every part of the laboratory should be opened; but often in avoiding one danger, they meet another, such as exposing the worms to cold and wind, which may harden them, and cause them to drop off at the moment they had begun to spin their cocoons; there is only the gentle and 
continual renewal and motion of the internal air that can be beneficial and natural to the silk worms.

It is considered astonishing, that one single worm which, when first hatched, only weighs the hundredth part of a grain, should consume, in about thirty days, above an ounce of leaves, that is to say, that it devours, in vegetable substance, about 60,000 times its primitive weight.

The result of experience tends to show that in warm climates, the silk worms consume rather less leaf than we have here stated, because it appears that the quality of the leaf is more nutritive. Count Dandolo says, that in the favorable regions of Dalmatia, he obtained one pound and a half of cocoons from fifteen pounds of leaves, and fifteen pounds of cocoons yielded one pound and a half of silk, although he adds that it was not so delicate and fine as Italian silk.

Facts relative to the increase and decrease of silk worms, in weight and size.

100 worms just hatched, weigh about

$1 \mathrm{gr}$.

66 after the first moulting,

1566

"after the second moulting,

66 after the third moulcing,

9466

"6 after the fourth moulting;, 40066 162866 On attaining the greatest size \& weight 9500 66 
Thus they have in thirty days increased 9500 times their primitive weight. The length of the silk worm when just hatched is about 1 line. After the first moulting its length is After the second moulting After the third moulting After the fourth moulting 66 466 $6 \quad 66$ $66 \quad 12 \quad 66$ $66 \quad 20 \quad 66$ After the fifth moulting many attain

the length of $40 \quad 66$

The length of the silk worm is thus increased forty times in twenty-eight days.

\section{Progressive Decrease.}

100 silk worms, when arrived at the highest state of maturity, size, and perfection, weigh

$7760 \mathrm{grs}$.

100 chrysalides, weigh

100 female moths, weigh 390066

100 male moths, weigh 2990 "6

100 female moths having deposited their eggs, weigh 170066

$980 \quad 66$

100 female moths dying naturally, after having laid their eggs, and nearly quite dried, weigh

$350 \quad 66$

In the space of twenty-eight days more, the silk worm has diminished or lost, thirty times its own weight. Its length, from the period of the largest growth until it changes into chrysalis, diminishes about two-fifths. 
During, and immediately after coupling, the moth appears to augment in weight; 100 female moths, which before coupling weighed 2990 grains, weigh immediately after, 3200 grains.

The worm diminishes gradually in weight during the last twenty-eight days of its existence; that is from the moment of attaining its perfection as a worm, until its death in the form of a moth, it eats nothing, is supported by its own substance, and yet accomplishes in that period, the most important functions of its life.

The facts we have stated demonstrate the strong vitality of the silk worm, and what pains and errors must be adopted to disease and kill it.

Remarks relative to the cocoon containing the healthy chrysalis, and those containing the decayed, gangrened or stained chrysalis.

When the cocoons are perfectly formed, they diminish in the first four days, three quarters per cent. each day; the other days the diminution is very trifling.

1000 ounces of perfect cocoons are composed, of living chrysalides, 842 oz. Of the remains or envelopes cast by the worms when they become chrysalides,

Of pure cocoons,

$$
\begin{array}{r}
4 \frac{1}{2} \quad 66 \\
153 \frac{1}{2} \quad 66
\end{array}
$$

$$
\text { Total, } \quad 1000
$$


Each healthy cocoon proceeding from a well managed laboratory contains the seventh part, and even 2.13ths of pure cocoon, when compared to the weight of the cocoon containing the chrysalis.

However, the fact is, that the average quantity of reeled silk obtained from the cocoon is about 1.12 th, that is to say that 140 ounces of perfect cocoons, with the healthy chrysalides, which contains about twenty-one ounces of pure cocoon, will only produce twelve ounces of spun silk.

Let us now connect the facts we have stated. About thirteen pounds seven ounces of mulberry leaves, will produce one pound of cocoons; seven and a half pounds of cocoons, containing the healthy chrysalides, will yield about eighteen ounces of pure cocoon; and these eighteen ounces of pure cocoons will give only eight ounces of spun silk.

The proportion then, between the weight of the mulberry leaves and that of the pure cocoon, is something about $895-9$ to 1 , and the proportion of the weight of the mulberry leaves and that of the spun silk is as $201 \frac{1}{2}$ to 1 . It is proper to add that the proportion between the spun silk drawn from the cocoon itself, may vary according to the ill or good management of the worms, and the skill of the reelers. 
The proportion between the weight of the cocoons containing the healthy chrysalis which can be spun, and that part called the coarse floss which cannot be spun in the same manner, is, on an average nineteen to one; that is to say, we find one pound of coarse floss to nineteen of cocoon that can be spun.

Cocoons containing the decayed, gangrened or stained chrysalides.

Generally, it is not possible to separate the chrysalides from those cocoons; the worm or chrysalides being turned into a corrupt, black, soapy substance, sticking to the inside of the cocoon. Sometimes the mummy is exceedingly black, and now and then detached, but most frequently it adheres to the cocoon.

Part of these cocoons may be spun; the stain does not always alter and spoil the silk, but the spinners can never be sure of the quantity of silk they may be able to wind from them, and they in general, dislike working on them.

The silk drawn from cocoons with diseased chrysalides, is never so fine as that given by perfect cocoons with healthy chrysalides; 860 cocoons of the diseased chrysalis weigh only a pound and a half; thus the cultivator loses twothirds, or three-fifths, upon this quality of $\mathrm{co}^{-}$ coon. 
We must here state, in speaking of these sorts of cocoons, that we have only made our experiments upon such cocoons as were brought to us from various places; therefore, our calculations may very possibly differ from those of other observers.

Facts relative to the production of the eggs.

Three hundred and sixty cocoons of the finest quality, weigh about 25 ounces. If we suppose half of these to be females, these will be about one hundred and eighty.

Each impregnated moth weighs about 32 grains, and altogether 5760 grains, which make about 10 ounces.

After four, five, or six days, each moth will have laid, on an average, 510 eggs. This number of eggs is equivalent to $7 \frac{1}{2}$ grains, as 68 eggs weigh a grain.

The 180 female moths consequently lay 91,8100 eggs, which weigh 1350 grains, or about two ounces and one-third.

This proportion of two ounces one-third per pound of cocoons, augments and diminishes, according as in the 360 cocoons which form one pound and a half, females predominate, and vice versa.

When the 180 moths have laid their eggs, they weigh only 1800 grains, and as it has been 
stated that the eggs weighed 1350 grains, it will appear that the moths have lost 2,610 grains, in earthy, liquid, and aeriform substance.

If the 91,800 eggs, obtained from 180 moths, yielded an equal number of silk worms, and that, well managed, they each, in time, formed a cocoon, from the eggs produced by the above mentioned $1 \frac{\mathrm{I}}{2} \mathrm{lb}$. of cocoons, we should obtain $382 \mathrm{lb} .8 \mathrm{oz}$. of cocoons, which the following year would yield eggs sufficient to produce $97,537 \mathrm{lb} .8 \mathrm{oz}$. of cocoons.

Facts relative to the buildings and utensits. -

To make the laboratory of the silk worms similar to their native climate, they must be enabled to live in it perfectly free from all moisture; the temperature should neither be too hot nor too cold, and, above all, they should never be exposed to sudden transitions from one state of atmosphere to another; the air should always circulate gently.

A warehouse, a cellar, or any other low, shady, close place, is best calculated for the preservation of the leaves during two or three days, provided it be cool, damp, and shut from air and light.

The utensils employed to rear silk worms, are constructed so as to spare time and expense, and for the better management of the silk worms 
and the eggs at all times. The interest of the cultivator, and the progress of the art will soon make the rules which have been laid down, very useful. 


\section{PAR TII.}

OF THE PREPARATION OF RAW SILK FOR EXPORTATION.

\section{CHAPTER I.}

PRELIMINARY OBSERVATIONS.

IT is not enough to plant mulberry trees, to raise silk worms and produce cocoons. The cocoons must be either disposed of by sale or employed to some profitable use. They can only be sold in the country where they are produced, because they will not bear the expense of transportation, and they cannot be exported beyond sea, because in the first place, they are a bulky article, and cannot be pressed, like cotton; the freight, therefore, and other expenses attending such transportation, would exceed their value, and secondly, they are subject to rotting in a sea voyage, because of the dampness of the sea air, and the decomposition of the chrysalis within. For these reasons, it is indispensable that the cocoons should find a ready market in the coun- 
try where they are produced. These markets are filatures, or reeling establishments.

Before silk can be employed in any kind of manufacture, it must be subjected to two different mechanical processes, which are reeling and throwing.

Reeling, or as it is also called spinning, is nothing else than extracting and winding off the silk from the cocoons, in threads of various thickness made up into skeins so as to fit it for exportation, or for sale in the markets at home. When it has undergone this process, it is called raw silk, and as such is in great demand among the manufacturers of Europe. It is performed by means of a cheap machine called a reel

Throwing is the name given in England, to what we call here twisting silk. It is performed like the twisting of cotton, by means of very complicated and expensive machinery. This part of the silk business is done in Europe by men who follow it exclusively as a trade or profession, they are called silk-throwsters. Several of them have already migrated from England into this country, and more will follow as soon as it will produce a sufficient quantity of ran silk to give them employment.

As this branch of the business does not belong to the silk culturist, we shall abstain from 
saying any thing upon it, any more than upon the operation of dyeing which follows it. These are trades by themselves, which have no connection with agriculture, and it is for agriculturists alone, and not for manufacturers that this work is designed. The reeling of silk is a proper employment for farmers' wives and daughters, either at home or in large filatures, therefore we think it necessary to give all the instruction in our power upon this important subject, besides that without the art of reeling, it is impossible to draw any profit from the cocoons, which, as we have said before, cannot either be exported or manufactured without being converted into raw silk, all the rest may be considered as more properly belonging to manufacturing establishments, and therefore is not within the purvieu of this treatise.

We are well aware that in this country, and particularly in Connecticut, the labor of silk culturists is principally, if not exclusively applied to the manufacturing of sewing silk. Fal from encouraging, we think it our duty to do all in our power to discourage this species of industry as it is now pursued, because we are fully convinced that it will never be a source of profit to the United States, but on the contrary it will check the progress of the silk culture. Bad habits, when they have once 
taken root in a country are not easily laid aside, and we regret to be obliged to say that agriculturists are of all men the most tenacious of their old customs, and that it is diflicult to introduce modern improvements among them either in their implements or in their method of husbandry. All travellers unite in making this observation, and this country, candor compels us to say, is not exempt from its application.

Every where in Europe, the converting of raw silk into sening silk, is the business of throwsters. The farmer confines himself to his cocoonery and his reel, and leaves the rest to those mechanics who have acquired a suficient skill in their various arts, and employ competent machinery. In this country, on the contrary, every thing, spinning, twisting and even dyeing, is done by the same hands, and with rude instruments not at all fitted for the purpose. It is with the common spinning wheels that silk is reeled from the cocoons and is twisted into an inferior sewing silk, which can never stand a competition with that imported from Europe. For near eighty years, this method has been pursued, and the art has not advanced a single step, yet every body knows that the great progress made in Europe in the various arts, is owing to the principle of the division of labor. 
But all this dear-bought experience seems to be disregarded. We have been repeatedly asked whether the same individual or the same company, could not plant mulberry trees, raise silk worms, wind the silk from the cocoons, throw or twist it, dye it in various colors, and manufacture it into different articles. We cannot qualify this gigantic scheme otherwise than by saying that if it should ever be tried, it will infallibly be found to be the ROAD TO RUIN.

Agriculture and manufactures are professions so entirely distinct, that they can never be pursued together with any expectation of success. It will be said, perhaps, that even the reeling of silk is a mechanical operation, and may be properly classed among the aris which belong to manufactures. This is undoubtedly true; nevertheless, it is one of those arts which the agriculturist may pursue with advantage. It may be assimilated to the spinning of flax, cotton and wool, and the weaving of common stuffs, the machinery of which is not costly, and which furnish an agreeable and profitable employment to the wives and daughters of farmers, in their leisure hours, and on that account are called domestic manufactures. But the throwing or twisting, and the dyeing of silk, do not come under this denomination, and must be left to those who profess those arts exclusively. It 
is true that reeling cannot be carried on to a great extent in farm houses, nor to the same advantage as in large filatures, but it is not on this account to be discouraged, because it is an useful and a profitable employment to the agriculturist, and employs many hands which would otherwise remain idle.

It must not be believed, however, that the art of reeling silk is as easy of acquisition as that of spinning flax, wool or cotton. It is a popular error not only in this country, but in Europe, that it requires neither instruction nor experience. This doctrine has been widely propagated in these United States by bold theorists who have never seen a regular filature, and with so much success, that the committee on agriculture at a late session of the Congress of the United States have reported it as a fact to the House of Representatives, that the art of reeling silk was sufficiently known, and therefore expressed it as their opinion that there was no need of instruction in that branch. This is a most lamentable error, as we shall show hereafter.

We have said that this false notion prevails not only in this country, but also in Europe. $\mathrm{Mr}$. Pitaro, an eminent writer on the silk culture, thus expresses himself in his excellent treatise, entitled, "La Science de la Setifere;" (on the 
science of the silk culture:) "Every body in Italy and France, say some ill informed theorists, knows how to reel silk from the cocoons, but the fact is otherwise ;" and he proceeds to show the falsity of this unfounded notion, in which opinion he is joined by all those who have knowingly written upon the subject, as will be shown in its place, by numerous extracts from their works.

But the manufacturers, who are sometimes compelled to employ silk thus badly reeled, are loud in their complaints. We shall expatiate more fully on this subject in another chapter.

We do not mean by saying this, to discourage the wives and daughters of American farmers. They will with proper instruction become perfect in this most important art. We know by experience that they learn much more quickly and shew more dexterity in the business than the females of Europe, whose hands are too much hardened by the labors of the field in which they are generally employed, and we think we may say with confidence, that a steady and sober American girl, who shall have spent a reasonable time in a regularly established filature, if she should marry an industrious farmer, will prove a treasure to her husband.

The art of reeling alone being so difficult as to require instruction and experience, we can- 
not but admire the enterprize of those American ladies, who, in their domestic manufacture of sewing silk, with only rude instruments, attempt to unite the art of the reeler to those of the throwster, and the silk dyer. We wonder, indeed, that they have attained such a degree of perfection in those arts, as they have done, but the perfection that is required to make silk a source of profit to their country, by those means they never can reach.

We hope to be permitted to expatiate somewhat at length on this domestic manufacture of sewing silk, because we consider it as the most pernicious system that could be introduced into a silk growing country, and fatal to the silk culture in the United States, as we shall be able to show by the consequences which it has already produced.

The principal seat of this domestic manufacture is the State of Connecticut, where it is mostly carried on in the counties of Windham and Tolland. In the year 1830, we took a journey to those counties, in company with Mr. Duponceau; it was in the season when the worms had finished their work, and the farmers were gathering their cocoons. We visited several cocooneries, and we must say that we were not satisfied with their method of silk culture. One of those silk culturists, who 
had read our essays on American silk, shrewdly observed that the author could not be a practical man, because he recommended sheltering the silk worm's eggs from the cold in the winter. "In this country," said he, "we leave our eggs exposed to the cold, and they nevertheless produce good crops of cocoons." We found the fact of the exposure of the eggs as he stated it; but we have no doubt, that a great many perished from cold during the winter, which would have made a large and valuable addition to the "crop of cocoons."

Our great object however, was to witness the process of the sewing silk manufacture, and to obtain correct information as to the manner in which that silk was disposed of, and the profits that arose from it. With that view, we remained several days in the town of Mansfield, where that manufacture is principally carried on. We saw the women at their work, which we need not describe here, as it is sufficiently known; we tried to persuade them that there were better methods of proceeding, but we found their prejudices too deeply rooted; they were well satisfied with what their mothers and their grandmothers had done before them. This is not peculiar to Connecticut; in the silk growing countries of Europe we have seen, women who reel their silk after a method 
taught by their great-grandmothers, and who will not adopt the modern improvements, to the great dissatisfaction of the silk manufacturers, who, of course, pay them an inferior price for their imperfect silk. But they do not mind that, and will still go on in their old way; human nature is the same all over the world.

Of this we are going to give a remarkable example. We found that in the town of Mansfield, there was an excellent Piedmont reel, which, it would seem, had been brought there as an object of curiosity, for no one made use of it. Desirous of convincing the people of the place of the advantage of using that instrument, and at the same time showing them how it might be employed, we gave notice that we would reel silk from it on a particular day, before all who should choose to be present. A room was prepared for the purpose, and a large concourse of people attended; cocoons were produced in abundance, which we reeled off, and the skeins were handed round to the company, and seemed to be much admired. Nevertheless, there did not appear to be any change in the popular opinion. One young lady alone, who had paid great attention to our method of proceeding, sent us in the evening a skein of silk, which she had reeled herself on the Piedmont reel. 
It was not, indeed, perfect, but it was sufficient to shew what American females can do, if they would only discard their too long indulged prejudices. 'That young lady with proper instruction, would have made an excellent reeler. What has become of the Piedmont reel since that time, we do not know.

The people, in general, looked upon us with a suspicious eye. They thought we were speculators who intended to take some advantage of them, and we found them shy in answering our questions; nevertheless, there were gentlemen amongst the most respectable class to whom we had been recommended, who freely and candidly gave us all the information we wanted. This is the substance of what we obtained from them, and from our own observation.

The domestic sewing silk, after being reeled, twisted and dyed, either by professed dyers or by the fair manufacturers themselves, is made up into skeins of certain dimensions, prescribed by the Legislature of the state, in order to prevent fraud and imposition, and is sent to the nearest store, where the merchant (as he is called,) receives it in payment for articles furnished to the family during the preceding year, or in advance for future supplies. He afterwards consigns it to certain hawkers or pedlars, who travel with it about the country, and dispose 
of it to the farmers in barter for the produce of the land, which finds its way into the merchant's store, who sells it to his neighbors on the credit of the next year's crop. The current price of that sewing silk was then four dollars a pound. It passed as a currency in that part of the country, the strongest proof that can be given of its poverty, althongh abounding in the richest production of the whole earth.

We were informed by persons the best acquainted with the business of the place, and worthy of full credit, that the sales of sewing silk in the two counties of Windham and Tolland, amounted annually to fifteen thousand or eighteen thousand dollars. That amount was produced by 8,000 pounds of raw silk, each made out of twenty pounds of cocoons, which makes in the whole, 160,000 pounds of those balls. * Now, we beg to be allowed a few observations on these important facts. .

In the first place, what a small sum is $\$ 18,000$ the highest in the computation, and that nominal ton, for the proceeds of 160,000 pounds of cocoons, to which is to be added all the labor and expense put upon that material to convert

* These facts were made public in an elaborate article on silk, in the American Quarterly Review for Decem. ber, 1831, and never have been contradicted, that we know of. 
it into sewing silk! Were the farmers to sell those cocoons at only 20 cents a pound, (the lowest price for good cocoons that we can think of, *) they would produce 32,000 dollars, nearly double the amount above mentioned and that in ready cash, (for they are a cash article) without the labor and expense attending the reeling, twisting and boiling, and the dyeing in various colors.

It may be said that since we were in Connecticut, that state has allowed to the reelers a bounty of fifty cents on every pound of raw silk reeled on an improved reel, which they should produce. We shall say nothing of the improverl reel, but we say that even with that bounty, which on 8,000 pounds of raw silk, which we have shown above to be their yearly production, would amount to only 4,000 dollars they would make less than by the sale of their cocoons; for 4,000 dollars added to 18,000 dollars, the admitted produce of their cocoons, wrought into sewing silk, make only 22,000 dollars, and their cocoons, at twenty cents per pound, would produce 32,000 dollars, difference 10,000 dollars.

If, on the contrary, the cocoons were sold

* At the experimental filature in Philadelphia, in 1830-2, forty cents were paid for every pound of cocoons that was offered; but that high price cannot be expected to continue. 
for realy cash, that money would circulate among the people, stimulate their exertions, increase the quantity of silk, and enrich the whole country; filatures would be established in their neighborhood, and the state of Connecticut, now so poor, would experience the highest degree of prosperity. We hope it is not too late, and that these fair prospects will yet come to be realized.

It might, perhaps, be enough to have shown that this domestic manufacture of sewing silk, after all the labor and expense bestowed upon it, produces in its results from 30 to 40 per eent. less than the first raw material (the cocoons,) would produce, if sold at a moderate price in an open market; but we think it will not be amiss to show here what are the causes of this enormous difference. We can easily demonstrate that it is entirely to be ascribed to the imperfection of the implements used, and of the methods pursued, all of which arise from a lamentable ignorance of the art of reeling and the mechanism of throwing, by which alone the raw material can be converted into good and merchantable sewing silk. $\Lambda$ few reflections on the facts above stated, will be suflicient to convince the reader of the truth of this assertion.

1. Twenty pounds of cocoons employed in making one pound of raw silk, are double the 
quantity employed in Europe for the same purpose.* Here then is a loss of one lundred per cent.

2. The sum of 18,000 dollars, which on the highest calculation is the annual proceeds of all the sewing silk made in the two counties above mentioned, at the current price of four dollars a pound, represents only 4500 pounds of sewing silk, and that is made, as above stated, out of 8000 pounds of raw silk; here is again a loss of near fifly per cent.

This loss is almost entirely produced by the imperfect reeling of the raw silk; in technical language it is called waste. We know that thrown silk loses something of its weight by the dissolution of the gum in boiling, but that never exceeds twenty per cent., and we know also that this loss is more or less compensated by the dyeing, particularly in black. In Europe, when sewing silk is dyed in black, the dyer is oblig. ed to return the same weight of silk that he has received; so that the greatest part, and sometimes the whole of this loss of weight, in Connecticut, must be attributed to waste occasioned by bad reeling.

The loss suffered in Europe on the best raw

* At the filature in Philadelphia, eight pounds of cocoons and sometimes less, were found sufficient for one pound of raw silk. 
silk, in the operation of throwing or twisting, seldom exceeds four per centum, when thrown in Italy, and five when thrown in England. (Badnall's view of the Silk Trade, page 63.) That which was sent to England, from the experimental filature at Philadelphia, to be manufactured, suffered only a loss of 35.8 ths per cent., which shows the great strength of American silk. It must be acknowledged that it was thrown with great care by Mr. Edward Molyneux, an eminent silk throwster at Manchester, from whose report we have stated the above fact.

Let this be compared with the immense loss suffered by the fair manufacturers of sewing silk in Connecticut. Waste of the cocoons in reeling, waste of the raw silk in twisting. Can the nation ever expect to derive profits from this mode of proceeding?

In vain Congress have tried to encourage that domestic manufacture by a duty, amounting almost to a prohibition, but the attempt has completely failed. In the year 1832, a duty not less than 40 per cent. was laid, by what is called the compromise act, on all imported sewing silk; the result has only been, nearly to double the price of Italian silk, and to encourage foreign manufacturers, as will be presently explained. In the year 1830, to which we have above referred, not an ounce of Connecticut 
sewing silk was purchased in our great sea ports. Since that time, notwithstanding the forty per cent. duty, this state of things has not changed; on the contrary, we are told, though we do not vouch for the fact, that the quantity of that kind of domestic silk has diminished; which shows that no improvements have been made in that manufacture. A few days ago we called on a respectable merchant of this city, who deals largely in silk and particularly in sewing silk, and asked to purchase some of the Connecticut manufacture; he told us that he had none, that two years ago a case containing about forty pounds of that article had been sent to him to be disposed of, but he could not sell a single skein, and he was at last obliged to send back the case with its contents, to the place from whence he had received it. He told us however, that he had a great deal of American sewing silk for sale, which he showed to us and which we found nearly equal to the imported. It was truly American, all except the material of which it was made. This requires some explanation, which we are going to give.

When in the year 1830, the silk bill came before Congress, and when afterwards American raw silk was sent from hence to England, it made a great noise in that country. The silk 
throwsters at that time were dissatisfied with an act of the British Parliament, which had considerably lowered the duty on foreign silks. Expecting to find employment, they came in shoals to this country. Several of them applied to us, whom we advised to return home, telling them that the country did not yet produce raw silk enough to employ them in throwing. A number of them, however, remained, and the silk bill having failed, they thought of some mode to turn their industry to account. They could not teach the art of reeling because they dıd not know it, England not being a silk producing country; the most of them, before they come over had never seen a cocoon in their lives; but they knew that England manufactured foreign silks, and that it was to her a great source of profit; they persuaded our capitalists to do the same. Those did not consider that if England had produced the raw material she would manufacture it in preference to that of other countries; they therefore imported raw silks from China and Bengal, and began at once, with the aid of the new comers, to enter upon the business of manufacturing silk. Sewing silk was their first object, it being the easiest to make, and of the most ready sale; besides that, it was protected by a duty of 40 per cent. This is the American sewing silk that was of- 
fered to us by the merchant of whom we have spoken. It was indeed manufactured in this country, but of foreign material, and under the name of American silk, it found a ready sale, at a price nearly equal to that of Italian silk, which in collsequence of the high duty has now risen from 6 to 11 dollars per pound; thus what was intended for the benefit of the agriculturists of the United States, has only turned to the advantage of the silk growers of Bengal and China, and the English throwsters who had made their way into this country. The politicians opposed to the tariff system, who by their opposition prevented the passage of the silk bill and who voted for the high duty on imported sewing silk, showed that their knowledge of the subject had not yet been improved by experience.

We consider this duty as a fatal blow to American agriculture: as long as we do not reel silk as perfectly as they do in Europe, as long as raw material shall lose from 30 to 40 per cent. in the throwing, it is evident that all those who wish to derive a profit from the silk business will prefer employing the foreign rather than the American material. Thus the farm. ers may plant mulberry trees, breed silk worms and produce cocoons, these will find no sale and no market any where, and a universal dis- 
couragement will put an end to that zeal for the silk culture which some go so far as to call a $m a$ nia, and which is now so prevalent in this country. The same thing happened once in Prussia, where, after many years of expectation, they were forced to cut down their mulberry trees, which they had planted and cultivated with great care. In Mexico, they make admirable sewing silk out of the foreign raw material, and with the aid of foreign manufacturers, and, although a species of silk worms is a native of their country, their government has in vain endeavored to turn their attention to the silk culture. It is much to be feared that the Americans at last will offer another sad example of the bad effects of giving encouragement to foreign industry in preference to their own.

There has never been a more striking example of the uncertainty of the protecting system. We do not wish to enter into the discussion of this long agitated question, but this we are free to say, that duties on imported articles have not seldom produced effects quite different from those that were intended. So little is the science of political economy yet understood. If we can venture an opinion, we think that the question will be solved by the general adoption of FREE TRADE.

What then is to be done under such circum- 
stances? We can only repeat what we have already said several times before, that without perfection in the art of reeling, no profit can ever be derived from the silk culture in this country.

The only preparation required to export silk to Europe, is the reeling. This, as we have said before, may be done by agriculturists, as well as the spinning of flax and other raw materials. For in fact, it is nothing but spinning, and is so denominated in Italy and France, where it is called filer, filare, which literally means to spin. Hence the word flature, which is applied to reeling establishments. Every thing beyond that, including the making of sewing silk, belongs exclusively to manufactures, and in no sense to agriculture, and, therefore, makes no part of this present treatise. 


\section{CHAPTER II.}

OF THE DIFFCULTY AND IMPOR'TANCE OF THE ART OF REELING.

We have said in the preceding chapter that it is a vulgar error, not only in this country, but also in Europe, that nothing is so easy as the reeling of silk from the cocoons, that the operation may be performed by any woman without instruction, or very little, like the spinning of flax or wool.

Nothing is so unfounded as this opinion. The delicate material which is here to be spun, in consequence of the extreme fineness of its fibres, is so difficult to be handled, and is liable in the handling to so many accidents, so much care and attention is required to draw and unite those almost imperceptible fibres into threads fit to be employed in manufactories, that it is impossible to learn this art without manual and ocular instruction, followed by practice and experience. We will endeavor in another chapter to describe, as clearly as possible, the various processes of this difficult art; but we are well convinced that our descriptions however minute, will not be sufficient without oc- 
ular demonstration, to enable any one to reel silk with the perfection that is required, and if a bad method is once fallen upon, it will be found almost impossible to eradicate it. The example of Connecticut, where, during a period of near eighty years, no improvement has taken place in the rude method which is pursued there, the glaring defects of which we have, we think, clearly demonstrated, is of itself sufficient to prove the truth of our assertion.

What mostly contributes to propagate and maintain this delusion in Europe, is that the farmer's wives and daughters are seen to reel silk at their farm houses, and having acquired great dexterity in it by long practice and experience, appear to perform their work with great ease. When Commodore Porter was at Mahon, a few years ago, and saw for the first time the women at their reels, he wrote to his friend Mr. Skinner, of Baltimore, that the process of reeling was simple and easy; afterwards, when better informed, he admitted that it required both skill and experience.

Another circumstance also contributes to maintain that false opinion, particularly in this country. Raw silk may appear beautiful to the unexperienced eye, and yet be full of imperfections. These causes have produced the vulgar notions that we are speaking of. But the 
manufacturers know better, as well as the authors who have written with a full knowledge of the subject. It will be well to quote here some of their remarks:

In the year 1828, the French government, with a view to encourage and protect their native production, had laid a heavy duty on imported raw silk, amounting nearly to a prohition. The consequence was, that the French reelers, having no longer any competition to fear, neglected their work and produced imperfect silk. Of this the manufacturers complained bitterly to the government, and successfully prayed for a repeal of the luckless duty, * as our farmers will probably pray, and we hope with like success, for the repeal of that of 40 per cent., on foreign sewing silk. They represented, that the imperfections in the reeling of the raw silk, occasioned a great increase of labor and expense to the manufacturer, and went so far as to say that their raw silk, not only that reeled in the farm houses, but in large filatures, with some honorable exceptions, had become inferior to that of Piedmont. 'Io remedy this

* Mémoire des Negociants de Lyon, au ministre du commerce et des manufactures, in the Library of the American Philosophical Society, Miscellanea, L. No, 384. It is mentioned in a report of the Committee of Agriculture, which will be found in the Appendix. 
evil, they prayed that the sum of 600,000 francs $(\$ 120,000$,$) should be appropriated by the go-$ vernment to introduce everywhere the most modern improvements in reeling, and particularly the machine of $\mathbf{M}$. Gensoul, of which we shall speak in a subsequent chapter; their prayer was immediately granted, and the money was distributed amongst the silk growing departments.

It would be too long to quote here all that those gentlemen say about the causes and consequences of bad reeling, and the loss which it occasions to the silk trade, it will be sufficient to cite their concluding sentence, which will afford to our readers an abundant subject for reflection: "Whatever," say they, "may be the skill of the French throwsters, it is impossible with raw silk imperfectly reeled, to produce thrown silk of good quality. The best method of throwing cannot give to that which is badly reeled the qualities that it wants, while, on the other hand, a bad method of throwing, does not deprive silk, which, being well reeled, is neat, equal, and nervous, of those indispensable qualities, for the good and easy fabrication of silk stuffs." - Memoire, p. 25.

Thus the operation of throwing or twisting silk after it is reeled, is thought to be less im. 
portant than that of reeling, on which every thing depends.

All the authors conversant with the subject, who have treated of the reeling of silk, have represented it as an art difficult to be acquired to the degree of perfection which is required by the manufacturers of Europe, and which is necessary to make the raw silk sell to advantage, and also as an art which is not to be learned in books, but which requires to be taught by manual and ocular demonstration. Let us be allowed to quote here some passages from a few of those writers:

Mr. Von 'Turken, an esteemed German writer on the silk culture, expresses himself thus: "6'The reeling of silk is of the highest importance, for thereupon depends the good quality and fitness for use of the raw silk, the goodness of the stuffs manufictured out of it, and the value of the silk in market. Even at the present time, from ignorance or negligence in reeling, great faults are sometimes committed; and it is proper to say, that when silk is badly recled, it can find no purchaser, or only at so low a price, that the culture of silk is not worth the while." -On the Sills Culture, p. 96.

The Chevalier Von Heintl, another German author, speaking on the art of recling, says: "6a 
mere description of this art, can never supply the place of ocular and practical instruction." -Unterricht im Seidenbane, p. 141.

Mr. De Teste, a Frenchman, who has written a valuable work on the silk trade, after giving a short deseription of the process of recling, expresses himself thus: "After reading this description, it will certainly be bclicved that the management of a filature is a very easy thing; if the theory is casy, there is nothing so diflicult as the practice." And further, he says: "silk is always 100 dear, when it is not well reeled."-De 'T'este, pp. 58, 59.

In another part of the same work, he says: "If it requires knowledge and particular attention and care to produce a good result from the rearing of silk worms, it is in the art of filature, that profound knowledge is required, and yet that art is too often abandoned to ignorance and routinc." - p. 12.

Mr. Pitaro, an Italian writer on the silk culture, of whose excellent work we have marle much use in the foregoing chapters, speaks very strongly upon this subject, and finds fault even with the directors of large filatures, for not paying a proper attention to their business. "Every body," says he "in Italy and France, according to some ill-informed theorists, knows how to reel silk from the cocoons, and yet the 
manufacturers in Italy as every where else, continually reproach the directors of filatures with receiving from them raw silks, which they can with difliculty make use of, and which they find diflicult to employ in the various tissues required of them. Besides the irregularity and inequality of the silk in strength and in size, their silk is very often crispy. But these directors, deaf to all observations, or wanting the necessary knowledge, take no notice of what is said to them; whence it follows, that their filatures can only degenerate, and the silk trade be destroyed."-La Science de la Setifere, p. 117.

The celebrated Count Dandolo, whose name is a host, also gives his testimony in favor of our position: "It is certainly," says he, " not easy to forsee to what sum the value of exported raw silk may rise, if the art of reeling shall become national, and be the object of the care and attention of intelligent, scientific, and patriotic individuals. Hitherto, this most valuable pursuit has only presented a mass of various and different methods, most of which were uncertain, and many of them absurd." He says further: "The art of spinning or winding the silk from the cocoons, is, as yet, entirely in the hands of people as ignorant as those who formerly reared silk worms; for instance, it is a well known fact, that of two spinners, spinning 
each seven and a half pounds of cocoons of the same quality, one will extract constantly eight ounces of silk, whilst the other will only draw six ounces, and perhaps still less." -Dandolo, l'art d'elever les vers a soie, pp. 383, 354.

The English writers do not say much upon the subject, the art of reeling not being practised in their country; their opinion however, on the necessity of good reeling is easily to be discovered in their works.

Of American writers we cannot quote many, for they almost all agree in the common prejudice that reeling is an art easily acquired; and some go so far as to say that we possess it in sufficient perfection in this country. There are, however some exceptions, and we are happy to avail ourselves of the testimony of the late William A. Vernon, Esq., of Rhode Island, in his excellent translation of M. De Labrousse's treatise on the culture of mulberry trees, which is accompanied witl valuable notes by the translator.

6" It is peculiarly," says Mr. Vernon, "from the manner of winding the thread from the cocoons, that depends the profit which the state derives from the raising of silk worms. Though we may know how to cultivate the mulberry, and to take care of the worms-though we possess the cocoons-all our cares are lost, 
if we bungle through the winding process." On the Cultivation of Mulberry Trees. Boston, 1828. p. 106.

Daniel Stebbins, Esq., of Northampton, in the state of Massachusetts, in a letter to the committee on Agriculture of the House of Representatives of the United States, dated February 10th, 1838, says in express terms, that "6 the quality and value of silk depend on the skill and perfection of reeling." - (Report of the committee, p. 19.)

We avail ourselves also with pleasure of the opinion of Mr. Lewis Tinelli, an Italian gentleman, who has been for several years an inhabitant of this country. In an interesting little work, entitled " Hints on the Cultivation of the Mulberry, with some General Observations on the Production of Silk," which he published at New York, in 1837, "I happened," says he, "to have an opportunity to see some specimens of raw silk and sewing silk, wrought by the ladies of Windham and Tolland, which show beyond doubt that the very best results would be attained if they could only adopt, positively and decisively, different processes and systems from those they now follow." He then proceeds at length, to show the necessity of establishing filatures where reelers may be instructed and their knowledge by this means 
disseminated throughout the country. We shall have occasion to refer further to what he says on this subject in a subsequent chapter.

Mr. Tinelli is a refugee from Austrian tyrany: he was one of the companions of Pellico, Maroncelli and Confalonieri, in the horrible dungeons of Spielberg; he has adopted this country, and resides now, as we understand, somewhere in the state of New York, we believe at Newburgh. We have been told that he was once a director of a filature, and from the knowledge displayed in the little work above cited, we are inclined to believe it. We have not the pleasure of a personal acquaintance with him.

In addition to these authorities, we have to adduce that of $\mathrm{Mr}$. Boucher, an eninent silk merchant and manufacturer at Paris, with whom Mr. Duponceau corresponded on this subject in the year 1831. That gentleman is of opinion that not less than ten years will be required to make our women perfect in the art of reeling, in which we differ widely from him. Reeling is performed in France by ignorant peasant girls, whose hands have become hardened by the labors of the field, in which they are constantly employed. Experience has convinced us that American women will acquire the art in a much shorter time. This correspondence be- 
ing very interesting and entering largely upon! the subject we have thought it too long to be inserted here, but it will be found in the appendix. Mr. Duponceau, not having kept copies of his letters, those of Mr. Boucher only will be given, with explanatory notes, as published in the Baltimore Chronicle of the Times, and afterwards in Hazard's liegister of Pennsylvania.

To these conclusive authorities many others might be added, but we think these will suffice. It is now ten years since we first recommended the exportation of raw silk to Europe, and yet not an ounce has been exported, except that that which was sent to England, France and Mexico, from our experimental filature in this city, though its success might well have induced the experiment. IJumerous improvements (so called) have been made in the reel and none in reeling. The machine has been improved by those who did not know how to use it. As to those pretended improvements our answer to the inventors has always been the same: " send your improved reels to Europe, and your fortune is made." But this advice has not yet been followed, at least that we know of.

The importance of the art of reeling is not less evident than its difficulty. We may plant mulberry trees, raise silk worms and produce cocoons to any extent; but the question still 
recurs, what shall we do with them? They must be employed in some manner or other, or else they will rot in the garret rooms of the farmer, and be a prey to the mice, the cockroaches, and innumerable insects. What then is to be done? Some say we must manufacture; but we cannot do that without knowing how to prepare the raw material, and the proof is that, as we have shown, our manufacturers are obliged to import their raw silk from foreign countries, because ours cannot be employed without considerable loss. Our silk, then, must be exported, until its increase in quantity, and its improvement in the preparation of it for the loom shall make it expedient for us to begin to covert it into stuffs. At any rate, whether we intend to manufacture or to export, the first thing to be done is tolearn how to reel. Without that we must eat our cocoons, as is said to be done in China, or suffer them to perish.

The necessity of exporting our raw silk is begining to be felt throughout the United States. It was distinctly admitted at the late great meeting of the friends of the silk culture in Baltimore, and at that which followed it at Philadelphia, and no doubt it will be so everywhere else.

We have said that the exportation which took place in the year 1830, from our experimental 
filature in this city, was sufliciently encouraging: to induce others to follow the example, though no one has ventured to imitate us. The silk we sent to England was native American silk, reeled by American women, in the first year of their apprenticeship, but under the direction and inspection of the author of this work; nevertheless, it could not be expected to be perfectly reeled, yet it was well received by the manufacturers of that country. In proof of this we give here an extract from a letter written by Edward Molyneux, Esq., of Manchester, whom we have already spoken of, to Petty Vaughan, Esq., Mr. Duponceau's friend and correspondent in London. It is dated the 21st of March, 1831.

66 I was prevented by illness from attending so promptly as I would have wished, to the samples of American silk which you committed to me; however, that is now done. I have put the silk through the different processes of winding, throwing and dyeing, and the result of all is highly satisfactory-so much so that I should think a fine field is open for the culture of that article on the other side of the Atlantic. There is no fault to be found with its quality, which is superior to India silk, generally, and, in my opinion, fully equal to that of Frioul or Trent. I have had it dyed black, the color 
which most tries the silk, but which it takes very well. Some improvement, however, must be made in the reeling.

"On the whole, I see no impediment to the growth of silk in America, and of its ready vent, in course of time, in this country. Of course a few years must elapse before our consumers get used to it, but this must eventnally be the case."

Our silk was not sent to England to be sold, but to be manufactured, as it in fact was, and produced beautiful stuffs. Before it was sent to Manchester to be thrown and manufactured, and before it had been tried at the throwsting mill, it was shown by Mr. Vaughan, to an eminent silk broker in London, to be estimated. The highest price of raw silk in England was then $22 s$. sterling, (\$4.84) a pound, and the lowest $9 \mathrm{~s}$. equal to $\$ 51.98$. Ours was estimated at from 13 to $16 s$., (from $\$ \$ 2.86$ to $\$ 3.52$, a very good price, for a beginning. The prices of raw silk were low at that time; they have since considerably increased, as will be shown presently.

Thus encouragement has not been wanting on the other side of the water. Our undertaking was much approved of in England, where raw silk is in constant demand. It was announced in Parliament by the member from 
Liverpool. Dr. Lardner, in his excellent treatise on the origin, \&c. of the Silk Manufacture, speaks of it in this manner, page 319 , note $\mathbf{K}$. London edition.

"The project of rearing silk worms in the United States of America, has very recently been renewed, and a small package of silk, the result of their attempt, was, early in the present year, (1831) imported into Liverpool.

"The President of the American Philosophical Socicty, established in Philadelphia, Mr. Duponcean, has, for some time, been desirous of encouraging this branch of rural economy, and lately established a filature under the direction of a gentleman, who having condurted a similar undertaking at Nimes in Francs is possessed of the requisite knowledge and experience.

"The quality of the silk hitherto produced in Pennsylvania is said fully to equal that of Bengal: it promises to stand well the various processes of dyeing; throwing and weaving; but as might be expected in the commencement of such an undertaking, the operation of reeling has not been conducted with the requisite degree of skill. The attempt has hitherto been made on too small a scale for the projector to form any satisfactory opinion upon the issue as regards its profitableness; and it yet remains to be seen whether the Philadelphians are in pos- 
session of facilities for this pursuit, which will counterbalance the high rate of wages prevalent throughout the state, and which would otherwise give a decided advantage in point of price to the raw silk of Italy and India, even in the markets of the United States."

It is not a little astonishing that our first essay-that American silk, reeled by raw hands from all sorts of cocoons, (for we purchased all that were offered, good or bad, even those brought by boys, who had fed their worms with rose leaves and the like,) should have met with such encouragement in England, the great mart for that kind of native produce. Nothing can be more encouraging if we consider it in a proper point of view.*

As we have already observed, the prices of raw silk have considerably increased within the last ten years. All the nations of Europe, from north to south, now turn their attention to the manufacture of silk; those whose country does not produce the raw material are obliged to purchase it. Hence arises the great demand, which can but go on increasing. By the last London prices current which have reached this country, (November, 1838,) we find that the

* We were also frequently interrupted by visitorg, so that we could not be constantly watching our reelers. 
best Italian silk, (Novi,) now sells in England at 33s. (\$7.26) a pound, and the least esteemed, that of Turkey, at 20s. (\$4.40.) The best China silk sells at $27 s$. (about 6 dollars.) The best Bengal, which the English call Novi, to assimilate it to that of a town in Italy, where the best raw silk is produced, brings, however, a less price than that of China, it sells for $21 s$. (\$4.62.) The two last mentioned silks are those from which is manufactured the pretended American sewing silk, which we have before mentioned. French raw silk is not mentioned in the London prices current, because the French do not export it. Their country does not even produce enough to supply their manufactures, and they are obliged to purchase a great deal of the foreign article. They purchase annually of it, according to Count Lasteyrie, to the amount of thirty millions of dollars. Dr. Lardner seems to fear that the high price of labor in this country will prove an obstacle to the production of raw silk. The Doctor is mistaken. It will, indeed, prove an obstacle, and a serious one to our silk manufactures, if we attempt them too soon, unless Congress will lay a duty of forty per cent. on all imported silk as they have on sewing silk; but as to raw silk, there is not the least danger.

In France female reelers, (and there are none 
others, ) are paid from thirty-five to forty sous, (American cents,) per day. At the Philadelphia filature they were well contented with twenty-five cents, and we might have had hundreds of them at that price. They were delighted with their occupation, which requires only attention, and their labor is performed in the open air, but sheltered from the rain and the too great effulgence of the rays of the sun, in the finest season of the year. They compared it with their work in the cotton factories, where they are confined, in the midst of noise, and without the advantage of fresh air. Girls, for turning the crank, may be had at a lower price. Besides, fuel is abundant in this country, which it is not in Europe. It may be added that raw silk is of little bulk, and will cost but little in transportation. These considerations, to which others might be adduced are suflicient to convince us, that the price of labor in this country will be no obstacle to the production of raw silk.

There is, therefore, no difficulty in the way, except the want of proper instruction in the art of reeling. It has been seen that there is a great difference in the price of raw silk, as reeled in different countries. It varies now in London from $\$ 7.26$, the price of the best Italian silk, to $\$ 4.40$, the price of Turkish silk, the 
least esteemed on account of its imperfect preparation. Have those who pretend that we have attained the perfection of that art, by the invention of new machinery or the improvement of the old, and by the superior skill of our reelers, considered this vast difference of near fifty per cent. between the raw silk of one country and that of another? The silk reeled at our experimental filature at Philadelphia, was only estimated at London, in 1831, at something like a middle price between the highest and lowest, and yet it was reeled under the superintendance of a professed teacher. What price do they suppose that silk reeled with their newly invented machines would produce in that capital? Why have they not tried it, to bring their assertions to this sure test? And if their improvements on the reel are so valuable, they must know that there are countries in Europe, where patents could be obtained, and high prices given for the patent right. Every body knows that there are consuls in this country from the European nations, on the watch for all improvements in the arts, who would not have failed to transmit an account of those wonderful machines, with the requisite drawings and models to their respective governments, who would have been glad to introduce them into the filatures of their country. But no- 
thing of the kind has been tried; no raw silk reeled by the improved methods has been sent to Europe to try the markets and test its value; and our.supposed improvements have remained unknown to the European world to this day. What would not France, for instance, have given for a reel so simple in its construction, that a girl, not over eight or ten years old, may, after a little practice, work at the reeling, admirably well, * or for the machine which is said to reel and throw silk at the same time? If such a thing be possible, and has been actually effected, (which we permit ourselves to doubt,) a large fortune surely awaits the ingenious inventor, should he know how to avail himself of his lucky invention.

Besides, if we wish to derive profit from our silk worms and cocoons, it is not sufficient that we should reel our silk tolerably well, so that it should command, if not an inferior price, at least not a higher one than that of 'Turkey; we should emulate the filatures of Novi and Fossombrone in Italy, and those of Nismes and Alais in France. Those have risen to their perfection by gradual improvements in the art

* Letter of Chauncey Stone, Esq., to the Committee of Congress on Agriculture, dated Burlington, New Jersey, February 1838.--Report of the Committce, April 20th, 1838, p. 25. 
and in the machinery; but there are men in this country who contend that the same can be done by machinery alone, without the aid of experience or of practical knowledge. .

Since the power of steam has been successfully applied to manufactures, navigation, and locomotion by land, and since the most ingenious machinery has been invented and improved in aid of that power, the world, dazzled by the effects which those truly admirable inventions and improvements have produccd, have been imbued with the opinion, that machinery moved by steam, or even without the aid of that powerful auxiliary, can perform everything, without the aid of human hands, or, at least, with as little of it as possible; we need not, therefore, have recourse to the long and patient experience of Europe, nothing is requisite but to invent new machines that will perform the work alone, propelled by the hand of a little boy, or a little girl, and the inventive genius of Americans has already been at work, and is said to have produced that effect! No one admires more than we do, the inventive genius of the people of this country. They have given too many proofs of it to the world to admit even the possibility of a doubt. But we will humbly contend that were every American a Franklin or a Fulton, they never will be able to effect 
this purpose. Nature has set bounds to the ingenuity of man, which he is not permitted to transgress. Machinery, however perfect, is an inanimate thing; it has no eyes, no ears, no senses by which it can direct its motions, or change them at pleasure, it must invariably follow the direction that is given to it, either by a first impulse, or the directing hand of man. This is in no case more strongly exemplified than in that of the silk reel.

The operation of reeling or spinning when applied to silk, consists of two distinet parts: 1st. The drawing of the silk from the cocoons; 2d. The winding it on the machine called the reel. This last part is performed almost entirely by the machinery, the first is exclusively done by the reeler, unaided by any kind of mechanical power. It is in this part that consists the dificulty of the operation, and we do not hesitate to say that this difficulty can never be removed by any invention or machinery whatsoever. It is physically impossible.

Those who have seen the females of this country draw silk from their cocoons to make their domestic sewing silk, fancy it very naturally to be one of the easiest things in the world, and to be hardly more difficult than the spinning of flax on the spinning wheel. They do not know in the first place, how much of the precious ma- 
terial is lost by their clumsy mode of operating, they do not know how much of it is to be lost in the subsequent operations, until it is manufactured into those elegant stuffs which cost this country from sixteen to seventeen millions of dollars per annum.* They think that sewing silk is the perfection of the silk manufacture, because they do not know that in Europe it is made of the silk of the refuse cocoons, which are not as susceptible of the perfection of reeling as the better ones. 'The thread used for sewing silk is a coarse thread, which will bear a strong pressure of the machinery in throwing, much more than the fine organzines and trams, on which the greatest profits are to be made. Those who think so are not to be blamed for their ignorance, since, as we have said before, it prevails eren in the silk growing and silk manufacturing countries of Europe.

But the making of sewing silk, or eren the reeling of raw silk for that purpose, is not the object that we mean to press upon this country. Compared to the others, it is trifling and inconsiderable. We wish to see the United States produce raw silk fit to be applied to the manu-

* In 1S35, silk was imported into the United States, to the amount of $16,59 \%, 983$ dollars $;$ in 1836 , to that of $25,0: 33,200$. Report of the Committee on Agriculture, 20th April, 183s, p. 1 . 
facture of those elegant stuffs we have spoken of, and to do it in the best, and, above all, in the cheapest manner, by saving the material as much as possible, without which no profits are to be expected. In this lies the great difficulty of the art of reeling, it consists in a good method of drawing the silk from the cocoons, which can be done only by skilful, dexterous and experienced human hands. With this the machinery has nothing to do, it does not assist the reeler in the least, and, we repeat it, no improvement can ever be made upon it, that will produce that effect.

The fibres of the cocoon are as fine as the finest human hair, and, of course, easily broken. They are not of the same degree of fineness through the whole ball; it is well known to naturalists that there are three layers of silk in the cocoon, the first or uppermost is formed of the best and strongest silk, the insect being then in full vigor and strength; after two or three days he becomes fatigued, his silk is thinner and less perfect, this forms the second layer; at last as he draws near his change into a chrysalis, he spins a still thinner silk, which gencrally falls with the chrysalis, to the bottom of the boiler, and sometimes in the best cocoons is entirely reeled off. Good reelers learn to distinguish those different layers by the sight and by the 
touch, while the cocoons are immersed in a basin of hot water; and in some filatures, where the best raw silk is to be obtained, these different layers of silk are separately reeled, being destined for different kinds of manufactures. This is only said by way of example; there are a number of other details, which no less require the skill and dexterity of the reeler, and are beyond the power of machinery.

As the perfection of raw silk principally depends on the equality of the threads, which must be of an equal fineness and strength through their whole length, that the weak parts may not be broken by the equal pressure of the throwing machine, which cannot be lessened or increased at pleasure ; it is necessary that those threads should consist, as nearly as possible, of an equal quantity of the delicate fibres of the cocoon. But the cocoons are not all of an equal size, and as we have said before the fineness of their silk varies; it follows, that to preserve the equality of the thread, they must be frequently changed and their numbers increased. or lessened; of this, the reeler must judge, and her fingers as well as her eyes direct her in this most delicate operation. Besides this, many accidents happen in reeling, which she must learn how to remedy with dexterity and skill; we shall only instance those entanglements of 
the silk threads, which are called marriages, which we shall explain hereafter, and which frequently happen from the nature of the operation. It would be too long to detail here all that is to be done by a skilful reeler; but we have said enough to show that no machinery whatever can supply the place of her experienced hand; and that she is not in the least aided by the machinery of the reel, which, as we have said, helps only to wind the threads which she draws from the cocoons, nor can it be improved to any other purpose.

Where machinery cannot help, steam cannot, since every body knows that it only serves to put machinery in motion. We are not ignorant that steam has been applied with success in England, to almost every kind of manufactures, even to the art of weaving. But silk has resisted their efforts. . The delicate material was injured by the rude and blind movement of the inanimate shuttle. The project, therefore, was laid aside, and we are convinced will never be resumed. We are speaking here only of weaving. In the operation of throwing, steam is successfully employed. 'That operation consists only in twisting. If the silk is well reeled, it is simple and easy, because the tender fibres of the material joined together by the reeler, have acquired sufficient strength to resist the pres- 
sure, and the thread is drawn by the force of the machine to the part of it on which it is wound, and is not as in reeling, directed to it by hand, after being formed with discrimination and care out of the fibres of the cocoons; if on the contrary, it be ill reeled, the operation of throwing is long, tedious and difficult, and sometimes impossible, because the threads, unequal in thickness, break at every minute, and the machinery must stop to reunite them, whence arises great loss of time and that loss of the material which is called waste. Hence arise complaints of the manufacturer, the low prices of ill reeled raw silks, or their entire rejection. The reputation of the country where the silk is reeled is also considerably injured.

The power of steam is also employed in large filatures, in the operation of reeling; but its use is confined to collateral objects; it supplies the basins of the reelers with pure distilled water, always of an equal temperature, which is very important in reeling. It enables them to change it at pleasure, so as to keep it clear from impurities. It lessens the expense of fuel, and is a very great saving of trouble and time, as the reelers are not obliged at every moment to attend to theirfires to maintain an equal heat; it also adds much to the brilliancy of the silk. Such are the effects of the admirable machine: 
of Mr. Gensoul, of which we shall give a description in another chapter, accompanied with a drawing. But it does in no manner dispense with the skill of the reeler, as will be seen when we shall hereafter minutely describe the process of that operation.

We have no doubt that when the art of reeling shall be sufficiently known and disseminated through this country, American ingenuity will find the means of further facilitating some parts of the labor and saving some part of the expense attending it, but until then we think that we must be content with learning and adopting the method pursued in those countries where the best raw silk is produced. 
C H A P T E R. III.

OF THE FILATURE SYSTEM AND ITS INTRODUCTION INTO THIS COUNTRY.

This system which is generally followed in Italy and France, has two objects in view:

1st. To provide constant and regular markets for cocoons, and thereby to give to the silk culture that encouragement and support without which it can never be profitably pursued.

2d. To secure and preserve the best method of reeling silk, and preparing it for exportation abroad on manufactures at home.

The means employed for effecting these purposes is the establishment of filatures.

Filature is a French word, which literally means spinning. When applied to raw silk, it is synonymous to the word reeling. It is also used to signify an establishment where reeling is performed. This word is not to be found in any English dictionary that we know of, because the English having no native silk, have no occasion for it. Dr. Lardner, however, makes use of it, and we believe some other modern English writers, who have treated of this subject. It has become familiar in this 
country, and we find it employed, not only in translations from foreign writers, and in original works on the silk culture, but in public documents, and in the Reports of Committees to the National and State Legislatures. We consider it, therefore, as a legitimate term, and would feel ourselves at a loss, were we to attempt to substitute another for it.

Filatures are of two kinds:

1st. Large or regular filatures.

2d. Domestic flatures.

Large filatures, are establishments in which from twenty to one hundred reels are kept at work. A greater number has been tried but found too dificult to be managed. Sometimes those establishments belong to merchants or manufacturers, but in general they are owned by individuals who follow no other business; they sell their raw silk to the merchants or to the manufacturers, who either export it or get it thrown and dyed, and employ it in their manufactures.

The owners of fllatures buy their cocoons from the farmers. They have agents who travel about the country, and such is the want of that article, that they purchase or contract for the crops even before they are gathered. Cocoons are brought to them from more distant parts, and never fail to be purchased. We have said 
that the French do not export their raw silk, although there is at present no law to prevent it; on the contrary, they buy large quantities of it, particularly from Italy; but the Italians, who manufacture but little, export their silk to different countries, and principally to England, where, from its excellent quality and the perfection of its reeling, it is held in the highest estimation.

At the head of every large filature there is a person to superintend the establishment, called a director, or overseer. It will be easily understood that the young girls at the reels are fonder of play than of work, and that if left to themselves, they will neglect their business and do their work in a careless and imperfect manner. But the profit of such an establishment depends on the quantity of silk reeled in a given space of time, and on the degree of perfection with which the work is performed. To unite these two advantages, it is indispensably necessary that the reelers should be watched and constantly attended to during the whole time that they are at work. The younger ones are to be taught, and their faults corrected, and the more experienced need also to be constantly overlooked. From this constant superintendence arises the perfection which is so 
much admired in the silks of Italy and France, and has raised their prices above all others.

Those directors must be skilled in every branch of the art; they are generally educated for that purpose and are well paid for their services. Their employment is sufficiently laborious. "A diligent director," says Mr. Tinelli,* "6 will promote a spirit of emulation among the women employed in large filatures, and bring them to an equality with their instructor. The attention of the superintendent is constantly given to see that every reeler, winds always the same number of cocoons on each reel, that the thickness of the thread be always equal, the silk always clean and freed from all refuse, and the water always of the same temperature, and the same clearness. Every morning the superintendent makes his inspection of each female reeler, as to the quantity of cocoons that each one has reeled, and the quantity of silk produced; not a single ounce can escape his vigilant supervision. Further-all, or the greater part of the skeins are examined and proved, in order to ascertain the titlet and the

* "Hints," p. 23.

†In order to ascertain whether raw silk reeled for organzine or tram of a certain number of cocoons, is really such as it pretends to be, a small skein of four hundred 
quality of the silk." Mr. Tinelli might have added many other things which are parts of the duties of the director of a filature, but which he did not, nor do we think necessary here to enumerate. We have said enough to show the importance of the office, and the labors that are attendant upon it.

Thus these large establishments are admirable schools for the instruction of young females, who after having spent there a sufficient time to learn the business, (during which time they are paid for their labor,) return to their homes, where they avail themselves of the knowledge they have acquired, set up small filatures, and reel silk for their own account. In process of time, these domestic filatures extend all over the country, to which they are at last an immense and neverfailing source of wealth.

Domestic filatures seldom consist of more than three reels, and sometimes of only one. They are worked on the farms by the farmers' wives and daughters, who have learned their art at a large filature; their expense to begin with is

turns of a machine, called a proof reel, is weighed, and the weight that it ought to have, being known, this is considered a sufficient test. The weight expressed in deniers, and doubled, is called the title of the silk, which is said to be of so many deniers. This is well known to all directors of filatures. 
trifling; they only need to have a reel and a basin, which are not very costly. There are some farms in France where the reeling is done by women who have not been taught in the large establishments, but their mothers and grandmothers were, at a time when the modern improvements had not been introduced: thus they follow the old routine and their silk is less perfect than that of the others; but the greatest number now consists of the pupils of the large filatures, who, being better instructed, of course produce the best silk.

It will be thought, perhaps, that the large filatures monopolize the whole business, and that there remains but very little for the domestic filatures to do. But this is not the case. We are assured by the merchants of Lyons in the memorial above cited, (page 24,) that one-half of the silk reeled in France is done in the domestic filatures. 'Thus they mutually aid and support each other as it is hoped will be the case in this country. In Piedmont, there is also a great number of domestic filatures, but they are kept under strict and severe regulations by the government, to secure the perfection of their work. We shall give hereafter, a translation of the ordinance or regulations to which all filatures are subject, not with the view that it should be imitated in this country, but in 
order to show what are consided in Europe as the principal requisites in the art of reeling. In France there are no such regulations, the reelers are left there to pursue their own interests in such manner as they think proper, as indeed must be the case in every free country.

It is expected that in the United States the greatest quantity of raw silk will be reeled in the domestic filatures; we are speaking here of the north, for in the south, it is probable that the rich planters will have large filatures on their estates, to be worked by their female slaves, but in the other parts of the Union, a few large filatures will be sufficient to instruct the young reelers, and domestic filatures will enrich the industrious farmers throughout the country.

The domestic reelers sell their raw silk in France to the manufacturers, and in Italy either to these or to the exporters, who are always ready to purchase it. It is paid for in cash, for a higher or a lower price, according to the quality of the silk, and the degree of perfection of the reeling. Good reelers scon acquire a reputation through the country.

Such is the system which is adopted in Europe, at least in those parts where the greatest profit is derived from the silk culture, and we do not know of a better one that we can recommend to this country. Large filatures are in- 
dispensable, and they must be placed under the care of competent directors. Otherwise, bad methods of reeling will be introduced and persevered in, until, at last, the farmer will be discouraged; he will neglect his silk worms, and end by cutting down his mulberry trees. If it be intended to make the culture of silk a source of wealth to the United States, they must be made sensible that the system of large filatures, is the only one that can produce that effect. We do not mean to exclude domestic filatures, we would, on the contrary, wish to see one on every farm, but without the instruction and support of the large establishments, we are fully convinced that they can never prosper.

If we should have convinced our readers of the necessity of introducing this system into the United States, the question then occurs, "how is that to be done?" Those who think that there is no art in extracting raw silk from the cocoons, that the machine does everything, and that the methods used in this country need not be improved, will find no difficulty in answering it; but we who think otherwise, find ourselves compelled to say, that "6the art of reeling must first be acquired."

Here a difficulty first presents itself, which we are far from considering as of little importance. We have said that the English cannot 
teach us this art, because it is unknown among them, and whatever some of them may pretend, it is impossible that they should know it, for this very simple reason,-that there being no silk worms reared nor cocoons produced in their country, they never have reeled themselves nor seen others reel, and therefore cannot be familiar with an art which can be acquired only by example and practice. It is certain that if this art were known to the English, it would greatly facilitate the acquisition of it in this country, as they speak the same language, and their habits, manners and customs do not materially differ from those of the United States. Intercourse would then be more easy, a greater confidence would be inspired, and every thing would be facilitated by the magic of a kindred race, a common language and common ideas, besides that, from the great intercourse that exists between the two countries, quacks and pretenders would be more easily detected. But this must not be even thought of. When an art is known only to foreigners, it is from foreigners alone that it ought to be learned.

The English, who understand their own interest as well as any nation that exists, are well convinced of this truth, and have acted upon it uniformly in circumstances precisely similar to this. Whenever they have wished to sup- 
ply themselves with raw silk from their colonies or distant possessions, they have begun by establishing large filatures and placing skilful and intelligent foreigners at the heads of them. About a century ago, being desirous of obtaining silk from their then newly settled colony of Georgia, they established a large filature in the town, now city of Savannah, and placed it under the direction of an Italian, named Joseph Ottolenghe. By this establishment an immediate spur was given to the silk culture; mulberry trees were planted and cocoons produced; and history tells us that the filature flourished and sent considerable quantities of fine raw silk to England, until the revolution, which put an end to it.

Mr. Ottolenghe, was a gentleman of education; his talents were not confined to the knowledge of his art. In the year 1771 he was elected a member of the American Philosophical Society; in 1782 we find his name in the list of subscribers in the 4 th vol. of the first Ameerican edition of '6 Blackstone's Conmentaries on the Laws of England," printed by Robert Bell, in this city; he is there described as "6 Joseph Ottolenghe, Esq., Superintendent of the Silk Culture, Georgia." This shows that a director of a filature is not, as some persons have believed, or affected to believe, a mere 
operative, a journeyman reeler, to be paid at the rate of one dollar per day for his manual labor. Of this the author can speak from experience.

In their East India possessions the British had pursued the same system; the East India Company have established one or more large filatures in Bengal, under the superintendence of European directors, from which they now import annually, 1,500,000 pounds of raw silk.*

Notwithstanding this, and all the attention and care which the company have devoted to the amelioration of their filatures, Mr. Badnall, in his "View of the Silk "Trade," $\uparrow$ tells us "the only sill calculated for the manufacture of the richest qualities of broad goods, is that produced in Italy, or in the southern provinces of France." "This requires some observations.

The silk of Bengal is not equal to that of this country. We have shown above, (page 226, that American silk was considered in England as equal to that of Frioul in Italy and superior to that of Bengal. Yet that silk, as we have also said, was made, (amidst numerous interruptions from visitors, ) of all sorts of cocoons, promiscuously reeled, and not fit specimens of

* "Lardner on Silk," pp. 70 and 73. London ed. †"View," p. 16. 
that branch of American produce. Therefore, we have reason to believe that when American silk comes to be properly selected and reeled, Mr. Badnall's observations will not be applied to it.

The English now import 1,500,000 pounds of raw silk from their filatures in Bengal, which at $\$ 3$ per lb., would make $\$ 4,500,000$. That is not enough for this country, when we consider that we pay annually to Europe from $\$ 16$ to $17,000,000$ for manufactured silk stuffs. But it must be considered, in the first place, that the business of producing raw silk is monopolized by the East India Company, and that the hands which they employ are those of the rude natives, who with dificulty abandon their own bad practices. "Bengal raw silk," says Lardner, (p. 73,) "is distinguished by two appellations-cointry wound, and filatures; the former being furnislied by native adventurers, who can employ none but the rudest methods for winding it; while the latter is produced by servants of the East India Company, and treated according to the most approved European methods."

In this country there is no danger of the business being monopolized. If our system be adopted, large filatures may be established in every State, and the production of raw silk will 
be proportionate. We have no rude ignorant natives to contend with; the American females are dexterous and intelligent-the imperfect methods at present used in Connecticut and elsewhere, will soon be abandoned, when it shall be found that the sale of the cocoons is far more profitable. Every thing, therefore, is in favor of these United States, and there are no bounds to the production of the valuable material.

We think we have said enough upon this part of our subject. If it should be admitted that in order to make silk profitable to this country, large filatures must be established, and foreigners employed to direct them, at least until the art is so well known, that we may be able to do without them, the next question will be, in what manner is this system to be introduced, and how are we to begin?

We do not write this book to flatter the American people, and to feed them with delusive hopes of large immediate profits. Therefore we have forborne all calculations of the probable produce of an acre of ground planted with mulberry trees, of so many ounces of silk worms' eggs, or pounds of cocoons, or of such a quantity of raw silk exported for sale. Enough of this has been done by other writers, and we do not wish to imitate them. These things depend on too many circumstances to be the subjects of exact calculation. We have already 
said, and we repeat it with full confidence, that the introduction of the filature system will prove in the end an immense and a never-failing source of riches to the United States. But it must be purchased by temporary sacrifices. No profits are to be expected in the beginning. Indeed, it is our firm opinion, that this system can but with great difficulty be begun, without the aid of the National, or of the State Legislatures. We do not say, however, that it is impossible. There is nothing that cannot be achieved by the spirit of enterprize, aided and supported by patriotism and perseverance.

Some political economists are of opinion, that every thing should be left to private enterprize and that it is alone adequate to the attainment of every useful object. "6'The aid of a government," say they; "6should never be required, and if required, ought never to be granted." Whenever they attempt to reason upon the subject, they never fail triumphantly to quote the answer of the French merchants to the minister Colbert, "Laissez nous faire;" (Let us alone.) This trite saying, serves them in lieu of an argument.

It is true that Monsieur Colbert, a minister of Louis XIV., who was a sincere friend to commerce and manufactures, and wished to promote them by all the means in his power, once 
asked the merchants what he could do for their advantage, and that the only answer he received was "Let us alone." But it is easy to understand the meaning of this answer, by recurring to the state of things in France at that time. At that day the French government was in the habit of vexing and harrassing the merchants and manufacturers by edicts and ordinances which they called Regulations, (Reglements, by which they attempted to regulate all the details of their business, even the length and breadth of the stuffs that they manufactured. Sometimes this was done from fiscal motives, in order to increase the revenue, but sometimes, also, it was the result of ignorance, and a busy intermeddling with things that they did not understand. The merchants were tired with those always troublesome and often injurious measures. They feared the government and its regulating system, and therefore they thought that the greatest favor they could obtain from the minister was that he should let them alone, and in this they acted wisely; but if they had had confidence in their government, they would not thus have rejected the offer of the minister, whose talents they had not yet experienced; for he was, in fact, a man of great abilities, he raised the commerce and manufactures of France to the highest degree of pros- 
perity, which the bigotted Louis afterwards disturbed and nearly destroyed by the persecution of the Protestants and the revocation of the edict of Nantz. In spite of let us alone, he laid out large sums of money for the introduction into France of improvements of the useful arts. The silk culture was the object of his particular care. It was he who purchased of an Italian named Benay, the valuable machine for the twisting of raw silk called the throstle, or throw. sting mill, which but ten years ago was entirely unknown in this country, and is now, as we have said, employed in twisting foreign instead of American silk. He not only paid Benay liberally for his machine, but gave him a pension for life and a patent of nobility.* Why did he not leave the silk manufacturers alone? Laissez nous faire!

In these enlightened times, the merchants of France no longer say to their goverument, let us alone; they do not, like the political economists we are speaking of, translate "Don't vex us," "Don't oppress us," into "Don't aid us," but they apply to the ministers for assistance, and do not apply in vain. Thus, in the year 1828, the merchants of Lyons, in the memorial we have so often quoted, requested the appro-

* Dictionaire Universel du Commerce, Verbo Organsin. 23 * 
priation of not less than 600,000 francs, (120,000 dollars) for introducing Gensoul's steam apparatus into all the filatures of France, and the author of this work was informed when in Paris last autumn, that their prayer was immediately granted, and that amount was distributed among all the silk growing departments, and this, not to introduce, but merely to improve the reeling of that material.

Neither do the states of this Union seem to care much for Laissez nous faire; for we find them everywhere by their legislative acts granting bounties for the planting of mulberry trees, the production of cocoons, and of raw, and even thrown silk. We beg to be allowed to make here a short digression, by some observations on this system of bounties.

As to the bounties for mulberry trees, we shall only say that the excitement now existing for the importation of those trees, particularly the multicaulis, the great quantity imported and the high prices given for them, would seem to render any other kind of encouragement useless. Nevertheless, we would not object to it, if connected with a good system of filature; for with filatures there can never be too many mulberry trees in this country. Without them, they may serve to feed the stoves and the chimneys in winter. 
As to cocoons, the best and only fit encouragement for that production is a good market. Without a market they must perish in the hands of the producer. And so they must, even if the highest bounties were given; for the farmer after receiving his bounty, will not be the better able to dispose of his produce by sale. The bounty which the law of Georgia allows is not less than fifty cents for every pound of cocoons; this is a higher price than can be expected to be given in any market. At that rate, the farmer may afford to burn his cocoons. In Connecticut, the bounty is fifteen cents, which is a tolerably good price. But after all, it will not help the sale of that article. We shall have plenty of cocoons, but no silk.

As to bounties for raw silk, we observe with surprise that no attention is paid, anywhere, to the degree of perfection with which the silk is reeled, but the bounty is to be paid for all raw silk exhibited, without distinction or difference. Thus it is the quantity and not the quality that is encouraged and rewarded, and thus haste and inattention are called to the aid of want of skill and knowledge to produce imperfect work. The greatest enemy to improvement in reeling could not have devised a better scheme to arrest its progress and to perpetuate the bad methods which unfortunately prevail. 
'This cannot have been the design of the wise and patriotic legislators who have proffered those bounties; but such will inevitably be their effect. It appears that they have imbibed the prejudices that too much prevail throughout this country, that raw silk is raw silk, however it may have been reeled, and that machinery can supply the want of skill. Need we then be astonished when we see an honorable committee of the House of Representatives of the United States, in their report to Congress above cited, express it as their positive opinion, "6 that the dull, tedious method of reeling by hand, which required a regular apprenticeship to learn, and years to acquire facility in the use of, has given way to the new patent reel, by which a person (even a child,) may learn in a few hours to reel, with great ease and expedition, a much more even thread than by the old process"? *

There never was, in our opinion, a more complete delusion. We hope that when we come to describe the operation of reeling, we shall be able to convince our readers, if they are not already convinced, that no machinery whatever, were it invented by Newton, Franklin, or Laplace, can ever supply the want of the skill and knowledge which that operation requires.

* Report of 20th April, 1838, p. 5. 
On the subject of bounties for thrown silk, we have little to say. We do not, as we have already said, consider silk throwing as at all connected with agriculture; it belongs exclusively to mechanics and manufacturers. The United States are not yet prepared for this branch of business, and we have shown that the premature introduction of it into this country, has resulted in the encouragement of foreign raw silk, to the great detriment of the native production of the silk worm and cocoons. Thrown silk cannot at present be made an object of foreign commerce. It will be much more profitable to export our silk in the raw state than with the additional cost of throwing, which could not be compensated by the profits of the sale abroad, besides that, it is in Europe subject to a duty, which raw silk is not. We cannot therefore, approve of the system of bounties applied to this article.

The necessity of the aid of governments to new and important undertakings, particularly to those on which the future welfare of the state in a great measure depends, is therefore admitted everywhere in practice, whatever it may be in theory. It matters not whether it be in the shape of bounties, of protecting duties, or of money appropriations, all interests have claimed it in their turn. Commerce and manufactures have had enough of it in the United 
States. It is now the turn of agriculture, the mother, the nurse, and the supporter of the two others.

Our eyes are naturally turned to the national government for the aid which the silk culture indispensably requires; but there is an objection in the way which, we fear, it will be difficult to get over. It is said that Congress has not the power to appropriate the public money to such a purpose, at least in the way that we would propose. Every body knows that in the year 1830, a bill was reported and recommended by the Committee on Agriculture to the House of Representatives, providing for the instruction of sixty young men in the art of reeling silk, so as to enable them to become directors of filatures. 'The author of this work was to have been their instructor. Thus, by employing one foreigner in the first instance, the dependence upon others would have been at an end forever after. The bill, after depending in Congress during three sessions, was ultimately rejected in 1832 , by a small majority. The principal ground of argument taken by the members opposed to it, was the unconstitutionatity of the measure. It was principally opposed by the Southern members, many of whom were afraid that it would result in a system of high duties upon foreign silks. 
On this question of unconstitutionality we are not competent to pronounce; but we may, perhaps, be allowed a few reflections.

In the month of May, 1826, on a report of the Committee on Agriculture, the House of Representatives of the United States, passed a resolution, authorizing the Secretary of the Treasury, (who was then the Hon. Richard Rush,) to have prepared "6 a manual on the growth and manufacture of Silk." The Secretary employed a gentleman of Philadelphia to compile that manual, which he did in a volume of rising two hundred pages, and was, we presume, liberally paid for his labor, in which he appears to have been employed upwards of eighteen months, for it was only in May 1828 that the manual was presented to Congress, who ordered six thousand extra copies of it to be printed, not for the use of the members, but, as is stated in the "Report of the Committee on Agriculture," of the 20th of April, 1828, to which we have so often referred, for distribution, * by which we understand, distribution among the people for general instruction.

Here, then, are two sums of money (no matter what the amount was) paid out of the public funds, one to the author of the manual, the

* "Report," p. 5. 
other to the printer of the six thousand copies for the instruction of the people.

Now we would ask, in all humility, what is the difference between the appropriation or payment of these two sums for the instruction of the people in matters relating to the growth and manufacture of silk, and that of 40,000 dollars, which, by the silk bill above mentioned, and which was deemed to be unconstitutional, was to have been applied to their instruction in the art of rce!ing and preparing silk for exportation. In both cases, the money was, or was to be applied to the purchase of knonledge. We are at a loss to know why that knowledge might be constitutionally purchased in the shape of a bock, and not in any other form?

Will it be said that in one case the sum was comparatively small, and in the other more considerable? But it seems to us that the question does not turn upon the amount, but upon the principle. 'The appropriation of one dollar, if not warranted by the constitution, seems to us to be as much a breach of that compact, as that of one million.

Must we then conclude that Congress, in purchasing silk manuals to diffuse instruction among the people, violated the constitution of the United States? We indignantly repel this suppasition; but we are not so sure that if they 
had passed the silk bill, they would have been guilty of a breach of the Federal compact.

We do not profess to be learned in the constitutional law of the United States; we can only call to our aid our plain unsophisticated common sense, when we attempt to explain its text. For instance, we might, perhaps, say that by that instrument, the power of regulating commerce with foreign nations is given to Congress in express terms. We might ask whether the exportation of raw silk to foreign countries, is not a branch of that commerce, which Congress is authorized to regulate? And whether precautionary regulations to prevent fraud and imposition upon foreigners, and to give a sure and a permanent value to that branch of commerce, are not within the limits of their authority? But we think we have said enough upon a subject on which we do not think ourselves competent to decide.

'The fate of the silk bill is truly remarkable. In 1830 and ' 31 , it is received by Congress with apparent enthusiasm. In 1832, it is reported to the House with a few amendments by the Committee of the Whole, and the next day it is suddenly rejected in the same House by a small majority, as it would seem, on the ground of unconstitutionality, though it is not certain that it was negatived on that principle. In 
1838 , unconstitutionality is no longer spoken of; the Conmittee on $A$ griculture, on the contrary, in an elaborate report, tell Congress that at the time when that bill was proposed, and with the lights then possessed, it was 66 wise, prudent, and important"; but that "the subsequent ingenuity and experience of the American people, render it unnecessary. " *

We have sought in vain for the proofs of that increased knowledge and experience. We have looked in vain into the London prices current, to discover the prices at which American raw silks were quoted. They are not even named. At home, we have seen nothing but sewing silk sold as American, but made of the foreign raw material. We must acknowledge that we do not perceive that improvement, which is thought to require no aid, either from the government or from any other source.

We are still of opinion, that unless there be in the constitution an insurmountable obstacle, the national government is the authority to be looked to in the first instance; because the object to be attained is not only a perfect but an uniform method of preparing the raw silk of this country, whether for exportation or manufacture, and to prevent, as much as possible, the mono-

* Report, p. 6. 
poly of the business by states or by individuals. It is necessary, also, to give a general impulse to the silk culture throughout the Union.

If, however, the general government, for reasons that we cannot foresee, should decline giving its support to this most important and truly national object, the next to be looked to, will be the Legislatures of the different states.

Here we must not expect the sameadvantages that would have resulted from the action of the general government. If the silk bill had passed in the form in which it was proposed, we should be no longer under the necessity of employing foreigners. Sixty young Americans would now be fit to be employed as directors of filatures. There would be enough to supply the capitalists in the north, and the planters in the south. Their system, their method, and mode of proceeding would be the same. There would be no difference between the raw silk of Boston and that of New Orleans. But it cannot now be the case. One state may employ a Frenchman, another an Italian, another, perhaps, a Chinese. One may employ a skilled director, another a quack or a pretender, and these will not fail to present themselves; several of them have already appeared, and some states have been led by them into measures that have had no success. The silk 
bill, by employing one well recommended foreigner for two years, was intended to prevent the necessity of having recourse to them forever after. Its failure, therefore, is justly to be regretted.

But this is not all. The states will naturally act for their own interest, and not think themselves bound to provide reelers or directors of filatures for the whole Union. They will cause no more to be instructed than they will want for their own filatures; the foreign director, himself, will not be very much disposed to communicate his art, except to the female reelers, who cannot prove to him dangerous rivals; that art may and will, it is true, gradualiy make its way through different parts of the country; but the progress will be slow, and the success various; in the mean time, the state will acquire a reputation abroad for the perfection of its raw silk, and it will be difficult to draw the business from that channel. Happy the state that will first begin!

But the states may not be sensible of their interest, or will be unwilling to lay out the money that the first experiments will require. The business will then necessarily fall into the hands of companies or of individual capitalists. Here a narrower system must be expected to be pursued; monopoly will be the aim of every 
one, and the knowledge of the art will be still more concentered in a small number of persons. Every one will work for himself; foreign pretenders will abound in the country, uniformity in the process of reeling must not be thought of, and every thing will be done at a venture. Nevertheless, some filatures, like those of Novi and Fossombrone in Italy, and Nismes and Alais in France, will, in time, acquire a high reputation abroad, and the others will do as well as they can. Upon the whole, whether the thing be first begun or set on foot by the government of the United States, by that of the particular states, or by individuals, we do not hesitate to say that the system of filatures, in whatever manner it may be introduced into this country, is the only one by which it may expect to derive profit from the silk culture.

We cannot conclude this chapter better than by inserting here some part of the introduction of Mr. Pitaro to the third part of his excellent work on the silk culture, in which he treats, much too briefly, of the art of reeling silk from the cocoons. It will enforce what we have said on the importance and the dificulty of that art. "We have already," says the celebrated author, "completed the greatest part. of our labors, we have shown how the silk culture might be carried on with the greatest success, $24 *$ 
but of what avail will be a multitude of cocoons, if we do not know how to extract the silk from them, with the intelligence, economy, and skill which the various kinds of manufactures require? For what purpose shall I have written the above treatise, if, contented with raising silk worms and producing cocoons, you do not know how to unwind these with skill, care and economy, to draw and join together into even threads the delicate fibres, and to do it with the perfection required by the silk manufacturers? It is only when the silk possesses the qualities and proportions required for the different tissues, that the reelers are applauded for their labor, and both the seller and the purchaser find their profit in it. Laborious and intelligent men know how to acquire wealth, the indolent, on the contrary, the enemies of mankind, the apologists of falsehood, those stupid beings, who only know how to waste the productions of the earth, aided by the skill and ingenuity of man; those, we say, will only reap poverty and disgrace, and contempt will be their portion."

Thus far Mr. Pitaro. We will now, like him, proceed to the practical part of our subject.

Filatures! filatures! that is the constant burden of our song. 


\section{CHAPTER IV.}

OF FILATURES, THEIR EXTERIOR FORM AND INTERNAL ARRANGEMENTS AND OTHER MATTERS RELATING TO THEM.

We have already explained the origin and various meanings of the word flature; we have shown the difference between the large establishments called by that name, and those which are denominated domestic, and which are often confined to the management of one single reel; we have also made known the nature of the office and the various duties of a director, where the number of reels requires that such a person should be employed; we shall not, therefore, repeat what we have said upon these subjects.

The object of the present chapter is principally confined to the place and the buildings in which large filatures are to be carried on: to their external disposition and internal arrangements. Much of what we shall say on these subjects, will apply also to domestic filatures, and the differences that may exist shall be properly noticed. It must not be forgotten that it is from large filatures only that we may expect to have well instructed and skilful domestic reelers, and therefore that those important es- 
tablishments require our first and our most particular attention.

The buildings or erections in which the reeling of silk is performed are not exactly on the same plan in Italy and France, at least in the parts of those countries that we have seen. In the north of Italy those buildings are walled on all sides.* They are in the form of an oblong square, two stories high: on the ground floor are the reels and the reelers, above are kept the cocoons, with the spare reels and other apparatus not in immediate use.

An American gentleman, $\dagger$ who was at $\mathrm{Sa}$ vannah thirty-five years ago, when the filature erected there by the British, under the direction of $\mathbf{M}$. Ottolenghe, and, of course, on the Italian model, was still standing, though it was no longer used for its original purpose, but was a ball or assembly room, and sometimes served as a place of worship, has been kind enough to favour us with a description of that building, as far as his memory could serve him. It was a two story building, about sixty feet in

* Dr. Lardner, in his valuable work on the origin, \&c. of the silk manufacture, (page 182, London edition,) says, that a building designed for a filature, should be open on one side and walled on the other. We have never seen any of that description.

† William Ladd, Esq., of the state of Maine. 
length, and thirty feet wide; the roof projected slanting on all sides, to protect it against the rain. From this description we presume that it must have held from twenty to twenty-four reels, at least it might have contained so many with ease, (ten or twelve on each side, but only twenty if a part of the room was reserved for the director, and the women and children employed in sorting the cocoons, taking off the floss, and other accessory or preparatory matters, which could not be done by the reelers without interrupting their work. We do not, because the room could contain so many reels actually employed, necessarily presume that there were so many used, because that must have depended on the quantity of cocoons with which the filature was supplied.

We find recorded in history, that in the three first years of that establishment, that is, in the third year, there was only eight pounds of raw silk exported to England, which were thrown and manufactured there, and a dress made for the Queen, who was graciously pleased to wear it in public. This small quantity is not astonishing; the colony had just begun to be settled, the mulberry trees had hardly had time to bear a sufficient quantity of leaves, and the inhabitants, then, must have been very few in number. We learn from the same source, 
that in the course of seventeen years, (from 1755 to 1782 ,) there were exported to England 8829 pounds of raw silk, which, on an average, makes a little more than 500 pounds a year. This will appear very little when compared with the quantities of raw silk annually produced in filatures of the same size in Europe; but when we consider how small was the population of Georgia at that time, we ought rather to wonder at their having done so much. It is probable that in the first years much less, and in the last much more than the average quantity above mentioned, was reeled in that establishment. It is evident that if the Revolution had not intervened and put an end to the silk culture and to the reeling and exportation of raw silk in that colony, Georgia would be, at this time, a great and a rich silk growing country. Their example is well calculated to encourage us; if they did so much with such limited means, what cannot be expected from the United States in their present condition, with a numerous population and large capitals, their numbers and prosperity increasing every year, they must soon become, as we have often predicted before, a rich silk growing and silk exporting, and, at last, a rich silk manufacturing country.

The French filatures are on a different plan 
from those of Italy. Like them they are in the form of an oblong square, but open on all sides, and surrounded only by a low wall of masonry, about four feet or four feet and a half high, with openings at convenient distances for passages. The roof, which projects, as in the Italian buildings, is supported by strong, square, stone pillars or columns. Thus the air is at all times freely admitted, which is of great importance to good reeling. The building has two stories, both very lofty. In the upper story, the provision of cocoons is laid on shelves, of which there are several tiers, above one another. In the winter, the building is closed by wooden sliders; in the reeling season, there are glazed sliding panes, which may be raised or let down at pleasure, and serve to guard against the rain in case of sudden gusts ; for no reeling is performed in damp or rainy weather. There are no cellars; the fuel is generally kept in an adjoining or neighboring tenement, or under a shed. The buildings are commonly on the estate of the owner, in the country or in the suburbs of a town, and not far from his mansion, that he may the more easily attend to what is doing there.

We will not undertake to decide on the respective merits of those two modes of erecting filatures. Perhaps the climate or the tempe- 
rature of the country, may require the adoption of the one in preference to the other. What is certain is that silk is equally well reeled in Italy and in France.

The things that are indispensably required in a filature, are the free admission of light and dry air, and to be guarded against sudden changes in the weather. In this country, where the temperature is so variable, such sudden changes are particularly to be feared and guarded against.

According to Mr. Pitaro, the best exposure for a filature is north and south, as the clearest light is thereby obtained. The next best is south east and south west. It will be easily understood that we speak of the exposure of the long sides.

Whatever be the length of a filature, its breadth should be such, that reels may be placed on each side with convenient intervals and passages. The Piedmont reel is six feet four inches long, and two feet $1 \frac{3}{4}$ inch wide. It should be placed with the great wheel or hasp, towards the wall. A passage of two feet should be left between each reel, a small passage of eighteen inches between the wall and each row of reels. These passages being only for convenience, they may be increased or diminished according to the size of the filature. 
A space should be left at one end, for the accommodation of the women and children who sort the cocoons, strip them of their floss, \&c., and if possible, a small railed place for the director to sit in, with a table for him to write on and take notes of the quantity of silk reeled, \&c. These conveniences add much to the facility of the business.

Our experimental filature in 1830, and ' 31 , was under a temporary shed, erected for that purpose, at the end of a lot about 22 feet wide, adjoining the house No. 148, in Pine street, between Fifth and Sixth streets in this city. It contained ten reels, seven of which were placed breadthwise, on one side, and three lengthwise on the other. It had no upper story. It was guarded against the rain and the too great effulgence of the sun's rays by a wooden slider which might be raised or let down at pleasure. It might have been more convenient, but we aimed at economy, and had not sufficient room. We wanted a place in which to keep the cocoons, which we had to bring from a distance, and it occasioned a great loss of time.

We think that a large filature with all its conveniences should not be of less than twenty reels. Mr. Pitaro speaks of a filature of fourteen reels with a steam apparatus; we have never seen any of less than twenty; this, how- 
ever, is only a question of expense, of which every one can judge for himself.

We have not had an opportunity to see domestic filatures in Italy; we have only seen the large filatures at Nori, therefore we can say nothing of the manner in which the farmers proceed in that country. In France they generally reel under sheds, which, in winter, serve them for other purposes, If good merchantable raw silk is wished to be produced, reeling should never be done in a close room: of course never in winter. It may be done in the proper season in a well aired and well lighted room on the ground hoor. At Nismes, and other towns in France, where there are large filatures, when good reelers leave the establishment by getting married or otherwise, the owners give them every day a certain quantity of cocoons to be reeled off, which they do at their own houses. The owner finds the reel, the basin, the cocoons and the coals. They are paid by the day and not by the job. As they are known to be excellent reelers, the quantity of silk reeled is not so much looked to as the quality. They generally do the work in a lower room of their houses, well aired and lighted.

We shall not describe here the machinery, apparatus, and various implements employed in a filature for the purpose of reeling; we will treat of them hereafter. 


\section{CHAPTER V.}

OF THE PREPARATIONS FOR REELING.

These consist of two things:

1st. Taking off the floss from the cocoons.

2d. Sorting and separating the cocoons.

When cocoons are brought to market or are purchased from the farmers or growers at their houses, they are generally, and we might say always sold with the floss on, the farmers only pick it out when they intend to reel the silk themselves; it is therefore necessary, before the cocoons are brought to the boiler to be reeled off; that they should be carefully stripped of the floss or tow silk in which they are enveloped. 'This is an easy task, and may be performed by boys or girls. The cocoons should be handled gently, so as not to flatten or bruise them, which would be very hurtful, and the floss must be picked off from them so as to leave them perfectly clean. The floss should not be thrown away, but carefully gathered and kept for sale or for use. We shall show hereafter in what manner it is to be employed.

The separation or sorting of the cocoons requires particular attention. 
Among a mass of cocoons there are some which cannot be reeled without much difficulty, and others which cannot be reeled at all. There are also some the silk of which is preferred in the markets of Europe to that of others, and commands a higher price. It is, therefore, necessary to sort them before reeling, and keep them separate.

The principal differences between cocoons, are in their color and their quality. Another difference has been suggested by some foreign writers; it is that between the soils and climates where the silk worms have been reared. This difference cannot be of any great consequence in Europe, where the eggs and the cocoons generally originate in the same place or district, at least in the same country where the silk is reeled. But in the vast territory of the United States, there is such a variety of climates, that this difference may not be without importance. Ten pounds of cocoons are required in Europe to make a pound of raw silk; in Pennsylvania, eight pounds have been found sufficient. We have even drawn one pound of silk out of much smaller quantities of cocoons, particularly from South Carolina and Louisiana, but as these were only experiments on a very small scale, we abstain from giving particulars, which, perhaps, would not be easily believed, and at 
any rate cannot be considered as general rules. Further experiments will decide how far it is necessary that cocoons from different states should be separated from each other before reeling. We will at present only speak of their differences of color and quality.

\section{1st. Of color.}

The principal colors of cocoons are white and yellow. The white is everywhere preferred, and the silk of white cocoons, when well reeled, commands the highest price. We cannot too strongly recommend preserving only white cocoons for reproduction; by that means all other colors would in time disappear.

There are two shades of yellow cocoons, the deep and the pale yellow. The latter are preferred. In most filatures, however in Europe, they are reeled together. There is a third species, which is rare in Europe, but appears common in this country, at least we have had many of them in our experimental filature, and reeled them separately. They are of a pale yellow, strongly tinged with light green; we therefore called them green cocoons. In Europe we have always seen them reeled with yellow cocoons. In the boiler they change to a dusky white.

But there is another species of cocoons which $25 *$ 
we have never seen or heard of anywhere but in the United States. They come from Mississippi and Alabama. They are of a dirty brown inclining to red, which made us call them, at first mud colored, afterwards, however, we changed their name to coffee colored, as they are of the color of ground coffee. Under that name, we sent silk reeled from them to England and France. In the latter country no observation was made on them; of the green cocoons it was said that the specimens we sent, reeled off very well, though they were pointed at the ends. Mr. Molyneux, our throwster at Manchester, wrote of the two last mentioned species of cocoons, as follows, "the straw, (he meant the green,) and coffee colors take the dye as well as the yellow and white; still it must be remembered that a prejudice will naturally exist for some time in favor of the latter ;" from which we infer that the silk of those two species of cocoons was not known in England in 1831, when Mr. Molyneux wrote. We leave to future experiments to decide whether these two last colors, the coffee color, particularly, are to be separately reeled, or whether, as in Europe, the white and yellow only should be kept separate from each other.

\section{2d. Of quality.}

The first division of cocoons, in respect to 
their quality, is into good and bad. The good cocoons are those which can be reeled without any extraordinary trouble. 'These are divided into two classes, the first and the second, which good reelers will keep separate from each other, because the silk that they respectively produce, is not equally valuable.

The first class of good cocoons is known by their firmness and hardness and the fineness of their texture, the second class has less of those qualities, which an experienced reeler will easily discern. We have not recommended this separation to the farmers, who reel their cocoons themselves, because it is only done in great filatures where the very best silk is to be produced.

The bad cocoons are of two kinds, those which cannot be reeled at all, and those which may be reeled, but with more or less difficulty, so that they should not be mixed in reeling with the good cocoons.

The cocoons which cannot be reeled, are:

1st. The perforated cocoons, that is to say, those from which the moth has escaped, leaving a hole that interrupts the continuity of the thread.

2d. The thin cocoons, the texture of which is loose, and yields to the slightest pressure. Sometimes the texture of these cocoons is so 
loose, that they are transparent, and are, to a good cocoon like gauze to satin. These are spongy, and when by negligence or inadvertence they happen to be put into the boiler with good cocoons, they imbibe the water and fall to the bottom. They must then be taken out, when discovered, and put aside, to be afterwards dealt with as waste silk.

The bad cocoons which can be reeled, are of various kinds; they are reeled with much difficulty, and never so perfectly as the good ones, therefore, their silk is only employed in the manufacture of coarse articles. They are:

1st. The double cocoons, called dupions, of which we have spoken above, (p. 118.) It is clear that their silk often intertwines, and therefore breaks in reeling, which gives a great deal of trouble to the reeler. It is because of this extraordinary difficulty, that we have recommended to the farmers who reel their silk themselves, to separate these from the other bad cocoons; in large filatures the experience of the reelers makes this unnecessary.

2d. Spotted cocoons, that is to say, those in which the chrysalis has died from some sickness, so that by its bursting or otherwise, the silk has become soiled, and the cocoons covered with black or colored spots.

3d. Cocoons that are otherwise defective, ill- 
shaped, or imperfectly formed, so that it may be easily seen that they cannot be reeled off freely. It is sometimes the case with those that are pointed at one end, but it is not so generally; the point must be very sharp to place them among the bad cocoons, and we do not think that it should be often done. We have seen pointed cocoons in general reel off very well.

4th. 'The cocoons called sattiny. They are of a coarse tissue, furzy like flannel, and the surface shines. We have seen some, but not many in this country, neither are they numerous in Europe.

The French writers have given names to the different sorts of cocoons. They talk of Chiques, Choquettes, Cocalons, Soufflons, and a variety of other denominations, which in our opinion only serve to perplex. We have not thought proper to adopt this nomenclature.

The bad cocoons which can be reeled, are generally reeled together; those which cannot are considered as waste silk, and are carded and spun, as will be hereafter mentioned. 


\section{CHAPTER VI.}

\section{OF THE DIFFERENT QUALITIES OF RAW}

SIIK.

BEFore we enter fully upon this subject, some preliminary matters require to be explained.

Equality in the threads is the principal requisite in all raw silks that are spun at the reel, by which it is meant that all the threads shall consist in their whole length, as nearly as possible, of the same number of the fibres of the cocoon. But those fibres are so fine and so delicate, that it is impossible in drawing and joining them together to preserve exactly the same number of them, and a certain approximation is all that can be obtained. Therefore, when we speak of raw silk, we do not say that it is composed of exactly so many fibres, but of one to two or three, three to four, four to five, five to six, six to eight, eight to ten, ten to twelve, and as we increase in numbers, a greater latitude is given; thus we say twelve to fifteen, and fifteen to twenty cocoons, (one fibre from each cocoon,) and when we come to coarser silks, we say from twenty to thirty, thirty to forty, \&c. 
This equality is required, not only for the sake of the beauty of the thread, which, however, depends greatly upon it, but also, and principally, to prevent its constantly breaking in the operation of throwing, which occasions a great deal of waste, which, as we have shown, adds a great deal to the labor and expense, and more or less deteriorates the beauty and quality of the silk. The operation of throwing or twisting is all done by machinery, a blind instrument, the force of which cannot be increased or moderated at pleasure. Therefore the same force which will only give the required twist to the strong parts of a long thread will break the weak ones, and what it does not break it will either twist too much or too little, according to the strength or weakness of the thread. For all raw silks do not require to be twisted alike; organzine, for instance, requires a strong$\mathrm{er}$, and tram a weaker twist, and, in general, the strength of the twist is suited to the proportion required for the kind of stuff into which the material is to be manufactured, some requiring a very slight and others a very strong twist. Therefore the greatest attention is to be paid in reeling to the equality of the threads, and it is in throwing alone that it can be accurately known by the greater or lesser quantity of waste, how far that 
equality has been preserved. The value of raw silk in the market depends much on the reputation of a filature, in this respect.

Mr. Boucher, in his letter to the Chevalier de Pougens, which will be found in the appendix, seems to be of opinion that it is easier to reel silk from 12 to 15 and 15 to 20 cocoons, than organzine from 3 to 4 and from 4 to 5 . We have had a conversation with him, when lately at Paris, upon this subject. He meant only to say that the instruction of female reelers should not be hastened, by making them begin with reeling fine organzines, which might appear beautiful to the eye, and yet be defective; that though not so necessary, it was more dfficult to preserve equality out of a great than out of a small number of cocoons, and that by reeling out of a large number, and striving to maintain equality as much as possible, they would be better prepared to reel off the fine threads, which he admitted, was really the most difficult part of the art, and that which requires the greatest skill, care, and attention, as well on account of the fineness of the threads, and the greater degree of equality that was to be preserved, as because they required a stronger twist in throwing than any other kind of raw silk. We fully agree with him in this opinion.

We will now proceed to the immediate subject of this chapter. 
We have, in the preceding chapter, divided cocoons into four classes :

1st. Good or perfect cocoons of the first class.

2d. Good cocoons of the second class.

3d. Bad or imperfect cocoons which can be reeled.

4th. Bad cocoons which cannot be reeled.

It is of the silk of the two first of those classes of cocoons, that are made the elegant stuffs for which the United States pay so dearly, and for which it is the object of this treatise to enable them to pay, by supplying the nations from which they are purchased with the raw material, properly prepared and fit to be employed in their various manufactures. The profits to be derived from this source depend upon the degrec of perfection in the art of reeling which shall have been attained in this country.

The qualities of raw silk to be produced from these two classes of cocoons, are of three deno. minations.

The first is called organzine, the second tram, and the third singles. We place this the last, because it is not in so great demand as the two others.

The silks, however, receive these names only by a kind of courtesy, because they are not entitled to them until they have passed through the throwing mill, where organzine may be 26 
converted into tram and singles into organzine. But this only happens in cases of necessity. If the silk has been properly reeled, it is almost always applied to the use for which it is intended, and from which it receives its name. Manufacturers, therefore, give orders to filatures for so many bales of organzine, tram, or singles, meaning of the kind of raw silk intended to receive those forms in the operation of throwing.

Organzine is the perfection of raw silk. It is reeled only out of the first class of the best cocoons. It is that for which the highest prices are given in the European markets, but always in proportion to the intrinsic quality of the silk and the perfection of the reeling. Dr. Lardner* speaks of a quality of organzine, made in the contiguous departments of Ardeche and Gard, in France, which sells commonly for 50 francs, (10 dollars, and some times as high as 150 franes, (30 dollars) a pound. It is employed in Normandy, in the making of fine laces. We do not know that it is produced any where else. We have seen that the best Italian organzine sells at present in London for $\$ 750$ a pound.

Organzine is generally employed in weaving for the warp of the most elegant stuffs. It

* Page 94 London Edition. 
should not be reeled out of more than from 2 to 3,4 or 5 cocoons. Our correspondent at Manchester wrote to us that raw silk, reeled from six to eight cocoons, was in the greatest demand in England, but for what purpose it was to be used, he does not explain.

The silk called tram is generally employed for the waft or shoot of those stuffs of which the warp is made of organzine. It is generally made of the second class of the good cocoons. it may also be made out of the first class, and then it is of more value. When the rearing of the worms has been well attended to by the farmers, there may be a deficiency of the less perfect class of cocoons, which compels the reeler to employ the best class. We hope it will happen so often in this country.

Tram should not be reeled off (of goud cocoons) of more than twelve to fifteen fibres to a thread.

Singles are also made out of the second class of good cocoons, and they should not be reeled of more than twelve to fifteen fibres to a thread. They are called singles, because they are not, like other raw silks, doubled in throwing. They are much employed in the woof of certain mixed stuffs of cotton and silk, of which the warp is made of cotton. These stufis which in our day were called cotes palis, 
are intended for the markets of the West Indies and other tropical climates. They require but little twisting. We would recommend this kind of raw silk to the attention of this country. 'These light stuffs night be manufactured here, and would be a good article of exportation to the Southern climates, with which the United States have a frequent and consiant intercourse.

Singles, by doubling and a stronger twist, may be thrown into tram of a good quality.

The silk of bad cocoons, which.are susceptible of reeling, is all reeled of from 20 to 25, and sometimes to 30 fibres to a thread; but it is difficult to unite perfectly well this last number of fibres. This is the silk that is employed in Europe in making sewing silks, plushes, stockings, gloves, night caps and other kinds of hosiery. Floss silk is also made out of it, which is a kind of flat silk much used in embroidery. And lastly all coarse stuffs, vestings and other articles of a similar kind, are made out of this silk.

We have seen many articles of these various kinds, knit or woven out of American silk, and they have been exhibited as proofs of the advancement of the arts connected with silk in this country. But we regret to say that they were made out of the best silk of the best co- 
coons, and of course, at an immense loss, besides that they had not attained the degree of perfection of those imported from Europe. This made us observe in our essays that it was like making kettles and sauce pans of pure gold. This observation applies particularly to seving silk, of which we have said enough that we will not repeat here.

We shall lastly speak of those cocoons which are not susceptible of being reeled. 'These, whether they are discovered in reeling or in sorting, are put aside, with the waste silk, the floss, the ribs, the third layer of silk which falls with the chrysalis to the bottom of the boiler, from which, after the chrysalis has been extracted, are boiled in a mass in soap and water during from four to six hours, after which it is carded and spun at the spinning wheel. This silk is called filoselle or furt silk. It is employed in making stockings and other kinds of hosiery of the coarsest kind. Those articles are much worn in Europe, and being made of silk, though of the most inferior quality, are preferred by many to the articles of the same kind made of other materials.

We will now proceed to the mechanical operation of reeling. 


\section{CHAPTER VII.}

OF THE REEL AND OTHER APPARATUS.

The operation of reeling, as we have said before, consists of two distinct parts.

1st. The drawing of the silk from the cocoons and keeping the machine constantly supplied with regular thread.

$2 \mathrm{~d}$. The winding of the thread round something that performs the office of a spindle.

The first of these two operations is done exclusively by the reelers or spinners; they receive no aid from the machinery. In this consists the difficulty of the art.

The second part is performed by the machine, which is set in motion by the turning of a wheel.

The Piedmontese reel has, for more than one hundred years, been considered as the best for winding the silk drawn from the cocoons; various improvements of this machine have at different times been attempted, but after fair and repeated trials, they have been successively rejected as defective and producing particularly that blemish called glazing, which consists in the threads being so badly directed 
upon that part of the reel called the hasp, * that they are glued to each other, one of the most perplexing defects in the operation of reeling. No nation of Europe has contributed so much as the Piedmontese, to improve the reel for the winding of silk; but this machine did not at once reach the perfection which it has now attained. One of the principal points which first drew their attention was, that the fibres of which the threads were composed, reached the hasp without being embodied, that is to say, perfectly united to each other. In order to remedy this defect they first imagined to cause each thread of silk as it issued from the holes of the bow-bent iron, to pass over the circumference of two cylinders. This invention had two advantages: 1 st, to cause the different fibres of which the threads are formed, by being compressed upon the cylinder, and thus imbibing their liquid gum, to glue them together and

* This name is given in France, Italy and Germany to the great wheel of the reel on which the silk is wound, the outer bars of which are called the arms. There is no proper name in English for this part of the machine, we have, therefore, called it hasp, which is also a good English word, and, we think, appropriate.

"Hasp, a spindle to wind the silk thread or yarn upon." -Todd's Johnson's Dictionary.

"HAsp, a spindle to wind thread or silk on." -Web. ster. 
make the whole thread compact. $2 \mathrm{~d}$, that the compression upon the cylinders lessens the moisture of the threads, and causes them to be wound round the hasp in a dryer state.

This apparent improvement at first took favor, because it gave to the silk a finished appearance, which it never had before. But this method had also its disadvantages. The pressure upon the cylinders gave to the threads a flat form, whereas its perfection is to be as round as possible. Besides, it was found that this pressure was not strong enough to unite completely the fibres, they were not sufficiently dry, nor sufficiently smooth. Instead, therefore of those cylinders, they contrived to make the two threads cross by twisting fifteen or twenty times round each other, which is called crossing; this operation is performed between their passage from the holes of the bow-bent iron to the eyes of the rampins, or more properly, the guides fixed on the distributing rod. This innovation had great success; the silk acquired from that moment a very different and a far superior quality; the threads by being: thus crossed fifteen or twenty times over one another, became round and compact on leaving the point of crossing, by which the moisture was much better dried up; it arrested, likewise, the floating particles of silk which sometimes 
rose from the basin with the threads. Thus the silk was distributed upon the hasp in a purer and dryer condition, and of a perfect, round form.

We will not here describe a method invented by the celebrated Vaucanson of crossing the threads at two different places, to wit, below and above the distributing rod. This invention has been put aside, because the reelers cannot be brought to use it, for the threads thus crossed twice, are liable to be broken very often, and occasion great loss of time.

After the discovery of the crossing, the Piedmontese added several other improvements to their reels. They applied themselves more especially to the perfecting of the distributing rod and its guides, and to establishing between the roller and the pulley, which was then fixed at the end of the hasp, a proportion so exact, that the threads should continually take a separate position upon its arms; so that no longer lying upon each other, they might not be glued together. After the most careful examination, they concluded that they should never attain the end which they sought, as long as the roller should receive its movements from the hasp, by the means of a cord with. out end, because this cord, by the alterations to which it was liable, and which were con- 
stantly occurring, from the different degrees of moisture or dryness of the atmosphere, perpetually deranged the proportion established between the hasp and the roller. Besides, the cord without end, by its continual friction, gradually wears away the wood of the roller, and that of the pulley of the hasp, and by thus deepening the channels, causes an irregularity in the just proportion which ought to exist between them, and if the cord be too loose, it will slip upon the pulley of the hasp, or upon the roller, and in either case, the roller will not make its proportional number of rotations; for all these reasons they have abandoned the movement by the agency of a cord without end, * and have substituted for it four bevilled wheels, with an established number of teeth, in order that the advancing and retiring motion of the distribu-

* These improvements in the reel were made in Pied. mont, more than a century ago; for in 1724 when the celebrated ordinance was made, the reel had attained its present degree of perfection: yet such is the force of habit, that in some of the domestic filatures in France, the cord without end is still in use. We ourselves used it in our experimental flature at Philadelphia, because, at that time we could not get the Piedmont reel made without considerable trouble and expense, and we were also much pressed for time. 
ting rod with its guides, should be permanently regulated with each revolution of the hasp.

The Piedmontese have also increased the distance from the distributing rod and its guides to the hasp, and have at last fixed it at 3 feet 10 $\frac{3}{4}$ inches, (American measure, in order that the minute particles of water with which the threads of silk are charged, being longer exposed to the air might evaporate and the silk be wound round the hasp in a dryer state.

Every one will understand that the motion given by cog wheels is more perfect and more equal than that given by a cord without end, or a pulley; the first is easily calculated, divided, and distributed in the desired proportion. It is easy to determine and fix its graduation by the number of cogs which produce the movement, and thus be able to calculate those degrees to their smallest possible number; a perfection which cannot be attained by the other motion, the cord without end or the pulley not being susceptible of that geometrical correctness which is requisite to calculate and distinguish the progression of such a motion; this is as clear as it is incontestable.

A motion by $\operatorname{cog}$ wheels is much more multiplied and varied than a simple motion, every one will understand that ; such is the motion of the Piedmont reel, as we are going to see. 
In this particular case, in order that the threads in reeling do not lie parallel upon the arms of the hasp, but cross over one another, it requires a motion extremely multiplied and varied to produce that irregularity, and this is precisely performed by the combination in the four $\operatorname{cog}$ wheels of this reel, as we shall presently demonstrate.

'The bevil wheel, R, (see the plate) has 22 cogs; it gears with another bevil wheel, $\mathrm{S}$, not of $22 \mathrm{cogs}$, which would produce a simple motion only, but of 25. This irregularity in the number of $\operatorname{cogs}$, creates, necessarily, another one in the motion. The bevil wheel, F, of 35 cogs, which guides the distributing rod I, gears with another bevil wheel U, of $\$ 2 \operatorname{cogs}$, which is a second irregularity.

This double irregularity of movement which takes place between the distributing rod and the hasp, which moves all, forms also an integral motion, the effect of which is to imitate, in the unwinding of the cocoons, the same method employed by the worm in forming it; for it is a fact well known to naturalists, that the fibres of the cocoon are spun on it crosswise, and not in a straight line, (the foreign writers call this $z i g-z a g$,) which is exactly the same manner in which the motion of the Piedmont reel places the threads on the hasp, from which 
this great advantage results, that the threads which in reeling still retain much of their gum, by not lying close to each other on the hasp in their whole length, are not so liable to be glued together ; besides that the motion of the hasp has already dried, in some measure, the silk threads that are placed upon it; for such is the effect of the well calculated motion of the different parts of this reel, produced by the admirable arrangements of the $\operatorname{cog}$ wheels, that the hasp has had time to perform 875 revolutions, and the distributing rod 484 , before the threads return precisely to the same place. Consequently the operation of this ingenious machine is an imitation of nature, of which the industry of the worm, instructed by her is the prototype.

All the above improvements, suggested by long practice and experience, have brought the reel of Piedmont to the perfection which it has been so long generally acknowledged to possess, and all the attempts to improve it in Europe have been successively rejected.

We must be allowed to say here, that the greatest number of the supposed improvements that we have seen attempted in this country, were no others than those which had been thus unsuccessfully tried in Europe; we do not speak of those that are truly contemptible, to 
some of which we have alluded in this work; there are quacks and pretenders in every country; but it is well that it should be known that those alterations, for we will not call them im. provements, have not met with success any where, and that the Piedmont reel in its present form is everywhere considered as possessing a degree of perfection which it is difficult, if not impossible to surpass.

We will now proceed to give a minute description of this truly wonderful machine, which gives unquestionably to the silk a beauty and a quality before unknown, and which no other invention has yet and probably never will be able to attain.

\section{EXPLANATION OF THE PLATE,}

\section{Fiaure I.}

The length of the frame is 6 feet, 4 inches, the breadth 2 feet $1 \frac{3}{4}$ inches out and out, the height is 2 feet $4 \frac{3}{4}$ inches; from the front of the bearers, $\mathrm{AAA}$, to the centre of the front feet, $\mathrm{BB}$, there is one foot and one inch, from thence to the centre of the hind feet, BB, 3 feet $10 \frac{3}{4}$ inches. The bearers, AAA, are 3 inches in depth, by $1 \frac{3}{4}$ inch in width; the feet $\mathrm{BBBB}$, are of the same dimensions; 5 inches from the bottom they are framed together lengthways and crossways with braces, CCCC, of suitable strength, under- 
veath which, boards are fastened in order to form a room to serve to put the bad cocoons and other things. The top of the frame is fastened at each end, by two cross pieces, DD; the one in front to be at least 5 inches in width, which is called the shelf, and serves to the reeler as a place to deposit her bunch of twigs, and her cup of fresh water to cool her fingers, the other not more than 3 inches wide. Directly above the front feet, $\mathrm{BB}$, two posts, EE, are placed, that on the left side is 4 inches long, in which an iron pin, $G$, is fastened, on which revolves a bevil wheel, $F$, called the pinion, having 35 cogs or teeth; this wheel is fastened to a turned piece of wood about 3 inches in length, which fits loosely on the iron pin, G; upon this piece of wood an iron crank, $\mathrm{H}$, is fastened, which has 3 inches eccentricity, in which the distributing rod, I, fits loosely; on the other post, that is to say, the right one, there is a mortice, or opening, in which the extremity of the distributing rod plays. Four feet and 6 inches from the front of the bearers, AAA, two other posts, KK, are placed, about 5 inches high, in which the gudgeons, LL, fixed at each end of the nave, $M$, revolve; this nave is 4 inches square, the thorough length of the arms, NNNN, is 2 feet 10 inches, the thickness is $1 \frac{3}{4}$ by 1 inch $\frac{1}{4}$; the four pieces, 0000 , 
on the top of the arms on which the silk is wound, are 2 inches square, and 18 inches long, with a groove of three-eighths of an inch in the middle, and both sides rounded off; one pair of arms are permanently fixed in the nave, but the other pair are cut asunder and placed crossways upon the other, and fastened by two wedges, PP ; this mode is resorted to in order that by taking out the wedges, the arms which are provided with rule joints, may be drawn into the opening, by which means the silk on the arms will be slackened, so that it may be taken off. At one end of the nave, $\mathbf{M}$, is fixed a handle, Q, by which the whole is put in motion; on the other end, is a bevil wheel, $R$, with 22 cogs, called the little bell, which gears in another bevil wheel, $\mathrm{S}$, with 25 cogs, called the great bell, fastened upon a shaft, $T$, to which another bevil wheel, U, with 22 cogs, called the star, is also fixed, which drives that of $35 \mathrm{cogs}$, F, called the pinion, by which the distributing rod, $I$, is put in motion. In front upon the cross piece, D, called the shelf, a bow-bent iron, $\mathrm{V}$, is fixed, inclining at an angle of $45 \mathrm{de}$ grees, with holes drilled in it, through which the silk threads are led to and passed through the wire eyes of the guides, WW, fixed on the distributing rod, to the hasp.

Now, if motion be given to the horizontal 
bevil wheel, or pinion, by means of the other wheels and shaft, when the handle of the hasp is turned, it is evident that this will cause the distributing rod likewise to move to and fro, directing the threads which pass through its wire eyes alternately to the right and left, through a range equal to the diameter of the horizontal bevil wheel to which it is attached.

'The remaining apparatus for reeling is very simple, it consists only of a basin filled with water, in which the cocoons are put, and a furnace to heat that water to the proper degree. The foreign writers have taken great pains to prescribe the size and the form of the basins, and the material of which they are to be made. The ordinance of Piedmont requires, (Art. 15,) that the basins be thin and of an oval form, and even goes so far as to prescribe their depth. This attention to minutix, shows how much importance is attached in Europe to everything that may in the least contribute to the perfection of raw silk. We cannot say that we allow so much importance to this object; we rather think that a basin large enough to contain the necessary quantity of cocoons, and to beat and manage them with ease, and of a material not susceptible of rust, and not liable to soil the cocoons or make the water foul, will, be sufficient. In our experimental filature at Philadelphia; 27 * 
we had basins made of copper that might have contained two handsful of cocoons, and which answered perfectly well.

As to the furnaces, there is no particular form required; in our filature they were made of bricks and built on the ground in front of each reel, and so placed that the reeler might sit next to them with ease. Their height should be such that they should not rise above the level of the reel where the reeler sits, and their diameter proportionate to that of the basin. They should be provided with a flue or pipe to carry up the smoke, so that it should not offend the eyes of the reeler, and injure the color and brilliancy of the silk.

But since the invention of the admirable apparatus of Mr. Gensoul, furnaces are no longer used in the large filatures of Italy and France, but the water is supplied by steam to the basins through pipes, in the manner hereafter described. This apparatus has the advantage of distributing a sufficient degree of heat to an indeterminate number of basins, by means of a single furnace. It regularizes the labor of the reelers, it raises or lowers by small degrees and with rapidity the water of the basins to the required temperature; it preserves the purity of the water and increases the quantity of the silk reeled, because the women, no longer disturbed 
by the necessity of attending to their furnaces, perform their work without interruption, and with greater ease, and are not offended by the smoke arising from their fires.

The following description of this apparatus is taken from a report made by Dr. Terme to the society of agriculture of the department of the Rhone, of which Lyons is the capital. We are indebted for it to Mr. De Teste in his excellent work on the silk trade in France. An engraving of it will be found in the plate, figure 2 , which we have taken from the same work.

\section{DESCRIPTION-FIGURE II.}

The apparatus consists of a boiler provided with a steam gauge and a safety valve; the boiler is placed in a furnace heated by means of bituminous coal. The steam thereby generated passes into a main pipe which extends horizontally through the whole apartment at an elevation of about ten feet from the floor. From this main pipe, descending pipes branch out laterally, each of these is bifurcated, and supplies two basins with steam. These pipes are terminated by a tube, the extremity of which is perforated with small apertures that allow the steam to diffuse itself through the water in the basin. They are also provided with stop cocks near the end, by opening or closing which, the spin- 
ners are enabled to regulate the temperature; when steam is introduced into cold water, it produces a hissing sound that continues till the temperature is raised to $167^{\circ} \mathrm{F}$. From $178^{\circ}$ to $190^{\circ}$, which is the best point for spinning, the water experiences a very distinct tremulous motion, which, at $203^{\circ}$ passes into a lively ebullition; this is the proper temperature for beating the cocoons. These indications may serve in lieu of a thermometer, and are so used by the reelers, who being no longer diverted from their occupations yb the necessity of keeping up their respective fires, as they used to be, are enabled to attend more steadily to spinning. The steam that condenses in the basins furnishes a constant supply of distilled or perfectly pure water, which gives to the products obtained by this apparatus, a marked advantage over those resulting from the old process. A comparison made by the committee, of hanks of silk produced by the old and the new apparatus, showed the superiority of the latter. $\mathrm{By}$ comparing these hanks with some of the first quality of Chinese silk, the committee satisfied themselves of the great and decided preference to be given to that of French origin.

To this report Mr. De Teste adds: "This mode of working by steam, compared with the old process, shows a saving of three-fourths 
of the fuel, an increase in the quantity of the produce of labor, which may be estimated at one-tenth, and an improvement in the quality and brilliancy of the product. Moreover, by means of it, the white silk retains all its brilliancy." 


\section{CHAPTER VIII.}

OF REELING AND PREPARING THE RAW MATERIAL FOR EXPORTATION.

Having taken the preliminary precautions already spoken of, that is to say, picked the floss or loose silk from the cocoons, sorted them according to their different degree of fineness, and separated the bad, the double, and other imperfect ones, we will now proceed to explain the manual operation of reeling.

The kind of water in which the cocoons are to be reeled is of considerable importance; it should be either rain water, or that which is obtained from ponds or slow running streams, and which has been a long time exposed to the air; water which is called hard, such as that which is supplied by springs or wells, usually contains a large proportion of earthy salt, and will not sufliciently soften the gum of the cocoons; they will consequently wind off with difficulty, and their threads be liable to continual breaking.

This water being put in the basin of the furnace, $X$, let it be made just to simmer, but not come to boiling. The exact degrees of heat to 
which the water should be raised cannot be exactly defined, for some cocoons will require water heated from $168^{\circ}$ to $190^{\circ}$; and others from $190^{\circ}$ to $202^{\circ}$. From $178^{\circ}$ to $190^{\circ}$, which is the best point for spinning, the water experiences a very distinct tremulous motion, which at $203^{\circ}$ passes into a lively ebulition; this is the proper temperature for beating the cocoons.

For the hardest sort of cocoons the water re. quires to be more heated, but a less degree for the others. However, the heat cannot be ascertained until the reeler begins to spin, for then if the silk comes off in knobs, commonly called gouts, or lumps, it shows that the water is too hot; it must be immediately cooled and the fire abated. On the contrary, if the silk is with difficulty drawn from the cocoons, which is known by their often leaping out of the basin, the water is then evidently not hot enough sufciently to soften the gum, and the fire must be increased. It is evident, therefore, that all those things are to be regulated according to the experience of the reeler.

The reeler or spinner who attends to the management of the cocoons in the basin, must be provided with a brush of about six inches in length, made of the finest twigs, or tops of heath bound together, and made flat at the 
brush part-two or three handsful of cocoons are thrown into the basin, wherein they are submerged, with the flat part of the brush, during some minutes to soften their gum; the reeler must then press the cocoons with the flat part of the brush. This operation requires care and dexterity, for if the cocoons are struck roughly, their fibres, instead of coming off singly, will cling together in lumps, and there will be a great loss of silk and great trouble in reeling them off. By means of this operation the fibres of the cocoons thus gently pressed, will adhere to the brush, and will be drawn out by its means, when the reeler disengages them, and draws the filament towards her, until it comes off quite clean from floss or coarse silk, which is called the ribs, and the fine silk begins to appear. These preliminary steps are called the beating.

In the composition of the thread, the reeler takes a number of fibres, according to the fineness of the silk that may be required of her, and delivers the compound thread to the person who turns the hasp, who passes it through one of the holes of the bow-bent iron, $*$ fixed

* There are four holes, two on each side of the bow. bent iron, but one on each side is only used, and may be changed if the reeler should find that the hanks on the hasp, are too near or too far from each other. But four or even three hanks cannot be reeled at the same time. 
above the basin. Another thread is, in like manner, to be prepared, and passed through another hole at the other end of the bow-bent iron; the two threads thus composed are then twisted twenty or twenty-five times round each other, in order that the different fibres composing each thread may better unite together by these mutual crossings, and likewise that the whole may assume a cylindrical form, which is indispensable for the good quality of the silk.

After being thus passed through the bowbent iron, $\mathrm{V}$, and twisted, the threads are led separately through the wire eyes WW, of the distributing rod, $I$, and being thence conducted to the hasp, are made fast to its arms, NNNN, one on the right and one on the left, so as to form two hanks.

Now, both threads being thus fixed, the hasp is turned with a regular motion, and somewhat slowly, until it is ascertained that all the cocoons yield their fibres freely and easily, for it will happen that some of the fibres which were taken to compose the threads were false ones, because in taking off the floss, the ribs, and loose particles, you may have taken hold of one of these false fibres which will soon end in reeling, and must be replaced, to make up the number which is to compose the thread.

As soon as the cocoons yield their fibres free- 
ly, a much quicker motion is to be given to the hasp. This is, however, regulated by the reeler, according to certain indications; for if the cocoons should leap up often, and beat against the bow-bent iron, the motion of the hasp should be slackened, and the fire increased, and if the thread comes off in burrs, knobs, or lumps, it must be turned quicker, and the fire diminished. Of this, the reeler, who keeps her eye constantly upon the cocoons and the threads, must, as she sees occasion, apprize the person who turns the hasp; that it may receive its proper motion, which ought to be as quick as possible, without endangering the breaking of the thread, or hurrying the reeler, so that she cannot add fresh cocoons when required.

To keep the requisite degree of evenness in the thread requires attention, skill, and practice. The reeler must not wait until the fibre of a cocoon is entirely exhausted before she puts on another, because as we have already explained, as the cocoons approach to their end the fibres become much finer. It is indispensable to attend to this circumstance, as well as to the breaking of the threads, in order that the requisite degree of evenness, without which the silk cannot be profitable, may be preserved throughout the threads. This gradually decreasing thickness of the 
fibres is in such a proportion, that where half wound cocoons, after hreaking, are again added, two such are considered equal to one that has not been used. Thus the union of three new and two half wound cocoons is equal to four new cocoons, therefore if the reeler does not take care to preserve the equality of her threads, by adding fresh fibres to strengthen them, they will break at the crossings. As we have already said, she must not wait until one or more cocoons be finished before she joins others to her threads; for by such delay she would not only expose her threads to break, but she would make besides a very unequal and bad quality of silk; and as far as it may depend upon her, she must prevent the threads from breaking; and when this accident is too frequent, it may generally be imputed to her carelessness or to her want of skill.

When the reeler neglects to purge completely the cocoons before the reeling, knobs of loose silk rise with the fibres and stop either at the holes of the bow-bent iron, $\mathrm{V}$, or at the crossings, and cause the rupture of the threads.

If she do not conduct skilfully the beating, so that the cocoons be on all sides well steeped, the silk will not wind off ; the cocoons will rise with the threads and make them break.

The cocnons must not be reeled off to the last, 
because when they approach their termination, the husk or pellicle of the worm, is carried forward with the threads and makes the silk foul. When the silk is nearly wound off, those small pellicles remain, and the cocoon being then too light to continue in the basin, will rise to the bow-bent iron, and if not immediately removed would, by stopping the passage, oc. casion the breaking of the threads.

It will not unfrequently happen in reeling, that the two threads in passing from the bowbent iron to the distributing rod, will become entangled with each other, and pass on together to the hasp, and this, from the rapidity of the motion, may continue for some time without being observed. We call that marriages. When this is perceived, the reeler must immediately order the turner to stop, and then the hasp is to be turned on the opposite side, so as to unwind the silk to the place where the marriage began. When it does not extend to any great length, the united threads are separated, and one of them is broken off, and the reeling continues; but if it should extend to too great a length, then a knot is to be made at both ends of the united threads, and the reeling goes on; when the silk comes afterwards to be wound off on bobbins from the hanks, which is the busi- 
ness of the throwster, $*$ those united threads are broken, and considered as waste; therefore it is of great importance that a reeler should pay the greatest attention to prevent these accidents which are the cause of considerable loss.

The supplying of fresh fibres requires readiness and dexterity which can only be acquired by practice; and there are no reelers sufficiently expert to give the necessary attention to more than two threads at the same time; it is proper, therefore, never to reel more than two threads at once.

In order to be always ready to add fresh fibres, to replace those which break or come to an end, the reeler must from time to time throw an additional supply of cocoons in to the basin for this object. Even this little matter requires to be done with judgment, for if any of the cocoons should remain too long in the basin, their gum would be dissolved, and the silk would come from them unequally; the same thing would happen if the reeler was under the necessity of leaving her work for any length of time; in that case the cocoons should be all taken out of the basin, until her return.

Raw silk may be wound of any size, from two cocoons, to one liundred, but it is very difficult

* See page 326. 
to unite more than thirty fibres in one compact thread. The art consists in reeling an even thread; for as the fibre of each cocoon is not of one uniform tenuity throughout, the skill of the reeler is required so to form her threads, that the same thickness may be continuously preserved throughout the hanks. This perfect equality is so difficult of attainment, that the degree of thickness in the silk is never exactly defined; and, with the exception of a thread of two cocoons, which is so called, silks are not distinguished as being composed of three, four, or five fibres, but as we have already observed, are said to be of three to four, four to five, or five to six cocoons. Coarser silks are not even so particularly defined, but are called from twelve to fifteen, from fifteen to twenty cocoons, and so on.

The reeler must always, during the operation of reeling, have at her side a bowl of cold water, into which she may dip her fingers; for without that precaution, she would be unable to bear the heat of the water in the basin. With that bowl of cold water she may also, from time to time, as occasion requires, abate the heat of the water in the basin.

It is not only of consequence to the facility of reeling, but also to the quality of the silk produced, that the water should be of a proper tem- 
perature, and as clean as can be; and it is for these reasons that $\mathrm{Mr}$. Gensoul's apparatus is so useful in large filatures, and should be generally adopted in establishments of that kind. As to those who wish to reel their cocoons on a smaller scale, it is necessary that they should be told, that the water in the basin must be changed twice a day at least, when good cocoons are reeled, and four times a day when dupions or other bad cocoons are wound. The chrysalides and husks contained in the cocoons very speedily make the water foul, and, for that reason, these frequent changes are needed; for if the water employed be not very clear, it will be in vain to look for the production of fine, brilliant silk.

Two hanks are wound upon the hasp at the same time; when these are completed, they are set in the shade to dry, without being removed from the hasp, which, for this purpose, can be readily disengaged from the frame. These two hanks will occupy the morning's labor. In the afternoon a fresh hasp is employed, and two other hanks are wound, which are set apart until the morning, that they may be dry before they are removed from the hasp.

Although this operation needs no instruction, yet we shall take occasion here to mention some things which will be found useful.

One cannot consider attentively the manner 
in which the silk is reeled from the cocoons, without observing that the fine fibres of which the thread is composed, are liable to suffer very different degrees of stretching, as they are winding from the cocoons. If the cocoons are not well sorted, this different degree of extension will be greater, and, even when they are well sorted, they are still subject to different stretching, because some always remain a little longer in the hot water than others, and therefore yield their silk more easily, and also, because the finest fibres of some cocoons wind off with the strongest of others.

The fibres being thus stretched unequally, will occasion (when the hank is taken from the hasp too suddenly,) those fibres which are most stretched, to contract more than others, by which their union will be in some measure destroyed, and the thread composed of them, rendered less compact and firm, the single fibres appearing in several places disjoined from one another.

To prevent this, the reeled silk, should not be suddenly taken off the hasp, but remain on it until the unequal extension which it suffered in winding, is, by the stretching which it undergoes, brought as much as possible to an equality, and until the thread by being well dried, has all the fibres of which it is com. 
posed, firmly united. This is only effected by having two hasps for each reel, so that when one is full, the reeler may proceed to wind upon the other.

When the skeins are quite dry; and while they remain on the hasp, they must be well cleared of all knots, or gouts, loose threads, (if any there be,) and other impurities; this must be done with the hand alone, any other mode is expressly prohibited by the ordinance of Piedmont, as will be seen in the tenth chapter; after this, the silk is taken off by taking out the wedges, and folding the arms. Before that, each skein is then to be tied round in two places with some of the refuse silk, it is afterwards doubled into a hank, and is considered ready for use or sale.

We repeat here that evenness of thread, and the absence of knobs or gouts, which, among manufacturers give to silk the name of being foul, are the leading points which determine its value in the market.

As to the floss, coarse and loose silk which was on the outside of the cocoons, it is carded and spun on the wheel; as is also the third layer of the cocoons, being first softened in water and soap. We need not give any information to the farmers as to the manner of performing this ope. ration; they are sufficiently familiar with it. 
The further management of silk, with the various manners in which it is thrown, and prepared to be woven, do not come within the perview of this treatise, but will, nevertheless, be briefly explained in the next chapter.

Sorting the silks for exportation is a subject which will require great attention and care. In the year 1774, when a filature was established at Philadelphia, under the patronage of the American Philosoplical Society, at the suggestion of the illustrious Franklin, Dr. John Morgan, an active and zealous member of that institution, carried on a correspondence on the subject of silk, with Messrs. Hare and Skinner, then respectable silk merchants in London. 'Two of those gentlemen's letters were published in 1785, (alas, too late, for the filature had then disappeared, in the transactions of the Society.* In one of those letters, dated July 27, 1774, Messis. Hare and Skinner write as follows: "The large quantity of raw silk that continually arrives from China every year, being mostly of a round or large size, will a good deal interfere with the sale of yours, provided you make it of the same; therefore, we by all means recommend your reeling yours of the fineness of five or six cocoons, no coarser at any rate, if

* Vol. ii, p. 347. 
avoidable. And we further beg leave to recommend your giving orders to your workmen to be extremely careful in assorting the silk, observing that all that is put into one parcel, be exactly, if possible, of the same fineness, for if it is not, it will very much prejudice its sale; a neglect in this particular, is complained of in all the silk that has hitherto been received from America. If the silk, which was very good in itself, that we received from Georgia, had been properly assoried, we certainly should have sold it $1 s .6 d$, or $2 s$. , per pound, better than we did. If you reel your silk fine, the China silk will rather promote its sale than otherwise, as it is necessary to have fine silk to work up with that of China."

This letter is suited to the time in which it was written. If large filatures should be established in this country, the managers will do well to follow the instructions of their correspondents in England and France as to the qualities of silk that are to be sent, as they vary with the different stufis which are made in the manufactories, and these depend upon the fashions of the day.

But the reeling and sending proper assortments of raw silk, will require the greatest attention. If a bale of that article should contain silks of various dcscriptions, without attention 
to what may be wanted, it will give infinite trouble to sort them afterwards, and will greatly lessen the price of the article. The reelers, therefore, when they leave a filature to set up for themselves, should be instructed as to the qualities of silk that will be wanted of them, otherwise they will work at a venture, and those who purchase their silk will be at a loss what to do with it. This, alone, is sufficient to show the advantage of large filatures, which will serve as guides to the domestic reelers, facilitate their work, and increase their profits. 


\section{CHAPTER IX。}

OF THE PROGRESS OF RAW SILK UNTIL IT COMES TO THE HANDS OF THE MANU.

\section{FACTURER.}

We do not mean in this chapter to instruct the reader in the various arts to the operation of which our precious material is subjected after it comes out of the hands of the reeler, when, and not before, it becomes entitled to the denomination of raw silk. We have already said that those different operations have nothing to do with agriculture, and that they belong to different mechanical trades or professions. Our object is simply to show the different changes which silk undergoes after reeling, until it comes to be manufactured into an immense variety of forms, which require different preparations, as well in the reeling as in the throwing of the delicate material. We also wish to show the impossibility of performing any one of these operations at the same time with reeling and by the same machinery, and that the so much spoken of machine, which is said to reel and twist silk at the same time, is either the produc. tion of imposture or of ingenious ignorance. 
From the filature, the silk, after being pro. perly reeled, and the hanks dried, cleaned and made up in the manner above directed, passes into the hands of the throwster, to be converted into what is called organzined, or thrown silk. The principal operation here is twisting, which is done by means of the throwing mill or machine, which is very complicated and fills a large room. But before the silk is put to the mill, it is to receive several preparations, by means of separate subordinate machinery. Those preparations are:

1st. Winding on bobbins. The raw silk, which the throwster receives in hanks, is now wound upon bobbins for the facility of twisting. This is done by means of what is called a winding machine, consisting of several slight reels all of the same form called swifts. They are placed at some distance from each other, so that several hanks may be wound at the same time. Dr. Lardner has given a drawing of one of those swifts, in his Treatise on the Silk Manufacture, page 199, Lond. Edit.

?nd. Cleaning or purging. Dr. Lardner does not speak of this second operation, for what reason we know not; but we have seen it con. stantly performed in France, in the throwing establishments, and it seems to be absolutely necessary, because silk, particularly that of the 
domestic filatures, seldom comes to the throwster perfectly free from knobs or gouts, and other impurities. These are removed by means of an instrument called the cleaner, of which we have not seen a description any where.

3rd. Doubling. This operation is that of joining two or more threads of the same silk together, according to the nature of the different fabrics in which it is to be employed. Some silks receive, before this operation, a slight degree of twisting at the mill, and a stronger one afterwards. All silks do not require the same degree of twisting, nor are they all to be twisted in the same manner, but some in a right hand, some in a left hand direction, and others in both directions. See Lardner, pp. 203, 204, where a drawing of the doubling machine is given.

The silk is now ready for its final twist. This operation needs not be described. Those who are curious of knowing it, will find a description of it with drawings, in Lardner's interesting work.

From the throwster, the silk goes to the dyer, who first boils it in soap and water, to dissolve the gum that still adheres to it; afterwards he dyes it of various colours, and hands it over to the manufacturer, who weaves it into different stuffs. Sewing silk, however, is perfect, after it is 
thrown and dyed. It is then fit for use and requires nothing to be added to it; so that the manufacturer of sewing silk is properly the throwster. It is easy now to perceive that even sewing silk cannot be made at the same time, nor by the same machinery which is employed to prepare ran silk. Much less can organzine, tram or singles, be so twisted, that are destined for the making of different stuffs, most of which require a different mode and degree of twisting. 


\section{CHAPTER X.}

\section{PIEDMONTESE ORDINANCE,}

CONCERNING FILATURES AND SILK REELS, Of the 8th of April, 1724.

Article I. Every one who shall wish to establish a filature of silk, whatever may be his quality, shall every year before he puts his filature into operation, present himself at the office of the Secretary of the Consulate, if he (the owner) lives in the city of 'Turin or its districts, and at the office of the ordinary judge, if he lives in another town or village, and there shall make his declaration, and bind himself to observe and cause to be observed, the following regulations, under the penalty of losing the reeled silks or their value for want of such declaration and engagement.

The Secretary of the Consulate shall keep a particular register of the declarations for the District of "Turin.

The ordinary judges shall transmit the declarations made before them to the said Secretary, within fifteen days after they shall have been made, otherwise they shall pay the costs and charges of the commissioners whom that 
magistrate shall be obliged to send upon the spot to receive them.

Of these the Secretary shall keep a separate record.

In case those declarations shall be made by the servants and not by the owners of filatures, the latter shall be civilly responsible for such declarations.

ArT. II. Every filature of more than three reels, shall while in operation, be superintended by an able and skillful person who shall watch over the observation of the following regulations. The owner shall make him known to the Secretary or to the ordinary judge, under the penalty of 25 golden crowns, (about $\$ 545$.)

ART. III. In order to reel silk, the good cocoons must first be separated from the double, spotted and imperfect ones; the floss must be cleanly taken off, and the various qualities must be reeled separately from each other, putting into the basins a sufficient number of cocoons, according to the quality that is to be reeled; the reeler must besides be very attentive to her work, that the thread be drawn perfectly equal; the whole under the penalty of 25 crowns, to be paid by the owner or those of his servants who shall be present and consent to a similar mixture, and of ten livres Piedmont, (about 2 dollars) to be paid by the reelers for each contravention. 
ARt. IV. The silks must be spun of two threads only, so that there be on the hasp no more than two hanks; those threads must be crossed at least 15 times for fine and superfine silks, and for other silks a greater number of times, according to their respective qualities; for the thicker and coarser the silks are, the oftener must they be crossed, which must not be done while the hasp is turning. Whenever the threads shall meet, and roll up thus doubled into a single thread, on one of the two hanks, the hasp must be turned in the contrary sense until the place shall be found where the threads began to be joined, and the double thread, must be placed in the middle of the hasp between the two hanks, so as to form a little band which will serve afterwards to tie up the said hanks; it being forbidden to tie them up with any thing else under the above penalties, besides the loss of the silk.

Art. V. All the silks must come out well purged, very clean and very equal, according to their respective qualities.

Art. VI. The posts between which the hasp is fixed, and those which support the distributing rod ought to be placed at the distance of two Piedmontese feet, (equal to three feet $10 \frac{3}{4}$ inches American measure,) from each other, so that from the hasp to the guides there be a suf- 
ficient distance, that the crossed threads, as lias been said above, may come to the hasp drier and in better condition: all that under the penalty of 25 livres for every reel otherwise disposed, to be paid by the owner of the filature. Art. VII. The hasp shall be of no more than 48 nor less than 4.0 ounces (a Piedmontese measure) in circumference. Nevertheless all the hasps of the sarne filature, shall have an uniform circumference, under the above penalty of 25 livres, \&c.

ArT. VIII. The hanks shall not be taken from the hasp until they are perfectly dry; and therefore every reel shall be provided with two hasps and with four, if the reel is double, under the above penalty.

ArT. IX. The hanks of the first and the second quality shall not weigh more than 3 or 4 ounces each; nor those of the third and fourth quality more than from 6 to 8 ounces, under the above penalty.

Art. X. Each hank as soon as it is taken off the hasp, shall be folded with two folds only; and shall not be tied with threads, nor with waste nor floss silk; one end of the hank shall only be passed through the other, so that it may easily be known whether there is any fraul, and whether the reeling has been done according to this ordinance; all this under the above penalty. 
Art. XI. The water of the boilers must be changed three times in a day; at the same time the cocoons must be well cleared of their floss, in order that the silk may come out clean, equal, and without floss, as there must come out of each rub of cocoons (18 pounds and $12 \mathrm{oz}$. French) at least about one pound of floss, regard being had to the quality of the cocoons, and this under the penalty of 10 livres for each. contravention, to be paid by the reelers.

ART. XII. (This article is merely fiscal.)

Art. XIII. The reelers shall be paid by the day, and not by the job. In case of contravention the owner shall lose all the silk reeled, and the reeler shall pay a fine of 20 livres, besides the loss of her salary.

Art. XIV. Every furnace shall be provided with a pipe or flue, of a sufficient height that the smoke may not fall on the hasp, under the penalty of 25 livres to be paid by the owners.

Art. XV. The boilers or basins must be oval and thin, and a quarter of a ras deep, (one foot American measure) each provided with a wooden cover. The reels shall have two sets of cog-wheels. The large horizontal wheel, or pinion, which moves the distributing rod, shall have $35 \mathrm{cogs}$ or teeth; the one underneath called the star shall have 22 ; the one next to the nave of the hasp called the 
great bell, shall have 25 teeth; and the one fixed to the centre of the nave, called the little bell, shall have 22. That machinery must always be kept in good condition, it is expressly prohibited to make use of reels with a cord without end ; the whole under the above penalty of 25 livres.

Art. XVI. Every reel employed in spinning silk of the first and second qualities, shall have a skillful person to turn the hasp, to whom it is prohibited to do it with the feet, under the penalty of 5 livres.

Art. XVII. It shall not be lawful for the reelers or any one else to cleanse the silk either on or out of the hasp, with needles, bodkins, or any such instruments, under the penalty of 10 livres.

ArT. XVIII. Under the same penalty it is forbidden to smooth the hanks on the hasp or elsewhere with any kind of liquid, even with pure water, the silk must be cleansed with the hands alone, and with nothing else.

Art. XIX. All silks that shall be found to be defective, and not to have been reeled each according to its quality, in observance of the forms and the rules prescribed in and by the preceding articles, shall be seized without remission, and be moreover liable to the above penalties, after a simple summary recognition 
of the defects; those silks shall be publicly burnt, saving to the owner his recourse against whom it may concern. The throwsters respectively shall be obliged to denounce the defective silks that they shall receive, and to make known the person from whom they shall have received them, under the penalty of 25 golden crowns to be paid by the owners or directors of filatures who shall be found in contravention.

ArT. XX. As to ordinary silks, that is to say silks of the last quality, commonly called faggottry, after having separated, as is above prescribed, the good cocoons from the double or imperfect ones, the reeler shall in beating the cocoons, draw up the coarse filaments three times to the height of half a ras, (two feet, American measure, above the boiler, that the silk may remain well purged and cleaned, under the penalty of 30 sous for each pound of silk.

Art. XXI. In order to insure the due observance of these regulations, the consulate and the ordinary judges shall be obliged in the reeling season, respectively to visit or cause to be visited, by experienced persons, the places where silk shall be reeled, taking all necessary information to discover contraventions, so that they may proceed against the delinquents, and inflict upon them the above penalties. The or- 
dinary judges and those who shall be appointed by them to make such visits shall not demand or receive any fees for their labor, costs or charges, until the end of the law suit according to the taxations which shall be made by the consulate. 

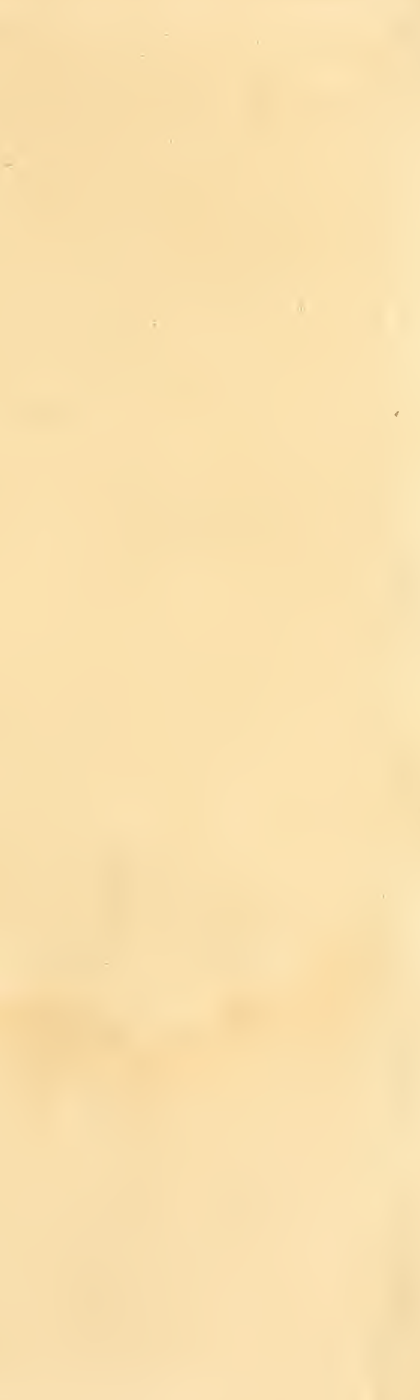
3

di

bJ

or

ch

to

co

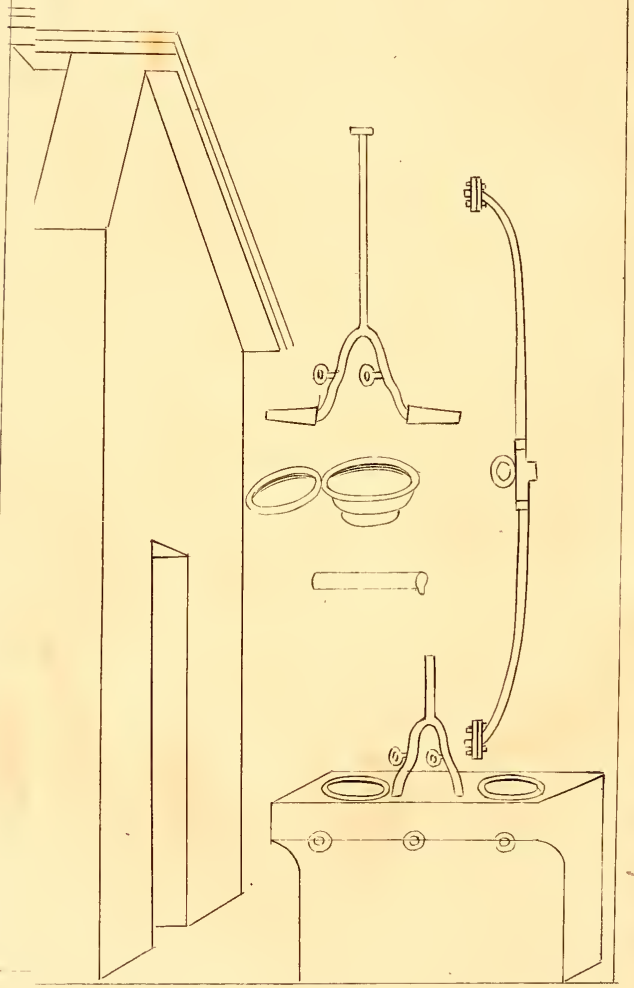

talles: 
A P P E N D IX. 
Plute

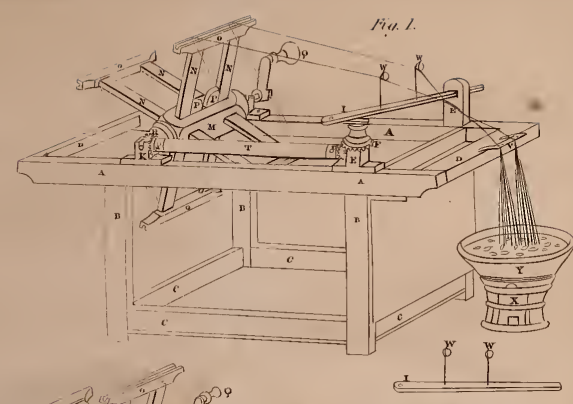

(15)

Silli. Ripel

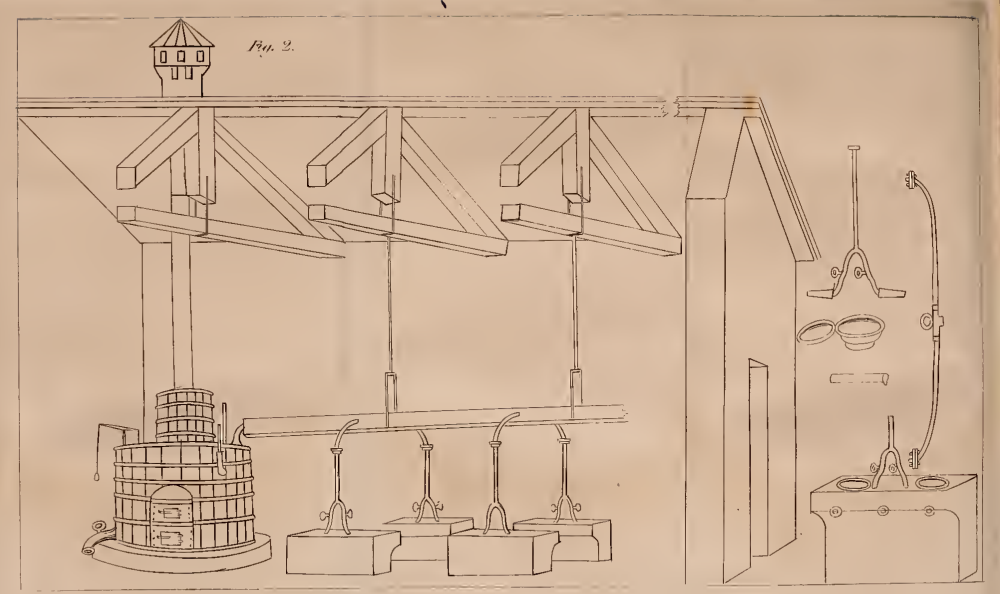

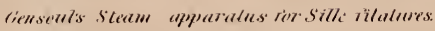





\section{A IPNDIX.}

\section{A.}

CORRESPONDENCE OF MR. BOUCHER OF PARIS, WITH MR. DU PONCEAU.

From Hazard's Register of Pennsylvania.

No. 1.

From the Chevalier de Pougens, to Peter S. Du Ponceau.

$$
\text { PARIS, 23d August, } 1830 .
$$

I enclose a letter from M. Boucher, the first of our Parisian merchants in the silk line, Rue Francaise, No. 2. I think I do you pleasure in communicating it to you. You may keep it.

No. 2.

From M. Boucher to the Chevalier de Pougens.

Paris, 20th August, 1830.

I am honored with your letter of the $12 \mathrm{th}$, instant. I must tell you with frankness that I do not at all believe in the success of the beautiful project of your honorable friend Mr. Peter S. Du Ponceau, of Philadelphia ; I consider it as the dream of an honest man. In support of my opinion, I will cite two gigantic undertakings which have failed within the last few years. I must refer you to the nine first lines of the fourth page of the French pamphlet you have addressed to me.* Yes, sir, practice is every thing

* This reference is to the French translation of the Report of the Committee on Agriculture, of the House of Representatives of the United States, of the 12 th of March, 1830.

The words referred to are in the English text as follows: "It is 
in this art, and theory very little. Your young man, M. D'Homergue, may have a great deal of knowledge of the silk trade, but he cannot have the knowledge and experience of old manufacturers; a thousand obstacles will arrest his progress, as happened to men of great experience, of whom I am going to speak to you.

In the southern part of Russia, on the confines of Persia, the Caucasian mountains produce a considerable quintity of cocoons, of which the inhabitants cannot make any use. Two Frenchmen, one of them Mr. Didelot, a great mechanician, and the other a man from our southern departments accustomed to the filature of silk, took with them an old director of filatures from St. Jean du Gard, and a few female reelers to found an establishment. They obtained from the emperor of Russia, large grants of land and buildings at Tifflis, large sums of money were advanced to them by the emperor; they began to reel silk, -we have seen some small samples of it that left nothing to be wished for; but they did nothing more, they could not supply their establishment with female reelers, the silk which those women reeled, apparently very fine, could not be thrown, and in the course of four years, all the capitals were sunk, the emperor abandoned them, one of them died, and the other returned to France, poor and destitute. The director of the filature and the female reelers could not return for want of money.

further demonstrated in those Essays, (Mr. D'Homergue's,) and in a memorial lately presented by the manufacturers of silk stuffs, of Lyons, in France, to the Minister of Commerce and Manufac. tures, that the art of filature can only be acquired by practical instruction, by some one intimately acquainted with, and accustomed to that process. That no human skill or ingenuity, unaid. ed by practical instruction, is capable of acquiring that art to any profitable extent." 
The second undertaking is still more extraordinary; it was made by a man of eminent merit in the sciences connected with the useful arts, and in the part of France where the greater quantity of silk is reeled (but by rote.) This undertaking has recently failed; out of a joint stock of $1,100,000$ francs, (220,000 dollars) the concerned will only have 15 per cent. of their capital, out of the proceeds of the sale of the real property. I send you herewith a printed copy of their articles of co-partnership,* which you may send to your friend Mr. Du Ponceau.

I do not mean to say that the project of establishing filatures of silk in the United States should be abandoned; but it should be pursued with prudence, if profit is intended to be made by it, and it must not be believed that in two or three years America can supply England and France with part of the silk they want.

The sixty young men to whom you will have in two years taught the theory of the art, will not be able to produce any thing, if they have not an overseer to attend to the quality of the silks which they shall cause to be reeled, and facilitate the disposal of it in the way of trade. They will meet with a thousand obstacles from the female reelers, and will for some years only produce raw silk unfit to be thrown, and yet those sills will appear very fine.

I would advise Mr. Du Ponceau to cause himself to be appointed director of a pattern filature, of which $\mathbf{M r}$. D'Homergue should be the overseer; that filature should be managed for account of the Government, and the object in view for the first year should be rather to instruct, and form female reelers, and male directors or overseers of filatures, than to make silk for sale. I would expend as little as possible in mechanical apparatus, and adjourn the employment of Gensoul's machinery; it is only good for

\section{- This document was never received.}


saving fuel in large establishments, and when old experienced females can be obtained, for raw silks of three and four cocoons fit for organzine, but which are entirely useless for the raw silks employed in making sewing silk and singles; the overseer may teach the art to grown women, and employ to turn the wheel girls from 12 to 15 years of age, who after a few months of exercise, will try to reel under the superintendence of the elder reelers; who will turn the wheel for them some hours in the day. It is thus that the thing is practised in the best filatures of France.

All those who have devoted themselves to the filature of silk, have imagined that the greatest merit was to be able to reel from three to four and from four to five cocoons, and to produce a thread regular to the eye ; but it is recognized by old reelers that it is easier to reel fine silk, than silk of 12 to 15 and 15 to twenty cocoons. The most essential quality of raw silk is to be easily wound or thrown and twisted, the more waste results from those operations, the more imperfect the silk is, and the more expensive the throwing; in my opinion the overseer, in order to hasten the instruction of the female reelers, should only permit them at first to reel threads of from 15 to 20 cocoons, the next day he should put another reeler at the basin, and employ the former in winding off the silk she had reeled the day before on bobbins, such as are used by the makers of sewing silk, so that it might be sold to them ready wound off.

That your filature may reach the desirable degree of perfection for raw silk of from 15 to 20 cocoons, it would be necessary that like that of Bengal, called Cossimbusar, they should suffer in throwing only a waste of one half per cent., or like the French silks of Alais and the Department of Gard, only one or two per cent; all other silks in the world from 15 to 20 cocoons, are worse reeled. "Those of Bursa, in Asiatic Turkey, lose from 6 to 8 per 
cent., those of Calabria, called Girelle from 8 to 12 ; those of Spain, called Tramas of Valencia, from 6 to 8; those of Syria from 15 to 25. Those of Saloniki and the Morea are still worse. Instead of attempting to reel silk of every quality, the United States should supply the trade with raw silks of from 15 to 20 cocoons, in bales of the weight of one hundred kilograms, (about 200 lbs.) Some years afterwards the same reelers might spin 10 to 15 , afterwards 8 to 10 and 6 to 8 for trams: in about ten years only, you might reel 5 to 6 and 4 to 5 for organzine, if the cocoons have nerve enough to bear twisting.

The skeins of Bengal silk are of the weight of two ounces; with a good distributing rod, they might without inconvenience be made of 4 to 5 ounces. The skein must be all of one single thread, and so that it may be fastened by crossing the first and the last end. There must be a good deal of twisting or crossing the threads in reeling, and the knobs or gouts must not be suffered to pass through. The selecting or separating of the cocoons is a most essential thing; all the double cocoons that we call dupions are to be put aside to be separately reeled, the stained are to be separated in like manner. The yellow and white cocoons must be separately reeled, to obtain a lively yellow and a pure white.

The silk will be handsomer, if the cocoons are first stirred in a basin of hot water of from $167^{\circ}$ to $203^{\circ}$ Fahr., then carried to the reeler's basin, the heat of which may be only $99^{\circ}$. You will thereby prevent the water being colored by the decoction of the chrysalis, and the silk from being tarnished.

The water in the reelers' basin must be changed at least four times a day. 'The more persons there are to oversee and watch the reelers, the more attention they will pay to their threads and to their basins. The broken threads must be tied up, and marriages (when two 
threads accidently join by passing through the same hole in the distributing rod) must be taken off. This is done in reeling silks of 3 to 4 cocoons, and still better in those of 15 to 20 .

They have been for some years in the kingdom of the Netherlands and in England, attending to the culture of the mulberry. Experiments have been made for raising silk worms, and it is pretended that they produce superb silks for their brilliancy. I am inclined to believe it, because in those damp countries, vegetation being quicker and the worm better fed, they must yield more abundant crops; but this speculation is only founded on experiments on a small scale. Mr. Christian asserts that a pound of silk may be made out of eight pounds of cocoons, while ten are commonly employed in silks of from 15 to 20 ; eleven in those from 10 to 15 ; twelve in those from 7 to 8 ; thirteen in those from 5 to 6 and 6 to 7 ; and fourteen in those from 3 to 4 and 4 to 5 cocoons; but $I$ think it is an error, produced by the dryness of the cocoons at the time of making the experiments. In fact $12 \mathrm{lbs}$. of cocoons, just out of the nursery, produce some months after only $8 \mathrm{lbs}$. because the humidity which is in the gum evaporates, and the chrysalis itself loses of its weight by the baking of the cocoons, to prevent the moth from escaping.

In cold damp countries the feeding of the silk worms is very uncertain; because the leaves, wet by the rain, occasion diseases among the insects, and the white frost may in one single night destroy all the leaves, which is less likely to happen in Italy and the south of France. In those countries one half only of the eggs on hand is put to hatch, to divide the chance of an adverse temperature, and some weeks afterwards the other half is put out, if both succeed, the crop is more abundant; it rarely happens that the two crops fail. 
If you think that this letter contains any thing that may be useful to your honorable friend, Mr. Du Ponceau, you may transmit it to him. If it should be agreeable to him, I shall continue with him this correspondence on the subject of silk. I would recommend to him to begin on the small scale his experimental filature; he has nothing but the reels and the basins to purchase, to begin to reel.

I annex to this letter a sample of Spanish raw silk, reeled in the neighborhood of Valencia,* of the quality called Trams, of from 15 to 20 cocoons. This is only fit for thick stuffs or fine sewing silk. Raw silk of from 15 to 20 cocoons, is best fitted for sewing silk.

There is a great deal more to be said on the subject of filature; but I am obliged for a beginning to confine myself to what appears to me the most urgent,

Accept, Monsieur le Chevalier,

My respectful civility.

L. J. BOUCHER.

No. 3.

From M. Boucher, to Mr. Du Ponceau.

PARIS, June 3, 1831.

SIR :-I received on the 24th of last December, your interesting letter of the 18th November. A long sickness, of which I am now convalescent, has been the cause of my not having answered it sooner. I wish that the letter which I addressed on the 20th of August last, to your respectable friend the Chevalier de Pougens, may be useful to you. That friend will have sent to you some weeks ago, a pamphlet entitled "Du commerce des Soieries en France" (on the silk trade in France.) This work, written by a friend of mine, Mr. Leon De Teste, of Avignon, will interest you.

* This sample was never received. 
I have carefully examined your samples of white sewing silk.* It is what we call demie grenade, or rondelette. It is made of dupions, (double imperfect cocoons, $\dagger$ two threads of the raw silk are twisted together, then closely united by a second twist more or less strong, and thus sewing silk is made either white or to be colored. Our rondelettes; in the raw state, $\ddagger$ are worth here 15 franes per pound, avoirdupois, with a discount of $12 \frac{1}{2}$ per cent. To this you must add one franc 25 centimes for dyeing, 50 centimes for packing, the loss of weight after extracting the gum and dyeing, 25 per cent. and thus you have the price of dyed rondelette, 21 francs per pound. You must compare this price, deducting the freight and importation duties, with that your women get for it at home. It is a branch of industry yet in its infancy: this silk is very irregular, as well in the reeling as in the twisting. $\$$

Your project for introducing the filature of silk, is grand and deserving commendation; do not, however, go too fast in endeavoring to spin fine threads; you would do bad work. You will, indeed, have pupils well instructed, but the improvement of the female reelers by practice, will require years. As to the manufacturing of stuffs, it will require still longer time; and moreover it remains to be decided whether the high price of labour in your country, will not be an obstacle.

What you say of Mr. D'Homergue gives me great hopes

* 'This was a sample of sewing silk made after the manner of Connecticut. It was a most favorable specimen.

†This sample was made of the best silk of the best cocoons.

₹The writer speaks here of the French sewing silk.

§The writer gives his opinion as delicately as he can, and reluctantly at last comes to this conclusion which cannot be misunderstood. 
as to the reeling of raw silk; * he must know how much the reelers of Alais, Anduse and St. Jean du Gard have improved their filatures, while not far from thence at Salon, the art has declined, and it is the same at Tours. $\dagger$

Your experimental filature of ten reels, is becoming interesting. Your raw silk, spun of six cocoons, if the threads are regular, the ends tied in knots, marriages $\ddagger$ taken off, and the silk made up in small skeins, will suit to make trams of two threads, and even organzine fit for the manufacturing of Florence of Avignon-but it will have to stand the competition of our own silks, as well for the price as for the throwing. England at this moment is more in want of raw silks of from 3 to 4 and from 4 to 5 cocoons, than from 5 to 6. $\oint$ I can say nothing of Mexico.

I shall receive with great pleasure the sample of your raw silk which you intend to send me, and I shall tell you what may be done with it. I should prefer receiving silk of 15 to 20 cocoons, like the sample of Spanish silk called

* The writer seems at first to have considered Mr. D'Ho. mergue as a mere dealer in silks, not familiar with the various processes of their manufacture.

$t$ If the art of extracting silk from the cocoons were as easy as some pretend, there would not be so much difference between the raw silk made in different places even in the same country. There is no such difference in the spinning of cotton, flax, or hemp, the value of these depends solely on the quality of the material, not on the skill of the spinners.

\# Marriage is when two threads in reeling, through the inattention or unskilfulness of the spinner, pass through the same hole in the distributing rod, and thus get improperly joined.

\$ The texture of our silk is so very fine, that the English manufacturers to whom samples of it were sent, have desired that it slould be reeled from 7 to 8 cocoons, instead of 4 to 6 , which is only equal they say to 3 to 4 of Italiar silk. Notwithstanding this extreme fineness, our silk is found to excel in stregth and nerve. 
tramas de Valencia, which I have sent you.* I would advise you to try your silk by means of a proof reel, which you might get made of 400 turns of a wheel of an ell's (44 French inches) diameter. 400 ells by their weight in grains of mark weight, give the standard weight (titre) which is called deniers.t Say 15 grains or deniers, single thread, or 30 deniers double thread. By this means you will be in harmony will all the manufactories of $\mathrm{Eu}$ rope.

I am like yourself, very much astonished that you have boiled your raw silk, without throwing it, either from tram or organzine; it must have been twisted or crossed a great deal in the filature. The silks of Bursa in Asiatic Turkey of 20 to 30 excoons produce the same effect; but with six cocoons it is most extraordinary. Do not trust always to it, however ; a little too much boiling in the dyeing process, may render the silk unfit to be wound.

I do not conceive how you can have made a piece of stuff with one single thread of raw silk reeled from six

* This sample was never received.

t The word denier means literally a pennyweight, here it seems synonymous to grain. Denier is a technical term in the English as well as in the foreign silk manufactures.

The proof reel is employed to test the quality of the silks, a given length of thread well reeled gives an ascertained weight; if it should fall below or rise above the standard, it is consiclered ill reeled in proportion to the difference.

The Chamber of Commerce of Lyons in France, calsed a sample of American silk reeled by Mr. D'Homergue himself, to be thus tried by a sworn assayer. The report was, that "the raw silk obtaineıl in Philadelphia was of an extraordinary quality, well adapted to all the uses of fabrication. Its degree of fineness was 16 deniers. It was fine, nervous, good, regular, clean, of a fine colour; in short it united all the qualities that cuuld be wished for. Its price was estinated at 26 francs per pound ; at Nismes, afterwards it was estimated at $30 \mathrm{fr}$. 
rocoons, it must have been very light.* I understand better that you should have trammed your second flag of four threads; but your dyeing on the piece, particularly of two colors, must have made their silk look like an old piece of stuff dyed a second time, consequently without lustre and crispy. $\uparrow$

I have observed that in a temperate climate, silk is always more brilliant, nervous and mellow, with livelier colors than in rery hot countries; this comes from the nature of the mulberry leaf; but in those countries, there is the danger of white frosts and frequent rains, which are seldom experienced in Italy and India. This danger may perhaps cause the silk culture to be abandoned. Be so good as to give me some information respecting the temperature of the United States of America, particularly in the spring season.

I have been assured that the English have invented machines of iron and copper for throwing of silk, of much greater activity than that of our wooden throwsting mills.

I am well aware, sir, that the love of your country and not the thirst of gain, induces your efforts for introducing the filature of silk into the United States. I wish you complete success, and shall willingly contribute to it in every thing that will depend upon me. I shall with pleasure follow your progress. Write to me as often as you please, do not spare me, you will receive speedy answers.

I am, \&c.

\section{J. BOUCHER.}

* The writer speaks of the flag presented to the Legislature of Pennsylvania. The warp of the stuff only was of one single thread, which was doubled for the woof. The stuff was indeed extremely light : twelve feet by six feet and a half, weighed only nineteen ounces.

TThis mode of dyeing was alopted for the flag presented to Congress, because it was thought proper to make it all of one 


\section{APPENDIX}

B.

\section{THE HISTORY OF THE SILK BILI:}

In a Letter from Peter S. Du Ponceau, LLD. to David $\boldsymbol{B}$. Warden, Esq. late Consul of the United States, at Paris, Member of the American Philosophical Society, Corrcsponding Member of the Institute of France, \&.c. \&c.*

\section{Philadelphia, 29th July, $183 \%$.}

\section{Dear Sir,}

I have received your favor of the 24th of May last, in which you desire me to send you a copy of the report made by the Committee on Agrieulture to the House of Representatives of the United States, on the 12th of March, 1830 , which was accompanied by a letter from me to the chairman of that committee, dated the 23d of February preceding, in which, at his request, I suggested a plan for

piece, and there was no time to prepare a suitable loom to weave different colours. In consequence of this the stuff har not the lustre it would otherwise have had; but was not crispy. The warp of the flag presented to the Legislature of Pennsylvania was entirely white, which gave to the colors a changeable ap. pearance. This was wished to be avoided in the other.

* This letter has never been before published. It was written to Mr. Warden, to enable him to give some information to the Agricultural Society of Paris, of which he is a member, on the subject of the silk culture in this country. Mr. Du Ponceau had only a few copies of it printed for distribution among his friends. Ile sent a copy of it to Mr. Randolph, Chairman of the Committee on Agriculture of the House of Representatives of the United States, with his answer to some queries addressed to him by the Committee, who mentioned it in their report as an interesting document. 
In that situation, he applied to me for advice. I saw his letter of recommendation to the society. He was represented by their agent to be a person fully capable of fulfilling the objects which they had in view. He had been examined at Marseilles by men skilled in the art, and found sufficient. In his conversations with me, I found him intelligent, and consistent in the explanations which he gave of the various processes of reeling and throwing silk, of making. sewing silk, and of various manufactures of that article. I was struck with the idea that his acquisition might be important to our country, and I determined to take him by the hand.

The first thing to be done was to make his talents. known. He could not write our language, nor did he know the manner of addressing an American public. On my part, I knew very little of the silk business, to which I had never before turned my attention. I put myself under his tuition. He initiated me into the mysteries of la filature and le moulinage (reeling and throwing of silk); I read some books upon the subject, and, having him at my elbow, I wrote and published in his name, in the National Gazette, a series of essays entitled "American Silk," which became very popular, and were reprinted in almost all the newspapers in the United States. While writing those articles, I was struck with the importance as well as the difficulty of the art of reeling, without which the raw material not only cannot be manufactured, but cannot be exported, because the cocoons will rot in a sea voyage, and are too bulky and too light to bear a heavy freight. I observed that raw silk was an article in great demand in Europe, and that it might be made to us a source of considerable profit. I conceived the plan of dealing with silk as we had done with cotton, that is to say, to confine ourselves for ten or perhaps, twenty years to the exportation of the raw material, during which time we should become perfect in the mode of preparing it for foreign manufacture, and then 
and not before, we might begin to manufacture ourselves, The other arts, such as throwing, weaving, \&.c., we might learn from English manufacturers; but they could not teach us that of filature, because their country did not produce cocoons. None, therefore, but a Frenchman or an Italian could be to us a proper instructor in that art.

The essays were chiefly directed to developing and urging the adoption of this system. Some writers who had passed before, by means of some superficial knowledge, for great adepts in matters relating to silk, were impelled by their vanity to attack this plan in the newspapers, and pretended that there was no difficulty in reeling silk, and that we might begin at once to manufacture. I took no notice of those publications, and, therefore, they produced little effect. The general opinion was in favour of my system. This induced me to publish the essays with an explanatory preface, in the form of a pamphlet, of which $\mathbb{L}$ distributed the copies far and wide. I have sought in vain for one to send to you.

This was in the month of January, 1830. Congress were then in session. As a mark of respect, as is usual in such cases, I addressed to them a copy of the essays, not expecting any thing else but they would give it a place ine their library. They, however, were pleased to refer it to their Committee on Agriculture, then presided over by the venerable Ambrose Spencer, formerly chief justice of the state of New York, whose high character is undoubtedly known to you. I soon received a letter from that gentleman, in the name of the committee, approving of my plan, expressing the wish that Mr. D'Homergue should be employed in a national school of filature, and desiring me to confer with him upon the subject.

I accordingly conferred with Mr. D'Homergue, and, with his agreement, I proposed to the commitiee that he $31 *$ 
should be employed to instruct sixty young men in the art of reeling silk, and preparing it for exportation, so as to fit them to be afterwards directors of filatures. You know that the manual operation of reeling in large filatures is performed in Europe by females, under the direction of an overseer, as otherwise much loss would result from their unskilfulness, or from their neglect or inattention--the profits in this business depending on the quantity of well reeled silk which is daily produced. Twenty women, at least, were to be employed in the normal filature at or near Philadelphia, who should work at the reel before the young men, who also should be taught the manipulation practically, that they might direct it with effect, and instruct women in their turn. 'Their instruction was to last two years ; afterwards it was expected that they should be employed by the planters in the south, and companies in the north, and in the meanwhile the culture of silk, which was fast advancing in different states, might produce cocoons enough to set the business agoing. The young men were to be taught gratuitously, and maintain themselves at their own or their friend's expence. The women were to be paid for their labour.

Mr. D'Homergue was to receive from the government forty thousand dollars at the end of the two years, deducting therefrom the amount of the necessary expences, such as the hire or purchase of a lot of ground, the erection of buildings, the machinery, the purchase of cocoons, and other incidental expences, which were to be advanced by the government, but the materials at the end to remain the property of Mr. D'Homergue. I offered to be his security for the performance of his engagements, provided he were placed under my inspection and control. My personal services were to be gratuitous.

My object in proposing this plan was to introduce into the country a perfect and uniform method of reeling silk, 
and preparing it for exportation or manufacture, but chiefly with a view to the former; so that when American raw silk should have been imported into Europe, its name alone should have secured its reception and a favourable sale. Those who are acquainted with this branch of trade, know of what immense advantage this would have been to our country. As to the planting of mulberry trees, and raising of silk worms, I thought it might be left to experience, and the instruction to be derived from the numerous books published upon these subjects; but the preparation of good merchantable raw silk, and the providing of a market for cocoons, appeared to me what was to be attended to in the first place ; the rest, I thought, would follow of course.

I made the above proposal in a letter to the committee, who gave it their full approbation. I was desired to present it in the form of a bill, which I did, and the bill, such as I drew it up, with a trifling addition, was laid before Congress with my letter, and a most flattering report from the committee, warmly recommending the measure. It is the same report which you have desired me to send to you, and of which a copy cannot now be had.

The session, however, was too far advanced to take up the business, and it was of course postponed to the next meeting. But, to all appearance the report was most favorably received.

I had then a whole summer and the greater part of the autumn to dispose of before Congress should meet again, I determined to spare no trouble or expense to convince the nation of the advantages of a good method of reeling silk, and of the exportation of the raw material to Europe. I established in this city a filature of ten reels, and twenty women; went to Connecticut to purchase cocoons, and bought all that were brought to me from different parts of the Union. I put the whole under the direction of Mr. D'Homergue. Our filature was open to all who chose to 
visit it. Our women learned to reel with amazing facility, and though some of my friends in Europe wrote to me that it would take ten years before they were perfect in the art, I was convinced that they would compass it in a much shorter time.

I was not satisfied with that. I wished to display something that would strike the public eye, and maintain Congress in their apparently good disposition. I had two flags of the United States made of American silk, each twelve feet long and six feet wide; one to be presented to Congress, and the other to the Legislature of Pennsylvania. I had also some small articles made, such as cravats, handkerchiefs, \&c., which, together with one of the flags, were exposed to public view at the exhibition of the Franklin Institute in this city. I also sent a quantity of my raw silk to England and France, to be manufactured into stuffs, and distributed among our friends in this country.

Congress met in December, 1830. That was what was ealled the short session, and lasted only three months. Almost the whole of it was taken up by the trial of a judge in the Senate, at which the members of the House of Representatives were present. Nevertheless, a day was appointed near the end of the session for the discussion of the silk bill. In the mean time, I presented to the house the flag of the Union. I may say with truth, that it was received with enthusiasm. On a most flattering report of the Committee on Agriculture, Congress resolved, that the flag should be displayed in the hall of their sittings, over the full length picture of General Lafayette, where it still remains.* There is no doubt that if the bill could have been brought on that session, it would have passed almost unanimously.

* The flag presented to the House of Representatives of Pennsylvania, was received in the same flattering manner, and ordered to displayed in a conspicuous place in their hall. 
Unfortunately, on the day when it was to have been taken up, Mr. Spencer, the chairman of the Agricultural Committee, who was to have led the discussion on our side, and whose presence could not be dispensed with, was engaged as counsel in an important cause before the Supreme Court of the United States. The argument lasted several days, and the opportunity was lost. Congress adjourned without discussing the bill.

But so zealous were our friends on the subject, that $\mathrm{Mr}$. Spencer, passing through this city on his return to the state of New York, where he resides, brought me a paper signed by eighty-six members of Congress, expressing their sense of the importance of the silk bill, and their opinion that it would have passed at the last session, if it could then have been taken up. Mr. Spencer told me that it would have been signed by many more, but that it was done at the close of the session when a great number of the members had already departed for their homes, and others were preparing to depart. Those who signed that paper were afraid that Mr. D'Homergue might return to France--that I might get tired of the trouble and expense which this business occasioned me, and for which I never expected to receive compensation, and that it would consequently fail. I subjoin to this letter a copy of that paper. It is a literal copy. I have made no alteration in it, except interverting the order of the signatures, in order to show to what states the signers respectively belonged.

Thus I was encouraged to proceed. I went on with my filature, but not to the same extent as I had done the preceding year. I sent some of my raw silk to Mexico, where it found a good market. I sent some to England and France, not to be sold, but to be manufactured into gros de Naples, and returned to this country. The silk sent to England lost only three and five eights per cent. by what is called waste (déchet); that sent to Lyons lost 
more, but the stuff made of it was more delicately wrought. I kept up an active correspondence with those countries.

The silk I sent to England excited much attention there. Mr. Ewart, the member for Liverpool, mentioned it in Parliament. 'The newspapers in London, Liverpool, and Manchester, teemed with articles on the subject. The English were led to expect a new source of supply of raw silk for their manufactures, and we a new branch of American exports. A number of silk throwsters, weavers, dyers, and silk manufacturers of various kinds, came- to this country in the hope of finding employment; but were for the most part sadly disappointed, and many obliged to return home.

Such was the state of things when Congress met again in December, 1831. I went with Mr. D'Homergue to Washington, determined to remain there until the silk bill should be disposed of. During the session, which was very long, I received from England some of the pieces of gros de Nuples manufactured out of my silk, and distributed them among our friends in the capital, by whom they were much admired. Those from France did not arrive until after the adjournment. Every thing at first looked fair and prosperous; but the scene was suddenly ehanged.

'The tariff question was brought before Congress, and produced violent party animosities. The word manufacture, which had inadvertently been introduced into the title of the silk bill, became hateful to the opponents of the protecting system, and they fancied that if it should pass, it would lead to the laying of high duties on the importation of foreign silk goods. Yet the bill still had friends of both parties ; but as the contest grew warmer, so as almost to threaten a dissolution of the Union, their number gradually diminished, and at last it became decidedly a party 
question. The French minister, M. Serurier, no doubt from patriotic motives, opposed it with all his might--not, that I know of, by diplomatic communications, but by speaking warmly against it wherever he found an opportunity. He saw in it the source of great injury to France, while the manufacturers of that country rejoiced in the hope of being supplied with the raw material that they so much wanted. Whether that gentleman's opposition had any influence on the final result, it is not in my power to say. I rather believe it had not, though some members might have been afraid lest the passage of the bill might prevent or delay the payment of the indemnity, which afterwards brought the two countries on the verge of a war with each other.

The times were now umpropitious. My friends advised me to suffer the business to go over to another session; but I had already gone to more expense than I could continue to afford. I pressed Congress for a decision, and a day was accordingly appointed for that purpose.

On that day, the 22d of May, the bill was taken up and discussed in committee of the whole; the majority declared in its favour, and reported it to the House the next morning. A warm discussion took place, but in the interval the opponents of the measure had rallied, and their phalanx was not be resisted. I have been credibly informed that a paper was circulated in the House, stating that if the bill should pass, the President would be obliged to put his veto upon it, and that he hoped that his friends would not place him in that unpleasant situation. I am well satisfied that such a paper was circulated; but I believe that it was without the knowledge or participation of General Jackson. Extraordinary, and, indeed, some unfair means were employed. Several of the members who had signed the paper brought to me by Mr. Spencer, as I have before mentioned, voted against the bill, and some absented themselves when the question was taken. 
'The bill, however, was rejected only by a small minjority. Among the members who voted against it there were some, undoubtedly, who conscientiously believed the measure to be unconstitutional, and that Congress had no power to appropriate money to such a purpose; others were political economists, who thought that governments should not meddle with such matters, and whose favorite motto was, Let us alone. But on the whole it is plain to me that the bill was lost on party grounds, and not otherwise.

I was not present at the last day's discussion, but I was at that of the day preceding, in committee of the whole. The debates on both sides were published at the time in the newspapers. It gives me great pleasure to say, that full justice was done by all parties to my conduct and to my motives; and not a word was said that could be in the least personally painful to me, but, on the contrary, the greatest courtesy was shown even by my most inveterate opponents. The principal argument employed, was that the measure was unconstitutional. The most violent contented themselves with saying that it could only serve the purpose of setting up a foreigner (Mr. D'Homergue) in business, and that the public money could not be applied to such an object.

I had the pleasure to find that the members from Pennsylvania who were present, although almost all of the partty opposed to the bill, voted (all but two) in its favour, and so did the members from Connecticut, whose jealousy had been attempted to be excited. The south, generally, voted against it.

Thus finally defeated in my patriotic design, I took leave of my friends and returned home immediately. I made no complaints nor appeals to the people in the newspapers or otherwise, but turned my thoughts to other objects. I found that I had lost three years of my time, and about four thousand dollars of my money, in pursuing a phan- 
tom, which at last eluded my grasp. I awoke, as from a dream, and consoled myself with the proverb which says, that the shortest follies are the best.

Five years have now elapsed since that time, and you will be, perhaps, curious to know what has been done in that interval with respect to silk. The impulse that was given still continues; but a different course is pursuing from that which $\mathrm{I}$ had pointed out. Mulberry trees are planted in every direction; associations with large capitals are formed and forming, for promoting the culture and manufacture of silk ; the Legislatures of the states are enacting laws for the same object; but I do not see any where a filature or a market for cocoons, such as I had established at Philadelphia, where they were brought to me from all parts of the Union, and continued to be brought long after my filature ceased to be in operation. But they found no purchasers.

The people are beginning where, in my opinion, they should end, by establishing manufactories. 1 have said that a great number of silk throwsters and manufacturers had come to this country from England. Those men, wanting employment, have persuaded the people to manufacture the raw silk of China and Bengal, as they do in Great Britain, which they have begun to do, in a small way however, in the New England states; while elsewhere the farmers who raise silk worms, employ them in making sewing silk after the manner of Connecticut.* Nobody thinks of producing and preparing the raw material as an article for exportation. The opinion generally prevails that there is no kind of dificulty in the art of filature, and that any woman may reel silk perfectly without having received any instruction. I fear that much money will be sunk in these ill-concerted attempts.

*With the common spinning wheel. 
The American people are impatient and wish to do every thing at once. Go a-head is their motto, and they are not deterred by obstacles. Nothing to them appears impossible; they plunge headlong into a scheme, and pursue it until they break, or until it suceeeds. They now want to be silk manufacturers. I wanted them to be silk growers, and exporters of raw silk in the first instance. Time will show who was in the right.

My opinion is that large sums of money will be sunk in the course now pursuing, but that in the end America will be a great silk growing and silk manufacturing country. Americans must have their own way. It often reminds me of the answer of a French courtier to the unfortunate Maria Antoinette, who asked of him a small favour:--" $M a$ dame, si la chose est possible, elle est déjà faite; si elle est impossible, elle se ferce." It is the American character, drawn to the life. The word impossible is not in their dictionary.

I am, with great regard and esteem, Dear sir, your most obedient servant, PETER S. DU PONCEAU.

David B. Warden, Esq., Paris.

\section{DOCUMEN'T REFERRED TO IN THE ABOVE} LETTER.

We, the undersigned members of the twenty-first Congress, feeling a great anxiety that Mr. John D'Homergue should remain in the United States for the purpose of instructing the youth of this eountry in the art of reeling silk, and in the various processes for preparing the same for manufacture or exportation, agreeably to the provisions of

* Madame, if the thing be possible, it is already done; if impossible it shall be done. 
the bill for the promotion of the growth and manufacture of silk, now pending, and which could not be acted upon for the want of time, express our decided opinion that the bill would have met the approbation of the House of Representatives, and that we consider it a measure of great national importance, as regards the industry of the country, in the profitable employment of various grades of its citizens, and as creating a staple which will not only save to the nation many millions of dollars, now annually drawn from the country, but will introduce speedily a new branch of manufacture of incalculable value to the United States. March 1, 1831 .

MAINE.

George Evans, Samuel Butman.

VERMONT.

W. Cahoon,

Benjamin Swift, Horace Everett.

MASSACHUSETTS.

J. Varnum,

E. Everett,

George Grennell, Joseph G. Kendall,

John Reed,

Joseph Richardson,

John Bailey,

James L. Hodges,

B. W. Crowninshield,

John Davis,

H. W. D wight.

RHODE INLAND.

Tristam Burges,

Dutee J. Pearce.
CONNECTICUT.

R. J. Ingersell,

Noyes Barber,

J. W. Huntington,

W. W. Eils worth,

William L. Storrs.

NEW YORK.

A. Spencer,

John D. Dickinson,

H. B. Cowles,

T. Beckman,

B. Arnold,

Robert S. Rose,

Ch. G. De Witt,

Isaac Finch,

J. Hawkins,

Abm. Bokee,

John W. Taylor,

Phineas L. Tracy,

Henry R. Storrs,

Henry C. Martindale,

Timothy Childs. 
NEW JERSEY.

Lewis Condict.

PENNSYlVANia.

Wm. MeCreery,

Henry A. Muhlenberg,

John Gilmore,

W. Ramsey,

George G. Leiper,

Joseph Hemphill,

Samuel A. Smith,

T. Hartley Crawford,

Thomas H. Sill,

J. B. Sutherland,

D. H. Miller,

John B. Sterigere,

R. Coulter,

Joseph Fry, Jr.,

Harmar Denny,

Innis Green,

Joshua Evans.

DELAWARE.

Kensey Johns, Jr., MARYLAND.

G. C. Washington,

E. K. Wilson,

G. E. Mitchell,

B. I. Semmes.

VIRGINIA.

P. Doddridge,

John Taliaferro,

C. F. Mercer,

Robert Craig.
NORTH CAROLINA.

Lewis Williams,

IVilliam B. Shepard,

Edmund Deberry,

D. L. Barringer.

KENTUCKY .

R. P. Letcher,

Joel Yancey,

R. M. Johnson,

J. Kincaid.

TENNESSEE。

John Blair,

David Crockett.

OHIO.

Samuel F. Vinton,

E. Whittlesey,

Joseph Vance,

IV. Creighton, Ir.

Wm. W. Irvin,

Joseph H. Crane,

II. H. Leavitt.

LOUISIANA.

E. D. White,

H. H. Gurley.

INDIANA.

John Test,

R. Boon.

ALABAMA.

R. E. B. Baylor.

MISSOURI.

Sp. Pettis.

FLORIDA.

Jos. M. White. 


\section{APPENDIX.}

C.

\section{ADDITIONAL DOCUMENTS CONCERNING THE SIK BILL.}

\section{No. 1.}

Twenty-first Congress of the United States-First Session.-In the House of Representatives, January 27th, 1830.

The Speaker laid before the House a letter from Peter S. Du Ponceau, of Philadelphia, member of the American Philosophical Society, accompanied by a work on American Silk, and the best means of rendering it a source of individual and national wealth; which letters and the accompanying work, were referred to the Committee on Agriculture.

February, 10.

Mr. Spencer from the Committee on Agriculture, reported the following resolution, viz :

That the Committee on Agriculture be directed to inquire into the expediency of adopting measures to extend the cultivation of the White Mulberry tree in the United States; to promote the culture of Silk, by introducing the necessary machinery for reeling the same from cocoons, and acquiring and disseminating practical knowledge therein.

$$
\text { No. } 2 \text {. }
$$

Letter from the Hon. Ambrose Spencer, Chairman of the Committee on Agriculture to $M r$. Du Ponccau.

Washington, Feb. 11 th, 1830.

SIR,-The Committee on Agriculture reported yesterday a resolution, which was adopted by the House, and which you will see in the papers.

I have read with great interest your valuable preface to Mr. D'Homergue's Essays on American Silk, having read in the papers, the greater part of his essays before I came 
here; the Committee are unanimously of opinion that measures ought to be taken to secure to the nation the services of this gentleman, as an instructor to our people in the art of reeling silk from the cocoons. I am instructed by them to inquire of him through you, whether he would take charge of an establishment (say in this city) and instruet pupils in that art-what salary he would expecthow long he would continue to give instruction-what period of time would be necessary to teach a pupil the art of recling. In short we wish all the information necessary to be laid before Congress on the subject of gaining to the nation this invaluable art. My proposition to the Committee was, 1st. "I'o secure the services of Mr. D'llfomergne. 2nd. 'I'o get an appropriation for the erection or hiring suitable buildings in this city, to accommodate say 215 pupils, from the States and 'l'erritories in the same proportion as we are represented in the House of Representatives, the persons to be designated by the Executives of the States. 3rd. 'That these scholars shall be taught the art by Mr. D'Homergue. 4th. 'Flat others shall succeed them, if it shall be decmed necessary to the diffusion of this knowledge.

I have selected this plan with a view to avoid Constitutional objections.

You have thus an imperfect sketch of my plan, which I hope you will examine, and make such suggestions as oceur to you.

If we succeed in it, the nation will owe you the deepest obligation of gratitude, for I am enthusiastically not only your disciple on this subject, but an anxious to see it carried into immediate operation.

I beg you to write me freely and fully.

With high esteem and respect,

Your obedient servant,

AMBROSE SPENCER.

Peter S. Du Ponceau, Esq. 


\section{A I'P YNNDIX.}

No. 3.

\section{Pinladelphia, February 23, 18.30.}

SIR:-I am honored with your letter of the 11 th instant, which I have immediately communicated to Mr. D'Homergue. He desires me to convey to you, and the honorable committee, his respectful thanks for the favorable sentiments that you have expressed towards him; he wishes it also to be known and understogd, that, if suc:cessfully encouraged, it is his intention to settle himself permanently in this country which he has learned to appreciate.

The contents of your letter have been the subject of frequent conferencess between hirn and me. The various matters that it brought to our consideration, and the desire on my part to lay at once my whole view of the subject before the honorable comrnittee, and to do it with as much as possible of that clearnesss and precision which so extensive and complicated a subject requires, have been the cause of this answer having been so long delayed. The committee will, I hope, see, from what follows, that no unnecessary time has been employed.

The object of the committee appears to be to extend, throughout the United States, the knowledge of the art of preparing raw silk in all its varieties, for the domestic and foreign markets; and that it should be done in the shortest tirne, in the easiest manner, and with as little expense as possible.

With a view to this object, a plan has been proposed through you, and several questions asked of Mr. D'Homergue, with a view to obtaining his assistance towards carrying it into execution. The plan is the establishment of a normal school of filature in the District of Columbia, in which Mr. D'Homergue should be employed as an instructor at a fixed salary.

This plan is formed on a noble scale, and bears the stamp 
of the character of a great nation; it is such as would naturally occur to a man of liberal views and an enlarged mind, on considering the subject unconnected with its practical details. The knowledge of those details, however, which Mr. D'Homergue possesses, has convinced him that it would be liable to many inconveniences, which he hopes it will not be thought improper in him to suggest. In the first place, it would be very expensive, complicated in its mode of execution, and liable to various abuses; and, above all, it would be subject to the risk of ultimate failure, by bringing Mr. D'Homergue in contact, and, perhaps in collision, with persons not acquainted with his art, to which his ignorance of the language, manners, and usages of this country, might not a little contribute. This last reason alone would deter him from acceding to the committee's proposal.

For my part, sir, I must frankly acknowledge that, after giving the subject all the consideration in my power, I have myself come to the same conclusions as Mr. D'Homergue, and I am satisfied that it is not by employing him as a salaried instructor that the object of the committee is most likely to be attained. On the contrary, I am fully persuaded, that a degree of confidence placed in that gentleman, in the manner I shall presently mention, will produce to the nation the most satisfactory results.

After this candid statement, the committee will no doubt expect that I should explain to them Mr. D'Homergue's views, and my own, on this interesting subject. I shall do it with due diffidence; but, at the same time, with perfect freedom.

The committee, I am very sure, will do justice to $\mathrm{my}$ motives, and be satisfied that my feelings are congenial with their own. I shall lay before them a plan, the result of which is to be the dissemination of the art of reeling silk, in all its varieties, throughout the United States, in the 
short space of three years, and at the moderate expense of forty thousand dollars. So far I am convinced that the views of the committee will be fully met. They will also be pleased to find, that its execution must naturally be followed by the introduction of silk manufactures into the country. In other respects I must own that it differs from that which the committee had formed; but, in a matter of this importance, they will be willing to hear, though they should ultimately disapprove.

Before I begin to state the offers of Mr. D'Homergue, and the plan founded upon them, it is necessary that I should mention a few introductory facts.

The mechanical part of reeling silk in France and Italy is performed entirely by women. There are in those countries what are called great and small filatures. The former are large establishments, in which from 50 to 100 reels are at work; the women empluyed there are under the superintendence of a director, who is thoroughly acquainted with the mechanical as well as the mercantile part of the business. Those directors are brought up to the profession. The women employed in those large filatures are well paid, and generally remain there till the end of their days. The small, or, as we should call them, domestic filatures, are carried on in families, by the farmers' wives and daughters, who work from one to five reels, either with cocoons of their own raising, or those they purchase of their neighbors, by which they make a handsome income at the end of the year. Those women, in general, have preserved the art in their families from generation to generation; hence, you may have seen in the memorial of the merchants of Lyons, which I had the honor to send you, that these merchants complain of their imperfect routine; I believe their complaints are in part from jealousy, and the spirit of monopoly ; still I am not unwilling to believe, that the silk from domestic reels is not as per- 
fect as that which comes from the large flatures ; it sells, however, and the manufacturers know how to employ it. It must be added, that the French and Italian female reelers perform their work mechanically, and are, in other respects, of the grossest ignorance: few of them, if any, knowing even how to read and write. Our American women will prove themselves far superior in every respect, and their domestic filatures will produce perfect silk, because they will not have received their instruction through their great-grand-mothers, before the art was improved as it is now.

In this country there should be both great and small or domestic filatures; the former will be the depositories of all the improvements in the arts; the latter will contribute to the easc of families; and there is no danger of there being an excess of the article in the market. There must be filatures of all sizes and all dimensions, according to the means of those who will undertake them. Full and free competition can alone ensure success.

The first thing to be done, therefore, is to instruct, in the art of reeling silk, a sufficient number of young men, to disseminate it at once through the country. Those young men will, when instructed, set up filatures with a greater or lesser number of reels, according to their means ; in which they will be obliged to employ women, who will not, as in Italy and France, remain all their lives on wages, but, after a certain time, will return to their families, or get married and set up small filatures of their own, which they can do at a trifling expense. They will, probably, also plant a few mulberry trees on their farms, and the females of the family will raise silk worms, and produce cocoons to be reeled in the house. This is the course which the thing must infallibly take.

Thus much being premised, I now proceed to state the offers of Mr. D'Homergue. He offers to instruct sixty 
yourig men, to be designated by the Government of the United States. They should be healthy, intelligent, active, and dexterous youths, between the ages of eighteen and twenty-five, and having as much as possible a natural disposition to the mechanical arts. They will be instructed in the theory and practice of the art of reeling silk from the cocoons into the various forms or qualities of raw silk. They will be taught the theoretical and practical, the mercantile as well as the mechanical parts of the business, with the most modern improvements. They will, in short, at the end of the course of instruction, be enabled to take charge, as directors, of a filature, however extensive, to instruct women, and, in short, to do every thing in that branch which Mr. D'Homergue may do himself.

The course of instruction will require two years; that is to say, two seasons, from the 1st of July to the middle or end of November in each year. The young men will board themselves where they please in the neighborhood of the filature, which they will be expected regularly to attend during working hours, to receive their instruction. The remainder of the year they may dispose of themselves as they or their friends shall think proper.

It is intended by Mr. D'Honergue to erect his filature in the vicinity of Philadelphia, as the most convenient place, for reasons which shall hereafter be explained.

The course of instruction ean only begin on the 1st of July, 1831; the present year must be employed in preparations. Mr. D'Homergue will have, before the 1 st of July, to travel through several of the States, in order to secure cocoons, and at the same time he will instruct the farmer, as much as will be in his power, respecting the culture of the mulberry tree, and the raising of silk worms. Every year, in the winter or spring, during the three years, he will travel in the same manner, varying his course as much as possible; this will be necessary, on account 
of the scarcity of cocoons, which must be expected to last some time, and probably to the end of the contemplated three years. In the approaching Summer he will establish a filature near Philadelphia, with at least twenty women, so that the young men, at the next season, when their course will begin, may see it at work, and learn how such an establishment is to be directed. In the next year the filature will be enlarged for the immediate instruction of the sixty pupils, who it is expected will be sent all at the same time. Improved machinery will be imported from Europe, and every thing necessary will be done in the course of the three years, that the young men may be fully instructed, and enabled, when they return home, to set up filatures in their respective neighborhoods.

Speaking of machinery, I cannot avoid noticing the celebrated apparatus of M. Gensoul, so often mentioned in the memorial of the merchants of Lyons, and which it contemplates to introduce into all the filatures of France. As it is very expensive the memorial recommends to the French Government to distribute bounties to an amount not less than 600,000 francs $(\$ 120,500)$ by way of encouragement to those who will introduce it into their establishments.

This apparatus can only be employed in large filatures of at least thirty reels. Its advantages are the following:

1. It saves the time of reelers, who are not incommoded by fire and smoke, and are not obliged to stop and feed their fires, as the hot water is conveyed by pipes to their several basins, and the furnaces to each reel are unnecessary.

2. It preserves the purity of the water.

3. It raises or lowers the temperature of the water in the basins, to the required degrees of heat.

4. It saves three-fourths of the fuel, increases the quantity of the produce of labor, which may be estimated at 
ene-tenth, and improves the quality and brilliancy of the raw silk.*

Mr. D'Homergue intends, if his offers are accepted, to import this valuable apparatus, in the first place, as a model, which will soon be imitated, and perhaps improved by our ingenious workmen; and, also, to enable him to instruet the sixty young men in the knowledge of all its parts, and in the use of it. It is very complicated, and requires great care in packing and forwarding. It consists of a great number of pieces, which must be all marked and numbered, in order that it may be properly put up on its arrival. It requires therefore, the aid of a person profoundly skilled in the business. Mr. D'Homergue proposes to employ his father. IIe would expect that this apparatus, and what other machinery he should think it necessary to import, during the three years, should be free of duty.

By means of this plan, the United States would be in possession at the end of the contemplated period, of the means of offering to the markets of Europe and of this country, raw silk, of all its different qualities, in the highest perfection. The committee will have observed in the Lyons memorial, how much importance is attached to the perfection of the filature, and what complaints are made of the want of uniformity in the silk produced. It would be a most essential point, that the first raw silk which shall come out of the American filatures, should be as perfect as possible, and uniformly so, because in addition to

* This description is somewhat altered from the original. Mr. Du Ponceau had misunderstood the explanation given to him. A few similar errors found their way into the essays of 1829 , which are all to be ascribed to him, to whom the subject was at that time cntirely new. He has authorized the insertion of this note. 
the superior beauty of the material, it would at once give a name to American silk, which would establish its reputation on a solid basis, and make it every where sought for and purchased in a manner with the eyes shut. If the United States are determined to introduce this manufacture into this country, the committee will be sensible that it should not be done partially, and that as little as possible should be left to chance hereafter. A good method introduced every where, at once from the beginning, will with difficulty degenerate.

If this plan should be adopted and carried into execution, it will follow, that, at the end of the three years' instruction, three sorts of filatures may and will be immediately introduced into the different parts of the United States. 1. Large filatures, of thirty reels and upwards, moved by horse or water power, or by steam. 2. Middle sized filatures, of six reels and upwards, moved by hand in the ordinary way. 3. Small or domestic filatures, of five reels and below; these would employ the farmers' wives and daughters, and the slave population, through the country. The difference in the perfection of the silk between that which is reeled with Gensoul's apparatus, and that reeled in the ordinary way, is hardly perceptible, when the directors are good, and the reelers are attentive, and have been well instructed.

The reason why, in France, the silk reeled upon farms obtains a less price than that reeled in filatures, is, that the peasants' wives and daughters follow an antiquated routine, as has been mentionel. In this country the method would be uniform every where. Negligence and inattention alone could produce a difference in the value of the silk reeled in farm houses, and this negligence would carry with it is own punishment. The raw silk of China, much inferior to that of France, is still sure to find purchasers; 
and there is little doubt but that it must be, in time superseded by American silk.

When the art of reeling silk shall have been thus established through the country, it is to be expected that the article will abound in the land; and particularly if measures are taken, as will be presently mentioned, for promoting the planting and growth of mulberry trees. Mr. D'Homergue then will have a great many competitors in the business of filature, which if monopolized, would have enriched him ; consequently, it will be his interest, and it is his intention, if this plan succeeds, to turn his attention to manufactures, from which he expects he will be able to make a competent fortune, and at the same time to enrich the country. In this he knows he will also have competitors, (for it is said there is already in Boston a good English silk throwster, of the name of Edward Brown, who has brought with him the necessary machinery for the exercise of his profession,) but Mr. D'Homergue relies on his knowledge of the various arts connected with this business, and is not afraid of meeting competitors.

The course which the silk business will take when filatures of raw silk shall be established through the country, is expected to be as follows:

1. The fringe-makers, who are already numerous in this country, will be supplied with the article which they now import at a great expense, and in considerable quantities. An eminent fringe-maker of this city said, in my presence, that he imported raw silk, annually, to the amount of $\$ 20,000$.

2. The filoselle, or flurt silk, which will issue from the filatures, and needs not be thrown, but only carded and spun in the usual way, will be immediately employed by our industrious workmen in making stockings, caps, vestings, and other kinds of hosiery. 
3. The art of throwing silk, that is, of giving it the last preparation for the loom, is not so difficult of acquisition as the art of reeling from the cocoons, which is the foundation of all; silk throwsters will come over from England and France, and that branch of business will soon spread through the country.

4. The weaving of stuffs out of the three first qualities of raw silk, singles, tram, and organzine, will next follow. It cannot be expected that the beautiful gold and silver tissues and embroidered stuffs, for which the I,yons manufacturers are so eminently distinguished, will be introduced for a considerable tine. Velvets and satins also will be among the last that will be manufactured in America; but the Iyyons memorial, if I remember right, says that those rich stufis amount only to one-fourth in value of the silk manufactures of France, and that the other threefourths consist of those plain tissues which are yearly imported to such an immense amount into this country. It may be expected, therefore, (such is the opinion of $\mathrm{Mr}$. D'Homergue and my own) that the manufactures of plain tissues and those of mixed stufis of silk and wool, and cotton and silk, will rapidly extend themselves through the United States. Where is no extraordinary difficulty in the mode of weaving, and Mr. D'Homergue is fully convinced that our ingenious and industrious weavers will master that business in a short time.

But all depends on the reeling of the raw silk. It is the foundation of all; the sine qua non, without which, all undertakings comnected with silk must prove ruinous to those whe shall venture to embark in them. Fine raw silk alone, though it were never employed here, will, nevertheless, be a great source of riches as an article of commeree.

I have thus stated to you, Sir, as clearly as I have been able, the offer made by Mr. D'Homergue to teach that val- 
uable art, in such a manner as to disseminate it at once through every part of this country. I have shown, in as much detail as I have thought necessary to make it well understood, the manner in which this proposal is intended to be carried into execution ; and, lastly, I have endeavored to sketch a view of the effects it may reasonably be expected to produce. I have now to state the terms on which Mr. D'Homergue is willing to engage himself to its performance.

He requires the sum of forty thousand dollars, for which he will engage to instruct sixty young men in the art of reeling silk from the cocoons, as has been abovementioned. He will be, for that sum, at all the expense that will be required for carrying the plan into full and complete execution, and will never ask or apply for a single cent more, by way of advance, reimbursement, reward, compensation, or on any other account whatsoever. The forty thousand dollars are expected to do the business completely, and to leave something at the end for Mr. D'Homergue; what that may be cannot be calculated, but he hopes it will be sufficient to enable him to set up for himself in the other branches of the silk manufacture that he contemplates.

He has calculated that it will be necessary that the money should be advanced by the United States at the following periods: 1. Ten thousand dollars immediately. 2. Twenty thousand dollars on the first of March, 1831 . 3. Ten thousand dollars on the first of March, 1832. The reason for which the sum is doubled for the next year, is, that machinery will have to be imported from France, to a large amount in the course of that year. The reason why the money is thus asked for in advance, is, in order that the business may not, at any time, or on any account, be retarded for want of funds, and that all mar go on with the necessary rapidity.

Here, Sir, I am well convinced that it cannot be reason33 * 
ably asked, that this nation should trust with so large a sum a young stranger, who has not been above nine months in this country, and who ean give no pledge of his solvability in case of failure. This objection has struck me with the same foree with which it will strike the committee, and I have long been revolving in my mind the means, if any could be found, to get over it; at last I have come to the resolution to make the following proposal in my own name:

I have set my whole heart on the introduction of the manufacture of silk into this country; I know it is possible, and I know, also, that great honor awaits those who shall have been instrumental in it. I am ambitious of that honor; and, moreover, I have committed myself so far in recommending this course of proceeding, that I stand in a manner pledged for its success. I cannot better prove my firm conviction, than by the offer I am now going to make.

I am willing to interpose my personal responsibility between the nation and Mr. D'Honergue; I offer to act as a trustee, to receive the money, and to see to its application. Mr. D'Homergue is willing to place himself entirely under my direction; and in consequence, I shall be responsible for any misuse of the money to be thus placed in my hands; I shall be in fact the director, to whose control Mr. D'Homergue will be bound to submit. He shall receive no money but through my hands, and I shail see to its application. In short, I sliall act for the United States, in this matter, as I should do for myself, if I were in their place. For this service, I shall expeet no reward or compensation whatever. I am devoted to the object; I have leisure and health; it will be a pleasure to me to direct and watch over this great undertaking, and to enjoy its progress, and I shall consider it as a glorious employment of the latter years of a long life.

Now, Sir, that you and the honorable committee may 
not think that I am here guided by unreasonable enthusiasm, I think it right to state to you the grounds on which I am induced to make an offer, which, otherwise, might be considered by some as at least a rash act, but which is only the effect of a strong conviction of the probability, I had almost said, of the certainty of success ; indeed, saving unforseen accidents, I cannot conceive how the project can fail. My grounds are the following:

1. Of the talents of Mr. D'Homergue I have not the least doubt. His recommendations from Europe are explicit on that head. The silk that he has reeled in this country has been admired by respectable silk merchants from Lyons, now in this country, who are competent judges. I enclose a small sample of raw silk, of the quality called organzine, which he has reeled in my presence, from cocoons sent to him by Thomas Sumter, Esq. of Statesburgh, South Carolina. They were most beautiful, and of an extraordinary size; this silk was shown in my presence to the fringe maker whom I have already mentioned, who mistook it for that fine silk with which they make Valenciennes lace, which he saw sold at Paris for $\$ 20$ a pound; but Mr. D'Homergue admits that it is not of that quality. This sample is very small, but there were but few cocoons reeled; and similar samples have been distributed among friends, and some sent abroad. The committee will, no doubt, be struck with the dazzling whiteness of the silk; such is seldom found out of this country.

2. As to the personal character and disposition of Mr. D'Homergue, I have had sufficient opportunity of knowing it, in a constant intercourse during nine months. He is a modest, ingenuous young man, ambitious of fame, and of perfectly correct principles. He places in me implicit confidence, and is willing to be, as he has hitherto been, entirely guided by my counsels. I can rely on his moral 
character; and I have not the least doubt that he will, under my direction, faithfully execute whatever he shall undertake to do.

I now shall state to the committee the reasons which convince me that he is the person the best calculated, and, perhaps, the only one, through whom the silk manufactures can be speedily and effectually introduced into this country.

His being the son of an eminent silk manufacturer has been attested to me, not only by himself, but by credible persons acquainted with his family. His knowledge is not confined to the reeling of silk, but extends to the various branches of the silk manufacture. Such persons are very rare, even in Europe; it was a fortunate accident that brought Mr. D'Homergue into this country. Silk reelers may be found, silk throwsters, also, and manufacturers skilled in particular branches; but none, or very few, and none at his age, possessed of so general a knowledge. His youth, too, is an immense advantage, as it will identify him with the country, and give him time to carry all his projects into execution. French reelers cannot be induced to leave their country; and if they could, as they are rery ignorant, and work mechanically, they would not be able to teach the art as Mr. 1'Homergue can. Directors of filatures are, in general, men of an advanced age, with families, well compensated for their labor; these could only with great difficulty be obtained; and it is very donbtful whether their talents for instructing would be equal to their pretensions, which, no doubt, would be very elevated. I consider Mr. D'Homergue as an important acquisition to this country.

Thus, Sir, I have ventured to lay before you and the honorable committee a plan for the firm establishment of the filature, and eventually, of the manufacture of silk in this country; which, after much reflection, and the most 
mature deliberation, has, and still appears to me to be, the cheapest, the easiest, and the most effectual, to produce this result completely, and in the shortest space of time possible. Permit me, before I conclude, to submit a few observations.

1. I believe it must be admitted, that, if the object can be attained for the sum of forty thousand dollars, without any further expense to the United States, it will be, considering its value, the cheapest purchase that ever was made. When we consider the sacrifices which the sovereigns of Europe have made for the same object, the noble rewards that they have given to individuals under similar circumstances; and when we consider, particularly, that, at the present moment, the government of France is called upon, by the merchants of Lyons, to expend the sum of $\$ 120,000$, in bounties to the owners of filatures, merely to induce them to purchase and use an expensive machinery, that the preparation of raw silk, long since known and practised in that country, may be uniformly carried to the highest degree of perfection, we may be able to judge of the importance and of the value in which is held in Europe that fundamental branch of the silk trade, on the perfection of which every thing else depends; and we are unavoidably led to the conclusion that forty thousand dollars is a trifling sum, indeed, for securing the introduction generally and uniformly through our country of so valuable an art.

As relates to Mr. D'Homergue, I do not think that less, in justice, can be offered to him, undertaking as he does to bear all the expenses. From the view he has taken of the subject, he does not expect, at the end of the three years, to have much more than the materiel of the establishment, which will, of course, remain to him, and perhaps some money. If it were otherwise, it appears to me a just principle, that he who makes a nation's fortune, 
should make his own. But Mr. D'Homergue does not expect to make his fortune by the execution of this plan, but only to be put in the way of making it by future exertions, which will also be highly beneficial to the country.

2. I regret very much that, as the committee contemplated, the execution of this plan cannot take place in the District of Columbia; but several weighty reasons are opposed to it. In the first place, there are not in that District the resources that are to be found in one of our large cities. 2ndly: In the employment of women, the differences of color might present great obstacles, which will not exist when several reeling establishments will be scattered through the States. 3rdly: I have already stated the difficulties which Mr. D'Homergue would find in his connexions with strangers, and which might eventually produce a failure in the project; and I am also convinced that, from my knowledge of him, his character and disposition, from the confidence that he places in me, and from my having become, in some degree, familiar with the subject of silk, and the various modes of employing it, he would more willingly place himself under my direction, than that of other persons with whom he might not so freely communicate. Under these circumstances, Philadelphia seems to be the place where the plan in question, if adopted, should be executed.

Nor does it seem very material where the sixty young Americans are taught, since their instruction is to take so short a time, and their acquired knowledge so soon to be diffused through the whole land. It would have been different if a permanent school were to have been established, as seems to have been contemplated by the committee. According to the proposed plan, the school will be only temporary ; and, in the course of two summers, the instruction of the young citizens will have been begun and completed. 
One more observation remains for me to make.

Whatever plan may be pursued for the introduction of the filature of raw silk into the United States, it will be indispensably necessary to take measures, at the same time, to increase the quantity of cocoons. It will be sufficient for that purpose, to encourage the planting of the white Italian mulberry tree, because, when it shall abound through the country, silk worms and cocoons will naturally follow. I would, therefore, take the liberty to suggest the expediency of granting a bounty, for a limited time, say five years, of — dollars for every three thousand such mulberry trees, of three years' growth; and if it should be wished to extend the benefit of it to small cultivators, then a proportionate sum for every thousand. The amount of the bounty should depend on the greater or lesser probability that there is of its producing the desired effect, so as to obtain the greatest possible quantity of mulberry trees at the least possible expense to the United States. I do not profess to be a judge in this matter. Mr. D'Homergue, on the supposition that the citizens would immediately and generally turn their attention to the planting of those trees, proposed fifty dollars for every three thousand; a gentleman from Indiana county, in this State, on whose judgment I place great reliance, on a contrary supposition, proposed one hundred dollars for the same quantity. But this is a matter on which the members of the Legislature are most competent to decide. If such a bounty were granted, it appears to me that no other legislative measure would be required.

I have endeavored, in this communication to be as clear, and, at the same time, as brief as possible; I am not sure, however, that I have succeeded. Should any further information be wanted from Mr. D'Homergue, or myself, it 
will be given with great pleasure, and every question readily answered.

I have the honor to be,

With the highest consideration and respect,

Sir, your most obedient

And very humble servant,

PETER S. DU PONCEAU.

Hon. Ambrose Spencer,

Chairman of the Committee of Agriculture of the House of Representatives of the United States.

No. 4.

Mr. Spencer's letter to Mr. Du Ponceau.

Washington, March $4 t h, 1830$.

DEAR Sir:-I ought to apologize to you for my seeming neglect in leaving your communication to the Committee on Agriculture of the 23d ultimo, so long unanswered, the reason of this delay is, that I wished to be able to state to you the views of the committee in relation to it. We have had the subject under consideration, and have agreed to your proposition, every member assenting thereto, except Mr. Wilson, who was out of town.

I can scarcely express to you the admiration your communication excited. To see a man of your age engaging so zealously in a plan promising such a rich harvest of reward to the whole country, presents a spectacle of devotion and patriotism, rarely seen in these days.

It shall be my first object to draw up a report, but I fear that I must yet trouble you further, in requesting you to draw a bill, and I will give you some suggestions in relation to it, to be followed or not, as you think best, desiring you to frame such a one as meets your own views.

1st. As to the $\$ 40,000$, ought it not to be payable to you, for the purposes specified in the bill? Mr. D'Homer- 
gue is a stranger, and there may be objections to placing in his hands that sum, my wish is to encounter as few objections in the details as possible.

$2 \mathrm{~d}$. Would it not be advisable to place the designation of the sixty pupils in the chief executive magistrate of each state? They are better able to make the designation, than any officer of the general government.

$3 d$. Ought not the pupils to be taken from the states in the proportion of their population or representation in the House of Representatives, giving every state one pupil?

4th. Should there not be some police established for the government of the pupils? On this subject, I have no very definite ideas, and I submit the whole to your consideration, asking only as speedy a reply as your convenience will admit.

Most Respectfully,

Your obedient servant,

\section{A. SPENCER.}

Peter S. Du Ponceau, Esq.

No. 5.

From the same to the same.

WASHington, March 13th, 1830.

Dear Sir,

Yesterday I made a report to the House, stating succinctly the principal points of view which rendered it important to make provision by law, for securing the services of Mr. D'Homergue, as an instructor in the art of filature. I presented at the same time the bill you so kindly drew, and which entirely met the views of the committee; and your letter to me, detailing your opinion and reasons in relation to the school. We added one clause to the bill, in order to secure its passage and to do away objections. It confers authority on the President to suspend the payments of the money, if it shall be made to appear to him. 
that from misconduct on the part of Mr. D'Homergue, the objects of the bill are likely to be frustrated.

The report, and bill and documents, were committed to the Committee of the Whole on the state of the union, and ordered to be printed; as soon as they are printed I will send you several copies.

I cannot but hope there will be wisdom enough in the Congress to earry this bill into a law. If this shall happen, you will have secured to yourself a title to the gratitude of your adopted country, and I shall have the satisfaction of being an humble auxiliary in this good work.

$$
\begin{gathered}
\text { Most truly, } \\
\text { Yours, }
\end{gathered}
$$

A. SPENCER.

Peter S. Du Ponceau, Esq.

No. 6.

\section{IN THE HOUSE OF REPRESENTATIVES OF THE UNITED STATES.}

\section{March 12th, 1830.}

The Committee on Agriculture, who were instructed by a resolution to inquire into the expediency of adopting measures to extend the cultivation of the white mulberry tree in the United States; to promote the culture of silk, by introducing the necessary machinery for reeling the same from cocoons; and for acquiring and disseminating practical knowledge therein; make the following report in part:

That the committee have been greatly aided in their inquiries on these important subjects by essays published recently by Mr. John D'Homergue, a native of France, who came to the United States during the last summer. Mr. D'Homergue, being unacquainted with our language, has been assisted in the writing these essays by Peter S. Du Ponceau, Esq. of whom it is unnecessary to speak, he being extensively known as a gentleman of the most patri- 
otic views, possessing great scientific attainments, and an unblemished character. The committee, therefore, consider these essays, and the facts contained in them, as entitled to high confidence.

Among the facts developed are several of an important nature. It appears that American silk is superior in quality to that produced in any other country. In France and Italy, twelve pounds of cocoons are required to produce one pound of raw silk, whilst eight pounds of American cocoons will produce one pound of raw silk. That cocoons cannot be exported to a foreign market from several causes, their bulk, their liability to spoil by moulding on shipboard, and because they cannot be compressed without rendering them incapable of being afterwards reeled.

It is further demonstrated in these essays, and in a memorial lately presented by the manufacturers of silk stuffs of Lyons, in France, to the Minister of commerce and manufactures, that the art of filature can only be acquired by praetical instruetion, by some one intimately acquainted with, and aceustomed to, that process. That no human skill or ingenuity, unaided by practical instruction, is capable of acquiring that art, to any profitable extent. It is made manifest, that, although the culture of silk has been carried on for many years in some parts of the United States, and more particularly in Connecticut, it has been conducted very unprofitably, compared with what the results might have been, if the art of filature had been understood. The sewing silk made in Connecticut is from the best of silk, and is, after all, quite inferior to that of France and Italy; in these latter countries, sewing silk is manufactured from imperfect encoons, or from refuse silk. It appears, also, that, unless the silk is properly reeled from the cocoons, it is never afterwards susceptible of use in the finer fabrics.

It is a gratifying consideration to the committee, that the benefits from the culture of silk, and the acquisition 
of the art of reeling the same, will be common to every part of the United States. The climate of every State in the Union is adapted to the culture of silk; hatching the eggs of the silk worm may be accelerated or retarded to suit the putting forth the leaves of the mulberry. That tree is easily propagated from the seeds of the fruit, and is adapted to almost any soil.

The committee regard the general culture of silk as of vast national advantage in many points of view. If zealously undertaken and prosecuted, it will, in a few years, furnish an article of export of great value; and thus the millions paid by the people of the United States for silk stuffs will be compensated for by the sale of our raw silk. Theimportation of silk during the year which ended on the 30 th of September, 1828 , amounted to $\$ 8,463,563$, of which $\$ 1,274,461$ were exported; but, in the same year, the exportation of bread stuffs from this country amounted. only to $\$ 5,414,665$, leaving a balance against us of nearly two millions. The committee anticipate, that, at a period not remote, when we shall be in possession of the finest material produced in any country, the manufacture of silk stuffs will necessarily be introduced into the United States.

The culture of silk promises highly moral benfits, in the employment of poor women and children in a profitable business, whilst it will detract nothing from agricultural or manufacturing labor. The culture of silk will greatly benefit those States which have abundant slave labor, the value of whose principal productions, particularly in the article of cotton, has been depressed by over production. It is well ascertained, that, although France produces within herself much silk, she pays annually more than $\$ 20$,000,000 for imported silk. The committee have been unable to ascertain the amount of raw silk purchased from other countries in England, but they are satisfied the amount is large ; and that, in these countries alone, a ready 
market can be found for all the raw silk raised in the United States for many years to come.

The committee have, through their chairman, corresponded with Mr. Du Ponceau, and this report is accompanied with a communication from that most respectable man and useful citizen, exhibiting his matured views on this interesting subject. Mr. D'Homergue is now in Philadelphia, and unless sufficient inducements are offered to him to remain in this country, he will very soon leave it forever. He possesses, in an eminent degree, all the practical knowledge necessary as an instructor in the theory and practice of the art of reeling silk from cocoons, and manufacturing the same into the various forms and qualities of raw silk known in the silk trade, having from his infancy been instructed in all the various processes. It is believed to be almost impossible to procure from Europe another person so competent to impart a knowledge of these arts as Mr. D'Homergue is. 'The acquisition of his services and instruction is invaluable; and in the opinion of the committee, if he be suffered to leave the United States at this period, it would be a national misfortune. In the confident belief that Congress will, unhesitatingly, provide for the appropriation of a small and insignificant sum of money, in promoting a measure which cannot fail to realize to the nation such rich results, the committee have prepared a bill, which they beg leave to present.

No. 7.

Twenty-first Congress-Second Session. - In the House of Representatives.

December 13th, 1830.

The Speaker laid before the House the following communication :

SiR,

You will receive with this letter a silken flag bear$34 *$ 
ing the colors of the United States. This flag is made entirely of American silk, reeled from the cocoons, prepared and woven by John D'Homergue, Silk Manufacturer. The coloring has been done by the best artist he could procure in the eity of Philadelphia; he himself not professing to be a dyer.

The staff of this flag, with the eagle, measures about fifteen feet; the flag itself is twelve and a half long, and six feet wide. It is woven in one piece, without a seam.

I beg, sir, you will be so good as to present this flag, most respectfully in my name, to the honorable House over which you preside, as a sample of American industry, thus applied, for the first time, to the most valuable of American productions, and as a result of the efforts they have made during the last five years, for the promotion of the important branch of agriculture to which we owe the rich material of which this flag is composed.

I have the honor to be,

With the highest respect, Sir,

Your most obedient

And most humble servant,

PETER S. DU PONCEAU.

Hon. Andrew Stevenson,

Speaker of the House of Representatives.

Ordered, That the said communication be referred to the Committee on Agriculture.

December 21 st.

Mr. Spencer, of New York, from the Committee on Agriculture to which was referred, on the 13th inst., the letter of Peter S. Du Ponceau, announcing his presentation to the House of a silken flag, bearing the colors of the United States, made of American silk, reeled from cocoons and prepared and woven by John D'Homergue, made a report thereon; which was read, and the resolution therein submitted, viz: 
"Resolved, That the flag bearing the colours of the United States, presented to this House by Peter S. Du Poncean, of Philadelphia, made of American silk ; prepared and woven by John D'Homergue, Silk Manufacturer, in the city of Philadelphia, be accepted by this House, and that it be displayed, under the direction of the Speaker, in some conspicuous part of the hall of sittings of this House."

Having been read, Mr. Alexander moved to lay the resolution on the table; which motion being disagreed to, the said resolution was concurred in by the House.

On motion of Mr. Spencer, of New York,

$$
\text { February } 9 \text { th, } 1831 .
$$

Ordered, That the Committee of the Whole House to which is committed the bill (No. 345) for promoting the growth and manufacture of silk, be discharged from the consideration thereof, and that the said be committed to the Whole House on Monday next.

$$
\begin{gathered}
\text { No. 8. } \\
\text { Twenty-second Congress-First Session. } \\
\quad \text { December 14th, } 1831 .
\end{gathered}
$$

The Speaker laid before the House a letter from Peter S. Du Ponceau, of Philadelphia, dated December 9th, 1831 , upon the subject of the culture and manufacture of silk; which letter was referred to the Committee on Agriculture.

January 20, 1832.

Mr. Root, from the Committee of Agriculture, to which was referred a letter from Peter S. Du Ponceau of Philadelphia, on the culture and manufacture of silk, made a report thereon, accompanied by a bill (No. 294) for promoting the growth and manufacture of silk; which bill was read the first and second time, and committed to a Committee of the Whole House to-morrow. 
Thursday, February 2.

On motion of Mr. Root,

Ordered, That the Committee of Agriculture, to which is committed the bill (294) for promoting the growth and manufacture of silk, be discharged, and that the bill be committed to a Committee of the Whole House, and made the special order for 'Tuesday the 7th instant.

\section{February 16.}

The House proceeded to the consideration of the bill (No. 294) for the growth and manufacture of silk, (and of another bill) whereupon it was

Ordered, That the consideration of the said bills be postponed until Monday next.

\section{March 12.}

Mr. Root presented a memorial of the New York State Agricultural Society, praying that the bill now pending before this House (No. 294) for promoting the growth and manufacture of silk, may speedily be passed into a law, or that such other measures may be adopted, as, in the wisdom of Congress, may seem meet for the encouragement of the growth and manufacture of silk; which memorial was referred to the committee of the Whole House to which said bill is committed.

May 10.

The Speaker communicated to the House a letter from Peter Stephen Duponceau, stating sundry reasons why it is important to the nation that the bill, now pending before this House, "for promoting the growth and manufacture of silk," should be acted upon as soon as possible; and, above all, that it should not be suffered to lie over the present session; which communication was committed to the Committee of the Whole House to which the said bill is committed. The letter is as follows *:

* This letter is printed among the public documents, and marked, Document No. 232, House of Representatives. 


\section{Washington, 8th May, 1832.}

SiR-

The present session of Congress being far advanced, and business pressing on your honorable House from every side, I think it my duty to solicit again their attention to the bill "for promoting the growth and manufacture of silk," now pending before them, and to state some reasons why it is important to the nation that it should be acted upon as soon as possible, and, above all things, that it should not be suffered to go over the present session.

As there are many members of the present House who are unacquainted with the history of this bill, and who may not understand on what grounds I take the liberty to address them through you, I beg leave to give here a brief statement of it, which, while it serves as my apolngy, will, I believe, throw some additional light on the important subject to which your attention is most respectfully requested.

When, at the beginning of the first session of the last Congress, I had the honor to present to them a copy of the "Essays on American Silk," then lately published by Mr. D'Homergue and myself, I had nothing in view but to give them a mark of my profound respect, and, at the same time, through them, to extend the knowledge of the facts which the book contains. I was highly flattered by the honor which the House did to that little work, by referring it to their Committee on Agriculture; still, I had no idea that that reference would lead to the recommendation of a legislative measure.

The idea of deriving a national advantage from the exportation of raw silk was entirely new, at that time, in the United States. Until then, the culture of that rich production of our soil had been considered only with a view to domestic manufactures. This is so true, that, in the able 
report of the Committee on Agriculture, made to the House on the 2d of May, 1826, in the Manual that was prepared and published on their recommendation; and in the answers that were sent from all parts of the United States to the then Secretary of the Treasury, Mr. Rush, in consequence of his circular queries, (as far as those answers have come to my knowledge, the importance of the exportation of raw silk, as an article of commerce, is not any where suggested. Thus, our agriculture, as far as silk is concerned, was considered only as auxiliary to domestic manufactures; while the idea first thrown out and developed in the "Essays," contemplates solely the advantage of the agricultural interest of our country, which alone is to be benefited by the sale of our produce to foreign nations in the form of a raw material, manufactures may follow or not, as it may happen. In either case, our coun$\mathrm{t}_{\mathrm{ry}}$ is to be benefited by the sale of an article exclusively the fruit of agricultural industry. When we consider that the small country of Piedmont exported, in the year 1829, near five millions of pounds of her raw and thrown silks, we shall better understand the value of this suggestion. France does not permit the exportation of her silks, unless manufactured.

'These reflections could not but forcibly strike the minds of the enlightened committee to whom the work was referred. That committee did me the honor to address me through their chairman, and desired my interference to retain Mr. D'Homergue in this country. I was requested by them to ask him on what terms he would accept to be placed at the head of a national school of filature; in consequence of which, after having ascertained the lowest terms that could reasonably be offered to him, and obtained his assent, not without difficulty, as his pretensions were higher, and these I found justified afterwards by a letter from his father, in which, appealing to his experience of 
forty years, he told him that forty thousand dollars would hardly be sufficient to enable him to execute what he had undertaken; I submitted a plan, which the committee immediately adopted, and presented to the House in the form of the present bill, to which they subjoined my letter to them, and an able report, warmly recommending the measure, in which is found this remarkable expression, that it would be a national misfortune if Mr. D'Homergue were suffered to leave this country.

That report was made on the 12th of March, 1830. The session was then far advanced, and the House could do no more before their adjournment than order six thousand copies of the report, with my letter to the committee, and the "Essays on American Silk," to be printed. The Senate, to whom a copy of that work had been also presented, referred it to their Committee on Agriculture and manufactures, with whom I had some correspondence, which, however, produced no result, as the subject had been so fully taken up by the House of Representatives.

Under these circumstances, sir, I thought myself in honor, if not in duty bound to justify the confidence placed in me by retaining Mr. D'Homergue in this country, at least until another session of Congress. At the same time, I determined to prove to Congress, and to the nation, by the evidence of facts, the great importance of the contemplated measure, so that it might be popular by the time it should come again before the National Legislature. I went with Mr. D'Homergue to Connecticut, to see how the people there managed the silk culture, and their so much spoken of domestic manufacture of sewing silk. My object in going thither was also to purchase cocoons, of which however, I could obtain but a small quantity, as the people thought that they might employ them otherwise to more advantage. We, therefore, supplied ourselves, in that and the succeeding year, from other sources. On my return to Philadelphia, I erected an experimental filature under 
the direction of Mr. D'Homergue, in which American wo* men were instructed in the art of preparing raw silk for exportation. As there was not time before the then next session of Congress to obtain information from Europe of the results of these experiments, I prevailed on $\mathrm{Mr}$. D'Homergue, although without much of the requisite machinery, to manufacture, himself, in various forms, a part of the silk prepared at the filature. It was not until the end of the last session of Congress, that we obtained proofs from abroad, that our raw silk was esteemed in foreign countries, and might become a valuable article in our commerce with other nations. The details of these experiments and their results at home and abroad, have been made known to the House in former communications.

The last session was short, and a great part of it taken up by a State trial in the Senate, at which the members of the House attended. The House, however, showed its favorable disposition towards the bill, by fixing a day for its discussion; but, more pressing business intervening, that discussion did not take place, and Congress adjourned, of necessity, on the day appointed by the constitution.

At that time, sir, Mr. D'Homergue had received tempting offers from a foreign minister, then at Washington, and was hesitating whether or not he would accept them. The fact was known to many members of the late Congress, who considered it of the highest importance that Mr. D'Homergue should stay in this country, at least, to wait the result of the present session. To obtain that end, a paper was signed on the very day of the adjournment of the late Congress, by eighty-nine members of the House of Representatives, in which, without presuming to predict what might be done by a future Congress, they did not hesitate to give it as their decided opinion, that, if the bill had been considered at that session, "it would have met with the approbation of the House of Representatives." 
That paper was brought to Philadelphia, and delivered to me by the Hon. Ambrose Spencer.

Under such circumstances, what could I do? However inconvenient it might be to me to continue the efforts I had begun, that was not now an object for my consideration. A market had been established at Philadelphia for cocoons, and they were bringing in for sale from various parts of the country. To have discontinued that market at once, would have discouraged the farmers, and checked the impulse which Congress had been giving to the silk culture since the year 1826 ; and, what would have been worse, Mr. D'Homergue might have accepted some of the offers made him, and left this country. I therefore determined to persevere; the cocoons were purchased, the women hired, and the filature again set to work, not as a business, or with a view to any profit, but as a continuation of former experiments. Mr. D'Homergue was persuaded to remain in the United States, and to reject the offers made to him by the foreign minister to whom I have alluded, and by another, then in Philadelphia, who made overtures to him in my presence.

The period having arrived for the opening of the present session of Congress, I had the honor of addressing a letter to you, requesting that you would place the subject before the eyes of your honorable House. I had the satisfaction to see that my letter was promptly referred to the Committee on Agriculture. Encouraged by this favorable token, and determined that no effort should be wanting, on my part, to promote so important a measure, I resolved to accompany Mr. D'Homergue to this eity, that he and I might be on the spot to give to the members all the explanations that they might require. We attended together the Committee on Agriculture, who brought in the former bill with a report not less favourable to it than that of their predecessors. The order of the House, which 
soon after followed, to place that bill among the special orders of the day, convinced me of the high importance which they attach to the subject; and I should not think it necessary to trouble them or you with this letter, if the session were not so far advanced, and the prospect of the bill's being taken into consideration during its continuance diminishing every day. At any rate, the part $I$ have taken in this business, in consequence of the facts I have stated, and which I have continued to take as long as my means would permit, until prudence warns me to desist, if the House should postpone its decision to another session, will, I hope, be accepted as an apology for what otherwise might be considered as an officious and unwarranted intrusion.

I beg to be permitted to take this opportunity to say, that, from my own observation, and the information of others, and particularly of editors of newspapers, who, by exchanging their journals, have the best means of knowing the feelings of the people at large, the measure contemplated by this bill is highly popular among all classes of men, but particularly the agriculturists throughout the whole Union; and I am satisfied that, if passed into a law, it will give general satisfaction. I am further convinced of this, and that the postponement of the bill will cause great disappointment among the farmers who have turned their thoughts to the silk culture, by the letters which I receive from all parts of the United States, which generally end with the query, whether there will be a market for cocoons this year at Philadelphia? which question I am unable to answer. This inquiry has lately been made by the inhabitants of the silk district, in Connecticut, who two years ago, were unwilling to sell their cocoons, because they thought they could manufacture them to better advantage, but now appear to entertain a different opinion. From the southern States, similar inquiries are made; and, since I have been in this city, I have been informed 
that cocoons had been sent for sale to Philadelphia from North and South Carolina, but could find no purchaser, as it would be idle in me to purchase that produce, to throw away afterwards if the silk bill shall not pass. I ought to add that several State Legislatures have made laws to encourage the culture of the mulberry tree, and the breeding of silk worms, in contemplation of the passing of that bill. I fear that if a check be given to this strong impulse by the discontinuing of a market for cocoons, it will be difficult hereafter to revive it ; and, without the silk bill, I do not see how that market can be continued.

As I was going to conclude this letter, I received, from Philadelphia, extracts from a series of public documents lately presented to the American Philosophical Society, by Don J. M. Tornel, late Minister from Mexico to the United States. Among those documents, are official reports, in which it is stated that the want of the knowledge of the art of reeling is the only thing that prevents the culture of silk from flourishing in that Republic; and I ought to add, that it is known to me that overtures have been made to Mr. D'Homergue, on the part of that government, to induce him to enter into their service.

Thus, sir, three foreign governments have endeavored to obtain the aid of Mr. D'Homergue to introduce or perfect the art of reeling silk among them. 'This shows that it is not so easy, as some have imagined, to obtain persons thus qualified from other countries : and that this nation is possessed of an opportunity, which, if it should suffer to escape, it may long, very long, have cause to regret hereafter.

The only object of this letter is to endeavor to convince your honorable House of the high importance of this bill, in the confident expectation that it will be finally acted upon by Congress at the present session. In doing so, I conceive I am performing a duty, which, if I were to neg- 
lect, I would have cause to reproach myself for it forever after.

I have the honour to be, with the highest respect, Sir, your most obedient and very humble servant, PETER S. DU PONCEAU.

Hon. Andrew Stevenson,

Speaker of the House of Representatives

of the United States.

May 22.

The House Resolved itself into a Committee of the Whole House on the bill (No. 294) for promoting the growth and manufacture of silk; and, after some time spent therein, the Speaker resumed the chair, and Mr. Barringer reported the said bill to the House with amendments.

May 23.

The House proceeded to the consideration of the bill (No. 294) for promoting the growth and manufacture of silk : when

A motion was made by Mr. Polk, that the enacting words of the said bill be stricken out.

And, after debate thereon,

A motion was made by Mr. Wickliffe, that the said bill be recommitted-with instructions to inquire into the expediency of so amending the same as to establish at the public expense, a filature for reeling silk, and for the employment of suitable superintendents for the same, and of providing for the instruction of pupils in the art of reeling silk.

And, on the question, Shall the said bill be recommitted with instructions?

It was decided in the negative.

The question was then put on the motion of Mr. Polk to strike out the enacting words of said bill;

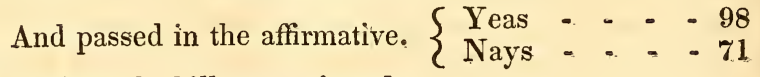
And so the bill was rejected. 


\section{APPENDIX.}

D.

Proceedings in the Legislature of Pennsylvan'a on the presentation of a flag made of American Silk, bearing the colors of the United States.

From the Harrisburg Reporter, Friday, January 6, 1831.

\section{AMERICAN SILK.}

A very beautiful flag manufactured by $M r$. D'Homergue of Philadelphia, from silk the growth and produce of this state, was a few days since presented to the Legislature by Peter S. Du Ponceau, Esq., accompanied by a letter from that gentleman. The letter was referred to a committee in the House of Representatives, consisting of Messrs. Ingersoll, Read, of Susquehamn, and Craft: and Mr. Ingersoll from that committee, on the 14th inst. made a very appropriate report, accompanied by a resolution that the flag be displayed in the House, under the direction of the Speaker. Agreeably to the resolution the flag has been very tastefully displayed over the Pennsylvania Arms in rear of and above the Speaker's chair, where it at once commands the attention and admiration of every one entering the hall of the House. We think it a fine specimen of the useful perseverance of its worthy donor, and a strong earnest of what may be expected from the culture and manufacture of silk in Pennsylvania hereafter. 'The following is the letter and report of the commitlee. The report was unanimously adopted.

\section{Philadelphia, Jan, 3, 1831.}

Sir,-I take the liberty of presenting through you to the honorable House over which you preside, and of offering to their acceptence, a flag, bearing the colors of the 
United States, and made entirely of American silk, by $\mathbf{M r}$. John D'Homergue, of and in the city of Philadelphia.

The texture of the flag is light and delicate; more so perhaps than it should have been, if my object were not by this specimen to show that stuffs of this description may be manufactured in this country, from our own native material. It is for similar stuffs that we pay annually to Europe a tribute of several millions of dollars, considerably exceeding the amount that we receive for all our bread stuffs. Hitherto the silk that this country produces, has been exclusively employed in making sewing silk, and a few stockings, gloves, and other like articles of domestic manufacture, in which the best material has been used, while elsewhere those articles are made of imperfect cocoons, and of waste and refuse silk. For more than twenty years, the inhabitants of a part of the state of Connecticut, have pursued this unprofitable system, and it is remarkable that the silk districts which ought to be the richest in that State, are in fact the poorest. There is no market there for their cocoons or silk balls, those who raise them are obliged to manufacture them themselves, or they. will perish on their hands. Nor can they find any eash price for the articles they make, so that they must use them in their families, or dispose of them by way of barter. This system is fast extending itself through the other states, and the only use that has hitherto been made of the cocoons in Pennsylvania, has been converting them into sewing silk, or coarse articles of domestic fabric.

It is not that manufacturers of fine silk are wanted in the United States, we have them in this city of every description, seeking employment which they cannot obtain for want of raw silk properly prepared. The art of making this preparation, which is called reeling, is not known among us, though it is generally but most erroneously supposed to be very simple, while on the contrary it reguires 
much instruction and long practice and experience; and those who know and would instruct us in it, cannot without the greatest difficulty be procured from other countries. Without the knowledge of this art, it is impossible to employ our silk in a profitable manner, for unless the raw material is properly reeled, it cannot be exported abroad nor manufactured at home into those fine stuffs for which we pay so large an annual amount to foreign countries.

Having had the good fortune in finding in Mr. D'Homergue, a person well skilled in that mode of preparing our native silk either for exportation or home manufacture, 1 established last summer under his direction an experimental filature of ten reels, in which twenty women were em-ployed; in consequence of which a market for cocoons was immediately opened at Philadelphia, whither they were brought for sale from almost every part of the United States. The farmers brought them from different parts of this state, and received cash for them, which they had never done before. 'They were brought it is true, in small quantities, but there is no doubt that the culture of silk will be thereby promoted, and that in the course of a few years, if this important object shall receive the national encouragement, which a bill now before Congress gives reason to expect, silk worms will be raised in great plenty all over Pennsylvania. The climate being peculiarly well adapted to the cultivation of the Mulberry tree, which will thrive even in our poorest soil.

The flag which I have the honour to send to you is offered as a visible proof of the facts $I$ have just stated, no silk stuff of the same kind has ever been or ever attempted: in this country, and none can be made without a perfect knowledge of the art of preparing the raw material. I beg you will be so good as to present this flag most respectfully in my name to your honorable House, as a sample. of a new and interesting branch of American manufacture; 
as a token of my high respect; and as a proof of my sincere devotion to the interests of the State of Pennsylvania, which has been from early youth my cherished home, and where I hope with my latest breath, to offer my last fervent prayer for her happiness and prosperity.

I have the honour to be,

With the highest respect,

Sir, your most ob'dt humble serv't.

PE'TER S. DU PONCEAU.

To the Hon. Freberick Smiti, Speaker of the House of Representatives of the Commonwealth of Pennsylvania.

\section{REPORT OF THE COMMIT'TEE.}

That this beautiful specimen of American agriculture and manufactures, carried through all its process in Pennsylvania, is a practical result of the utmost importance to the wealth and prosperity of the state. Its agriculture has long suffered from restriction to certain staples, of which the production has increased, while the foreign demand is greatly diminished. By the valuable experiments of $\mathrm{Mr}$. Du Ponceau and Mr. D'Homergue it is established that the climate, the soil, and the waters of Pennsylvania, are eminently congenial with the culture of silk, which it is believed may be cultivated with advantage throughout the United States. If so, this inestimable product must become a great staple of the country. Adverting to the fact that but forty-six years ago, an American vessel with cotton on board, was seized at Liverpool under the impression that cotton was not the growth of America; and to the fact, that last year more than six hundred and forty thousand bags of American cotton were imported at that port, there is nothing unreasonable in the anticipation that a similar development may attend American silk.

In every country producing silk, it has become the 
fruitful means of industry and wealth. Every manufacturing country incapable of producing, has spared no efforts to naturalize it. Added to the other products of this state and Union, its benefits must be incalculable. It leaves all other employments unimpaired, and affords domestic occupation to females and children, who may rear the cocoons, and reel them into raw silk. Many millions, as Mr. Du Ponceau states, are the tribute money paid for it by the United States to foreign countries.

From the increase of the coasting trade of Philadelphia, which has nearly trebled itself within the last eight years, encouraging and unquestionable proof is afforded, that the agriculture, manufactures, and mineral wealth of the state, are in active and increasing demand. If, as is understood to be the case, vessels loaded with the coal of Pennsylvania are destined to sail for France in the ensuing season, the foreign trade of the state may derive great augmentation from that source. Even the quantity of flour exported from Philadelphia to Europe has much increased of late; and wool bears better prices throughout the country.

With these flattering prospects, no aid within the power of legislation should be withheld from the endeavor to domesticate silk, and unite so important a staple to the others. In France, Italy, Flanders, Spain, and England, no wealth or honours were spared by government, when the object was to cherish and reward the culture of this superior article, which, in all ages and nations, from the earliest era to the present, has been in universal request and of the highest value.

The flag presented by Mr. Du Ponceau is a proof of the natural and artificial resources we possess ; and that in the practical skill of Mr. D'Homergue, the country enjoys the fortunate means of obtaining all the instruction and demonstration necessary for the complete attainment and indefinite extension of the difficult art of reeling silk, without 
which raw silk for manufacturing, or of the merchantable quality saleable in foreign markets cannot be produced.

The patriotic, disinterested, and most praiseworthy exertions of Mr. Du Ponceau to establish the art among us, entitle him to the gratitude of the state. They constitute another of the public services of a citizen whose career, beginning in the army of the revolution, and continued in the department of foreign affairs, afterwards distinguished by eminent accomplishments in jurisprudence and other sciences, has exalted him to become the successor of Jefferson as president of that Philosophical Society which was founded by Franklin. To the duties and distinctions of such a career, Mr. Du Ponceau, in the decline of life, superadds a noble effort to confer upon his country the inestimable advantages of the introduction of silk, and to join that to the many honors of which his venerable years are full.

The committee trust that it will not be deemed inappropriate to display this flag in the hall of the Representatives of Pennsylvania, near the Speaker's chair-the same that was once filled by the President of that immortal Congress, which in the city of Philadelphia, declared the independence of these United States.

They therefore respectfully submit the following resolutions.

Resolved, That the Representatives of the Commonwealth of Pennsylvania accept, with great sensibility and satisfaction, the silk flag of the United States, presented to them by Peter S. Du Poncean, as an auspicious promise of national wealth and prosperity, and a proof of the patriotism of the distinguished donor.

Resolved, That the flag be displayed in a conspicuous part of this House under the direction of the Speaker, and that the Clerk be directed to communicate to Mr. Du Ponceau copies of these resolutions and report. 





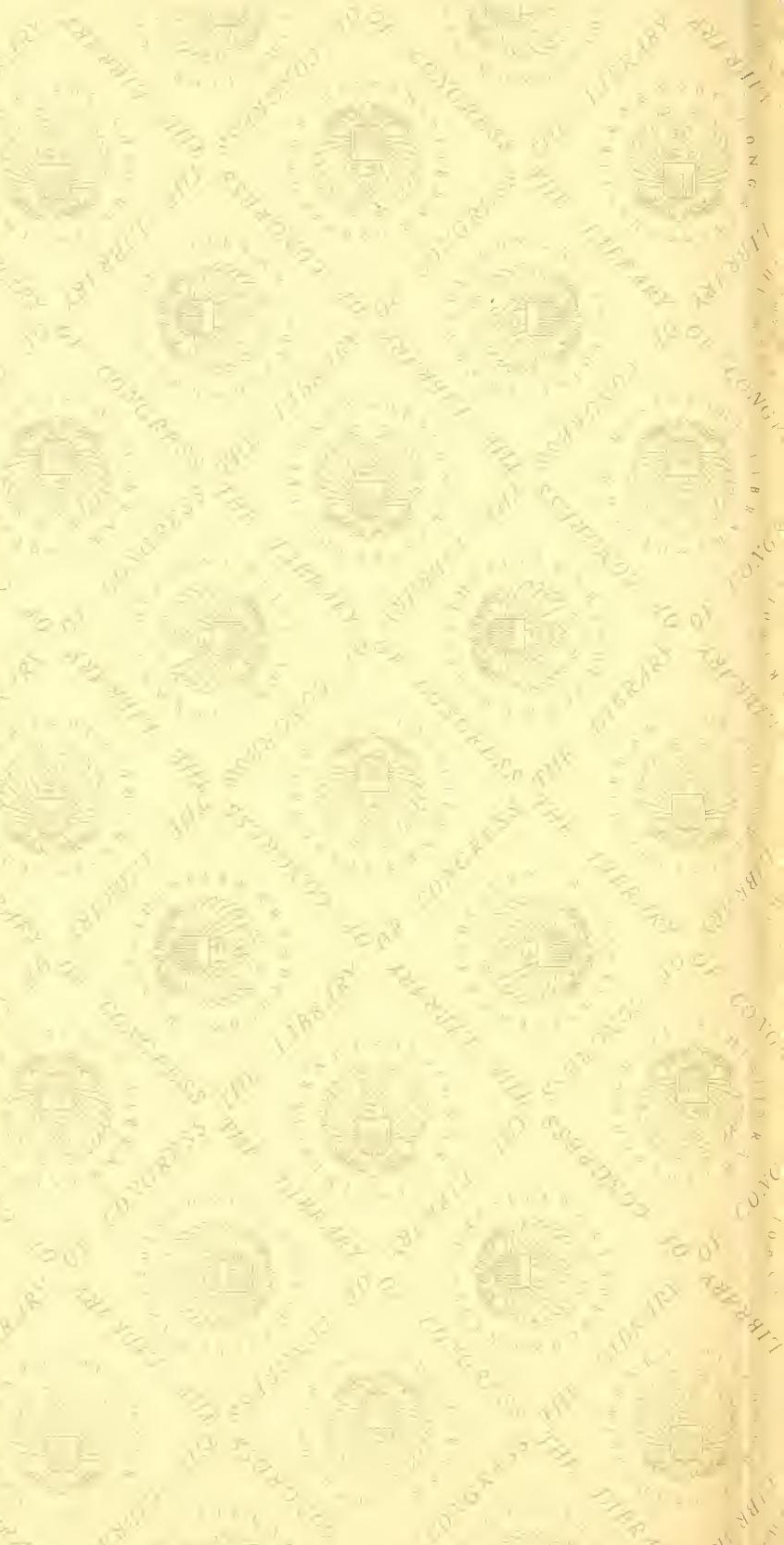


$x=$

$\sqrt{=-}$

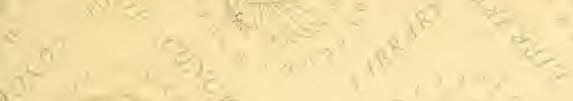

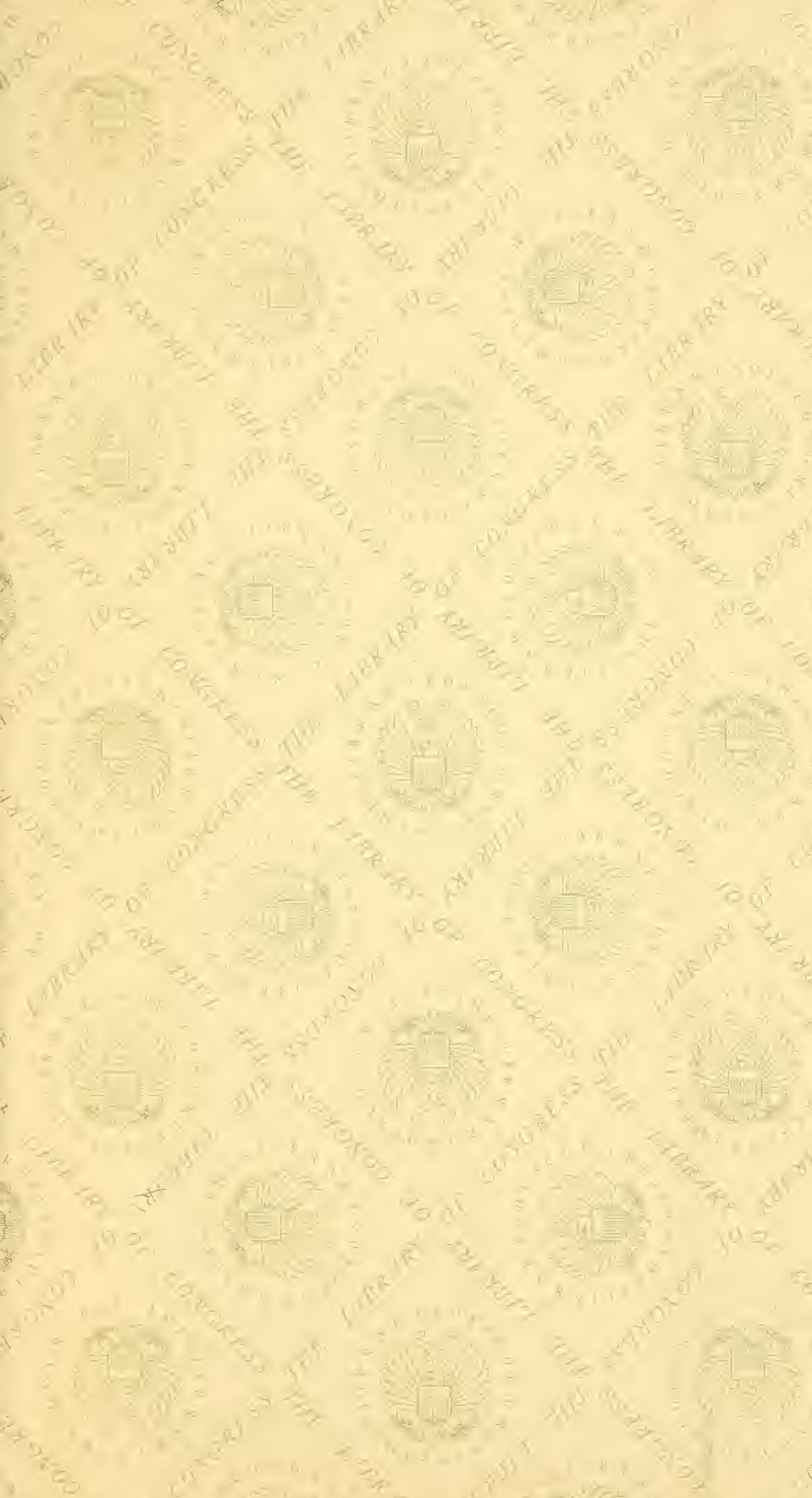




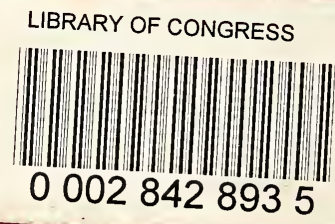

